\title{
Lanthanide Doped Wide Band Gap Semiconductors: Intra- $4 f$ Luminescence and Lattice Location Studies
}

\author{
Dissertation \\ zur Erlangung des Doktorgrades \\ der Mathematisch-Naturwissenschaftlichen Fakultäten \\ der Georg-August-Universität zu Göttingen
}

vorgelegt von

Ulrich Vetter

aus Stuttgart-Bad Canstatt

Göttingen 2003 


\section{D7}

Referent: $\quad$ Prof. Dr. Hans C. Hofsäß

Koreferent: $\quad$ Prof. Dr. Rainer G. Ulbrich

Tag der mündlichen Prüfung: 15. Juli 2003 
To

Olga and Nikita 



\section{Contents}

\begin{tabular}{lll}
\hline Abstract & 1
\end{tabular}

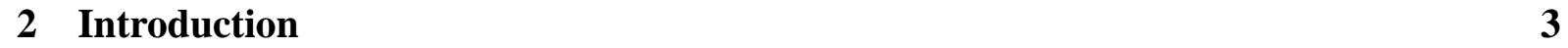

2.1 Lanthanide doped semiconductors . . . . . . . . . . . . . . . 3

2.2 The aim of this work $\ldots \ldots \ldots \ldots \ldots$

\begin{tabular}{|lll}
3 & Fundamentals and characterization techniques & 9
\end{tabular}

3.1 Luminescence properties of triply ionized lanthanides . . . . . . . . . . . . . . 9

$3.1 .1 \quad$ Basic properties . . . . . . . . . . . . . . . 10

3.1 .2 Free ion energy matrix and Dieke diagram . . . . . . . . . . . 12

3.1 .3 The crystal field . . . . . . . . . . . . . . . . 20

3.1 .4 Induced electric dipole and magnetic dipole transitions . . . . . . . . . 24

3.1 .5 Temperature-dependent phenomena: electron phonon interaction . . . . 32

3.1 .6 Relaxation phenomena: time-resolved spectroscopy . . . . . . . . . . . 36

3.1 .7 Numerical details and extensions . . . . . . . . . . . . . . . . . . . . . 39

$3.1 .8 \mathrm{Eu}^{3+}, \mathrm{Tm}^{3+}$ and $\mathrm{Gd}^{3+} \ldots \ldots \ldots \ldots . \ldots . \ldots 41$

3.2 Electron emission channeling . . . . . . . . . . . . . . . . . 45

3.2.1 Solutions to the Schrödinger equation . . . . . . . . . . . . 45

3.2.2 Dechanneling . . . . . . . . . . . . . . 47

3.2 .3 Experimental parameters . . . . . . . . . . . . . . 50

3.2 .4 Numerical details and extensions . . . . . . . . . . . . . . . . 50

3.2.5 Lanthanide isotopes for emission channeling investigation . . . . . . . 51

3.3 Ion implantation . . . . . . . . . . . . . . . . . . . . . . . . 57

3.4 Cathodoluminescence . . . . . . . . . . . . . . . . 57

3.5 Mössbauer spectroscopy $\ldots \ldots \ldots$

3.5.1 Lanthanide isotopes for Mössbauer spectroscopy investigation . . . . . 60

3.5.2 Mössbauer isotopes for thin film investigations . . . . . . . . . . . 62

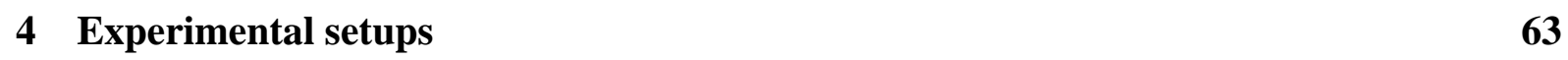

4.1 The luminescence spectrometer. . . . . . . . . . . . . . . . . 63 
$4.1 .1 \quad$ Chamber, vacuum and cooling system . . . . . . . . . . . . . 64

$4.1 .2 \quad$ Spectrograph and collection optics . . . . . . . . . . . . . . . . 64

4.1 .3 Detectors . . . . . . . . . . . . . . . . . . . 66

4.1 .4 Electron gun $\ldots \ldots \ldots \ldots \ldots \ldots$

4.1 .5 Light sources . . . . . . . . . . . . . . . . . . . . 68

4.1 .6 Pulsed cathodoluminescence unit . . . . . . . . . . . . . . . . . 69

4.1 .7 Correction of experimental spectra $\ldots \ldots \ldots \ldots \ldots$. . . . . . . 71

4.2 The emission channeling setup . . . . . . . . . . . . . . . . . . 73

4.3 The Mössbauer spectroscopy setup . . . . . . . . . . . . . . . . . . . 75

4.3 .1 Deetee detector . . . . . . . . . . . . . . . . . 75

4.3 .2 CEMS detector . . . . . . . . . . . . . . . . . . . . . 76

4.4 Other experimental facilities $\ldots \ldots \ldots \ldots$. . . . . . . . . . 77

5 Lanthanide implanted wide band gap III-V nitrides and carbides 79

5.1 Aluminum nitride . . . . . . . . . . . . . . . . . . . . . . . . . . . 79

$5.1 .1 \quad$ Lattice location of ${ }^{169} \mathrm{Yb}$ in $\mathrm{AlN} \ldots \ldots \ldots$. . . . . . . . 80

$5.1 .2 \mathrm{Gd}^{3+}$ doped AlN - an attractive 318 nm UV light emitter . . . . . . . 82

$5.1 .3 \quad \mathrm{Eu}^{3+}$ doped AlN . . . . . . . . . . . . . . . . . 86

$5.1 .4 \mathrm{Tm}^{3+}$ in AlN - blue light emission at room temperature . . . . . . . . 88

5.1 .5 Summary . . . . . . . . . . . . . . . . . . . 92

$5.2 \quad$ Cubic boron nitride $\ldots \ldots \ldots \ldots$. . . . . . . . . . . . . . . 93

$5.2 .1 \quad$ Lattice location of ${ }^{139} \mathrm{Ce}$ in c-BN . . . . . . . . . . . . . . . . . 94

5.2 .2 Luminescence properties of $\mathrm{Eu}^{3+}$ and $\mathrm{Tm}^{3+}$ in c-BN $\ldots \ldots \ldots . .95$

5.2 .3 Summary . . . . . . . . . . . . . . . . . . . . . . 100

5.3 Silicon carbide . . . . . . . . . . . . . . . . . . . . . . . . 102

5.3.1 Lattice location of ${ }^{169} \mathrm{Tm}$ in $3 \mathrm{C}-\mathrm{SiC},{ }^{167} \mathrm{Tm}$ in $4 \mathrm{H}-\mathrm{SiC}$ and ${ }^{169} \mathrm{Yb}$ in

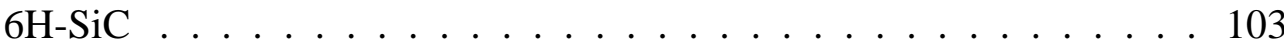

$5.3 .2 \quad \mathrm{Tm}^{3+}$ doped $6 \mathrm{H}-\mathrm{SiC}-$ infrared light emission at $805 \mathrm{~nm} \ldots \ldots$

5.3 .3 Summary . . . . . . . . . . . . . . . . . . . . . 110

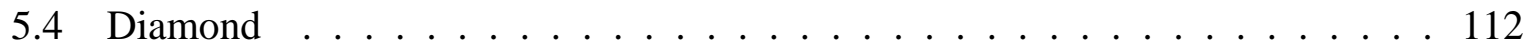

$5.4 .1 \quad$ Lattice location of ${ }^{141} \mathrm{Ce}$ and ${ }^{149} \mathrm{Gd}$ in diamond . . . . . . . . . . . 112

5.4 .2 Luminescence properties of thulium and europium in diamond . . . . . 115 
5.4 .3 Summary $\ldots \ldots \ldots \ldots \ldots \ldots \ldots \ldots \ldots$

6 Thin ta-C and c-BN films doped with Europium during growth 118

6.1 Experimental . . . . . . . . . . . . . . . . . . . . . . . . . . . . 119

6.2 Results . . . . . . . . . . . . . . . . . . . . . . . . . . 120

6.3 Summary . . . . . . . . . . . . . . . . . . . . . . . . . . . . . . 124

\begin{tabular}{|lll}
7 & Crystal field analysis for $\mathbf{T m}^{3+}\left(4 f^{12}\right)$ and $\mathbf{G d}^{3+}\left(4 f^{7}\right)$ in AIN & 125
\end{tabular}

7.1 Experimental CL Stark level spectra of $\mathrm{Tm}^{3+}$ in AlN $\ldots \ldots \ldots$

7.2 Energy level calculations for $\mathrm{Tm}^{3+}$ in AlN $\ldots \ldots \ldots \ldots 131$

7.3 Experimental CL Stark level spectra of $\mathrm{Gd}^{3+}$ in AlN $\ldots \ldots \ldots$

7.4 Energy level calculations for $\mathrm{Gd}^{3+}$ in $\mathrm{AlN} \ldots \ldots \ldots$

7.5 Branching ratios and the $\Omega_{2}$ parameter $\ldots \ldots \ldots \ldots \ldots$

7.6 Phonon side bands . . . . . . . . . . . . . . . . . . . . . . . . . . . . . 144

7.7 Summary and discussion $\ldots \ldots \ldots \ldots \ldots \ldots$

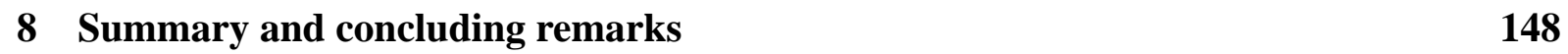

\begin{tabular}{|lr}
\hline A Appendix & 153
\end{tabular}

A.1 Mean free ion parameters for $\mathrm{Eu}^{3+}, \mathrm{Gd}^{3+}$ and $\mathrm{Tm}^{3+} \ldots \ldots \ldots \ldots$. . . . . 153

A.2 Substrates . . . . . . . . . . . . . . . . . . . . . . . . . . . 154

\begin{tabular}{ll}
\hline List of Figures & 157
\end{tabular}

\begin{tabular}{ll}
\hline List of Tables & 161
\end{tabular}

\begin{tabular}{ll}
\hline Bibliography & 163
\end{tabular} 
CONTENTS 


\section{Abstract}

Luminescence properties, along with lattice locations of lanthanide implanted into wide band gap semiconductors, are presented in this work. This study focuses on semiconductors with the widest currently known band gap among their group. These are represented by aluminum nitride and cubic boron nitride for the group of III-V nitride semiconductors, as well as diamond and $6 \mathrm{H}$-silicon carbide for the group of carbide semiconductors. The method of choice for doping at this stage is ion implantation, preferably with low ion fluences, thereby keeping implantation damage as low as possible. This also provides that the lanthanides represent impurities, neither altering the hosts properties nor significantly influencing each other. The focus is on a few selected lanthanides, namely thulium and europium, complemented by the use of gadolinium in one case. In a first attempt these systems are characterized by cathodoluminescence spectroscopy, accompanied by lattice location studies of lanthanides in the same host matrices. In the luminescence investigations those systems are identified which are suitable for a crystal field analysis. The aim of this analysis is to identify the local symmetry of the implanted ions.

An introduction to the field of lanthanide-doped semiconductors is given in chapter 2 , providing an overview of current research trends and characterization techniques. This is accompanied by a critical reflection on the role of radioactive lanthanides and hyperfine measurements in the field of lanthanide-doped semiconductor physics, with respect to the use of radioactive lanthanides in this work.

Chapter 3 provides a condensed presentation of the fundamentals of luminescence of triply ionized lanthanides, the emission channeling method and other characterization techniques used in this work. The main focus is on the calculation of the energy matrix to provide the knowledge required to perform a crystal field analysis and to understand the luminescence spectra of lanthanide-doped semiconductors. The presentation of theory is thereby accompanied by selected references to existing literature. Triply ionized lanthanides are classified according to their energy level structure and transition probabilities, with a focus on $\mathrm{Eu}^{3+}, \mathrm{Tm}^{3+}$, and $\mathrm{Gd}^{3+}$, used as luminescence probes in this work. For other characterization techniques such as emission channeling and Mössbauer spectroscopy an overview of applicable isotopes is given with a description of the experimental energy spectra.

The experimental setups assembled and used in this work are discussed in chapter 4 , mainly the 
luminescence spectrometer for high resolution and time-resolved cathodoluminescence measurements. Their characteristics are summarized and possible error sources are discussed.

In chapter 5 lattice location studies as well as cathodoluminescence investigations of the wide band gap semiconductors $2 \mathrm{H}$-aluminum nitride, cubic boron nitride, $3 \mathrm{C}-, 4 \mathrm{H}-$-, and $6 \mathrm{H}-$ silicon carbide and diamond, implanted with europium, thulium and gadolinium, are presented.

Doping of europium into thin films of cubic boron nitride and tetrahedral amorphous carbon during growth is discussed in chapter 6. Here, mass-selected ion beam deposition is used as an alternative to ion implantation doping.

Chapter 7 is the main part and goal of this work. It presents a detailed crystal field analysis for thulium and gadolinium in aluminum nitride. Based on high resolution cathodoluminescence Stark level spectra the symmetry of the ions is determined. Additionally, further characteristics of these systems with regard to relative intensities of selected intra- $4 f$ electron transitions are deduced.

In chapter 8 , a condensed summary of the results of this work is given, together with concluding remarks. 


\section{Introduction}

\subsection{Lanthanide doped semiconductors}

Only two decades ago it was discovered that doping semiconductors with lanthanides is a promising way to realize light emitters, their luminescence arising from radiative intra- $4 f$ electron transitions of the triply ionized lanthanides doped into these semiconductors [1]. Since then a race has started for these light emitting devices, using various hosts and lanthanides, and based on various motivations. As in the beginning only semiconductors with relatively small optical band gaps were available in sufficient purity and quantities, researchers drew their attention to the implementation of infrared light emitting devices, which are based on the intra- $4 f$ electron transition between the first excited and the ground state of $\operatorname{Er}^{3+}$ (see e.g. [2, 3, 4, 5, 6, 7, 8, 9]). The application was obvious, as the energy of the ${ }^{4} I_{13 / 2} \rightarrow{ }^{4} I_{15 / 2}$ intra- $4 f$ electron transition between the first excited and the ground state of $\mathrm{Er}^{3+}$ matches the most pronounced absorption minimum of $\mathrm{SiO}_{2}$, which is used for optical fibre communication. Thus the commercial prospect seemed to be very attractive. Indeed, up to now, studies on $\mathrm{Er}^{3+}$ doped semiconductors have made up the majority of scientific publications found in the literature (see e.g. Si [10, 11, 12, 13, 14, 8], Ge [15], SiGe [16], GaAs [17], GaN: [18, 19, 20, 21, 22, 23, 24, 25, 26], $\mathrm{SiC}$ [27, 28, 29] ). However, the first implementation of these light emitters, mainly $\mathrm{Er}^{3+}$-doped silicon was rather disappointing because of thermal quenching of the luminescence. Even so, researchers found that the quantum efficiency of these devices was rather large, showing that research in this field had opened up promising new paths. Since the early 90's, new semiconductors with larger band gaps have been sufficiently available to take the next step in the implementation of lanthanide doped light emitters, emitting visible luminescence. The ideal material was discovered in the direct band gap semiconductor gallium nitride $(\mathrm{GaN})$. Lanthanide doped $\mathrm{GaN}$ showed its capability as a light emitter from the infrared to the blue region of the electromagnetic spectrum (e.g. GaN:Pr ${ }^{3+}$ [30, 31], GaN:Sm${ }^{3+}$ [32, 33], $\mathrm{GaN}_{\mathrm{Eu}}{ }^{3+}$ [34, 35], GaN: $\mathrm{Tb}^{3+}$

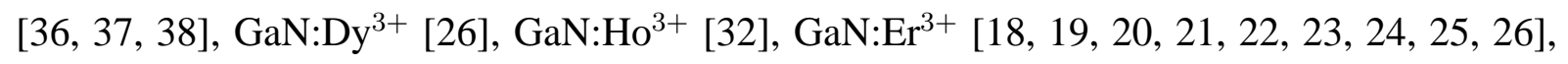
$\left.\mathrm{GaN}: \mathrm{Tm}^{3+}[39,40,26,41,42]\right)$. Additionally it is advantageous over small band gap semiconductors, as it overcomes thermal quenching. Because the band gap of GaN is still rather small with regard to possible energy levels of the triply ionized lanthanides, this substrate is currently supplemented by the very wide band gap semiconductor aluminum nitride (AIN) (see e.g. AlN:Eu ${ }^{3+}$ [43], AlN: $\mathrm{Gd}^{3+}$ [44], AlN: $\mathrm{Tb}^{3+}$ [43], $\mathrm{AlN}: \mathrm{Er}^{3+}$ [45, 46, 47, 48, 49], AlN: $\mathrm{Tm}^{3+}$ [50] ) as well as (cubic) boron nitride $(\mathrm{BN})$ (see e.g. $\mathrm{BN}: \mathrm{Eu}^{3+}$ [51, 52, 53], BN: $\mathrm{Tb}^{3+}$ [54, 51], $\mathrm{BN}: \mathrm{Tm}^{3+}$ [51, 52], ). This extends the application to the implementation of ultraviolet light 
sources, allowing for a possible application as phosphor illuminators, which are important for the implementation of white light emitters [55, 56].

The main goal in using lanthanide implanted semiconductors has been the implementation of highly efficient light emitters [57] and solid state lasers [58, 59]. The discovery of new appropriate host matrices is followed by years of studying the physical properties and mechanisms underlying light emission of the lanthanides in these hosts. This process may last decades just for a certain ion in a certain host, as can be seen from $\mathrm{Si}^{-\mathrm{Er}^{3+}}$. In contrast to investigations of lanthanide doped compounds, which account for most of the experimental work in the last 40 years, the study of lanthanide doped semiconductors is somewhat more complicated. This is mainly due to the excitation processes and the low overall amount of lanthanide ions in the host. Inorganic transparent lanthanide compounds are usually studied with the help of absorption and (site-selective) excitation spectroscopy using polarized or unpolarized laser light. These spectroscopic methods are, with a few exceptions [19], usually not applicable in the case of implanted semiconductors, mainly because of the low concentration and overall amount of the lanthanide ions in the host. Important information such as the symmetry of the lanthanides sites ${ }^{1}$, and a knowledge of the exact positions of the energy levels, which are a typical outcome of absorption and (site-selective) excitation spectroscopy, are mostly hidden from the spectroscopist. Moreover, the excitation mechanism is simply no longer just the absorption of a photon, but involves excitation through the (quasi-)particles in the semiconductor host, e.g. electrons, holes, excitons and phonons. The realization of an efficient light emitter is equivalent to an efficient generation of energy carriers, efficient transfer of energy from these carriers to the lanthanide impurities and efficient radiative electron recombination of the excited impurities. Both the transfer of energy and the de-excitation can be investigated using time-resolved spectroscopy. Options to manipulate these processes are limited. Sometimes energy transfer can be significantly increased by co-implantation of an additional element, in most cases one belonging to group VII (see e.g. Si:Er ${ }^{3+}: \mathrm{F}$ [11], $\mathrm{Si}^{-E^{3+}}{ }^{\mathrm{F}}$ [13, 60], $\mathrm{Si}^{\mathrm{E}} \mathrm{Er}^{3+}: \mathrm{O}$ [13, 12, 6, 60, 61, 62], $\mathrm{Si}: \mathrm{Er}^{3+}: \mathrm{C}$ [61], $\mathrm{Si}: \mathrm{Er}^{3+}: \mathrm{N}$ [61], AlN: $\mathrm{Er}^{3+}: \mathrm{O}$ [39], a-AlN: $\mathrm{Er}^{3+}: \mathrm{H}$ [46, 47], a-SiC: $\mathrm{Er}^{3+}: \mathrm{H}$ [63] ). In some cases, e.g. $\mathrm{Si}^{\mathrm{Er}^{3+}}: \mathrm{O}$, this leads verifiably to the formation of small clusters which act as quantum wells and successfully trap excited carriers.

\section{Excitation}

Throughout the years, different excitation mechanisms of lanthanide impurities in semiconductors have been proposed and experimentally verified. The mechanisms may be divided into

\footnotetext{
${ }^{1}$ The term site is used in different coherences throughout this work. Methods like luminescence spectroscopy and hyperfine interaction methods use the term site for the local environment, which is mainly defined by the nearest ligands. In contrast, while talking about the the emission channeling method, the term site denotes the position of the ions within the unit cell, disregarding the real local environment.
} 


\subsection{LANTHANIDE DOPED SEMICONDUCTORS}

three different categories. The first one involves energy transfer from excitons to the $4 f$ electron system, which is the dominant mechanism in case of photoluminescence, (in some cases) cathodoluminescence and electroluminescence under forward biased conditions [64] The second category, where excitation of the lanthanide center is due to the inelastic scattering of hot electrons at the $4 f$ electron system, is usually found in electroluminescence under reverse biased conditions [64, 9, 65] and (in some cases) cathodoluminescence. Finally, the third one represents direct pumping with photons. The excitation processes involving excitons can be distinguished further:

- Energy transfer from donor-acceptor pairs to a lanthanide center. The free or bound excitons in the host are trapped by the lanthanide center, thereby transferring their energy to the $4 f$ electron system. This process, also known as defect Auger recombination was described by Godlewski et al. [67, 68], who argued that an exciton bound to an ion will break rotational and space symmetry, thus relaxing the transition selection rules. This explains why the excitation cross section for this process is much larger than that for impact excitation and impact ionization, which is typically $10^{-12} \mathrm{~cm}^{2}$ (see e.g. Ref. [69]).

- The lanthanide trap: a lanthanide ion creates a new level in the band gap of the host. This level acts as a trap to bind carriers, either electrons or holes. This charged complex may

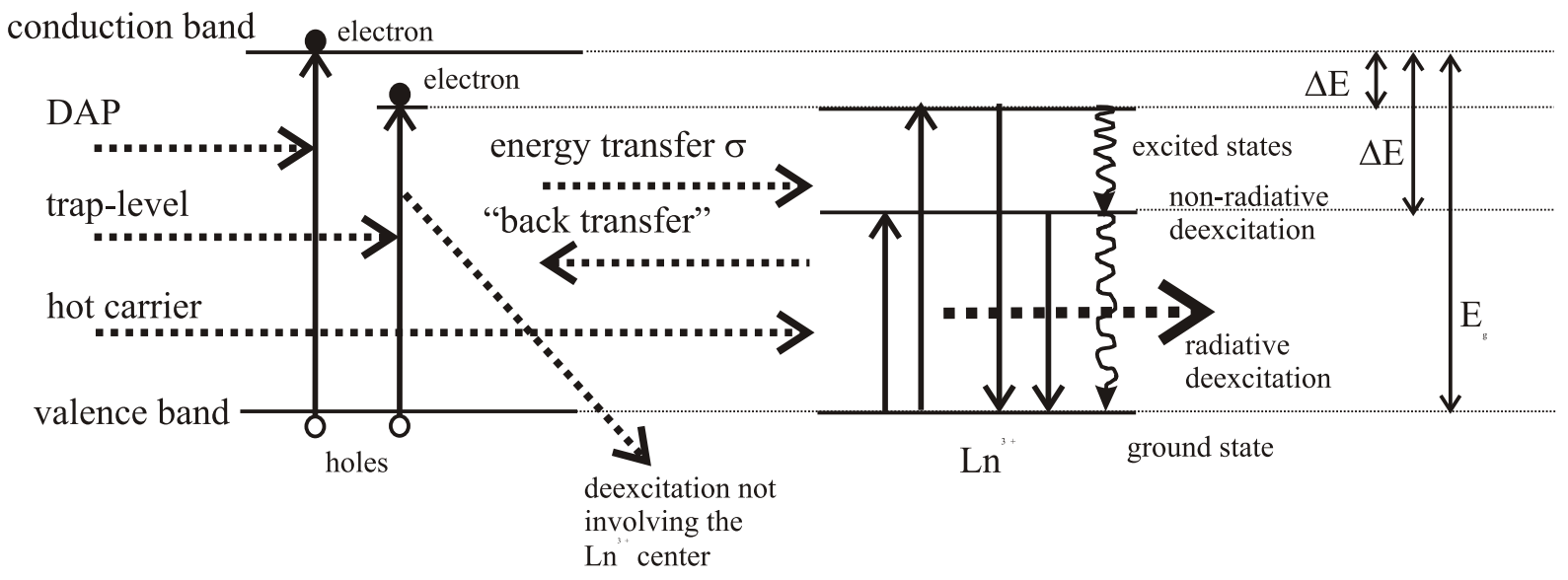

Figure 2.1: $\mathrm{Ln}^{3+}$ excitation mechanisms in semiconductors [66]. Excitation of the $4 f$ electron system is accomplished by energy transfer from donor-acceptor pair excitons, energy transfer involving lanthanide traps or inelastical scattering of hot carriers. Upon excitation of the $4 f$ electron system either a photon is emitted or nonradiative decay takes place, for example in the form of a nonradiative "back-transfer" process or nonradiative (multi-)phonon emission. 
in turn attract a carrier of opposite charge, thereby forming a bound exciton. Transfer of energy from this bound exciton to the $4 f$ electron system leads to excitation, in the same manner as described above for the defect Auger recombination process.

The excitation involving hot carries can also be subdivided into two different cases:

- Impact excitation: a hot carrier inelastically scatters at the $4 f$ electron system, thus transferring energy. This interaction was calculated by Jiaqi et al. [70]. It was shown that the impact excitation cross section is in first order of approximation proportional to the matrix element in the electric dipole operator. As electric dipole transitions within the $4 f$ shell are parity forbidden to first order, this process has a rather low cross section of typically $10^{-18} \mathrm{~cm}^{2}$ to $10^{-20} \mathrm{~cm}^{2}$ (see e.g. Ref. [71]).

- Impact ionization: this process is essentially similar to the impact excitation process but the lanthanide ion is ionized by a hot carrier. Because this process is not parity forbidden, a larger cross section of typically $10^{-16} \mathrm{~cm}^{2}$ or more is expected [72], but the process may only take place if the ionization energy is smaller than the band gap of the host [72].

The third process, i.e. direct excitation e.g. with laser light, is seldom applicable to the case of lanthanide doped semiconductors, as it requires a large number of lanthanide ions to be incorporated into the host matrix due to the low cross section of the spontaneous absorption process.

Similar to the transfer of energy to the $4 f$-electron system, the reverse process may take place, leading to nonradiative deexciation. While in ideal inorganic solids nonradiative deexcitation is expected to occur mainly through the emission of phonons or transfer of energy between lanthanides in case of larger concentrations [73], possible de-excitation mechanisms in semiconductors again additionally involve excitons or the lanthanide trap. This makes the formulation of the de-excitation mechanisms much more difficult.

\section{Characterizing lanthanide doped semiconductors}

The characterization of lanthanide-doped semiconductors refers to the determination of the electronic, magnetic, vibrational and optical influence and properties of the introduced impurities. Optical properties cover the intra-atomic transition of the impurities but also the luminescence due to induced defects. In the case of an optical band gap of the semiconducting material, the most attractive optical characterisation method is the use of polarized absorption spectroscopy, which can be applied to transparent hosts containing a sufficient number of ions. If the number of ions is below the detection limit of these two methods - a situation typically found in ion 


\subsection{LANTHANIDE DOPED SEMICONDUCTORS}

implanted semiconductors - other methods such as photoluminescence (PL), cathodoluminescence (CL) and electroluminescence (EL) are useful. While PL as well as CL are usually used for excitation purposes only, without exploring the nature of the excitation, EL is not only the realization of light emitting devices, but also directly allows to determine excitation cross sections [70, 3, 9]. Questions regarding the local symmetry of the ions may be answered by means of luminescence spectra, provided that a sufficient number of transitions is detected $\left(\mathrm{GaN}: \mathrm{Pr}^{3+}[31]\right.$, $\mathrm{GaN}: \mathrm{Sm}^{3+}$ [33], GaN:Tb ${ }^{3+}$ [38]). If this direct optical method is not applicable, the symmetry may be obtained from electron paramagnetic resonance (EPR) spectroscopy ( $\mathrm{Si}^{2}{ }^{3+}$ [74, 75] and $\mathrm{SiC}: \mathrm{Er}^{3+}$ [76, 28]) or Zeemann spectrocopy ( $\mathrm{Si}^{2} \mathrm{Er}^{3+}$ [77]). The electrical properties of lanthanides in the semiconductor host are usually explored using (radiotracer) deep level transient spectroscopy (DLTS) (see e.g. Si:Er ${ }^{3+}[78,74,79,80,81,82]$ ) and $\mathrm{SiC}^{2} \mathrm{Er}^{3+}$ [83]).

\section{The role of hyperfine interactions and radioactive impurities}

Besides the characterization of lanthanides in semiconductors by means of the characterization techniques mentioned above, the application of hyperfine methods such as Mössbauer spectroscopy [84] or perturbed $\gamma \gamma$-angular correlation spectroscopy [85] (PAC) can provide useful information [86, 87]. A typical result of Mössbauer spectroscopy is the determination of the charge state of the implanted ions (see e.g. Si:Eu [88, 89, 90]). Because the triply ionized lanthanides $\mathrm{Ce}^{3+}$ through $\mathrm{Yb}^{3+}$ are paramagnetic ions, the electric field gradient $V$ at the nucleus is temperature dependent on the thermal population of the ground state of the ions,

$$
V=V_{C F}+V_{4 f}(T)
$$

where $V_{C F}$ is the temperature independent contribution of the crystal field at the nucleus and $V_{4 f}(T)$ is the contribution by the $4 f$ electrons [91]. Performing temperature dependent hyperfine measurements will yield the ground state splitting. This will provide the valence of the ions, and also point to the symmetry of the crystal field. Although this method was applied successfully to a number of hosts with both Mössbauer spectroscopy [92, 93] and PAC [94], up to now no such hyperfine investigations of lanthanides in semiconductors seem to exist. This fact appears to be mainly due to the insufficient availability of radioactive lanthanide ions. Their production for implantation purposes usually requires on-line isotope separators. It can also be related to a stagnating detector development for these methods in recent years. 


\subsection{The aim of this work}

This work presents studies of lanthanide doped wide band gap semiconductors with the aim to investigate general optical properties and furthermore to determine both the position of the implanted ions in the unit cell as well as the local symmetry. The study focuses on group III-V nitride semiconductors AlN and c-BN, and the carbides diamond and SiC. Selected lanthanides are chosen as luminescence probes. Experiments to identify lattice sites for lanthanides in $\mathrm{AlN}$, c-BN, diamond as well as $3 \mathrm{C}-, 4 \mathrm{H}-$, and $6 \mathrm{H}-\mathrm{SiC}$ were performed, focusing on new radioactive probe isotopes. Luminescence studies applying cathodoluminescence, investigated the spectra for thulium and europium implanted $\mathrm{AlN}, \mathrm{c}-\mathrm{BN}$, diamond and $6 \mathrm{H}-\mathrm{SiC}$. They were complemented by investigations for gadolinium in AlN. The most significant intra- $4 f$ electron transitions related to europium, thulium, and gadolinium, are furthermore characterized by time-resolved cathodoluminescence spectroscopy. Based on the outcome, those systems were identified for which a crystal field analysis based on luminescence spectra is possible. The crystal field analysis was performed in order to identify the symmetry of the ions in the semiconductor host matrix as well as dominating transitions in the luminescence spectra. Additionally, intensities of these dominant transitions will be discussed. 


\section{Fundamentals and characterization techniques}

\subsection{Luminescence properties of triply ionized lanthanides}

Long before the implementation of lanthanide-doped semiconductors as light emitters, "intra-4f luminescence" attracted researcher's attention. This luminescence, which arises from radiative electron transitions within the $4 f$-shell of the paramagentic, triply ionized lanthanides $\mathrm{Ce}^{3+}$ through $\mathrm{Yb}^{3+}$, has remarkable features. One of these is simply its occurrence, first reported by J. Becquerel in 1907 [95]. Later it was discovered that the nature of this luminescence, due to radiative electron transitions with the $4 \mathrm{f}-$ shell [96, 97], is of electric dipole character. It is therefore in contrast to the prediction of electrodynamics, which demands the change of parity between initial and final state of the electron systems in the case of electric dipole radiation and a spherically symmetric potential. This demand is known as Laporte's rule. At first, no explanation for this mystery was found. Then, in the early 1960's, two researchers, B.R. Judd [98] and S. Ofelt [99], independently discovered the solution: the admixture of states of opposite parity through a crystal field, thus allowing radiative electric dipole transitions within the 4fshell. Since the invention of this theory of induced electric dipole radiation, enormous efforts have been made to develop a powerful theory based on numerous spectroscopic data [100]. This theory allows the calculation of energy levels and transition probabilities. This development has been continuously progressing for about forty years now, mainly because the luminescence properties of triply ionized lanthanides could always contribute to current research demands. Despite their interesting magnetic properties (for an introduction see e.g. the book of Jensen and Mackintosh [101]), the application of lanthanides in the field of light emitters, including the field of solid state laser physics [102], plays a very important role.

The most interesting feature of lanthanide spectra are their very sharp luminescent lines much resembling the spectra found in the case of free ions. This is due to the fact that the $4 \mathrm{f}$ electrons are effectively shielded from surrounding crystal fields because of the outer filled $5 \mathrm{~s}$ and $5 \mathrm{p}$ shells. This phenomenon is also known for the actinides and, to a lesser extent, for transition metals. Because of this shielding the energetic positions of the energy levels of the $4 \mathrm{f}$ electron systems are almost unaffected, and the linewidths are often only slightly broadened. This characteristic allows systematic investigations of the crystal field in transparent materials [103].

Much effort has been made to understand and compute the spectra of lanthanides since the invention of the luminescence theory by Judd and Ofelt in the sixties. Because the theory is very voluminous, only the approach and the final solution will be sketched in this work. The 
theoretical treatment of energy level and intensity calculations are based on group theory. An excellent summary is given in a book of Judd [104]. Several larger compendia exist giving an overview of historical developments [105, 106], experimental data [100] and crystal field calculations [103]. In the following chapters, a condensed summary of the theory will be given, which is necessary to understand the calculation of free ion energy levels and the crystal field as well as transition intensities. Unless otherwise mentioned, the description follows the one given by Görller-Walrand and Binnemans [106, 107].

\subsubsection{Basic properties}

Neutral lanthanides possess the electron configuration $4 f^{N} 5 s^{2} 5 p^{6} s^{2}$, except for gadolinium, which shows the configuration $4 f^{N} 5 s^{2} 5 p^{6} 5 d^{1} s^{2}$ [108], where the number of $4 f$ electrons $N$ ranges from 2 for cerium to 14 for ytterbium. The dominating oxidation state is $3+$, although $2+$ is frequently found for some compounds and $4+$ is known for cerium as well. This is in contrast to the actinide series where oxidation numbers up to +8 are known. The fact that the oxidation state in some cases tends to $2+$ can be understood by looking at the ionization energy needed for the removal of another $4 f$ electron: it is highest for europium and ytterbium $(\sim 2400 \mathrm{~kJ} / \mathrm{mol})$ and lowest for cerium and gadolinium $(<2000 \mathrm{~kJ} / \mathrm{mol})$, which explains why europium often tends to the oxidation state $2+$. In the triply ionized state the electron configuration becomes $4 f^{N} 5 s^{2} 5 p^{6}$, where the number of $4 f$ electrons ranges from 1 for $\mathrm{Ce}^{3+}$ to 13 for $\mathrm{Yb}^{3+}$. This is visualized in table 3.1. The filling of the $4 f$ shell can be explained by Hund's rules, which predict that the term with the highest quantum number $S$ has the lowest energy and if there are several terms with the same $S$, the one with the highest angular momentum quantum number $L$ has the lowest energy. Furthermore, because of spin-orbit coupling, the terms ${ }^{2 S+1} L$ are split into levels $J=L+S, L+S-1, \ldots,|L-S|$, where for less than half-filled shells, the term with the smallest $J$ lies lowest in energy. Otherwise the term with the largest $J$ has the lowest energy. In this manner one can easily predict the ground state of the ions, as shown in table 3.1. as well as the number of possible ${ }^{2 S+1} L$ and possible ${ }^{2 S+1} L_{J}$ configurations for each of the ions.

An interesting property of the lanthanide series is, with a few exceptions, the decrease in both the ionic and the covalent radii and the increase in Pauling's electronegativity with increasing nuclear charge $Z$. This can be attributed to the poor shielding abilities of the $4 f$ electrons thus leading to a diminution of the radial integrals of the outer $5 s$ and $5 p$ electrons with increasing nuclear charge $Z$. This effect is the main reason for differences found in compounds throughout the lanthanide series, e.g. a slightly different chemistry, but it may also have an influence on 
3.1. LUMINESCENCE PROPERTIES OF TRIPLY IONIZED LANTHANIDES

\begin{tabular}{cc|cccccccc|c|c|c|c}
\hline \hline & & $\mathrm{m}_{l}$ & -3 & -2 & -1 & 0 & 1 & 2 & 3 & $S^{\mathrm{a}}$ & $L^{\mathrm{b}}$ & $J^{\mathrm{c}}$ & ${ }^{2 S+1} L_{J} \mathrm{~d}$ \\
\hline $\mathrm{Ce}^{3+}$ & $4 \mathrm{f}^{1}$ & & $\downarrow$ & & & & & & & $1 / 2$ & 3 & $5 / 2$ & ${ }^{2} F_{5 / 2}$ \\
$\mathrm{Pr}^{3+}$ & $4 \mathrm{f}^{2}$ & & $\downarrow$ & $\downarrow$ & & & & & & 1 & 5 & 4 & ${ }^{3} H_{4}$ \\
$\mathrm{Nd}^{3+}$ & $4 \mathrm{f}^{3}$ & & $\downarrow$ & $\downarrow$ & $\downarrow$ & & & & & $3 / 2$ & 6 & $9 / 2$ & ${ }^{4} I_{9 / 2}$ \\
$\mathrm{Pm}^{3+}$ & $4 \mathrm{f}^{4}$ & & $\downarrow$ & $\downarrow$ & $\downarrow$ & $\downarrow$ & & & & 2 & 6 & 4 & ${ }^{5} I_{4}$ \\
$\mathrm{Sm}^{3+}$ & $4 \mathrm{f}^{5}$ & & $\downarrow$ & $\downarrow$ & $\downarrow$ & $\downarrow$ & $\downarrow$ & & & $5 / 2$ & 5 & $5 / 2$ & ${ }^{6} H_{5 / 2}$ \\
$\mathrm{Eu}^{3+}$ & $4 \mathrm{f}^{6}$ & & $\downarrow$ & $\downarrow$ & $\downarrow$ & $\downarrow$ & $\downarrow$ & $\downarrow$ & & 3 & 3 & 0 & ${ }^{7} F_{0}$ \\
$\mathrm{Gd}^{3+}$ & $4 \mathrm{f}^{7}$ & $\downarrow$ & $\downarrow$ & $\downarrow$ & $\downarrow$ & $\downarrow$ & $\downarrow$ & $\downarrow$ & $7 / 2$ & 0 & $7 / 2$ & ${ }^{8} S_{7 / 2}$ \\
$\mathrm{~Tb}^{3+}$ & $4 \mathrm{f}^{8}$ & $\downarrow \uparrow$ & $\downarrow$ & $\downarrow$ & $\downarrow$ & $\downarrow$ & $\downarrow$ & $\downarrow$ & 3 & 3 & 6 & ${ }^{7} F_{6}$ \\
$\mathrm{Dy}^{3+}$ & $4 \mathrm{f}^{9}$ & & $\downarrow \uparrow$ & $\downarrow \uparrow$ & $\downarrow$ & $\downarrow$ & $\downarrow$ & $\downarrow$ & $\downarrow$ & $5 / 2$ & 5 & $15 / 2$ & ${ }^{6} H_{15 / 2}$ \\
$\mathrm{Ho}^{3+}$ & $4 \mathrm{f}^{10}$ & & $\downarrow \uparrow$ & $\downarrow \uparrow$ & $\downarrow \uparrow$ & $\downarrow$ & $\downarrow$ & $\downarrow$ & $\downarrow$ & 2 & 6 & 8 & ${ }^{5} I_{8}$ \\
$\mathrm{Er}^{3+}$ & $4 \mathrm{f}^{11}$ & & $\downarrow \uparrow$ & $\downarrow \uparrow$ & $\downarrow \uparrow$ & $\downarrow \uparrow$ & $\downarrow$ & $\downarrow$ & $\downarrow$ & $3 / 2$ & 6 & $15 / 2$ & ${ }^{4} I_{15 / 2}$ \\
$\mathrm{Tm}^{3+}$ & $4 \mathrm{f}^{12}$ & & $\downarrow \uparrow$ & $\downarrow \uparrow$ & $\downarrow \uparrow$ & $\downarrow \uparrow$ & $\downarrow \uparrow$ & $\downarrow$ & $\downarrow$ & 1 & 5 & 6 & ${ }^{3} H_{6}$ \\
$\mathrm{Yb}^{3+}$ & $4 \mathrm{f}^{13}$ & & $\downarrow \uparrow$ & $\downarrow \uparrow$ & $\downarrow \uparrow$ & $\downarrow \uparrow$ & $\downarrow \uparrow$ & $\downarrow \uparrow$ & $\downarrow$ & $1 / 2$ & 3 & $7 / 2$ & ${ }^{2} F_{7 / 2}$ \\
\hline \hline
\end{tabular}

a $S=\left|\sum m_{s}\right|$ with $\mathrm{m}_{s}=+1 / 2(\uparrow)$ or $\mathrm{m}_{s}=-1 / 2(\downarrow)$.

${ }^{\mathrm{b}} L=\left|\sum m_{l}\right|$

c $J=|L-S|$ (less than half-filled shell), $J=L+S$ (otherwise)

d Predicted ground state according to Hund's rules.

Table 3.1: Filling of the $4 f$ shells according to Hund's rules.

the symmetry in ion implanted systems. This has recently been demonstrated by Gruber et al. [31, 33, 38] who showed that the symmetry of $\mathrm{Tb}^{3+}$ implanted into $\mathrm{GaN}$ is different from that found for $\mathrm{Pr}^{3+}$ and $\mathrm{Sm}^{3+}$. This decrease in symmetry was attributed to the differing ionic radii.

Another general property of the triply ionized lanthanides is the large number of possible coordination numbers found in compounds, leading to a larger number of possible symmetries [106]. Coordination numbers up to 8 and 9 are frequently found, but higher coordination numbers often exist as well. This is in contrast to the limited number of coordination numbers found for transition metals. The reason for this can be seen in the more ionic character of the bonding between lanthanides and surrounding ligands, in contrast to an emphatic covalent character of the bonding in the case of the transition metals. It is interesting to look at lanthanide implanted semiconductors from this point of view, because the typical coordination number encountered is 4 , and therefore out of character for typical lanthanide compounds. 


\subsubsection{Free ion energy matrix and Dieke diagram}

The treatment of triply ionized lanthanides in solids is handled according to the solution of the Schrödinger equation. To find the correct Hamiltonian that determines the energy levels one has to take into account the kinetic energy $H_{K E}$ and the potential energy $H_{P E}$ of the $4 \mathrm{f}$-electrons, which leads to the following Hamiltonian:

$$
H_{0}=H_{K E}+H_{P E}=-\frac{\hbar^{2}}{2 m} \sum_{i=1}^{N} \Delta_{i}-\sum_{i=1}^{N} \frac{Z^{*} e^{2}}{r_{i}}
$$

where the sum runs over all $4 \mathrm{f}$ electrons. $Z^{*}$ represents the atomic potentials screened by the inner electrons. Both the term representing the kinetic energy of the electrons and the one representing the potential energy are spherically symmetric. Solving the Schrödinger equation at this point leads to the approximate solutions $\left|n l m_{l} m_{s}\right\rangle$

$$
\psi_{n l m_{l} m_{s}}(r, \theta, \phi)=\frac{1}{r} R_{n l}(r) Y_{l m_{l}}(\theta, \phi) \chi_{m_{s}},
$$

characterized by the quantum numbers $n, l, m_{l}$ and $m_{s}$. This is often referred to as the mean field approximation. At this point the energy levels of the $4 f^{N}$ configuration (characterized by the quantum numbers $n l$, where $n=4$ and $l=3$ for $4 \mathrm{f}$ electrons) are highly degenerate. The level of degeneracy is $\left(\begin{array}{c}14 \\ N\end{array}\right)$. In case of $\mathrm{Tm}^{3+}\left(4 f^{12}\right), \mathrm{Eu}^{3+}\left(4 f^{6}\right)$ and $\mathrm{Gd}^{3+}\left(4 f^{7}\right)$ which will be discussed within this work, the degeneracy is 91-fold, 3003-fold and 3432-fold, respectively. The set of wavefunctions 3.2 is the starting point for the application of perturbation theory, because the energy eigenvalues of the Hamiltonian $H_{0}$ belonging to equation 3.2 are much larger than the contributions which will be discussed below, in the sense that

$$
H_{0} \gg H_{i}
$$

where $H_{i}$ stands for all other perturbations. In fact, the energy eigenvalues of the Hamiltonian (equation 3.1) can be estimated from the known binding energies of the $4 f$ electrons, which are in the range of more than $1000 \mathrm{eV}$. The perturbations are responsible for (partial) removal of the degeneracy of the levels encountered in equation 3.2. The most important perturbations are: 


\subsection{LUMINESCENCE PROPERTIES OF TRIPLY IONIZED LANTHANIDES}

- Coulomb interaction

- Spin-orbit interaction

- Two-particle interactions

- Three-particle interactions

Although more interactions can be taken into account, the named ones are mostly sufficient to reproduce the energy level scheme of the triply ionized lanthanides. The interactions are handled writing down and solving a general $4 f$ matrix element for each interaction separately with the intention of parametrization.

\subsubsection{Coulomb interaction}

The next contribution to be taken into account for the Hamiltonian is the Coulomb interaction $H_{E L}$ between $4 f$ electrons. Similar to the treatment of the crystal field, which will be discussed later, the electrostatic interaction $H_{E L}$ is expanded into Legendre polynomials and the sphericalharmonic addition theorem is applied, thereby expressing $H_{E l}$ in tensor-operator form [109]:

$$
\begin{aligned}
H_{E L} & =-e^{2} \sum_{i} \sum_{j, j>i} \frac{1}{\left|r_{j}-r_{i}\right|} \\
& =-e^{2} \sum_{i} \sum_{j, j>i} \sum_{k=0}^{\infty} \frac{r_{<}^{k}}{r_{>}^{k+1}} P_{k}\left(\cos \left(r_{i}, r_{j}\right)\right) \\
& =-e^{2} \sum_{i} \sum_{j, j>i} \sum_{k=0}^{\infty} \frac{r_{<}^{k}}{r_{>}^{k+1}} \sum_{q=-k}^{k} \frac{4 \pi}{2 k+1}(-1)^{q} Y_{k}^{q}\left(\theta_{i}, \phi_{i}\right) Y_{k}^{-q}\left(\theta_{j}, \phi_{j}\right) \\
& \equiv-e^{2} \sum_{i} \sum_{j, j>i} \sum_{q=-k}^{k} \frac{r_{<}^{k}}{r_{>}^{k+1}}(-1)^{q} C_{-q}^{(k)}(i) C_{q}^{(k)}(j) \\
& \equiv-e^{2} \sum_{i} \sum_{j, j>i} \frac{r_{<}^{k}}{r_{>}^{k+1}} C_{i}^{(k)} C_{j}^{(k)}
\end{aligned}
$$

with the $C_{q}^{(k)}$ defined as

$$
C_{q}^{(k)}(i):=\sqrt{\frac{4 \pi}{2 k+1}} Y_{k}^{q}\left(\theta_{i}, \phi_{i}\right)
$$


Therefore, the general matrix element that has to be calculated is of the type

$$
\left(f^{N} \gamma S L M J_{M}\left|C_{i}^{(k)} \cdot C_{j}^{(k)}\right| f^{N} \gamma^{\prime} S^{\prime} L^{\prime} M^{\prime} J_{M}^{\prime}\right) .
$$

Rewriting equation 3.4 results in a more convenient form [104]:

$$
\begin{aligned}
H_{E L} & =-e^{2} \sum_{i} \sum_{j, j>i} \frac{r_{<}^{k}}{r_{>}^{k+1}}\left(u_{i}^{(k)} u_{j}^{(k)}\right)\left\langle f\left\|C^{(k)}\right\| f\right\rangle \\
& \equiv \sum_{k=0,2,4,6} f_{k} F^{k}
\end{aligned}
$$

where $f_{k}$ are the operators and $F^{k}$ are the well-known Slater integrals or parameters

$$
F^{k} \equiv e^{2} \int_{0}^{\infty} \int_{0}^{\infty} \frac{r_{<}^{k}}{r_{>}^{k+1}} R^{2}\left(r_{i}\right) R^{2}\left(r_{j}\right) d r_{i} d r_{j} .
$$

It can be shown that the parameter $F^{0}$ does shift the whole $4 f$ configuration, but it has no influence on the relative energetic positions of the $4 f^{N}$ configurations. This parameter is therefore excluded and, along with shifting contributions of other interactions, summed up in the parameter $E_{A V E}$ (see equation 3.12). The fact that the sum in equation 3.6 runs over even values of $k$, is a constraint found in the evaluation of the $f^{(k)}$ operators.

\subsubsection{Spin-orbit interaction}

The spin-orbit interaction describes the interaction between the spin of the electron and its magnetic moment. The Hamiltonian of the spin-orbit interaction $H_{S O}$ is obtained from the nonrelativistic form of the Dirac equation and takes a well-known form in the case of a spherically symmetric electrostatic potential [109]:

$$
H_{S O}=\zeta \sum_{i} \mathbf{s}_{i} \cdot \mathbf{l}_{i}
$$

where the sum runs over all $4 f$ electrons. Equation 3.8 can be rewritten in a form similar to equation 3.6 [104]: 


$$
H_{S O}=-e^{2} \sum_{i}\left(u_{i}^{(1)} u_{j}^{(1)}\right)\langle 4 f|| \mathbf{s} \| 4 f\rangle\langle 4 f \| \mathbf{l}|| 4 f\rangle
$$

According to Judd [104], the matrix element $\sum_{i} \mathbf{s}_{i} \cdot \mathbf{l}_{i}$ is evaluated for $4 f$ electrons in the following way:1

$$
\begin{aligned}
& \left(4 f^{N} \gamma S L J M_{J}\left|\sum_{i=1} \mathbf{s}_{i} \cdot \mathbf{l}_{i}\right| 4 f^{N} \gamma^{\prime} S^{\prime} L^{\prime} J^{\prime} M_{J}^{\prime}\right) \\
& =12 \sqrt{14} \delta\left(J, J^{\prime}\right) \delta\left(M_{J}, M_{J}^{\prime}\right)(-1)^{S^{\prime}+L+J} \\
& \times\left\{\begin{array}{ccc}
S & S^{\prime} & 1 \\
L^{\prime} & L & J
\end{array}\right\}[S]\left[S^{\prime}\right][L]\left[L^{\prime}\right]^{\frac{1}{2}}(-1)^{\frac{7}{2}+S+L} \\
& \times \sum_{\bar{\theta}}\left(\theta \{ | \overline { \theta } ) \theta ^ { \prime } \left\{\mid \bar{\theta}(-1)^{\bar{S}+\bar{L}}\left\{\begin{array}{ccc}
S & 1 & S^{\prime} \\
\frac{1}{2} & \bar{S} & \frac{1}{2}
\end{array}\right\}\left\{\begin{array}{ccc}
L & 1 & L^{\prime} \\
3 & \bar{L} & 3
\end{array}\right\}\right.\right.
\end{aligned}
$$

where $\theta$ represent the coefficients of fractional parentage. The spin-orbit interaction is parametrized by only one parameter, $\zeta$. For the free ion $\zeta$ ranges from $747 \mathrm{~cm}^{-1}$ for $\mathrm{Ce}^{3+}$ to $2644 \mathrm{~cm}^{-1}$ for $\mathrm{Yb}^{3+}$ [106]. Upon including spin-orbit interaction, the free ion energy levels can no longer be characterized by the quantum numbers $S$ and $L$, because $H_{S O}$ does not commute with $S$ and $L$ separately but with the sum $J=L+S$. This is illustrated in figure 3.1 where, upon including $H_{S O I}$, the labeling of states changes from ${ }^{2 S+1} L$ to ${ }^{2 S+1} L_{J}$.

\subsubsection{Other interactions}

A correction to the electrostatic two-particle Coulomb interaction, namely the linear configuration interaction $H_{L C}$, has been given by Rajnak and Wybourne [110]. This interaction is parameterized using the three parameters $\alpha, \beta$, and $\gamma$ in the form of

$$
H_{L C}=\alpha L(L+1)+\beta G\left(G_{2}\right)+\gamma G\left(R_{7}\right)
$$

where $L$ is the total angular momentum operator, and $G\left(G_{2}\right)$ and $G\left(R_{7}\right)$ are the Casimir's operators for the groups $G_{2}$ and $G_{7}$. Table A.1 shows typical values for the parameters $\alpha, \beta$,

\footnotetext{
${ }^{1}$ From here on the abbreviation $2 X+1=[X]$ is used.
} 
and $\gamma$.

In case of more than two or less than twelve $4 f$ electrons, in addition to the configuration interaction shown above, non-linear three-particle configuration interactions must be taken into account. The parameterization of this interaction was demonstrated by Judd [111] who obtained the following Hamiltonian:

$$
H_{N L C}=t_{2} T^{2}+t_{3} T^{3}+t_{4} T^{4}+t_{6} T^{6}+t_{7} T^{7}+t_{8} T^{8}
$$

where $t_{i}$ are the operators and $T^{i}$ are the parameters. Values of the $T^{i}$ parameters for the case of $\mathrm{Eu}^{3+}\left(4 f^{6}\right)$ and $\mathrm{Gd}^{3+}\left(4 f^{7}\right)$ are listed in table A.1. The magnetically correlated spin-spin and spin-other-orbit interaction of the $4 f$ electrons was first treated by Judd et al [112] and parameterized in the form:

$$
H_{S S, S O O}=\sum_{k=0,2,4} m_{k} M^{k}
$$

where $M^{k}$ are the Marvin integrals [113] treated as adjustable parameters, and $m_{k}$ are the operators. Finally, the electrostatic correlated spin-orbit interactions were parameterized by Goldschmidt et al. [114] and Judd et al. [112]:

$$
H_{E C S O}=\sum_{k=2,4,6} p_{k} P^{k}
$$

where $p_{k}$ are the operators and $P^{k}$ the adjustable parameters.

In figure 3.1, the magnitude of $H_{L C}, H_{S S, S O O}$ and $H_{E C S O}$ is shown with regard to energy shift of the $\mathrm{Tm}^{3+}$ levels if only Coulomb and spin-orbit interaction are taken into account. Obviously, the energetic position is affected for only a few levels, mainly for ${ }^{1} S_{0}$ and ${ }^{1} I_{6}$, and, to a smaller extent, for the ${ }^{3} P_{0,1,2}$ and ${ }^{1} D_{2}$ levels. 


\subsection{LUMINESCENCE PROPERTIES OF TRIPLY IONIZED LANTHANIDES}

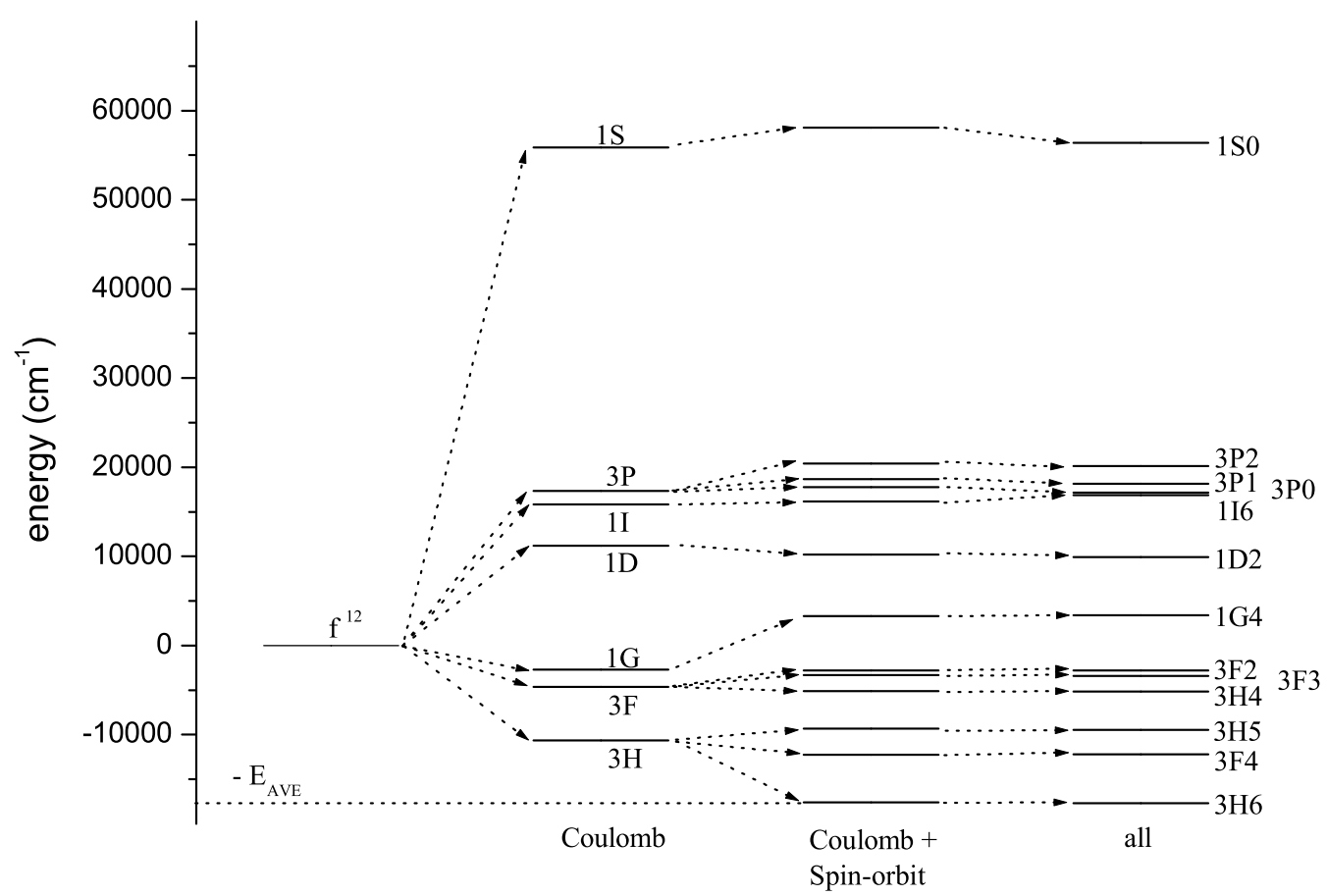

Figure 3.1: Calculated $\mathrm{Tm}^{3+}$ free ion energy level diagram using the mean free ion parameters listed in table A.1. The energy of the levels is given relative to the unperturbed configuration. The diagrams show the energetic positions for Coulomb interaction, Coulomb and spin-orbit interaction and all interactions.

\subsubsection{The final Hamiltonian}

Taking all mentioned interactions of the free ion into account, the final free ion Hamiltonian is written as follows:

$$
\begin{aligned}
H & =H_{0}+E_{A V E}+\sum_{k=2,4,6} f_{k} F^{k}+\zeta \sum_{i=1}^{N} \mathbf{s}_{i} \cdot \mathbf{l}_{i}+\alpha L^{2}+\beta G\left(G_{2}\right)+\gamma G\left(R_{7}\right) \\
& +\sum_{i=2,3,4,6,7,8} t_{i} T^{i}+\sum_{k=0,2,4} m_{k} M^{k}+\sum_{k=2,4,6} p_{k} P^{k}
\end{aligned}
$$

For lanthanides with only one $4 f$ electron $\left(\mathrm{Ce}^{3+}, \mathrm{Yb}^{3+}\right)$ this results in just one fitting parameter ${ }^{2}$ $\zeta$. In the case of two $4 f$ electrons $\left(\mathrm{Pr}^{3+}, \mathrm{Tm}^{3+}\right)$, the Coulomb-interaction needs to be accounted for, thus resulting in four parameters. For the remaining ions the interaction is parameterized with 19 or 15 (if only $M^{0}$ and $P^{2}$ are varied) free ion parameters. Although the number of

\footnotetext{
${ }^{2}$ It is not necessary to include $E_{A V E}$ in the fitting process, it can rather be treated as an adjustable parameter.
} 
free ion parameters can be relatively large, it should be mentioned that especially in photoor cathodoluminescence spectroscopy of lanthanide doped semiconductors, where only a few $L S J$ multiplets can be identified, almost all but the Slater- and spin-orbit parameters are kept at fixed values. To decide whether this is reasonable one has to look at the dependence of the energetic positions of the multiplets on each of the parameters. A typical example is the ${ }^{1} S_{0}$ of $\mathrm{Pr}^{3+}$ and $\mathrm{Tm}^{3+}$ which largely determines the parameter $\gamma$. If these levels cannot be detected, $\gamma$ has to be kept at a fixed value.

To compute the energy diagram for each lanthanide the matrix elements for each of the mentioned interactions are calculated. The energy matrix is then diagonalized and solved, using the program package as described in chapter 3.1.7 Even though the energy matrix is hermitian, and therefore only half of it has to be calculated, this is in some cases a very time-consuming task. The corresponding wavefunctions are calculated by applying the coefficients of fractional parentage ${ }^{3}$ As shown in the last chapters for some of the interactions, each interaction is not only parameterizable but also can be calculated easily if the reduced matrix elements under consideration are known.

\subsubsection{The Dieke diagram}

Figure 3.2 shows the energy level structure for lanthanides $\mathrm{Ce}^{3+}$ through $\mathrm{Yb}^{3+}$, known as Dieke diagrams [119]. The $L S J$ levels are calculated using the mean free ion parameters taken from Ref. [106], the level diagrams are cut at $52,000 \mathrm{~cm}^{-1}$. Some of the calculations yield a ground state energy different from zero, which has been corrected for by adjusting the mean free ion parameter $E_{A V E}$. Only for $\mathrm{Ce}^{3+}$ and $\mathrm{Pr}^{3+}$ all the levels are shown. For some ions level energies up to $200,000 \mathrm{~cm}^{-1}$ are possible. The notation of the levels in figure 3.2 is in accordance with the main eigenvector components if spin-orbit coupling is switched off. For some ions, whose levels are very closely spaced, only the most important levels have been assigned, namely those which are expected to take part in luminescent transitions because they are sufficiently spaced from the nearest lower-lying level. The band gap values for some selected semiconductors are shown on the right. The energetic positions of the top of the valence bands have been set to zero in this diagram.

\footnotetext{
${ }^{3}$ A description of how this can be done and the labeling of the states using irreducible representations can be found in the papers of Racah [115, 116, 117, 118]
} 

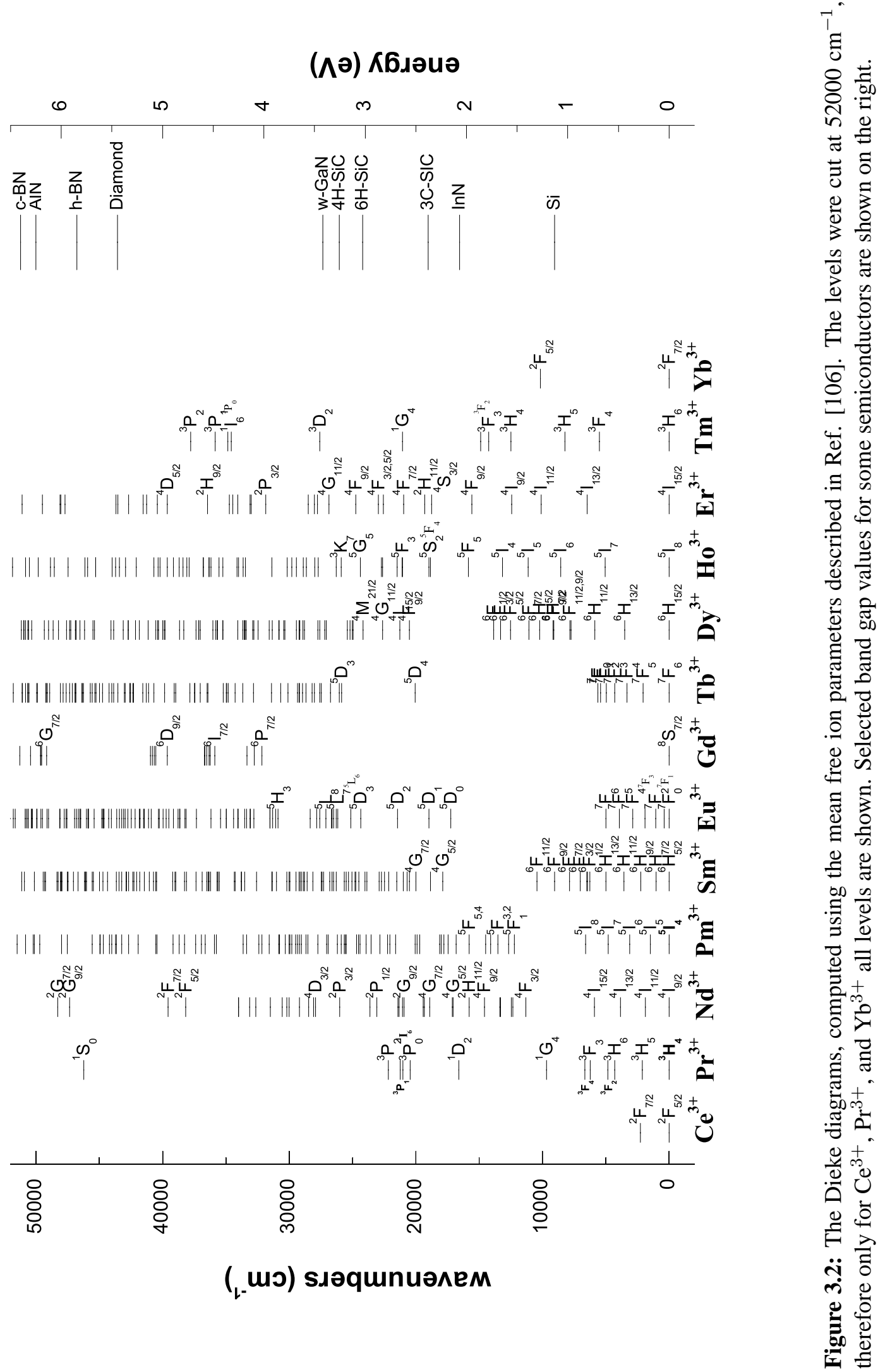


\subsubsection{The crystal field}

So far, each contribution to the Hamiltonian has considerd the ion as free. For a trivalent lanthanide ion inserted into a crystal matrix, another contribution to the Hamiltonian, namely the interaction of the $4 f$ electrons with the surrounding crystal field, has to be taken into account. This interaction is in many ways the most difficult one to treat. Usually, for an ionic compound the point charge model can be applied, in which the surrounding ions are treated as point charges rather than charge distributions. Of course, for many compounds and also for some of the systems investigated in this work, this assumption is oversimplified. Important contributions to the crystal field additionally arise from ligand polarizations, covalent and overlap contributions, and others. A survey of different models which describe more or less covalent systems was given by Garcia and Faucher [120]. In addition, a very useful overview of the calculation of the crystal field- $4 f$ electron interaction is given in the Crystal Field Handbook [103]. In the following, the basic principles of evaluation the crystal field- $4 f$ electron interaction will be shown, based on the point charge model.

A charge distribution $\rho$ at distance $R$ will cause the following potential $V$ at position $r_{i}$ of the $\mathrm{i}^{\text {th }} 4 \mathrm{f}$ electron:

$$
V=\int \frac{\rho(R)}{\left|R-r_{i}\right|} d \tau .
$$

Instead of solving the integral above, it is more descriptive to sum over discrete point charges. Then the potential arising from all point charges $j$ within the lattice at the position of the $4 \mathrm{f}$ electron can be summed up and augmented as follows [106]:

$$
\begin{aligned}
V\left(\mathbf{r}_{\mathbf{i}}\right) & =-e \sum_{j} \frac{Z_{j}}{\left|R_{j}-r_{i}\right|} d \tau \\
& =-e \sum_{j} Z_{j} \sum_{k=0}^{\infty} \frac{r_{<}^{k}}{r_{>}^{k+1}} P_{k}\left(\cos \left(R, r_{i}\right)\right) \\
& =-e \sum_{j} Z_{j} \sum_{k=0}^{\infty} \frac{r_{<}^{k}}{r_{>}^{k+1}} \sum_{q=-k}^{k} \frac{4 \pi}{2 k+1}(-1)^{q} Y_{k}^{q}\left(\theta_{i}, \phi_{i}\right) Y_{k}^{-q}(\theta, \phi)
\end{aligned}
$$




$$
\equiv-e \sum_{j} Z_{j} \sum_{k=0}^{\infty} \sum_{q=-k}^{k} B_{q}^{(k)} C_{q}^{(k)}(i)
$$

with $C_{q}^{(k)}$ and $B_{q}^{(k)}$ defined as

$$
\begin{aligned}
C_{q}^{(k)}(i) & =\sqrt{\frac{4 \pi}{2 k+1}} Y_{k}^{q}\left(\theta_{i}, \phi_{i}\right) \\
B_{q}^{(k)} & =-e \sum_{k=0}^{\infty} Z_{j}(-1)^{q} C_{k}^{-q}(\theta, \phi) \frac{r_{<}^{k}}{r_{>}^{k+1}} .
\end{aligned}
$$

In this case $\left(\left|R_{j}-r_{i}\right|\right)^{-1}$ has been expanded into Legendre polynomials, where $r_{<}$is the smaller and $r_{>}$the larger distance of $\left(R, r_{i}\right)$, and the angle $\omega$ between $R$ and $r_{i}$ is expressed in the polar angles $\theta$ and $\phi$, applying the so called spherical harmonic addition theorem. To return to a charge distribution instead of using discrete point charges in the above equations, the sum over $\mathrm{k}$ has to be replaced by an integral

$$
\sum_{k=0}^{\infty} \longrightarrow \int \ldots . . d \tau
$$

Upon looking at the $B_{q}^{(k)}$, it becomes clear that these do not act on the $4 f$ wavefunctions, but the $C_{q}^{(k)}(i)$ do, as single $4 f$ electron tensor operators. The $B_{q}^{(k)}$ parameters can therefore be brought outside the crystal field matrix element, which is then written and calculated as follows:

$$
\begin{aligned}
& \left\langle 4 f^{N} \gamma S L J M\left|\sum_{i} C_{q}^{(k)}(i)\right| 4 f^{N} \gamma^{\prime} S^{\prime} L^{\prime} J^{\prime} M^{\prime}\right\rangle \\
& =\left\langle 4 f^{N} \gamma S L J M\left|U_{q}^{(k)}\right| 4 f^{N} \gamma^{\prime} S^{\prime} L^{\prime} J^{\prime} M^{\prime}\right\rangle\left\langle 3|| C^{(k)}|| 3\right\rangle \\
& =\sqrt{49}(-1)^{2 J-M+S+k+l}\left[[J]\left[J^{\prime}\right]\right]^{1 / 2} \\
& \times\left(\begin{array}{ccc}
3 & k & 3 \\
0 & 0 & 0
\end{array}\right)\left(\begin{array}{ccc}
J & k & J^{\prime} \\
-M & q & M^{\prime}
\end{array}\right)\left\{\begin{array}{ccc}
J & J^{\prime} & k \\
L^{\prime} & L & S
\end{array}\right\} \\
& \quad \times\left\langle 4 f^{N} \gamma S L|| U_{q}^{(k)}|| 4 f^{N} \gamma^{\prime} S^{\prime} L^{\prime}\right\rangle .
\end{aligned}
$$


The second $3 \mathrm{j}$-symbol is nonzero only if $-M+q+M^{\prime}=0$. This is a general selection rule for transitions between different crystal field levels. Furthermore, the triangle conditions of the $3 j$-symbols requires $k \leq J+J^{\prime}$. The first $3 j$-symbol is nonzero only if $k+6$ is even, therefore $\mathrm{k}$ has to be even as well. The triangle condition furthermore requires that $k \leq 6$. Therefore only those $C_{q}^{(k)}$ with an even value of $k$ are responsible for a splitting of the crystal field levels, and only those with $0 \leq k \leq 6$ have to be considered. Only the even part of the crystal field potential is responsible for a splitting of a ${ }^{2 S+1} L_{J}$ level. As shown by Judd and Ofelt, the odd part of the crystal field potential is responsible for the intensities of induced electric dipole transitions between different multiplets.

By separating the spherical harmonics into a real and an imaginary part, and by using the relation $\exp (i \phi)=\cos (\phi)+i \sin (\phi)$, the potential can be rewritten as (see ref. [106])

$$
\begin{aligned}
V\left(r_{i}\right)=\sum_{k=0}^{7}\left[B_{0}^{(k)} C_{0}^{(k)}(i)\right. & +\sum_{q=1}^{k}\left(B_{q}^{(k)}\left(C_{-q}^{(k)}(i)+(-1)^{q} C_{k}^{(q)}(i)\right)\right. \\
& \left.\left.+{B^{\prime}}_{q}^{(k)} i\left(C_{-q}^{(k)}(i)-(-1)^{q} C_{k}^{(q)}(i)\right)\right)\right]
\end{aligned}
$$

with the $B_{q}^{(k)}$ redefined as follows:

$$
\begin{aligned}
B_{0}^{(k)} & =\sqrt{\frac{4 \pi}{2 k+1}} Y_{k}^{0} \sum_{j}(-Z e)_{j} \frac{r^{k}}{R_{j}^{k+1}} \\
B_{q}^{(k)} & =\sqrt{\frac{4 \pi}{2 k+1}}(-1)^{q} \operatorname{Re} Y_{k}^{q} \sum_{j}(-Z e)_{j} \frac{r^{k}}{R_{j}^{k+1}} \\
{B^{\prime}}_{q}^{(k)} & =\sqrt{\frac{4 \pi}{2 k+1}}(-1)^{q} \operatorname{Im} Y_{k}^{q} \sum_{j}(-Z e)_{j} \frac{r^{k}}{R_{j}^{k+1}}
\end{aligned}
$$

The $B_{q}^{(k)}$ parameters are further restricted by the fact that the crystal field Hamiltonian has to be invariant under all symmetry operations of the point group under consideration. In other words: only those $B_{q}^{(k)}$ for a given symmetry will be non-zero whose $Y_{k}^{(q)}$ will contain the symmetry elements of the point group. Taking the symmetry $\mathrm{C}_{3 v}$ as an example (see figure 3.3), the potential at a $4 f$ electron turns into [106]:

$V_{C_{3 v}}^{\text {even }}=B_{0}^{(2)} C_{0}^{(2)}+B_{0}^{(4)} C_{0}^{(4)}+B_{3}^{(4)}\left(C_{3}^{(4)} C_{-3}^{(4)}\right)+B_{0}^{(6)} C_{0}^{(6)}+B_{3}^{(6)}\left(C_{3}^{(6)} C_{-3}^{(6)}\right)+B_{6}^{(6)}\left(C_{6}^{(6)} C_{-6}^{(6)}\right)$. 
where only the even part of the crystal field is shown, which is responsible for the crystal field splitting.

For many symmetry cases it can be shown that the $B_{q}^{(k)}$ are related to the $B_{0}^{(k)}$. This relation, as well as the signs, are important constraints for the fitting procedure, as it will result in a lower number of parameters as well as a limitation of the fitting range. A typical example with symmetry $\mathrm{T}_{d}$ can be found for $\mathrm{Si}: \mathrm{Er}^{3+}[61,121]$.

Although it is clear that a calculation of the $B_{q}^{(k)}$ parameters on the basis of the point charge model will not result in reliable values, except for highly ionic compounds, equation 3.15 can often be applied, provided that ef fective point charges can be calculated. Görller-Walrand and Binnemans [106] noted that the point charge model does, however, predict the signs of the crystal field parameters correctly.

It can be shown that an evaluation of the energy matrix, including the crystal field, shows nondiagonal matrix elements with respect to the quantum number $M$. Therefore, an alternative
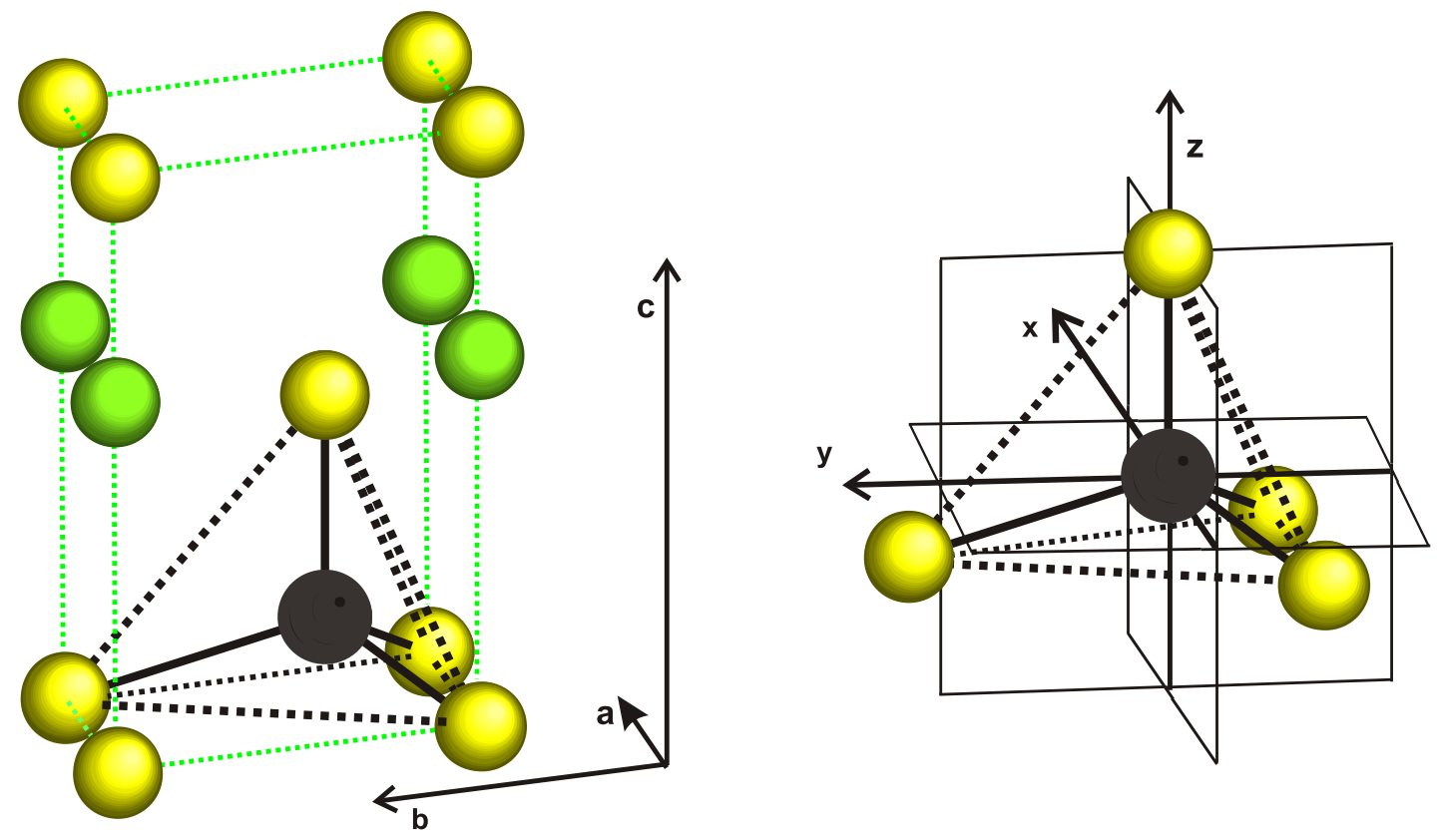

Figure 3.3: Example of symmetry $C_{3 v}$ : shown is the w-AlN unit cell where a lanthanide atom (black) replaces an aluminum atom (green). The nitrogen ligands (yellow) are located at distances $1.50 \AA$ (upper ligand in the graph) and $1.80 \AA$ (other three ligands). The axis of highest symmetry $\left(C_{3}\right)$ is chosen as $\mathrm{z}-\mathrm{axis}$ and corresponds to the $\mathrm{c}-$ axis in the w-AlN unit cell. The radii of the spheres are chosen arbitrary. 
way for labeling the crystal field states has to be found. The most general one is the use of irreducible representations as suggested by Racah. These are noted as $\Gamma_{i}$ (according to Bethe ). Alternatively one may use the Mulliken symbols such as $A, B, E$ and $T$, a correlation between both sets of symbols is listed in Ref. [106]. The advantage of using irreducible representations is that selection rules can be derived, which additionally account for polarization, and can therefore be experimentally verified in polarized absorption spectroscopy for many point groups. As all spectra in this thesis are unpolarized, nothing is gained by using irreducible representations. An alternative way is the classification used by Hellwege who introduced the crystal quantum number $\mu$ :

$$
M=\mu(\bmod q)
$$

It can be shown that the above equation is sufficient to distinguish between the nondiagonal matrix elements appearing in the evaluation of equation 3.14 which are identified by the triangular condition $-M+q+M^{\prime}=0$. The procedures to determine the appropriate crystal field quantum numbers $\mu$ for a certain point group are explained in the literature (see e.g. Refs. [105, 106]). Appropriate values for $\mathrm{C}_{3 v}$ are $\mu=0, \mu=1$ for an even number of $4 f$ electrons (e.g. $\mathrm{Eu}^{3+}$ and $\mathrm{Tm}^{3+}$ ) and $\mu= \pm \frac{1}{2}, \mu= \pm \frac{3}{2}$ for an odd number of $4 f$ electrons (e.g. $\mathrm{Gd}^{3+}$ ). A way to determine $\mu$ by evaluating the energy matrix will be discussed in chapter 3.1 .7 .

\subsubsection{Induced electric dipole and magnetic dipole transitions}

A long time ago it was discovered that transitions observed in lanthanide spectra, which were attributed to electron transitions within the $4 f$ atomic shell, are essentially of electric dipole nature. This was somehow surprising because electric dipole transitions within the $4 f$ shell are parity-forbidden. This can be understood by looking at the electric multipole tensor operator $\mathbf{m}_{\rho}$ of rank $l$ defined as [107]

$$
\mathbf{m}_{\rho}^{(l)}=-e \mathbf{D}_{\rho}^{(l)}=-e \sum_{j} \mathbf{r}_{\rho}^{(l)}\left(r_{j}, \theta_{j}, \phi_{j}\right)
$$

where the sum runs over all $4 f$ electrons. Here $l$ accounts for the multipolarity of the radiation, $l=1$ for dipole radiation, $l=2$ for quadrupole radiation, etc. $\mathbf{r}_{\rho}$ is the position operator of 


\subsection{LUMINESCENCE PROPERTIES OF TRIPLY IONIZED LANTHANIDES}

electron $j$ and $\mathbf{D}_{\rho}^{(l)}$ is the electric dipole transition operator. Both the parity operator $\pi_{\rho}$ and the position operator $\mathbf{r}_{\rho}$ do not commute [122]

$$
\left\langle i\left|\mathbf{r}_{\rho}\right| f\right\rangle=\left\langle i\left|\pi_{\rho}^{+} \pi_{\rho} \mathbf{r}_{\rho} \pi_{\rho}^{+} \pi_{\rho}\right| f\right\rangle=\epsilon_{i} \epsilon_{f}\left(-\left\langle i\left|\mathbf{r}_{\rho}\right| f\right\rangle\right)
$$

This condition is only fulfilled if the initial state $\langle i|$ and the final state $|f\rangle$ have opposite parities. It was independently shown by Judd [98] and Ofelt [99] in the 1960's that the occurrence of electric dipole transitions can be explained by the admixture of states of opposite parity by the crystal field. The following is a very concise version of the description given in ref. [107].

In order to derive the expectation of the electric dipole operator, in a first approximation Judd used a description of an arbitrary initial level $\langle A|$ such that

$$
\langle A| \equiv \sum_{M}\left\langle l^{N} \gamma M\right| a_{M}
$$

where $\gamma$ stands for all quantum numbers needed to uniquely define the state, and $a_{M}$ are expansion coefficients. The same is done for the final level $\left|A^{\prime}\right\rangle$. In first-order perturbation theory, the initial level $\langle A|$ becomes the level $\langle B|$

$$
\langle B|=\sum_{M}\left\langle 4 f^{N} \gamma M\right|+\sum_{K}\left\langle 4 f^{N-1}\left(n^{\prime} l^{\prime}\right) \gamma^{\prime \prime} M^{\prime \prime}\right| b\left(n^{\prime} l^{\prime} \gamma^{\prime \prime} M^{\prime \prime}\right)
$$

where

$$
b\left(n^{\prime} l^{\prime} \gamma^{\prime} M^{\prime}\right)=\frac{\sum_{M} a_{M}\left\langle 4 f^{N} \gamma M\left|\mathbf{V}_{\text {odd }}\right| 4 f^{N-1}\left(n^{\prime} l^{\prime}\right) \gamma^{\prime \prime} M^{\prime \prime}\right\rangle}{E(\gamma)-E\left(n^{\prime} l^{\prime} \gamma^{\prime \prime}\right)}
$$

and where $n^{\prime} l^{\prime}$ belong to configurations other than the $4 f$ shell. From the above equations it can be seen that no admixture of other states appears if the odd part of the potential, $\mathbf{V}_{\text {odd }}$, is zero. This is exactly the case for symmetries with an inversion center, such as $\mathrm{C}_{2 i}$, and others. 
The evaluation of the electric dipole transition operator may then be written as:

$$
\begin{aligned}
& \left\langle B\left|\mathbf{D}_{\rho}^{(1)}\right| B^{\prime}\right\rangle \\
& =\sum_{K, M, M^{\prime}, k, q} a_{M} a_{M^{\prime}} A_{k q} \frac{\left\langle 4 f^{N} \gamma M\left|\mathbf{D}_{\rho}^{(1)}\right| 4 f^{N} \gamma^{\prime} M^{\prime}\right\rangle\left\langle 4 f^{N-1}\left(n^{\prime} l^{\prime}\right) \gamma^{\prime \prime} M^{\prime \prime}\left|\mathbf{D}_{\rho}^{(k)}\right| 4 f^{N} \gamma^{\prime} M^{\prime}\right\rangle}{E\left(\gamma^{\prime}\right)-E\left(n^{\prime} l^{\prime} \gamma^{\prime \prime}\right)} \\
& +\sum_{K, M, M^{\prime}, k, q} a_{M} a_{M^{\prime}} A_{k q} \frac{\left\langle 4 f^{N} \gamma M\left|\mathbf{D}_{\rho}^{(k)}\right| 4 f^{N} \gamma^{\prime \prime} M^{\prime \prime}\right\rangle\left\langle 4 f^{N-1}\left(n^{\prime} l^{\prime}\right) \gamma^{\prime \prime} M^{\prime \prime}\left|\mathbf{D}_{\rho}^{(k)}\right| 4 f^{N} \gamma^{\prime} M^{\prime}\right\rangle}{E(\gamma)-E\left(n^{\prime} l^{\prime} \gamma^{\prime \prime}\right)}
\end{aligned}
$$

where $A_{k q}$ are crystal field parameters 4 .

Judd's final expression for a single transition between two individual Stark levels is:

$$
\begin{aligned}
& \left\langle B\left|\mathbf{D}_{\rho}^{(l)}\right| B^{\prime}\right\rangle \\
& =\sum_{k, q} \sum_{\lambda=\text { even }}[\lambda](-1)^{q+\rho} A_{k q}\left(\begin{array}{ccc}
1 & \lambda & k \\
\rho & -q-\rho & q
\end{array}\right)\left\langle A\left|U_{q+\rho}^{(\lambda)}\right| A^{\prime}\right\rangle \Xi(k, \lambda)
\end{aligned}
$$

with

$$
\begin{aligned}
\Xi(k, \lambda) & =14 \sum_{n^{\prime} l^{\prime}}\left[l^{\prime}\right](-1)^{3+l^{\prime}} \\
& \times\left\{\begin{array}{ccc}
1 & \lambda & k \\
3 & l^{\prime} & 3
\end{array}\right\}\left(\begin{array}{lll}
3 & 1 & l^{\prime} \\
0 & 0 & 0
\end{array}\right)\left(\begin{array}{lll}
l^{\prime} & k & 3 \\
0 & 0 & 0
\end{array}\right) \\
& \times \frac{\left\langle 4 f|\mathbf{r}| n^{\prime} l^{\prime}\right\rangle\left\langle 4 f\left|\mathbf{r}^{(k)}\right| n^{\prime} l^{\prime}\right\rangle}{\Delta\left(n^{\prime} l^{\prime}\right)}
\end{aligned}
$$

where $\langle A|$ are the wavefunctions given in equation 3.23 and $A_{k q}$ are the crystal field parameters. In the same manner, magnetic dipole transitions can be derived, which are parity-allowed within the $4 f$ shell and additionally may even occur under inversion symmetry. The evaluation is similar to the one given above for induced magnetic dipole transitions and is derived by applying the magnetic dipole operator $\mu_{\rho}^{(1)}$ which is defined as

$$
\mu_{\rho}^{(1)}=-\frac{e \hbar}{2 m_{e} c}\left(\mathbf{L}+g_{e} \mathbf{S}\right)_{\rho}^{(1)}
$$

\footnotetext{
${ }^{4}$ These historical crystal field parameters were not explicitly introduced in chapter 3.1.3. Transformation of $B_{q}^{(k)}$ into $A_{k q}$ and vice versa, and other details related to the $A_{k q}$ parameters, are found in the literature [105, 106, 107, 103].
} 


\subsection{LUMINESCENCE PROPERTIES OF TRIPLY IONIZED LANTHANIDES}

where $\mathbf{S}$ and $\mathbf{L}$ are the total spin and total orbital angular momentum operators, respectively; $g_{e}$ is the electron g-factor (2.00223). The corresponding matrix element can be worked out as follows:

$$
\begin{aligned}
& \left(4 f^{N} \gamma S L J M_{J}\left|\left(\mathbf{L}+g_{e} \mathbf{S}\right)^{(1)}\right| 4 f^{N} \gamma^{\prime} S^{\prime} L^{\prime} J^{\prime} M_{J}^{\prime}\right) \\
& =(-1)^{J-M}\left(\begin{array}{ccc}
J & 1 & J^{\prime} \\
-M & \rho & M^{\prime}
\end{array}\right)\left\langle 4 f^{N} \gamma S L J\left\|\left(\mathbf{L}+g_{e} \mathbf{S}\right)^{(1)}\right\| 4 f^{N} \gamma^{\prime} S^{\prime} L^{\prime} J^{\prime}\right\rangle .
\end{aligned}
$$

The reduced matrix element in equation 3.27 can be split into independent terms of $\mathbf{L}$ and $\mathbf{S}$, shown for $\mathbf{L}$ in the following

$$
\begin{aligned}
& \left\langle 4 f^{N} \gamma S L J M_{J}\|\mathbf{L}\| 4 f^{N} \gamma^{\prime} S^{\prime} L^{\prime} J^{\prime} M_{J}^{\prime}\right\rangle \\
& =\delta_{\tau \tau^{\prime}} \delta_{S S^{\prime}} \delta_{L L^{\prime}}(-1)^{S+L+J+1}\left(\begin{array}{ccc}
J & J & S \\
J^{\prime} & L & 1
\end{array}\right)\left[[L][J]\left[J^{\prime}\right] L(L+1)\right]^{\frac{1}{2}} .
\end{aligned}
$$

To obtain the term for $\mathbf{S}, L$ in the equation above has to be replaced by $S$.

For the case of $J=J^{\prime}$ in equation 3.28 , the reduced matrix element in equation 3.27 becomes

$$
\left\langle J||(\mathbf{L}+2 \mathbf{S})^{(1)} \| J\right\rangle=g \sqrt{[J(J+1)[J]]}
$$

where $g$ is the Landé factor. In contrast to induced electric dipole radiation, magnetic dipole transitions are allowed within the $4 f$ shell, although they are usually one order of magnitude smaller than induced electric dipole transitions. Nevertheless, in some cases magnetic dipole transitions can play an important role, e.g. when induced electric dipole transitions are forbidden because of selection rules or under inversion symmetry.

From the final expressions for intensities of induced electric dipole and magnetic dipole transitions selection rules can be derived, which result mainly from the properties of the $3 j-$ and $6 j$-symbols. An overview is given in Table 3.2 . These selection rules are only valid as long as the corresponding quantum numbers are good quantum numbers. The selection rules on $M$ and $S$ break down in the intermediate coupling scheme, the selection rule on $J$ breaks down in the case of $J$-mixing. 


\begin{tabular}{c|c|c}
\hline & ED & MD \\
\hline$\Delta J$ & $|\Delta J| \leq 6$ & $\Delta J=0, \pm 1$ \\
& $|\Delta J|=2,4,6$ if $J=0$ or $J^{\prime}=0$ & $0 \leftrightarrow 0$ forbidden \\
$\Delta M$ & $-(q+\rho)$ & $-\rho(\rho= \pm 1)$ \\
$\Delta L$ & $|\Delta L| \leq 6$ & 0 \\
$\Delta S$ & 0 & 0 \\
$\Delta \tau$ & 0 & 0 \\
$\Delta l$ & \pm 1 & - \\
\hline
\end{tabular}

Table 3.2: Selection rules for induced electric dipole (ED) and magnetic dipole (MD) transitions taken from Ref. [106].

\subsubsection{Intensity parametrization}

Magnetic dipole transitions are usually calculated without any additional parametrization; the formulas are believed to be correct. Induced electric dipole transitions are parametrized, especially because $a b$ initio calculations do not yield reliable results. Once the dipole operator has been calculated, one can calculate the dipole strength $D$. The dipole strength $D$ is defined as the absolute square of the matrix element of the dipole operator

$$
D=\left|\left\langle i\left|\mathbf{D}_{\rho}^{(1)}\right| f\right\rangle\right|^{2}=\left|\mathbf{D}_{\rho, i \rightarrow f}^{(1)}\right|^{2}
$$

and from there the oscillator strength $P$ is calculated to be:

$$
P=\frac{8 \pi^{2} m_{e} c}{h e^{2}} \bar{\nu} D
$$

The first parametrization for these quantities related to experimental spectra was given by Judd, who derived an intensity parametrization scheme for lanthanides in solution [98]. The difference to equation 3.24 is that the system is randomly oriented and that no transitions between individual Stark levels are considered, but only the transitions between $L S J$ multiplets. The parametrization Judd obtained for the dipole strength $D$ was further modified by Axe [123].

$$
D=\frac{\chi_{e d}}{[J]} e^{2} \sum_{\lambda=2,4,6} \Omega_{\lambda} \mid\left\langle l^{N} \gamma J\left\|U^{(\lambda)}\right\| l^{N} J^{\prime}\right\rangle
$$




\subsection{LUMINESCENCE PROPERTIES OF TRIPLY IONIZED LANTHANIDES}

where $\chi_{e d}$ is the susceptibility, $U^{(\lambda)}$ are the unitarian tensor operators (in units $\mathrm{cm}^{-1}$ ) and $\Omega_{\lambda}$ are the (Judd-Ofelt) intensity parameters

$$
\Omega_{\lambda}=[\lambda] \sum_{k, q} \frac{\left|A_{k q}\right|^{2} \Xi^{2}(k, \lambda)}{[k]}
$$

with the dimension of $\mathrm{cm}^{-2}$. The physical meaning of the $\Omega_{\lambda}$ parameters is the square of the charge displacement due to induced electric dipole transitions. $5^{5}$ A large compilation of intensity parameters for lanthanides in different hosts is given in Ref. [107].

This parametrization, which represents transition intensities between multiplets, is not valid to describe intensities between individual Stark levels. Other parametrization schemes are necessary; an overview is given in ref. [107]. However, working with equation 3.32 is often sufficient to characterize individual transitions and to predict the luminescence spectra for ion implanted semiconductors in good approximation. This will be discussed at a later point in this work.

\subsubsection{The oscillator in case of emission}

Once the dipole strength is known, the Einstein coefficient for spontaneous absorption representing the transition probability, can be calculated. For emission spectroscopy the situation is different. The intensity or transition probability is proportional to the Einstein coefficient for spontaneous emission. When placing the oscillator in a dielectric medium the dipole strength $D$ has to be corrected for by the susceptibility $\chi$. For an electric dipole this correction is known as the Local Lorentz field correction. For both induced electric dipole and magnetic dipole radiation, and the case of emission spectra, the correction factors are in first order of approximation [107]:

$$
\chi_{E D}=\frac{n\left(n^{2}+2\right)^{2}}{9} \quad(\mathrm{ED}) \quad \chi_{M D}=n^{3} \quad \text { (MD) . }
$$

The Einstein coefficient for spontaneous emission between two individual non-degenerate Stark levels is then given as: ${ }^{6}$

\footnotetext{
${ }^{5}$ In contrast to electric dipole radiation, magnetic dipole radiation may be interpreted as a rotation of charge.

${ }^{6}$ This formula is just a special case of a more general formula derived for multipole radiation, known not only in atomic (see e.g. Sobel'man [109]) but, except the term accounting for the susceptibility, also in nuclear physics (see e.g. Adler and Steffen [124])
} 


$$
A(i \rightarrow f)=\frac{64 \pi^{4} \bar{\nu}^{3}}{3 h}\left[n^{3} D_{M D}+\frac{n\left(n^{2}+2\right)^{2}}{9} D_{E D}\right] .
$$

Equation 3.35 accounts for a remarkable phenomenon. As can be seen from figure 3.4 the correction factor $\chi \bar{\nu}_{0}^{3}$ which is given in curves A for a constant refractive index $n$ for $6 \mathrm{H}-\mathrm{SiC}$ and $2 \mathrm{H}-\mathrm{GaN}$, varies orders of magnitude from the infrared to the visible part of the spectrum. Therefore the same dipole strength $D$ can lead to an emission probability that is two orders of magnitude higher in the ultraviolet that in the infrared. This suggests that the implementation of UV light emitters based on lanthanide doped wide band gap semiconductors is very promising. This is of course not the whole truth. Equation 3.35 is in fact only useful to compare transitions starting from the same initial Stark level, transitions starting from different Stark levels cannot be directly compared.

Until now it has been assumed implicitly that the refractive index $n$ is constant. In fact, $n$ depends on wavelength, temperature, and in anisotropic crystals on the direction within the crystal. The two latter effects are often neglected; the first can be taken into account by e.g. the Sellmeier - type dispersion relationship [125]:

$$
n(\lambda)^{2}=A_{0}^{2}+\frac{A_{1}^{2} \lambda^{2}}{\lambda^{2}-A_{2}^{2}}
$$

In figure 3.4 the graphs labeled B show the correction factor $\chi(\lambda) \bar{\nu}_{0}^{3}$, where $n(\lambda)$ was taken, in Sellmeier - type form, from the literature. For $6 \mathrm{H}-\mathrm{SiC}$ the values are taken from ref. [126] for $2 \mathrm{H}-\mathrm{GaN}$ the values are taken from refs. [127] and [128]. As the refractive index for wide band gap semiconductors usually increases with decreasing wavelength, this will additionally increases the transition probabilities for transitions having shorter wavelengths.

\subsubsection{Radiative lifetimes and quantum efficiency}

Equation 3.37 accounts for transitions between individual Stark levels. The transition probability between an initial multiplet $i$ and a final multiplet $f$ can be derived: 


\subsection{LUMINESCENCE PROPERTIES OF TRIPLY IONIZED LANTHANIDES}

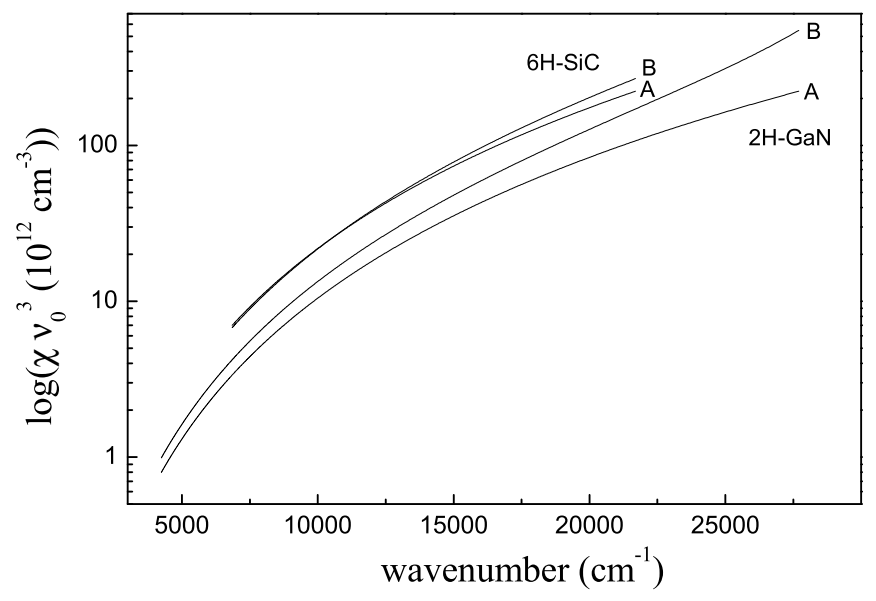

$A(i \rightarrow f)=\frac{64 \pi^{4} \bar{\nu}^{3}}{3 h\left(2 J_{i}+1\right)}\left[n^{3} D_{M D}+\frac{n\left(n^{2}+2\right)^{2}}{9} D_{E D}\right]$

where $J_{i}$ belongs to the initial level $i$. Equation 3.37 is essentially an average over the individual Stark levels of the initial multiplet. It is implicitly assumed that these Stark levels are equally populated.

The transition time from state $i$ to state $f$ is defined as

$$
\tau(i \rightarrow f)=\frac{1}{A(i \rightarrow f)}
$$

Taking into account all possible radiative channels through which a once populated level may decay, equation 3.38 turns into

$$
\tau(i)=\frac{1}{\sum_{f} A(i \rightarrow f)}
$$

which is the lifetime of level $i$. It is often found that the experimental lifetimes are not equal but
Figure 3.4: Wavelength dependent correction factor $\chi \bar{\nu}_{0}^{3}$ for an oscillator in a dielectric medium and the case of emission. The graphs labeled A show the correction for a fixed infrared refractive index $n_{\infty}$, the graphs labelled $\mathrm{B}$ for a wavelength dependent refractive index $n(\lambda)$ fit to a Sellmeier type dispersion relationship. The $n(\lambda)$ and $n_{\infty}$-values for $6 \mathrm{H}-\mathrm{SiC}$ are taken from ref. [126], the values for $2 \mathrm{H}-\mathrm{GaN}$ from refs. [127] and [128].

\footnotetext{
which is the lifetime of level $i$. It is often found that the experimental lifetimes are not equal but
} 
smaller than the calculated ones, the ratio of both being defined as the quantum efficiency $\eta: \nabla$

$$
\eta=\frac{\tau_{e x p}}{\tau_{c a l c}}
$$

Such a discrepancy, characterized by $\eta<1$, can be attributed to non-radiative processes such as energy transfer to the phonon system or, in the case of larger concentrations of ions in the host, to energy transfer between lanthanide ions.

In emission spectrosopy it is useful to introduce another quantity, the branching ratio $\beta_{i f}$ :

$$
\beta_{i f}=\frac{A(i, f)}{\sum_{i^{\prime}, f^{\prime}} A\left(i^{\prime}, f^{\prime}\right)}
$$

which is a correction factor for equation 3.37 in order to take into account all the different radiative transition paths, through which a populated state may decay. This is a very important measure to characterize lanthanides for luminescence devices. Typical examples are the transitions from ${ }^{1} G_{4}$ of $\mathrm{Tm}^{3+}$, which show that the ${ }^{1} G_{4} \rightarrow{ }^{3} H_{6}$ transition at around $480 \mathrm{~nm}$ is much less probable than the ${ }^{1} G_{4} \rightarrow{ }^{3} H_{5}$ transition at around $800 \mathrm{~nm}$. The implementation of $\mathrm{Tm}^{3+}-$ based blue light emitters is somewhat inefficient, when semiconductors are used with have a band gap such that levels above ${ }^{1} G_{4}$ are not excited, e.g. GaN.

\subsubsection{Temperature-dependent phenomena: electron phonon interaction}

Besides the influence on the host with regard to energetic positions or intensities of transitions between multiplets and Stark levels due to the crystal field, the second most dominant influence is the interaction of $4 f$ electrons and the phonons in the host matrix. This interaction is usually divided into interactions with acoustical phonons and those with optical phonons. The interaction with acoustical phonons is responsible for the fractional thermal population of Stark levels (discussed below) as well as broadening and energetic shifts of luminescence lines. In principle it may be used to determine thermodynamic quantities such as the inner energy of the host as well as vibrational properties of the lanthanide ions in the host. Because in reality this

\footnotetext{
${ }^{7}$ In fact, the quantum efficiency may reach values larger than one if a populated Stark level decays to a lower lying level which,in turn, decays to e.g. the ground state. If in both cases a photon is emitted then the quantum efficiency is larger than one. This phenomenon is known as quantum cutting, first proposed by Dexter [129].
} 


\subsection{LUMINESCENCE PROPERTIES OF TRIPLY IONIZED LANTHANIDES}

is only applicable in a few cases, namely those when both initial as well as final multiplets are not split by the crystal field (e.g. the ${ }^{5} D_{0}$ level of $\mathrm{Eu}^{3+}$ ), a discussion will be omitted here. These processes are summarized e.g. by Hüfner [105]. The interaction with optical phonons is the dominant process leading to nonradiative decay of multiplets if these are sufficiently spaced from the multiplets below to prevent relaxation due to acoustical phonons. A theoretical treatment of the probability of non-radiative transitions was given by Riseberg and Moos [130] who derived an expression for the non-radiative multiphonon transition rate $W_{n r}$ :

$$
W_{n r}=W_{0, n r}(\bar{n}-1)^{\rho}
$$

where $W_{0}$ represents the spontaneous transition rate at $T=0, p$ the order of the process equivalent to the number of phonons involved, and $\bar{n}$ the mean thermal occupation number of a certain vibrational mode, following the Bose-Einstein distribution:

$$
\bar{n}(T)=\left(\exp \left(\hbar \omega / k_{B} T\right)\right)^{-1}
$$

where $\hbar \omega$ represents the energy of the mode (phonon). An analytical expression can be derived for the temperature dependent multiphonon rate by summation over all orders of these processes, which belong to a certain phonon energy. Additionally, an empirical parameter $S_{0}$ (Huang-Rhys coupling parameter) has to be introduced, which accounts for the magnitude of the electron-phonon interaction [131]:

$$
W_{n r}(T)=B \exp \left[-S_{0}(2 \bar{n}(T)+1)\right] \sum_{j=0}^{\infty} \frac{\left(S_{0} \bar{n}(T)\right)^{j}\left[S_{0}(1+\bar{n}(T))\right]^{p+j}}{j !(p+j) !}
$$

$W_{n r}(T)$ in the above equation is hard to calculate, but there are some general tendencies to estimate whether an experimentally missing multiplet is absent because of this non-radiative decay or is simply not excited. 1) The higher the maximum phonon frequency in the host matrix the larger the probability for a non-radiative decay. Some selected examples are shown in figure 3.5. 2) Orders higher than 6 (the number of phonons involved) have not been confirmed experimentally. 3) The more covalent the bonding, the stronger the electron-phonon interaction. 


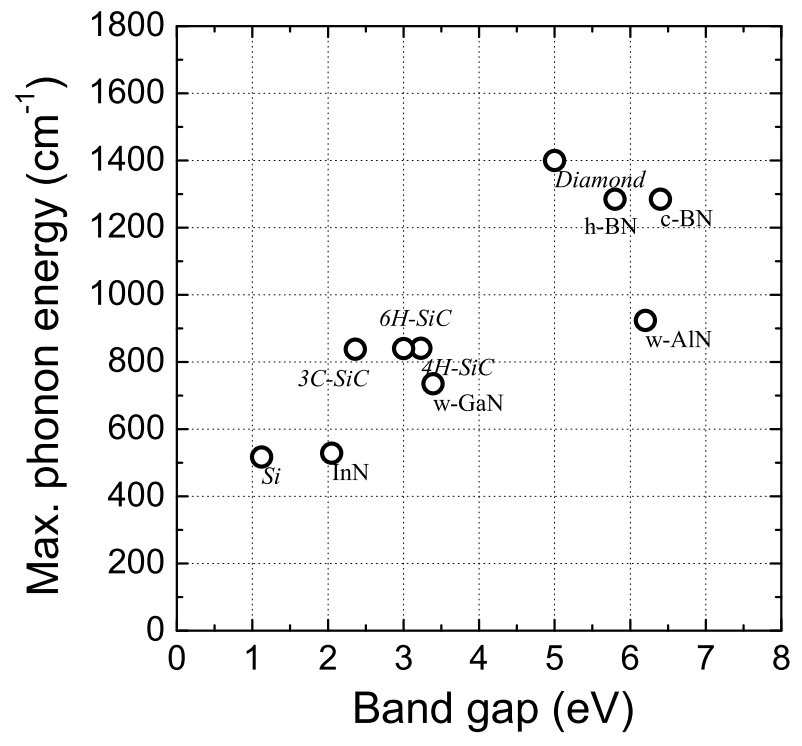

Figure 3.5: Band gap versus maximal optical phonon frequencies for a few selected semiconductors [125, 134].

4) The magnitude of the electron-phonon interaction varies over the lanthanide series and is largest at the beginning $\left(\mathrm{Ce}^{3+}, \mathrm{Pr}^{3+}\right)$ and the end $\left(\mathrm{Tm}^{3+}\right)$ and lowest in the middle $\left(\mathrm{Gd}^{3+}\right.$, $\mathrm{Eu}^{3+}$ ) [132, 133].

The additional non-radiative transition probabilities, indicated in equation 3.42, lead to a characteristic temperature dependence of the lifetime of multiplets (compare equation 3.49) but only in some cases multi-phonon relaxations are the sole origin of temperature dependent lifetimes. An additional temperature dependence is introduced by the fractional thermal population and will be discussed in the next chapter.

\subsubsection{Fractional thermal population}

Each populated multiplet, which is sufficiently spaced from the neearest lower lying multiplet to prevent non-radiative depopulation, and which is split by the crystal field, interferes with acoustical phonons in such a way that the population of each level is determined by the Boltzmann distribution. This is only valid if the excited multiplets possess a sufficiently long lifetime and thermal equilibrium can be assumed. For $4 \mathrm{f}$ electron systems the lifetime is generally in the range of ms to ns, whereas the electron-phonon interaction occurs at a time scale of less than 1 ps [105]. The fractional thermal population $X(A)$ of a single Stark level $A$ within the multiplet is then given by 


\subsection{LUMINESCENCE PROPERTIES OF TRIPLY IONIZED LANTHANIDES}
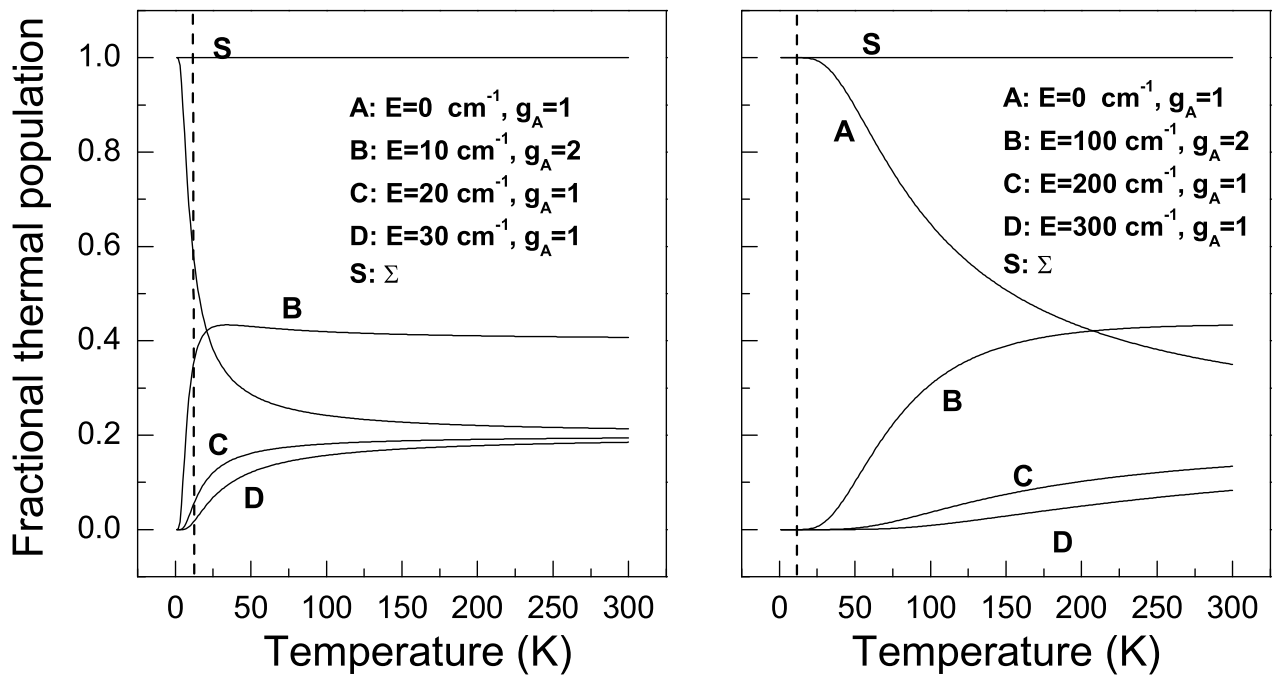

Figure 3.6: Example for fractional thermal populations, typically found in $S L J$ multiplets split by a crystal field. Left: example with four Stark levels at $0 \mathrm{~cm}^{-1}$ (A), $10 \mathrm{~cm}^{-1}$ (B), $20 \mathrm{~cm}^{-1}$ (C) and 30 $\mathrm{cm}^{-1}$ (D), right: example with four Stark levels at $0 \mathrm{~cm}^{-1}$ (A), $100 \mathrm{~cm}^{-1}$ (B), $200 \mathrm{~cm}^{-1}$ (C) and 300 $\mathrm{cm}^{-1}$ (D). In both cases the second lowest Stark level is assumed to be two-fold degenerate, all other levels are one-fold degenerate.

$$
X(A)=\frac{g_{A} e^{\frac{-\left(E_{A}-E_{0}\right)}{k_{B} T}}}{\sum_{i} g_{i} e^{\frac{-\left(E_{i}-E_{0}\right)}{k_{B} T}}}
$$

where $g_{A}$ is the degeneracy of level $A$ and $E_{A}$ the energy of level $A$ above the energy $E_{0}$ of the lowest Stark level. The sum in equation 3.44 runs over all levels of the multiplet. For typical splittings encountered in multiplets, which are due to the crystal field, this is illustrated in figure 3.6. There is an important consequence resulting from the fractional thermal population: the lifetime of a Stark level belonging to a multiplet split by the crystal field always reflects the lifetime of the whole multiplet, and this lifetime is temperature dependent. The lifetime of an arbitrary $S L J$ multiplet, measured at transitions starting from one or more of its Stark levels, can be written as follows: 


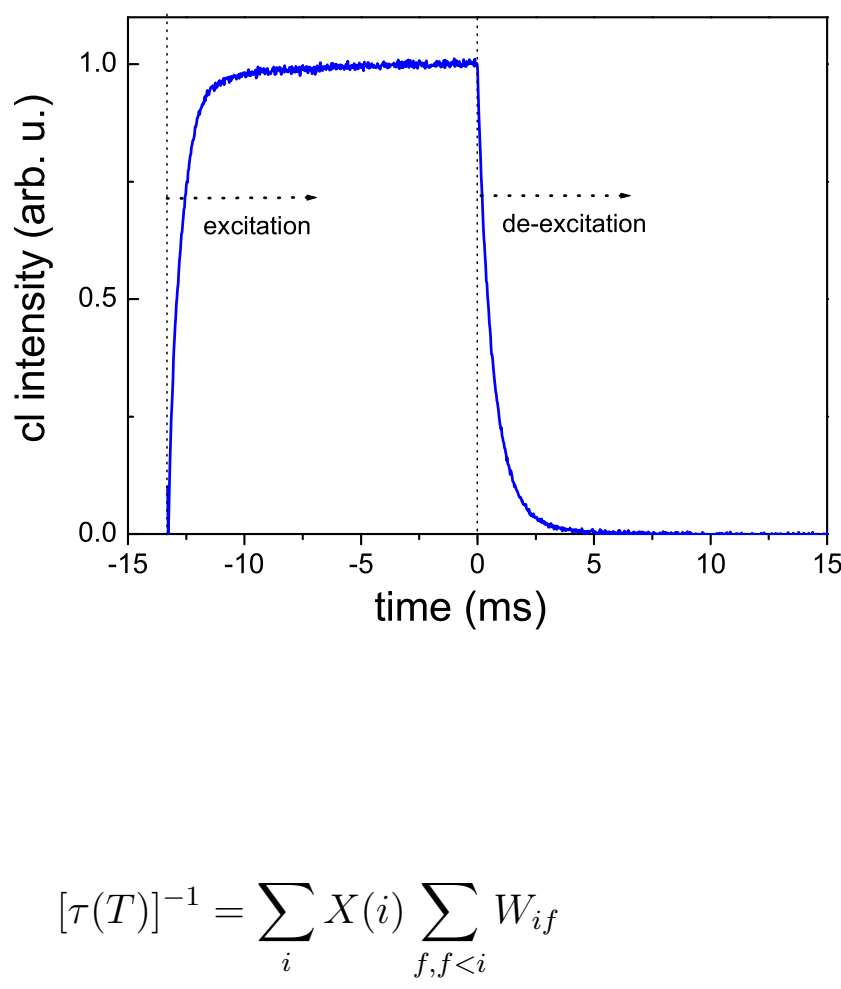

Figure 3.7: Measurement of excitation and deexcitation of $\mathrm{Ln}^{3+}$ luminescence.

where $X(i)$ is the fractional thermal population as described in equation 3.44, $W_{i f}$ is the Einstein coefficient for spontaneous emission from level $i$ to level $f$, as shown in equation 3.35 , and $\nu_{i f}$ is the energy difference between any two arbitrary Stark levels, for which $E_{i}>E_{f}$.

\subsubsection{Relaxation phenomena: time-resolved spectroscopy}

Studying the excitation and relaxation of luminescence due to intra-4f electron transitions generally gives a deeper insight into mechanisms involved in excitation. A typical time-resolved intensity spectrum is shown in figure 3.7, where the excitation and the deexcitation time period is shown. The curve is described by integral solutions to appropriate rate equations which account for the possible excitation and deexcitation mechanism. For all energy levels of a certain lanthanide this leads to a general set of rate equations

$$
\dot{N}(t)=A N(t)
$$

which can be solved according to methods described in the literature (see e.g. Ref. [135] (in 


\subsection{LUMINESCENCE PROPERTIES OF TRIPLY IONIZED LANTHANIDES}

German)). Experimental spectra, such as the one shown in figure 3.7, are linked to equation 3.46 in the following manner:

$$
I(t)-I_{B G}=-\frac{d N_{i}(t)}{d t}
$$

where $I_{B G}$ accounts for the experimental background.

In its simplest form, the deexcitation curve in Figure 3.7 is a single exponential function described by the rate equation

$$
d N / d t=-\tau^{-1} N
$$

and the excitation curve by the rate equation

$$
d N / d t=-\tau^{-1} N+\sigma I\left(N_{0}-N\right)
$$

If the particle flux $I$ is known, the excitation cross section $\sigma$ can be determined directly from the curve. This simple approach is often sufficient [136, 137, 69], but the formulation of the corresponding rate equations may get complicated [136].

In a quite practical approach, the most important deexcitation mechanisms with respect to the expected decay curves, will be summarized.

Single exponential decay: In the case of $n$ radiative or nonradiative decay channels, the overall transition probability $W_{\text {tot }}$ is given by

$$
\frac{1}{\tau}=W_{t o t}=W_{1}+W_{2}+\ldots . W_{n}
$$

of which the integral solution simply is 


$$
N(t)=N_{0} \cdot e^{-\frac{t}{\tau}} .
$$

This includes transitions from individual Stark levels of a multiplet, additional multi-phonon relaxation or other deexcitation mechanisms, such as the Auger backtransfer process.

N-exponential decay: Exponential decays, which are simply a sum of independent decays of the form

$$
N(t)=\sum_{i} N_{0, i} \cdot e^{-\frac{t}{\tau_{i}}}
$$

where $N=\sum_{i} N_{i}$, are found if the luminescence light originates from ions on different sites. A typical example is an amorphous local environment.

Complex decays and energy transfer: Any deviation from a single-exponential decay is unambiguously attributable to energy transfer to the $4 f$-electron system after the excitation source is turned off, provided only a single local environment is present. In this case, a decay of the types described above can be excluded. Typical examples are the energy transfer between different lanthanide ions. Another example would be the population of a multiplet by another multiplet, which is energetically located above, but within the same ion. Finally, the involvement of particles differing from the incident ones (such as photons or electrons) in the excitation process, typically excitons, would fall into the same category.

Although excitons often possess lifetimes too short to contribute significantly to the deexcitation spectra with respect to the long-lived intra-4f luminescence, both the population from higher lying levels and the energy transfer between lanthanide ions may be well visible in the decay. The population of the levels is treated in terms of the set of rate equations 3.46, an example can be found in this work in chapter 5.1.4 The energy transfer processes were first investigated by Förster [138] and Dexter [139]. Inokuti and Hirayama [140] modified the theory to account for energy transfer between $4 f$-shells of triply ionized lanthanides. They showed that the decay luminescence can be described by the formula ${ }^{8}$

\footnotetext{
${ }^{8}$ One should keep in mind that the formula derived by Inokuti and Hirayama is only an approximation, based on a model for energy diffusion.
} 


$$
I(t)=I_{0} \cdot \exp \left[-\frac{t}{\tau_{0}}-\Gamma\left(1-\frac{3}{s}\right) \frac{c}{c_{0}}\left(\frac{t}{\tau_{0}}\right)^{3 / s}\right]
$$

where $\tau_{0}$ is the lifetime, $\Gamma$ is the Gamma-function, $s$ describes the type of interaction ( $s=6$ for dipole-dipole interaction, $s=8$ for dipole-quadrupole interaction, $s=10$ for quadrupolequadrupole interaction and $s=\ln |z|$ for exchange interaction), $c$ is the concentration of the acceptors and $c_{0}$ stands for the critical transfer radius. Many of the luminescence decays in this work are of the type described by equation 3.51 . One consequence of the energy transfer, self-quenching [141], which increases with increasing lanthanide concentration in the host, is a well-known problem not only in the field of lanthanide doped solid state lasers [102].

A good overview of possible decay curves and underlying models can be found for the twolevel system $\mathrm{Si}^{2} \mathrm{Er}^{3+}$ [3, 64, 142, 4, 143].

\subsubsection{Numerical details and extensions}

To calculate the energy levels of the free triply ionized lanthanides as well as the interaction between the $4 f$-electron system and the surrounding crystal field, a software package was used which was developed and kindly supplied by Dr. Michael F. Reid [144]. This commandline oriented software package allows the calculation of the energy matrix and corresponding eigenvectors, the expectation of several operators as well as intensity calculations based on either the parametrization scheme of Axe or on the Reid-Richardson intensity model [145]. The software package was designed for the analysis of absorption spectra. As mentioned ealier the calculations are mainly based on the parametrization of each of the interactions, which in turn are ascribed to the reduced matrix elements. A general matrix element of any of the freeion interactions $\mathbf{H}_{i}$ takes the form [146]

$$
\left\langle\gamma_{1} S L J\left|\mathbf{H}_{i}\right| \gamma_{2} S^{\prime} L^{\prime} J^{\prime}\right\rangle=H_{i} \delta_{J, J^{\prime}} c\left(S L S^{\prime} J^{\prime}\right)\left\langle\gamma_{1} S L\left\|h_{i}\right\| \gamma_{2} S^{\prime} L^{\prime}\right\rangle
$$

where $H_{i}$ are the parameters of the interaction, $c\left(S L S^{\prime} J^{\prime}\right)$ is a numerical constant and $\langle\|\|\rangle$ is the squared reduced matrix element, calculated with the help of the tables of Nielson and Koster [147]. The eigenvectors obtained in the calculations are of the form 


$$
\Psi\left({ }^{2 S+1} L_{J}\right)=\sum_{\gamma S L} a_{\gamma S L J}|\gamma S L\rangle .
$$

The crystal field matrix element is included in the same manner, but additionally the mixing of levels with different $J$-values is accounted for. The energy matrix is then characterized by individual blocks of energy levels which correspond to submatrices of the energy matrix. Each of these submatrices can be related to an irreducible representation or the crystal field quantum number $\mu$. Each of the energy levels is then described by a pair of numbers reflecting the block as well as the level within this block. A more detailed description of the numerical technique is given by Liu [146]. The degeneracy of a $4 f$ electron system may be very high, and therefore corresponding energy matrices can be very large. In this case, one may work with a truncated set of eigenvectors, including only a subset of eigenfunctions. Because the energy matrix can be blocked out into a set of submatrices, only a part of the energy matrix has to be evaluated. In the case of $\mathrm{Tm}^{3+}$, where the dimension of the energy matrix is only $(91)^{2}$, one may work with the full set of eigenvectors. For $\mathrm{Eu}^{3+}$ or $\mathrm{Gd}^{3+}$ this is often not applicable and the use of truncated sets of eigenfunctions is recommendable to reduce computing time.

To evaluate absorption spectra the usual approach is to assign transitions between individual Stark levels to absolute energy values of the corresponding eigenvalues belonging to these Stark levels, measured preferentially at a low temperature. This is reasonable if the transition starts from the lowest Stark level of the ground state multiplet, whose energy eigenvalue is set to zero, without loss of generality. In luminescence spectroscopy, where transitions between individual Stark levels do not necessarily involve the same Stark level or multiplet, a transition between two Stark levels cannot be characterized with only one block/level pair any longer, two pairs are needed instead. This was the first extension to the software package. An arbitrary transition with a certain energy is then indicated as follows:

energy block1 level1 block2 level2.

The second extension to the software package was the implementation of a more powerful fitting algorithm than the least-squares fit, namely the differential evolution algorithm. Instead of using linear corrections as done in least squares fitting, this fitting routine diagonalizes and solves the energy matrix, including free ion and crystal field interactions during each fitting step. The differential evolution algorithm is a global optimization method invented by Storn and Price [148]. It is also included in the SPECTRA software provided at the Argonne National Laboratory for evaluation of experimental lanthanide Stark level spectra. A complete descrip- 


\subsection{LUMINESCENCE PROPERTIES OF TRIPLY IONIZED LANTHANIDES}

tion of the implementation cannot be given in the scope of this thesis and the reader is referred to the corresponding documentation [149] (in German).

Many of the calculations in this work were performed with the software mentioned above, ported to a DEC Alpha $433 \mathrm{MHz}$ workstation running under Linux. The diagonalization of the energy matrix was, except for the large matrices, performed in reasonable times. The final crystal field calculations were undertaken at the Army Research Lab/Adelphi Laboratory Center, Maryland, USA by Dr. Bahram Zandi using a large computational facility.

\subsection{8 $\mathbf{E u}^{3+}, \mathbf{T m}^{3+}$ and $\mathbf{G d}^{3+}$}

In chapter 3.1.2.5 the computation of the Dieke diagram for the whole lanthanide series was shown. If one is interested in the implementation of light emitters emitting a certain colour or wavelength, attention should focus on those energy levels sufficiently spaced from lower lying levels to prevent non-radiative decay. Additionally, the knowledge of the branching ratios is needed (or at least the squared reduced matrix elements should be considered), for all emissions starting from these levels. This leads to the preferable element for the implementation of a light emitter of a certain wavelength or colour. Typical examples of visible light emission are $\mathrm{Eu}^{3+}$ $\left({ }^{5} D_{0} \rightarrow{ }^{7} F_{2}\right.$ : red at $\left.620 \mathrm{~nm}\right)$ and $\mathrm{Tm}^{3+}\left({ }^{1} G_{4} \rightarrow{ }^{3} H_{6}\right.$ : blue at $\left.485 \mathrm{~nm}\right)$. In order to perform a crystal field analysis, a larger number of sufficiently spaced energy levels is needed, especially because it is important to monitor different multiplet to multiplet transitions ending up at the same multiplet. In principle, these criteria rule out the ions $\mathrm{Ce}^{3+}, \mathrm{Eu}^{3+}$ and $\mathrm{Yb}^{3+}$. Additionally the crystal field splitting should be large enough. The only critical ion in this case is $\mathrm{Gd}^{3+}$ where the ground state splitting is so small that in general it cannot be resolved in luminescence spectroscopy, although on the other hand this might be an advantage, as shown in chapter 5.1.2. A last point that should be considered is the fact than the lifting of degeneracy in a crystal field is more advanced for even-numbered electron systems. For a crystal field analysis this limits the ions of interest to $\mathrm{Pr}^{3+}, \mathrm{Tb}^{3+}, \mathrm{Ho}^{3+}$ and $\mathrm{Tm}^{3+}$, where $\mathrm{Ho}^{3+}$ is less preferable because of the high $\mathrm{J}-$ value of the ${ }^{5} I_{8}$ ground state.

In this work the focus will be on the ions $\mathrm{Tm}^{3+}, \mathrm{Gd}^{3+}$ and $\mathrm{Eu}^{3+}$. Usually, one would chose $\mathrm{Pr}^{3+}$ over $\mathrm{Tm}^{3+}$ for a crystal field analysis, because the upper ${ }^{1} S_{0}$ level of $\mathrm{Tm}^{3+}$ is, in contrast to $\mathrm{Pr}^{3+}$, far above the largest band gaps of known semiconductors. However, the fact that up to now no crystal field analysis exists for $\mathrm{Tm}^{3+}$ in wide band gap semiconductors makes it the preferable choice. In addition, $\mathrm{Eu}^{3+}$ will be included in the discussion, because it is by far the most frequently investigated element in insulators. $\mathrm{Gd}^{3+}$ will be investigated in the AlN host in addition to $\mathrm{Tm}^{3+}$ and $\mathrm{Eu}^{3+}$. 
In the following each of the ions $\mathrm{Eu}^{3+}, \mathrm{Tm}^{3+}$ and $\mathrm{Gd}^{3+}$ will be described and selected references, which helps to understand and compare the luminescence spectra of these ions in the semiconductor hosts, will be given. Figure 3.1 .8 gives a classification of these ions with regard to the squared reduced matrix elements $\left\|U^{\lambda}\right\|^{2}$ between the ground state and the most important excited states. The corrected squared reduced matrix elements, $\left\|U^{\lambda}\right\|^{2} \bar{\nu}^{3}$, relevant for emission, are also shown. This classification allows to see which levels can be excited if the nature of the excitation mechanism is e.g. impact excitation, and it shows for which levels one may expect luminescent transitions to the ground state. This classification is, of course, incomplete because one has to take into account all possible transitions, but this may result in huge lists of reduced matrix elements.

\section{$\mathbf{E} \mathbf{u}^{3+}$}

$\mathrm{Eu}^{3+}$ has become the standard probe in absorption as well as in luminescence spectroscopy. This is due to its low $J$-values of the ground state ${ }^{7} F_{0}$ and first excited state ${ }^{7} F_{1}$. Another reason for this is a well-spaced ${ }^{5} D_{0}$ level at about $17,200 \mathrm{~cm}^{-1}$, which is separated by more than 6,000 $\mathrm{cm}^{-1}$ from the next lower lying level ${ }^{7} F_{6}$ (see figure 3.2). The squared reduced matrix elements between the most important levels as regards luminescence spectroscopy and the ground state are shown in figure 3.1.8. In contrast to $\mathrm{Tm}^{3+}$, which will be discussed below, these matrix elements are rather small. Experimental and calculated branching ratios predict that the ${ }^{5} D_{0}$ level most probably decays to the ${ }^{7} F_{2}$ level, giving rise to luminescence at around $620 \mathrm{~nm}$. A huge number of experimental data exists (see e.g. the compendia by Morrison and Leavitt [100] and Görller-Walrand and Binnemans [106]), and often new materials are investigated with $\mathrm{Eu}^{3+}$ first. Typical applications of Eu-doped materials are spectral hole burning [150] and luminescent phosphors, like in the case of the euro currency [151].

With increasing band gap of available semiconductors, luminescence investigations were applied to materials implanted with europium, such as GaN [35], AlN [43] as well as BN [51].

\section{$\mathbf{T m}^{3+}$}

$\mathrm{Tm}^{3+}$ has the ground state configuration ${ }^{3} H_{6}$ and, among the triply ionized lanthanides, a sufficient number of levels which are energetically far above the next lower lying levels. The level scheme of $\mathrm{Tm}^{3+}$ is shown in figure 3.2. calculated on the basis of the mean free ion parameters (see table A.1). All but the ${ }^{1} S_{0}$ level are located below $50,000 \mathrm{~cm}^{-1}$. The levels ${ }^{1} I_{6},{ }^{1} D_{2}$, ${ }^{1} G_{4},{ }^{3} H_{4},{ }^{3} H_{5}$ and ${ }^{3} F_{4}$ are sufficiently spaced from the next lower lying levels to prevent nonradiative multiphonon decay. This makes $\mathrm{Tm}^{3+}$ in principle the ideal probe for luminescence 


\subsection{LUMINESCENCE PROPERTIES OF TRIPLY IONIZED LANTHANIDES}

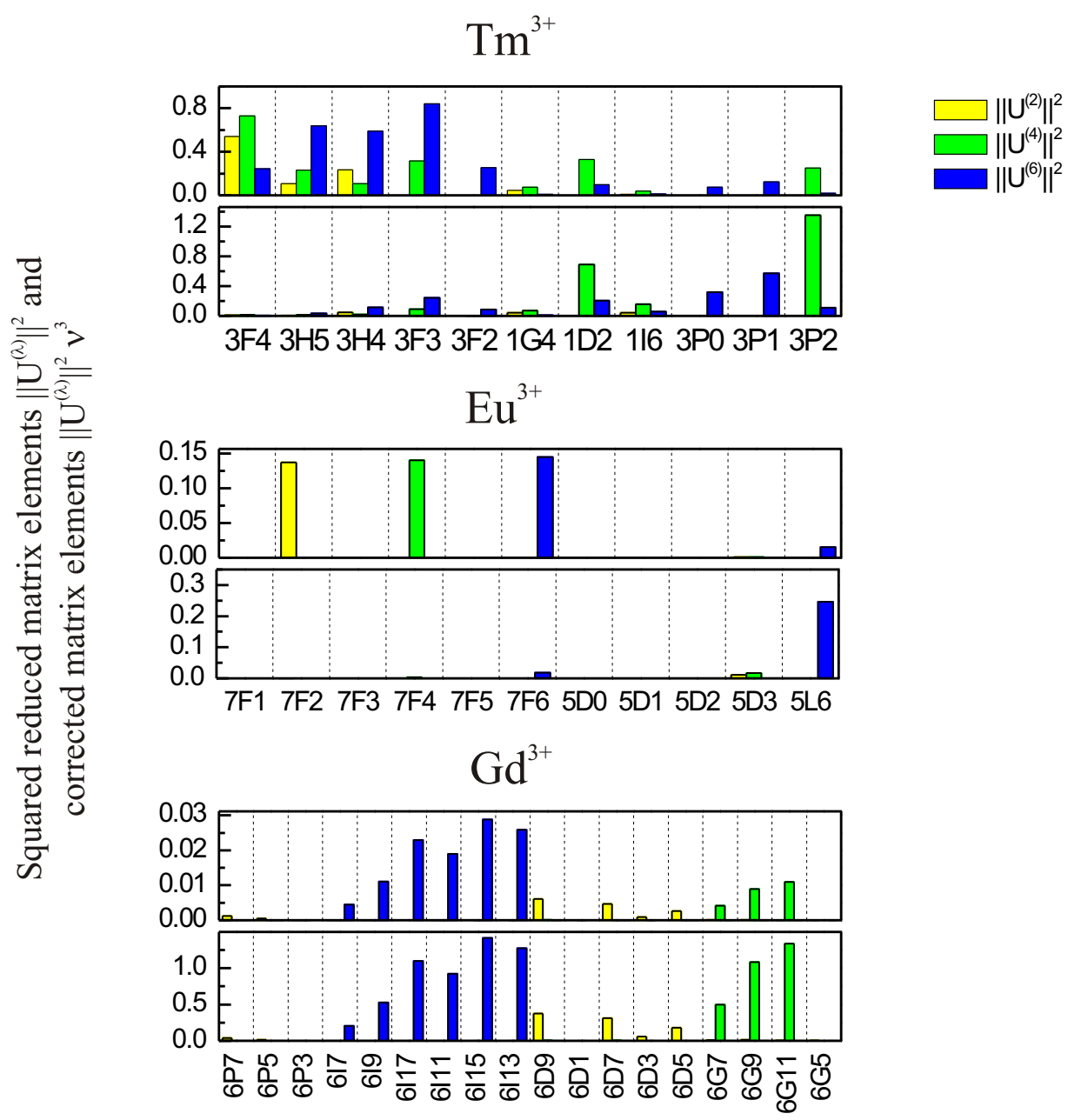

Figure 3.8: Selected squared reduced matrix elements $\left\|U^{\lambda}\right\|^{2}$ (top) and corrected matrix elements $\left\|U^{\lambda}\right\|^{2} \bar{\nu}^{3}$ (bottom) for transitions between the excited levels and the ground state for $\mathrm{Tm}^{3+}, \mathrm{Eu}^{3+}$ and $\mathrm{Gd}^{3+}$. The values for $\left\|U^{\lambda}\right\|^{2}$ are given in $\mathrm{cm}^{-1}$, the values for $\left\|U^{\lambda}\right\|^{2} \nu^{3}$ in $10^{13} \mathrm{~cm}^{-4}$ for $\mathrm{Tm}^{3+}$ and $10^{12} \mathrm{~cm}^{-4}$ for $\mathrm{Eu}^{3+}$ and $\mathrm{Gd}^{3+}$. For $\mathrm{Tm}^{3+}$ all levels are shown, for $\mathrm{Eu}^{3+}$ the most important lower levels and for $\mathrm{Gd}^{3+}$ all levels below $50,000 \mathrm{~cm}^{-1}$. The notation of the $\mathrm{Gd}^{3+}$ levels is given as ${ }^{2 S+1} L_{2 J}$.

investigations in the case of relatively large band gaps, which is the main reason for its choice as a luminescence probe in this work. The main applications of $\mathrm{Tm}^{3+}$ are infrared to blue upconversion materials [152, 153, 154, 155, 156, 157] and solid state lasers (see e.g. the overview given by Kaminskii [102]). In contrast to e.g. $\mathrm{Eu}^{3+}$ and $\mathrm{Tm}^{3+}$, the squared reduced matrix elements between excited levels and the ground state are all very large (see Figure 3.1.8) making $\mathrm{Tm}^{3+}$ a very promising candidate for blue and near-UV light emitting devices. Useful energy levels calculations and luminescence spectra can be found for a variety of different materials, such as thulium doped $\mathrm{Ca}_{4} \mathrm{GdO}\left(\mathrm{BO}_{3}\right)_{3}$ [158], Tm ${ }^{3+}: \mathrm{NaLa}\left(\mathrm{MoO}_{4}\right)_{2}[159]$ and $\mathrm{Tm}^{3+}: \mathrm{Y}_{2} \mathrm{Al}_{5} \mathrm{O}_{12}$ 
[160]. A detailed and very useful description of the energy matrix and lifetime calculations based on absorption data was given by Lakshman et al. [161] for $\mathrm{Tm}^{3+}$ in nitrate complexes.

A larger number of publication exists on Tm-implanted semiconductors, such as BN [51], AlN [50], c-BN [52] and c-BN thin films [53], to name only the wider band gap semiconductors.

\section{$\mathbf{G d}^{3+}$}

$\mathrm{Gd}^{3+}$ is relatively unique among the triply ionized lanthanides with regard to the energy difference between the ${ }^{8} S_{7 / 2}$ ground state and the first excited state ${ }^{6} P_{7 / 2}$. The level scheme for energies below $50,000 \mathrm{~cm}^{-1}$ is shown in figure 3.2 . The squared reduced matrix elements between transition and ground state, calculated using the eigenvectors corresponding to the mean free ion parameters, are visualized in figure 3.1.8. The expected transition probability for the ${ }^{6} P_{7 / 2} \rightarrow{ }^{8} S_{7 / 2}$ transition is relatively small (see figure 3.1.8), but still reasonable compared to many of the transition probability for higher lying levels than ${ }^{6} P_{7 / 2}$ to the ground state. An example is the ${ }^{4} G_{7 / 2}$ level, which may decay to the ${ }^{6} P_{J}$ or ${ }^{6} I_{J}$ levels rather than to the ground state, giving rise to a luminescence at around $600 \mathrm{~nm}$ or in the infrared (see Refs. [162, 163]). Another outstanding property of $\mathrm{Gd}^{3+}$ is the very small crystal field splitting of the ground state, which in many cases cannot be resolved (see e.g. the spectroscopic data listed by Morrison and Leavitt [100] and other luminescence spectroscopy studies [164, 162, 165, 166]). A typical example is given in this work (see chapter 5.1.2].

A promising application of $\mathrm{Gd}^{3+}$-doped materials is the implementation of quantum cutters [162,163] and light emitters operating down to the very ultraviolet region of the electromagnetic spectrum [167]. 


\subsection{ELECTRON EMISSION CHANNELING}

\subsection{Electron emission channeling}

Emission channeling can be described as the emission and movement of charged particles through a single crystal subsequent to radioactive decay. These particles are alpha and beta particles as well as conversion electrons. The emission channeling technique is the detection of these particles outside the crystal with a detector. The theoretical description of the particles' movement is classical for alpha particles as well as positrons, and quantum mechanically for electrons. As all isotopes used in this work are conversion electron and/or $\beta^{-}$emitters the discussion shall be restricted to the treatment of the channeling effect of electrons.

\subsubsection{Solutions to the Schrödinger equation}

Conversion electrons and $\beta^{-}$particles, emitted in radioactive decay, usually have kinetic energies of more than a few $\mathrm{keV}$. Therefore they are relativistic particles and their movement is described by the so-called Klein-Gordon-equation. However several conditions exist which allow a simplification of the description, i.e. a reduction of the problem so that it can be described by the time-independent Schrödinger equation. It is evident that the potential, which arises from the atoms in the crystal lattice, is much smaller than the overall energy of the electron. Furthermore, it is constant in time if the continuum model is applied where the potential is replaced by its time average. It can be simplified further by separating the movement of the electron into two independent components, one perpendicular to the direction of motion of the electron, the second one parallel to this direction. Further, it is demanded that the direction of motion coincides with an atomic row, i.e. a certain axis within the crystal, which in the following will be called the principal or $\mathrm{z}$-axis. Two independent equations of motion are obtained, the remaining Schrödinger-equation for the movement of the electron perpendicular to the $\mathrm{z}-$ axis is given by [168]:

$$
\left[-\frac{\hbar}{2 m} \Delta_{r}+V(\mathbf{r})\right] u_{j}(\mathbf{r})=E_{j} u_{j}(\mathbf{r})
$$

with the Bloch-wavefunctions $u_{j}(\mathbf{r})=\mathbf{e}^{\mathbf{i} \mathbf{k}_{\mathbf{t}} \mathbf{r}} \omega_{\mathbf{j}}(\mathbf{r})$. In order to obtain the thermally averaged potentials in the continuum approximation, the potentials are averaged taking into account the Gaussian-like probability for the elongation of the atoms, in the z-direction for the axial as well as xy-direction for the planar continuum potentials. To solve the Schrödinger equation, it is convenient to expand the wave functions $\omega_{j}(\mathbf{r})$ as well as the thermally averaged potentials (as 
shown in equation 3.55 into a Fourier row:

$$
V_{t h}(\mathbf{r})=\sum_{j} \sum_{n m} V_{j, n m}^{t h} e^{i \mathbf{g}_{j, n m} \mathbf{r}}
$$

where $j$ runs over all atoms within the two-dimensional unit cell. Using the parametrization of the atomic potential as suggested by Doyle and Tuner [169] as well as the Born approximation, the atomic scattering factors in the expansion of the potential are written as a sum of four Gaussian functions. The Fourier components may then be written as shown for the axial potential:

$$
V_{j, n m}^{t h}=2 \pi N a_{0} e^{2} e^{-\frac{1}{2} g_{j, n m}^{2} \rho^{2}} \sum_{i=1}^{4} \frac{a_{i, j}}{b_{i}, j+2 \rho^{2}} e^{-r^{2} /\left(b_{i}, j+2 \rho^{2}\right)} .
$$

The Doyle-Turner coefficients $a_{i}$ and $b_{i}$, tabulated in the literature [169], are parameters that are fitted against relativistic Hartree-Fock-calculations.

To find the exact solution of the Bloch wavefunctions, they are expanded into their eigenfunctions

$$
\mathbf{u}(z, \mathbf{r})=\sum_{j} \alpha_{j} u_{j}(\mathbf{r}) e^{-i E_{j} z / \hbar \nu}=e^{i \mathbf{k}_{t} \mathbf{r}} \sum_{j} \alpha e^{-i E_{j} z / \hbar \nu} \sum_{n m} C_{n m}^{j} e^{i \mathbf{g}_{n m} \mathbf{r}}
$$

The boundary conditions are determined by the fact that, at the surface of the crystal $(\mathrm{z}=0)$, the wave functions have to fit to an incoming or outgoing plane wave. The coefficients then satisfy the conditions $\sum_{j}\left|\alpha_{j}\right|^{2}=\sum_{j}\left|C_{00}^{j}\right|^{2}=1$ and $\alpha_{j}=C_{00}^{j}[168]$.

The normalized scattering yield is given by the absolute square value of the Bloch wave function at any position in the crystal $\mathbf{u}(z, \mathbf{r})$, also $P(z, \mathbf{r})=|\mathbf{u}(z, \mathbf{r})|^{2}$. This leads to

$$
P(z, \mathbf{r})=|\mathbf{u}(z, \mathbf{r})|^{2}=\sum_{i j} C_{00}^{i} C_{00}^{j} e^{i\left(E^{i}-E^{j}\right) z \hbar / \nu} \sum_{n m} \sum_{n^{\prime} m^{\prime}} C_{n m}^{i} C_{n^{\prime} m^{\prime}}^{j} e^{i\left(\mathbf{g}_{n m}-\mathbf{g}_{n^{\prime} m^{\prime}}\right) \mathbf{r}}
$$

Finally, the lattice vibrations of the emitter atoms can be taken into account by folding the position $\mathbf{r}$ of the emitter atom with a Gaussian function. This leads to a Debye-Waller-like term 
in equation 3.58 [170]:

$$
P(z, \mathbf{r})=|\mathbf{u}(z, \mathbf{r})|^{2}=\sum_{i j} \ldots \sum_{n m} \sum_{n^{\prime} m^{\prime}} \ldots e^{i\left(\mathbf{g}_{n m}-\mathbf{g}_{n^{\prime} m^{\prime}}\right) \rho_{I}^{2}}
$$

Here $\rho_{I}^{2}$ is the one-dimensional mean vibration amplitude of the emitter atom which can be calculated in good approximation according to [171]:

$$
\Theta_{\text {Emitter }}=\sqrt{\frac{M_{\text {Host }}}{M_{\text {Emitter }}}} \Theta_{\text {Host }}
$$

This is equivalent to an adjustment of the vibrational amplitude of the emitter atom to the one of the surrounding ligands, by assuming that the binding force between ligand and impurity atom is equal to the force between two host atoms. 9

\subsubsection{Dechanneling}

So far the movement of the electron through the crystal lattice has been assumed to be undisturbed. There are, however, several effects that cause disturbance of the ideal movement, namely the scattering at (quasi-)particles in the lattice or at lattice defects. These perturbations are denoted as dechanneling. In principle, these calculations are somewhat similar to calculations performed for estimating the range of primary electrons used as excitation sources for cathodoluminescence. It is worth noting at this point that such energetic electrons are elsewhere denoted as hot electrons in contrast to e.g. free carriers with kinetic energies below that of the host lattice; their theoretical treatment differs significantly [125].

Thermal incoherent scattering at phonons: Channeling of electrons occurs in the vicinity of the lattice atoms. Therfore the lattice vibrations will interfere with the electron. This effect is called thermal incoherent scattering at phonons, and it is equivalent to a change of momentum and state of the electrons. The cross section for a plane wave calculated in Born-approximation was given by Andersen et al. [172, 173]:

\footnotetext{
${ }^{9}$ In fact, the vibrational amplitude of the emitter atoms is often treated as an adjustable parameter in the simulations.
} 


$$
\sigma_{i n c}=2 \alpha^{2} \beta^{-2} \pi a_{0}^{2} \int_{0}^{\infty}|f(g)|^{2}\left(1-e^{-g^{2} \rho^{2}}\right) g d g
$$

$\alpha$ is the fine structure constant, $\beta=v / c, g$ is a reciprocal lattice vector and $f(g)$ the electronic scattering factor. The mean free path length is therefore given by $\lambda_{j}=\frac{1}{N \sigma_{i n c}}$. To account for the fact that the electron was described as a plane wave, a correction factor $\left|u_{j}(r=0)\right|_{t h}^{2}$ is introduced which is the electron density folded with the thermal vibration including a Gaussian function. 10

The mean free path length of an electron in the state $j$ is then given by [170]:

$$
\lambda_{j}=\frac{1}{\left|u_{j}(0)\right|_{t h}^{2} N \sigma_{i n c}} .
$$

Electronic Scattering: Electronic scattering denotes the interaction of an electron with the electron gas, the valance electrons or the trapping of the electron at an atom. This effect can again be taken into account by introducing a mean free path length $\lambda_{e l}$, which was calculated by Lervig et al. [174]:

$$
\frac{1}{\lambda_{e l}}=\omega_{0} \frac{e^{2}}{2 \hbar \nu^{2}} \ln \frac{4 m v^{2}}{\hbar \omega_{0}}
$$

$\omega_{0}$ is the plasmon frequency $\omega_{0}=\sqrt{4 \pi e^{2} n / m_{0}}$. The mean free path lengths are typically in the range of $100 \mathrm{~nm}$ for electrons with an energy between $100 \mathrm{keV}$ and $200 \mathrm{keV}$. Electronic scattering is therefore negligible as long as the atomic number $Z$ is not too small, which would decrease the cross section of the thermal incoherent scattering $\sigma_{i n c}$ significantly.

Scattering at lattice defects: Because real single crystals contain a number of defects, scattering of the electrons at these defects also influences the movement of the electron through

${ }^{10}$ Such a completely localized interaction is a rough approximation because it pronounces the scattering at the strongly localized 1s-state. 


\subsection{ELECTRON EMISSION CHANNELING}

the lattice. Typical defects are the ones created during implantation, namely impurities and vacancies. It is difficult to take these effects into account, but on the other hand the emission channeling method can be used to follow the annealing behavior of such defects if they are caused by the implantation. Yu and Cardona [125] give an introduction to the possible ways of treating the scattering at impurities theoretically.

Taking into account the thermal incoherent scattering, equation 3.59 can be written as follows:

$$
\begin{aligned}
P(z, \mathbf{r})=|\mathbf{u}(z, \mathbf{r})|^{2} & =\sum_{i}\left|C_{00}^{i}\right|^{2}\left(1-e^{-z / \lambda_{i}}\right) \\
& +\sum_{i j} C_{00}^{i} C_{00}^{j} e^{1 / 2\left(1 / \lambda_{i}+1 / \lambda_{j}\right)} e^{i\left(E^{i}-E^{j}\right) \hbar / \nu} \times I_{n m n^{\prime} m^{\prime}}^{i j}
\end{aligned}
$$

with the coefficients $I_{n m n^{\prime} m^{\prime}}^{i j}$ defined as:

$$
I_{n m n^{\prime} m^{\prime}}^{i j}=\sum_{n m} \sum_{n^{\prime} m^{\prime}} C_{n m}^{i} C_{n^{\prime} m^{\prime}}^{j} e^{i\left(\mathbf{g}_{n m}-\mathbf{g}_{n^{\prime} m^{\prime}}\right) \rho_{I}^{2}} e^{i\left(\mathbf{g}_{n m}-\mathbf{g}_{n^{\prime} m^{\prime}}\right) \mathbf{r}}
$$

Equation 3.59 was separated into two addends, the first one covering the partial waves, the second one the interference. Additionally, $\lambda_{i j}$ was divided according to $1 / \lambda_{i j}=\frac{1}{2}\left(1 / \lambda_{i}+1 / \lambda_{j}\right)$ [175]).

From equation 3.64 a few more or less obvious facts can be deduced:

- The electron intensity decreases exponentially with increasing $z$ and, in agreement with the normalization $P(z, \mathbf{r}) \cong 1$ for large $z$. Consequently, the implantation depth is limited to less than approx. one hundred nanometers, and the implantation energy is limited to less than 100-200 keV.

- There is an alternating behavior of the electron intensity depending on $z$, because of interferences between different states. In other words, there are more or less ideal implantation depths, which should be taken into account if the implantation energy can be freely varied. 11

- The emission channeling spectra, which are essentially a projection of the charge distribution, show s-, p- and d-type intensity patterns, although the latter ones are only observed

\footnotetext{
${ }^{11}$ In fact, this is less critical than the mean implantation depth. Although the implantation depth can be adjusted for a given axis, it will not be the optimum implantation depth for other axes whose measuring is necessary to unambiguously identify the lattice location of the emitter atoms.
} 
in cases where the emitter atoms are located beneath an atomic row rather than in the row [170].

\subsubsection{Experimental parameters}

Further modifications to equation 3.59 are necessary to account for experimental parameters, such as the implantation energy and the resolution of the setup. The first one is the depth distribution of the implanted emitter atoms, which is reasonably approximated by assuming a Gaussian depth distribution [176]. Therefore equation 3.59 needs to be folded with the implantation profile. The second modification is the smoothing of the simulated spectra with the angular resolution of the setup.

\subsubsection{Numerical details and extensions}

The simulation of theoretical emission channeling spectra can be done with the manybeam code - a FORTRAN77 based program, which was originally developed by Hofsäss [168]. Many of the subroutines within this program were taken from the CERNLib. All the emission channeling calculations performed within this work are computed using this package. A typical simulation of a $81 \times 81$ matrix takes less than one hour on a DEC Alpha $433 \mathrm{MHz}$ workstation. The input that is required to perform the simulations is mainly the two-dimensional unit cell in reciprocal Cartesian coordinates, the "atomic density", i.e. the mean distance between atoms in the $\mathrm{z}$-direction, the one-dimensional vibration amplitude of lattice and emitter atoms, the potential of the lattice atoms given in the parameterization of Doyle and Turner, and $Z$ of lattice and emitter atoms. A comparison between the experimental and the simulated spectra is performed with a least-squares fit implemented by Wahl [177].

\section{Extensions to the manybeam software}

To calculate the movement of an electron in continuum approximation, the construction of a 2-dimensional unit cell perpendicular to the principal z-axis is necessary. Usually this is done more or less manually with the use of crystallographic software. To account for more complicated lattice structures, such as the $6 \mathrm{H}-$ and the $4 \mathrm{H}-\mathrm{SiC}$ polytypes, as well as for lowdimensional axes which may result in a large number of atoms within the 2-dimensional unit cell the simulations had to be extended. This was done by an extension to the manybeam software which is essentially a FORTRAN77 code generator that outputs all the necessary code for an arbitrary axis under investigation. An algorithm was implemented that transforms the 


\subsection{ELECTRON EMISSION CHANNELING}

three-dimensional unit cell into a two-dimensional one by applying a set of transformations to a vector in the three-dimensional unit cell, namely coordinate system transformation, rotation and projection.

This extension allowed the calculation of emission channeling spectra for impurities in unit cells with a large number of atoms. This was recently demonstrated in emission channeling studies ${ }^{111} \mathrm{In}$ in the antiferromagnetic semiconductor $\mathrm{Cr}_{2} \mathrm{O}_{3}$ [178], which contains 30 atoms in the hexagonal unit cell of the corundum-type [179].

\subsubsection{Lanthanide isotopes for emission channeling investigation}

A big advantage of the emission channeling technique is the large number of possible isotopes than can be used. Criteria for the usefulness are:

- A half-life of two days to three months: this criterion depends on the detectors used, especially the maximum counting rate. In the case of off-line experiments, where the samples have to be shipped, a half-life of more than one week is desirable.

- The transitions must be converted sufficiently. This requests $\gamma$-energies lower than approx. $300 \mathrm{keV}$. The typical electron conversion coefficients for useful transitions range from 0.05 to 0.5 .

- No positrons and conversion electrons at the same time: some transitions far away from stability decay both via electron capture and positron decay.

- Low Q-factor, which prevents the nucleus from recoil implantation.

In table 3.3 possible electron emission channeling isotopes are listed. For Ho and Sm no useful decay with a half-life of more than two days exists. In the table possible transitions are listed, together with their half-lives, typea of decay, Q-factora, main $\gamma$-energies, the respective targets at the on-line isotope separator ISOLDE (see chapter 4.4), and the experiments, in which they were used for emission channeling studies. These experiments always refer to the transition and not to the element, because the convention used by Wahl and coworkers is different from the one used here. The convention used in this work is that, although conversion electrons originate from the daughter isotope of the corresponding transition, annealing of the sample is performed when the radioactive nucleus is the parent isotope of the transition. Although it is reasonable to assume a lattice location independent of the used lanthanide ions, for other element transmutations such as ${ }^{111} \mathrm{In} \rightarrow{ }^{111} \mathrm{Cd}$, where $\mathrm{In}$ is usually trivalent but $\mathrm{Cd}$ divalent, this difference in valence matters. 


\begin{tabular}{|c|c|c|c|c|c|c|c|}
\hline $\mathrm{Ln}$ & transition $^{\mathrm{a}}$ & half-life ${ }^{1}$ & decay & $\mathrm{Q}^{\mathrm{b}, 1}$ & $\mathrm{E}(\gamma)^{\mathrm{c}, 1}$ & $\mathrm{~T}^{\mathrm{d}}$ & exp..$^{e}$ \\
\hline \multirow[t]{2}{*}{$\mathrm{Ce}$} & ${ }_{58}^{139} \mathrm{Ce} \rightarrow{ }_{57}^{139} \mathrm{La}$ & $137.64 \mathrm{~d}$ & $\mathrm{EC}$ & 278 & 166 & $\mathrm{Ta}$ & $\mathrm{c}-\mathrm{BN}[52]$ \\
\hline & ${ }_{58}^{141} \mathrm{Ce} \rightarrow{ }_{59}^{141} \mathrm{Pr}$ & $32.50 \mathrm{~d}$ & $\beta^{-}$ & 581 & 145 & $\mathrm{UC}_{2}$ & Diamond [180] \\
\hline $\operatorname{Pr}$ & ${ }_{59}^{143} \mathrm{Pr} \rightarrow{ }_{60}^{143} \mathrm{Nd}$ & $13.57 \mathrm{~d}$ & $\beta^{-}$ & 934 & - & $\mathrm{UC}_{2}$ & $\mathrm{GaN}[181$ \\
\hline $\mathrm{Nd}$ & ${ }_{60}^{147} \mathrm{Nd} \rightarrow{ }_{61}^{147} \mathrm{Pm}^{*}$ & $10.98 \mathrm{~d}$ & $\beta^{-}$ & 896 & 91 & $\mathrm{UC}_{2}$ & $\mathrm{GaN}[182$ \\
\hline $\mathrm{Pm}$ & ${ }_{61}^{149} \mathrm{Pm} \rightarrow{ }_{62}^{149} \mathrm{Sm}$ & $53.08 \mathrm{~h}$ & $\beta^{-}$ & 1071 & 22.5 & - & \\
\hline $\mathrm{Sm}$ & & $<2 \mathrm{~d}$ & & & & & \\
\hline $\mathrm{Eu}$ & ${ }_{63}^{147} \mathrm{Eu} \rightarrow{ }_{62}^{147} \mathrm{Sm}$ & $24.1 \mathrm{~d}$ & $\mathrm{EC}$ & 1722 & 121,197 & $\mathrm{Ta}$ & \\
\hline \multirow[t]{2}{*}{$\mathrm{Gd}$} & ${ }_{64}^{146} \mathrm{Gd} \rightarrow{ }_{63}^{146} \mathrm{Eu}^{*}$ & $48.27 \mathrm{~d}$ & $\mathrm{EC}$ & 1030 & 115,155 & $\mathrm{Ta}$ & \\
\hline & ${ }_{64}^{149} \mathrm{Gd} \rightarrow{ }_{63}^{149} \mathrm{Eu}^{*}$ & $9.28 \mathrm{~d}$ & $\mathrm{EC}$ & 1319 & $150,265, .$. & $\mathrm{Ta}$ & Diamond [180] \\
\hline $\mathrm{Tb}$ & ${ }_{65}^{155} \mathrm{~Tb} \rightarrow{ }_{64}^{155} \mathrm{Gd}^{*}$ & $5.32 \mathrm{~d}$ & $\mathrm{EC}$ & 821 & $105,180, .$. & $\mathrm{Ta}$ & \\
\hline Dy & ${ }_{66}^{159} \mathrm{Dy} \rightarrow{ }_{65}^{159} \mathrm{~Tb}$ & $144.4 \mathrm{~d}$ & $\mathrm{EC}$ & 366 & 58 & $\mathrm{Ta}$ & \\
\hline Ho & & $<2 \mathrm{~d}$ & & & & & \\
\hline $\mathrm{Er}$ & ${ }_{68}^{169} \mathrm{Er} \rightarrow{ }_{69}^{169} \mathrm{Tm}^{\mathrm{M}}$ & $9.40 \mathrm{~d}$ & $\beta^{-}$ & 351 & 8.4 & - & \\
\hline \multirow[t]{6}{*}{$\mathrm{Tm}$} & ${ }_{69}^{167} \mathrm{Tm} \rightarrow{ }_{68}^{167} \mathrm{Er}$ & $9.25 \mathrm{~d}$ & $\mathrm{EC}$ & 748 & 208 & $\mathrm{Ta}$ & InP [183] \\
\hline & & & & & & & $\mathrm{SrTiO}_{3}$ [184] \\
\hline & & & & & & & GaAs [185] \\
\hline & & & & & & & Si [186] $]^{*}$ \\
\hline & & & & & & & $3 \mathrm{C} / 4 \mathrm{H}-\mathrm{SiC}$ [187] \\
\hline & ${ }_{69}^{168} \mathrm{Tm} \rightarrow{ }_{68}^{168} \mathrm{Er}$ & $93.1 \mathrm{~d}$ & $\mathrm{EC}$ & 1679 & 80,184 & - & \\
\hline \multirow[t]{4}{*}{$\mathrm{Yb}$} & ${ }_{70}^{166} \mathrm{Yb} \rightarrow{ }_{69}^{166} \mathrm{Tm}$ & $56.7 \mathrm{~h}$ & $\mathrm{EC}$ & 304 & 82 & $\mathrm{Ta}$ & \\
\hline & ${ }_{70}^{169} \mathrm{Yb} \rightarrow{ }_{69}^{169} \mathrm{Tm}^{\mathrm{M}}$ & $32.03 \mathrm{~d}$ & $\mathrm{EC}$ & 909 & $177,197, .$. & $\mathrm{Ta}$ & AlN [50] \\
\hline & & & & & & & 6H-SiC [187] \\
\hline & ${ }_{70}^{175} \mathrm{Yb} \rightarrow{ }_{71}^{175} \mathrm{Lu}$ & $4.19 \mathrm{~d}$ & $\beta^{-}$ & 470 & $113,282, .$. & - & \\
\hline
\end{tabular}

${ }^{a}$ Daughter isotopes marked with an asterisk are unstable.

${ }^{\mathrm{b}} \mathrm{Q}$-factor in keV.

${ }^{c}$ Main $\gamma$-energies in $\mathrm{keV}$. The corresponding conversion electron energies are calculated by subtracting the binding energies of the electrons (see e.g. [188])

${ }^{d}$ Target material at ISOLDE if the isotope is available.

${ }^{\mathrm{e}}$ Lattice location studies applying the corresponding transitions to lanthanide doped semiconductors. For references marked with an asterisk one or more alternative references exist. Underlined system and corresponding references are part of this thesis.

${ }^{1}$ Rounded values.

${ }^{\mathrm{M}}$ Room-temperature Mössbauer transition.

Table 3.3: Lanthanide isotopes suitable for off-line emission channeling investigations. Elements with a half-live shorter than two days have not been considered. All values are taken from ref. [188] 


\subsection{ELECTRON EMISSION CHANNELING}

In the following a more detailed description of the isotopes used for the emission channeling studies in this work will be given. The isotopes are discussed along with the conversion electron energy spectrum of their daughter isotopes, as well as the decay chain encountered upon implantation at the on-line isotope separator ISOLDE. Characteristics of the implantation are addressed as well. In addition to the values reported in ref. [188], the electron conversion coefficients $\alpha$ are taken from ref. [189].

\section{${ }^{139} \mathbf{C e}$}

The isotope ${ }^{139} \mathrm{Ce}$ was obtained by using a Ta-target. The precursors were not determined in this case, and therefore the known decay (chain) is given as:

$$
{ }^{139} \mathrm{Ce} \underset{\mathrm{Q}_{\beta^{-}}=278}{\stackrel{\mathrm{t}_{1 / 2}=137.64 \mathrm{~d}}{\longrightarrow}}{ }^{139} \mathrm{La}
$$

Although the half-life of more than 137 days is rather long, the branching ratios show that almost $100 \%$ of the nuclei end up in the first excited $165.86 \mathrm{keV}$ state. The electron conversion factor $\alpha_{K}$ from this excited state to the ground state is known to be 0.215 . The decay-scheme together with the conversion electron spectrum of ${ }^{139} \mathrm{La}$ is shown in figure 3.2.5. The Q-factor of $278 \mathrm{keV}$ is very low and prevents recoil implantation of the nucleus.

\section{${ }^{141} \mathbf{C e}$}

The isotope ${ }^{141} \mathrm{Ce}$ is the only $\beta^{-}$-emitter used in this work. In contrast to the other isotopes, ${ }^{141} \mathrm{Ce}$ is obtained from a $\mathrm{UC}_{2}$-target. The precursor implanted in this case in known to be ${ }^{141} \mathrm{Cs}$, which decays according to the chain

$$
{ }^{141} \mathrm{Cs} \underset{\mathrm{Q}_{\mathrm{EC}}=5255}{\stackrel{\mathrm{t}_{1 / 2}=24.92 \mathrm{~s}}{\longrightarrow}}{ }^{141} \mathrm{Ba} \underset{\mathrm{Q}_{\mathrm{EC}}=3216}{\stackrel{\mathrm{t}_{1 / 2}=18.27 \mathrm{~m}}{\longrightarrow}}{ }^{141} \mathrm{La} \underset{\mathrm{Q}_{\mathrm{EC}}=2502}{\stackrel{\mathrm{t}_{1 / 2}=3.92 \mathrm{~h}}{\longrightarrow}}{ }^{141} \mathrm{Ce} \underset{\mathrm{Q}_{\mathrm{EC}}=580.7}{\stackrel{\mathrm{t}_{1 / 2}=32.501 \mathrm{~d}}{\longrightarrow}}{ }^{141} \mathrm{Pr} \text {. }
$$

The Q-factor of the first decay ${ }^{141} \mathrm{Cs} \rightarrow{ }^{141} \mathrm{Ba}$ is very high and leads to $\beta$-induced neutron emission in this case. Another characteristic of the implantation of ${ }^{141} \mathrm{Cs}$ is the immediate release of Cs directly after the proton pulses. This happens so fast, that the current measurements are falsified. In addition to the continuous $\beta^{-}$-spectrum, conversion electrons from the $145 \mathrm{keV} \rightarrow 0 \mathrm{keV}$ transition arise. The electron conversion factor $\alpha_{K}$ is reported to be approximately 0.38 .

\section{${ }^{149} \mathbf{G d}$}

The isotope ${ }^{149} \mathrm{Gd}$ was used for emission channeling studies for the first time. $\gamma$-spectroscopy 
showed that in this case the implanted precursors are mainly both ${ }^{149} \mathrm{Dy}$ and ${ }^{149} \mathrm{~Tb}$ and one gets the following decay chain:

$$
{ }^{149} \mathrm{Dy} \underset{\mathrm{Q}_{\mathrm{EC}}=3812}{\stackrel{\mathrm{t}_{1 / 2}=4.23 \mathrm{~m}}{\longrightarrow}}{ }^{149} \mathrm{~Tb} \underset{\mathrm{Q}_{\mathrm{EC}}=3636}{\stackrel{83.3 \%, \mathrm{t}_{1 / 2}=4.118 \mathrm{~h}}{\longrightarrow}}{ }^{149} \mathrm{Gd} \underset{\mathrm{Q}_{\mathrm{EC}}=1319}{\stackrel{\mathrm{t}_{1 / 2}=9.28 \mathrm{~d}}{\longrightarrow}}{ }^{149} \mathrm{Eu} \underset{\mathrm{Q}_{\mathrm{EC}}=692}{\stackrel{\mathrm{t}_{1 / 2}=93.1 \mathrm{~d}}{\longrightarrow}}{ }^{149} \mathrm{Sm}
$$

This decay chain is not only attractive for emission channeling investigations, but also for Mössbauer spectroscopy studies, which can be performed on the final decay ${ }^{149} \mathrm{Eu} \rightarrow{ }^{149} \mathrm{Sm}$. The decay scheme of ${ }^{149} \mathrm{Eu}$ is shown in figure 3.2.5 together with the conversion electron spectrum (see also Ref. [190]). The most intense electrons are the $101 \mathrm{keV} \mathrm{K}$-conversion electrons of the $149.73 \mathrm{keV} \rightarrow 0 \mathrm{keV}$ transition. The $\alpha_{K}$ electron conversion factor is reported to be 0.48 . A disadvantage of the decay chain is that $16.7 \%$ of the ${ }^{149} \mathrm{~Tb}$ nuclei decay via $\alpha$-decay to ${ }^{145} \mathrm{Eu}$. The following decay chain is:

$$
{ }^{149} \mathrm{~Tb} \underset{\mathrm{Q}_{\alpha}=4077.3}{\stackrel{16.7 \%, \mathrm{t}_{1 / 2}=4.118 \mathrm{~h}}{\longrightarrow}}{ }^{145} \mathrm{Eu} \underset{\mathrm{Q}_{\mathrm{EC}}=2660}{\stackrel{\mathrm{t}_{1 / 2}=5.93 \mathrm{~d}}{\longrightarrow}}{ }^{145} \mathrm{Sm} \underset{\mathrm{Q}_{\mathrm{EC}}=616.7}{\stackrel{\mathrm{t}_{1 / 2}=340 \mathrm{~d}}{\longrightarrow}}{ }^{145} \mathrm{Pm} \underset{\mathrm{Q}_{\mathrm{EC}}=163.2}{\stackrel{\mathrm{t}_{1 / 2}=17.7 \mathrm{y}}{\longrightarrow}}{ }^{145} \mathrm{Nd} .
$$

Although this alternative decay chain is no problem with regard to the emission channeling investigations, a severe disadvantage is the long half-life of ${ }^{145} \mathrm{Pm}$ of 17.7 years. This situation makes ${ }^{149} \mathrm{Gd}$ very impractical, because the implanted samples are radioactive for more than one hundred years. Another severe problem that was encountered is the contamination with lanthanide monoxides. The resolution of the mass separator is insufficient to separate e.g. ${ }^{149} \mathrm{Dy}$ and ${ }^{133} \mathrm{Ce}^{16} \mathrm{O}$. Therefore we are additionally confronted with the following decay chain

$$
{ }^{133} \mathrm{Ce} \underset{\mathrm{QEC}_{\mathrm{EC}}=2900}{\stackrel{\mathrm{t}_{1 / 2}=4.9 \mathrm{~h}}{\longrightarrow}}{ }^{133} \mathrm{La} \underset{\mathrm{QEC}_{\mathrm{EC}}=2230}{\stackrel{\mathrm{t}_{1 / 2}=3.912 \mathrm{~d}}{\longrightarrow}}{ }^{133} \mathrm{Ba} \underset{\mathrm{Q}_{\mathrm{EC}}=517.4}{\stackrel{\mathrm{t}_{1 / 2}=10.52 \mathrm{y}}{\longrightarrow}}{ }^{133} \mathrm{Cs} .
$$

Such monoxide contaminations always exist, even for the lanthanide isotopes with high masses. The fraction of monoxides may, for lanthanides in the middle of the series, reach values much larger than that of the isotope of interest. Depending on the preparation conditions of the tantalum target, there may be twenty times more monoxides than desired isotopes. This makes the implantation of lanthanide isotopes from the middle of the series sometimes impossible. In principle, this problem can be overcome to a certain extent by using laser ionization rather than surface ionization.

\section{${ }^{167} \mathbf{T m}$}

The isotope ${ }^{167} \mathrm{Tm}$ is the one which accounts for most experiments so far, as shown in table 3.3 . 


\subsection{ELECTRON EMISSION CHANNELING}

In this case the implanted precursor is ${ }^{167} \mathrm{Lu}$, leading to the following decay chain

$$
{ }^{167} \mathrm{Lu} \underset{\mathrm{QEC}_{\mathrm{EC}}=3130}{\stackrel{\mathrm{t}_{1 / 2}=51.5 \mathrm{~m}}{\longrightarrow}}{ }^{167} \mathrm{Yb} \underset{\mathrm{Q}_{\mathrm{EC}}=1954}{\stackrel{\mathrm{t}_{1 / 2}=17.5 \mathrm{~m}}{\longrightarrow}}{ }^{167} \mathrm{Tm} \underset{\mathrm{Q}_{\mathrm{EC}}=748.3}{\stackrel{\mathrm{t}_{1 / 2}=9.25 \mathrm{~d}}{\longrightarrow}}{ }^{167} \mathrm{Er}
$$

Both the ${ }^{167} \mathrm{Lu}$ and ${ }^{167} \mathrm{Yb}$ precursors decay quite fast to ${ }^{167} \mathrm{Tm}$. The decay of ${ }^{167} \mathrm{Tm}$ to ${ }^{167} \mathrm{Er}$ is shown in figure 3.2.5, together with the $\mathrm{K}$ - and L-conversion electron energy spectrum of the $207.8 \mathrm{keV} \rightarrow 0 \mathrm{keV}$ transition of ${ }^{167} \mathrm{Er}$. The electron conversion coefficient $\alpha_{K}$ of this transition is reported to be 0.5 .

\section{${ }^{169} \mathbf{Y b}$}

The mother isotope ${ }^{169} \mathrm{Yb}$ was one of the first lanthanide isotopes used for emission channeling investigations. The initial precursor that was obtained at ISOLDE is ${ }^{169} \mathrm{Lu}$. The decay chain is given as follows:

$$
{ }^{169} \mathrm{Lu} \underset{\mathrm{QEC}_{\mathrm{EC}}=2293}{\stackrel{\mathrm{t}_{1 / 2}=34.06 \mathrm{~h}}{\longrightarrow}}{ }^{169} \mathrm{Yb} \underset{\mathrm{Q}_{\mathrm{EC}}=909}{\stackrel{\mathrm{t}_{1 / 2}=32.026 \mathrm{~d}}{\longrightarrow}}{ }^{169} \mathrm{Tm}
$$

Generally, ${ }^{169} \mathrm{Yb}$ is less preferable than ${ }^{167} \mathrm{Tm}$, because of the high $\gamma$-energies found in the decay ${ }^{169} \mathrm{Lu} \rightarrow{ }^{169} \mathrm{Yb}$. This makes the samples difficult to handle directly after implantation. Nevertheless, the longer half-life makes it a preferable isotope for off-line investigations. The conversion electron energy spectrum of the decay ${ }^{169} \mathrm{Yb} \rightarrow{ }^{169} \mathrm{Tm}$ is shown in figure 3.2.5. High electron intensities are found for the case of the $\mathrm{K}_{\alpha}$ and $\mathrm{K}_{\beta}$ conversion electrons of the $379.27 \mathrm{keV} \rightarrow 316.15 \mathrm{keV}$, the $316.15 \mathrm{keV} \rightarrow 138.93 \mathrm{keV}$ and the $316.15 \mathrm{keV} \rightarrow 118.19 \mathrm{keV} \gamma-$ transitions, of which usually only the latter two are used for experiments. For these two transitions, the reported $\alpha_{K}$ electron conversion coefficients are 0.5 and 0.38 , respectively. 

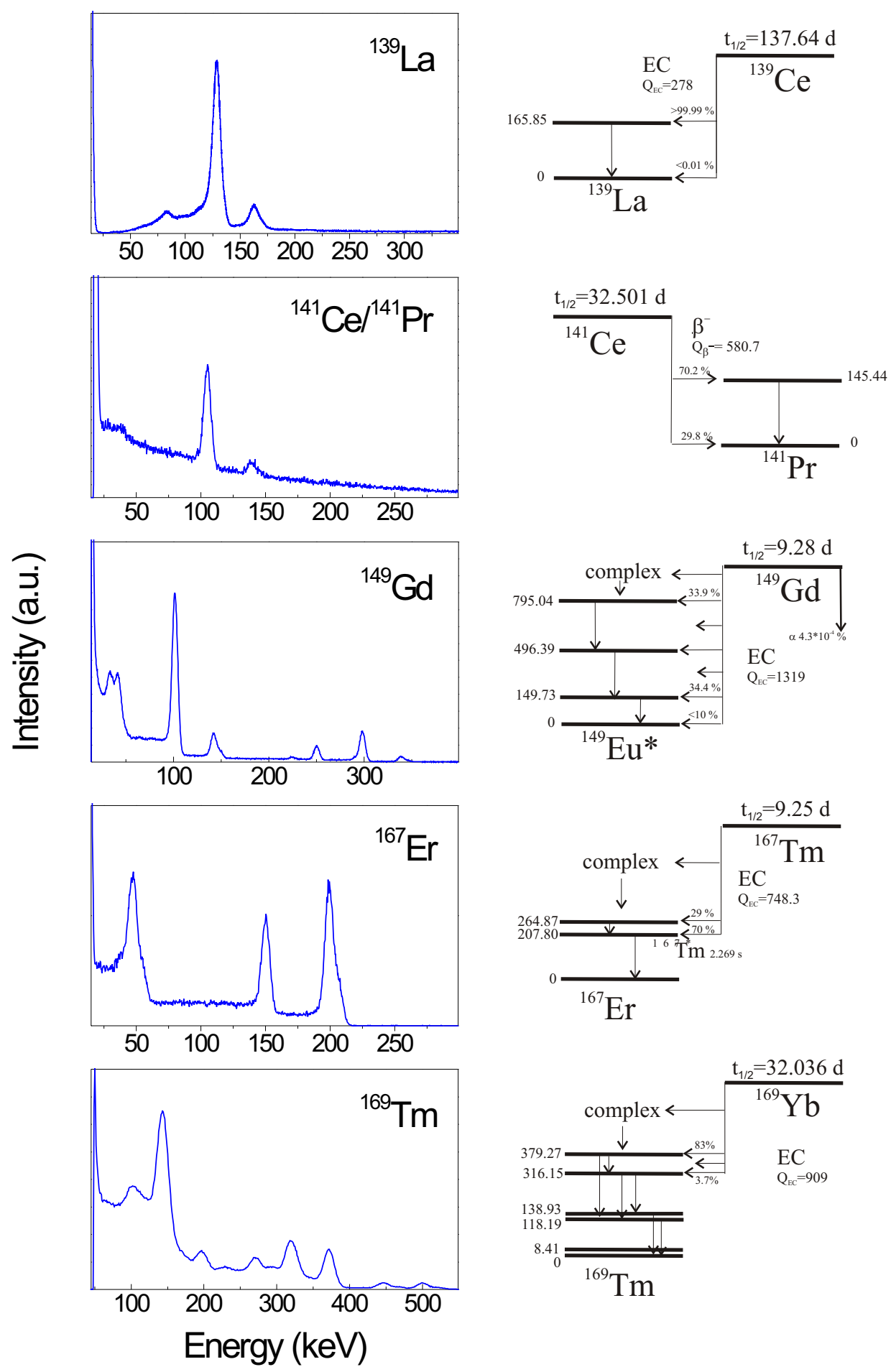

Figure 3.9: Electron energy spectra as well as simplified decay and level schemes of all lanthanide isotopes used for emission channeling experiments in this work. 


\subsection{Ion implantation}

Upon penetration of ions into a solid material (target), these ions are scattered at nuclei or at electrons in the solid. The cross sections for both processes depend on the energy of the incident ion, its mass and the mass of the target atoms. The second process, called electronic stopping, is dominant if high ion energies and small atomic numbers $Z$ are present. With decreasing kinetic energy of the ions or increasing atomic number $Z$, the first process, called nuclear stopping (at the Coulomb potential of the nuclei) becomes dominant [176]. Nuclear stopping leads to the generation of lattice damage if the energy transferred to the target atom is sufficient for a displacement. Both electronic stopping and nuclear stopping determine the implantation profile, i.e. the depth distribution of the implanted ions.

The implantation depth and corresponding straggling (equal to the variance of the distribution) can be calculated with the Monte-Carlo-simulation TRIM [191]. However, recent investigations of Palmetshofer et al. [192] revealed that for heavy ions the TRIM calculations may result in values that differ up to $20 \%$ from experimentally determined depth profiles. With regard to the accuracy of these parameters requested for emission channeling calculations, this difference is neglected. The concentrations of the implanted ions are not given separately. They may be calculated from mean implantation depth, variance and ion fluence. The fluences that are chosen for the implantation are all very low $\left(\leq 3 \cdot 10^{13}\right.$ ions $\left./ \mathrm{cm}^{2}\right)$; the concentration never exceeds $10^{19}$ ions $/ \mathrm{cm}^{3}$.

\subsection{Cathodoluminescence}

According to Mielenz [193], luminescence is the emission of optical radiation resulting from nonthermal excitation of the energy levels of atoms, molecules, polymers, and crystals. A further classification, including the excitation mechanism, can be given. Using cathode rays and electron beams as excitation sources, the resulting luminescence is called cathodoluminescence. Upon penetration of such electrons (often called primary electrons) into matter energy loss occurs via elastic or inelastic scattering at atoms, thereby generating secondary particles such as secondary electrons, Auger electrons, phonons, electron-hole pairs, X-rays and others [194]. The excitation of lanthanides is then due to inelastic scattering of primary or secondary particles at the $4 f$-electron system. The most famous application is the use of cathode rays in colour television tubes, where lanthanide doped phosphor transduces the kinetic energy of incident electrons into visible light (an overview is given by Ozawa [194]). In the case of lanthanide doped semiconductors, the application of cathodoluminescence is frequently encountered, 
especially for investigations of very wide band gap semiconductors, such as boron nitride or aluminum nitride (compare table A.3). This is due to the fact that cathodoluminescence setups are experimentally easier and cheaper to realize than photoluminescence setups, which require expensive lasers operating in the ultraviolet, or electrotroluminescence requiring the fabrication of a $p n$-junction $\sqrt{12}$ Given that sufficiently energetic electrons are used for excitations, usually with energies ranging from $100 \mathrm{eV}$ to about $30 \mathrm{keV}$, the energy needed for the excitation of an electron from the valence to the conduction band is provided. Barring this advantage, the main drawback in the application of cathodoluminescence for spectroscopic studies of lanthanide doped semiconductors is the large number of particles involved. Regarding the numerous ways $4 f$ electron systems can be excited (see chapter 2.1), the excitation conditions provided by electron beams are often not well defined. However, if the excitation processes are not a subject of investigation, $\mathrm{CL}$ provides an attractive experimental alternative.

Upon irradiation of a semiconductor energy carriers are created through which energy transfer to the lanthanide ion may occur, an inelastic process which may lead to an inner- $4 \mathrm{f}$ electron transition. Such carriers show a temperature-dependent lifetime, often decreasing with increasing temperature due to scattering at phonons and impurities. This is often expressed in terms of the mean lifetime $\langle\tau\rangle$ and a power law: $\langle\tau\rangle \propto T^{x}$ where $x$ depends on the scattering mechanism [125]. One may tentatively argue that the longer the lifetime of the carrier, the larger the probability that a lanthanide ion is hit, and this should be seen in temperature-dependent (td.) intensity measurements. In general, td. intensities are due to a superposition of many effects such as carrier lifetimes, td. lifetimes of the $4 f$ electron energy levels and td. excitation from e.g. an exciton to the $4 f$ electron system, which is possibly phonon-assisted.

In this work $\mathrm{CL}$ is used as the excitation mechanism in all the cases. However a theoretical description of the interaction of energetic electrons with matter cannot be justified in the scope of this thesis, because no electron energy-dependent measurements were performed. Calculations could be done using the Monte-Carlo code CASINO [196, 197, 198], but for the reasons discussed above this is omitted.

${ }^{12}$ An exception are the alternating current thin film electroluminescent (ACTFEL) devices, which are comparatively easy to realize [195]. 


\subsection{Mössbauer spectroscopy}

The Mössbauer effect, discovered in the late nineteen-fifties by R. Mössbauer [84], denotes the resonant absorption of the $\gamma$-radiation emitted by one nucleus by another nucleus of the same kind. Due to the recoil on the nuclei during emission and the absorption of a $\gamma$-quantum, resonance absorption may only occur if no momentum is transferred to the emitting and absorbing nuclei. This case may be realized by placing the atoms in a crystal, where, due to the large mass of the whole lattice, the momentum but almost no energy may be transferred to the lattice as a whole. The probability for such a recoilless emission is called Debye-Waller-factor and may be calculated using appropriate models.

Similar to the treatment of atoms, a nucleus system can be characterized using quantum numbers such as the angular momentum $I$, its z-component $M$ and parity $\pi$. Upon exposure to external magnetic or electric fields, the degeneracies of states characterized by the same quantum number $M$ are (partially) lifted, which becomes manifested in different energy eigenvalues with respect to the unperturbed system. Such shifts and splitting of levels may then be explored experimentally by altering the energy of the $\gamma$-radiation, applying what is known as the Doppler-shift, i.e. the change in frequency or wavelength of radiation due to movement with respect to the resting system. Moving the emitting nuclei (source) with respect to the resting nuclei (absorber), resonance absorption takes place when the Doppler-shift matches the necessary energy difference. Using a velocity drive and continuously passing through velocity ranges, characteristic Mössbauer spectra are recorded. These spectra reveal the type of interaction the nuclei are exposed to. The most important interactions are the isomer shift due to differing nuclear radii of, and s-electron density at, source and absorber nuclei, the quadrupole interaction due to the interaction with an electric field gradient, the magnetic dipole interaction with magnetic fields, and others. A complete description of the Hamiltonians and corresponding energy eigenvalues for these interactions can be found in Greenwood and Gibb [199]. Finally, it should be noted that evaluation of transition probabilities are performed in a similar manner as known from atomic physics, i.e. by calculating the matrix elements of multipole operators. 


\subsubsection{Lanthanide isotopes for Mössbauer spectroscopy investigation}

Similar to the lanthanide isotopes used for emission channeling investigations, a large number of appicable isotopes exist, which are suitable for Mössbauer spectroscopy studies. The only exception is the element cerium for which no suitable Mössbauer transition is known. However, if one is interested in those isotopes which feature a sufficiently large Debye-Waller factor (at least for room temperature investigations), the number of applicable isotopes is restricted to ${ }^{149} \mathrm{Sm},{ }^{151} \mathrm{Eu},{ }^{161} \mathrm{Dy}$ and ${ }^{169} \mathrm{Tm}$. In the following table the most important properties of these isotopes are summarized. As indicated in the table, only the neutron-deficient isotopes are sufficiently available at ISOLDE. The classification regarding possible electric or magnetic investigations was done by Stewart [91].

In general, not all the isotopes mentioned are suitable for the determination of the charge state or the electric field gradient. The isotope best suited for the determination of the charge state is ${ }^{151} \mathrm{Eu}$, because it features the largest isomer shift of approx. $10 \mathrm{~mm} / \mathrm{s}$ upon removal or addition of a $4 f$-electron $\left(\mathrm{Eu}^{3+} \rightarrow \mathrm{Eu}^{2+}\right)$. In contrast to this, the quadrupole splitting is in the range of the natural linewidth, which makes a determination of the electric field gradient difficult. The isotope of choice in this case is ${ }^{169} \mathrm{Tm}$, but the $8.4 \mathrm{keV}$ Mössbauer radiation is masked by lowenergy $\gamma$-radiation, which makes the use of high-resolution spectrometers obligatory. A feasibility study for ${ }^{169} \mathrm{Tm}$ implanted in GaN is shown in figure 3.10 . The spectrum was recorded

\begin{tabular}{c|cccc}
\hline & ${ }^{149} \mathrm{Sm}$ & ${ }^{151} \mathrm{Eu}$ & ${ }^{161} \mathrm{Dy}$ & ${ }^{169} \mathrm{Tm}$ \\
\hline $\mathrm{E}_{\gamma}(\mathrm{keV})$ & 22.5 & 21.6 & 25.65 & 8.4 \\
$\begin{array}{c}\text { Mother isotopes } \\
\text { (half-lifes) }\end{array}$ & ${ }^{149} \mathrm{Eu}(93.1 \mathrm{~d})$ & ${ }^{151} \mathrm{Sm}^{\mathrm{a}}(90 \mathrm{y})$ & ${ }^{161} \mathrm{~Tb}^{\mathrm{a}}(6.9 \mathrm{~d})$ & ${ }^{169} \mathrm{Er}^{\mathrm{a}}(6.9 \mathrm{~d})$ \\
$\alpha^{\mathrm{b}}$ & 12 & ${ }^{151} \mathrm{Gd}(120 \mathrm{~d})$ & ${ }^{161} \mathrm{Ho}(2.48 \mathrm{~h})$ & ${ }^{169} \mathrm{Yb}(32.0 \mathrm{~d})$ \\
$\mathrm{a}^{\mathrm{c}}(\%)$ & 13.9 & 52 & 5 & $\sim 300$ \\
Transition $\mathrm{I}_{g}(\mathrm{~L}) \mathrm{I}_{e}$ & $7 / 2-(\mathrm{M} 1) 5 / 2-$ & $5 / 2+(\mathrm{M} 1) 7 / 2+$ & $5 / 2+(\mathrm{E} 1) 7 / 2-$ & $1 / 2+(\mathrm{M} 1) 3 / 2+$ \\
$\mathrm{Q}_{g} / \mathrm{Q}_{e}{ }^{\mathrm{d}}$ & $+0.075 /+1.01$ & $+1.16 /+1.51$ & $+1.35 /+1.36$ & $0 /-1.3$ \\
$\mathrm{~W}_{0}{ }^{\mathrm{e}}$ & 1.71 & 1.30 & 0.38 & 8.33 \\
$\delta S^{\mathrm{f}}$ & -0.9 & -14.6 & +7.0 & -0.9 \\
Application $^{\mathrm{g}}$ & $\mathrm{QM}$ & $\mathrm{SM}$ & $\mathrm{QM}$ & $\mathrm{QM}$ \\
\hline
\end{tabular}

${ }^{\text {a }}$ Parent isotopes not available at ISOLDE.

${ }^{\mathrm{b}}$ Electron conversion coefficient.

${ }^{\mathrm{c}}$ Isotopic abundance.

${ }^{\mathrm{d}}$ Quadrupole moment.

e Natural line width $(\mathrm{mm} / \mathrm{s})$ [91].

${ }^{\mathrm{f}}$ Isomer shift (mm/s) per removal of one $4 \mathrm{f}$-electron [91].

g $\mathrm{S}=$ isomer shift, $\mathrm{Q}=$ electric quadrupole interaction, $\mathrm{M}=$ magnetic interaction [91].

Table 3.4: Lanthanide isotopes suitable for Mössbauer spectroscopy studies at room temperature. The values are taken from refs. [91, 199, 189]. 


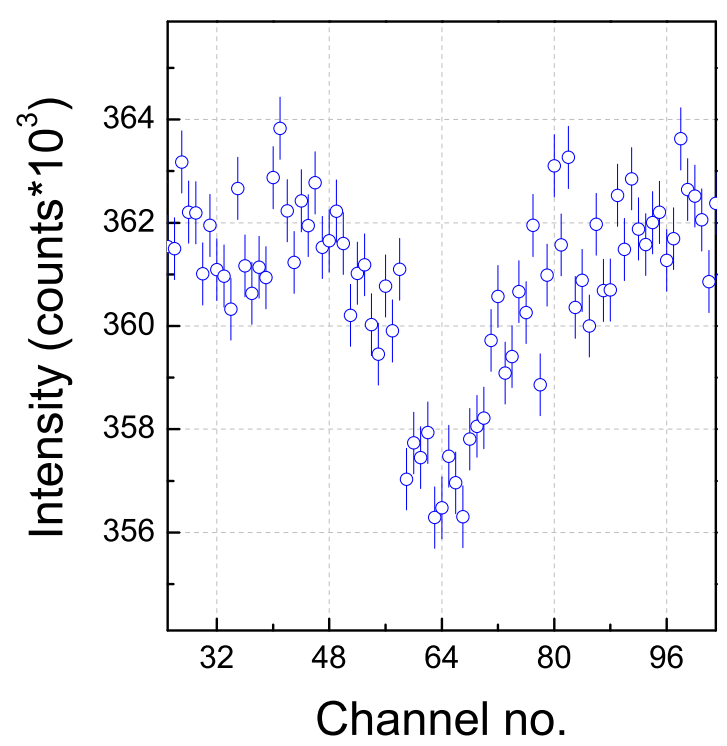

Figure 3.10: Transmission Mössbauer spectrum of ${ }^{149} \mathrm{Sm}$ in $\mathrm{GaN}$ recorded at room temperature. Absorber: $\mathrm{Sm}_{2} \mathrm{O}_{3}$ (not enriched), $100 \mathrm{~g} / \mathrm{cm}^{2}$.

using the setup described in chapter 4.3 , using a $\mathrm{Sm}_{2} \mathrm{O}_{2}$ powder absorber. The spectrum was recorded over a period of two months.

The corresponding $\gamma$-ray energy spectrum is shown in figure 3.11, together with the low en-
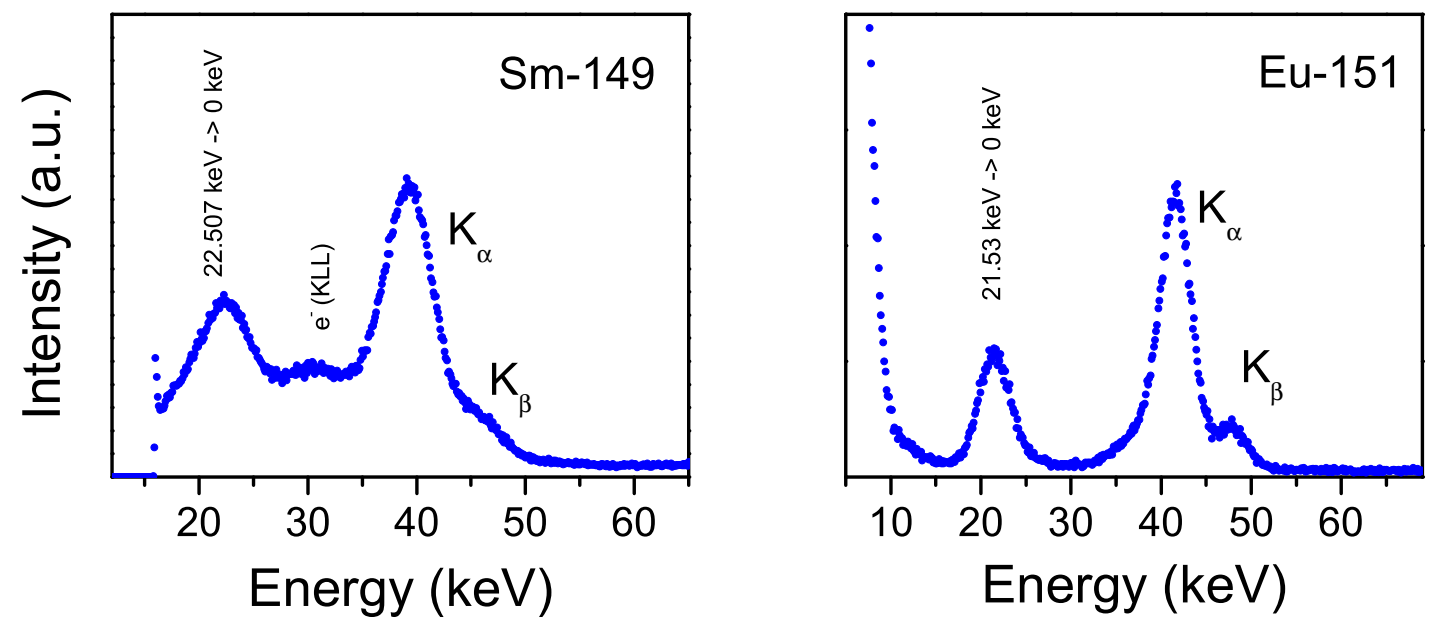

Figure 3.11: Low-energy $\gamma$-spectrum of ${ }^{149} \mathrm{Sm}$ and ${ }^{151} \mathrm{Eu}$, recorded with a Deetee silicon diode. Both energy spectra were recorded in air; in the case of the ${ }^{151}$ Eu energy spectrum a thin aluminium foil was mounted in front of the detector. 
ergy $\gamma$-ray spectrum of ${ }^{151} \mathrm{Eu}$, arising in the decay ${ }^{151} \mathrm{Gd} \rightarrow{ }^{151} \mathrm{Eu}$, which was used for the thin film measurements described in chapter 6 Although the results are promising, new detector developments are necessary to improve statistics and reduce measurement times.

\subsubsection{Mössbauer isotopes for thin film investigations}

Regarding investigations of films that are only a few nanometers thick, it is obvious that the method of choice is conversion electron Mössbauer spectroscopy (CEMS). The usability of the isotopes for CEMS applications is determined to a large extent by the $\gamma$-radiation additionally emitted in the decay. For this reason the parent isotope ${ }^{169} \mathrm{Er}$ is preferable to ${ }^{169} \mathrm{Yb}$, because the decay of the latter is accompanied by high-level $\gamma$ radiation. Of course other parameters, such as the cross section of the resonance absorption with respect to the penetration depth of the conversion electrons, will also influence the usefulness of the isotope. A comparative study of ${ }^{169} \mathrm{Tm}$ and ${ }^{151} \mathrm{Eu}$ for CEMS applications was given by Shenoy et al. [200], indicating that ${ }^{151} \mathrm{Eu}$ is best suited for CEMS measurements. 


\section{Experimental setups}

\subsection{The luminescence spectrometer}

The main technical work performed for this thesis was the assembly of a setup that allows the measurement of photoluminescence, cathodoluminescence and ionoluminescence. A brief description of this setup will be given below, with a focus on the time-resolved cathodoluminescence. Most of the parts of this setup were commercially available, except the time-resolved cathodoluminescence unit, which was completely developed and is therefore described in detail.

Figure 4.1 shows a schematic drawing of the luminescence setup. The samples are mounted on the head of a helium closed cycle refrigerator located inside a vacuum chamber, at pressures of typically $10^{-7}$ mbar. The samples are irradiated either with a laser, an electron gun or an ion beam ${ }^{1}$. The luminescent light is collected by an achromatic pair of lenses and, after passing through an optional long pass filter, focused into the spectrograph, were it disperses. The dispersed light is then focused onto one of the exchangeable detectors located at two possible exit slits.

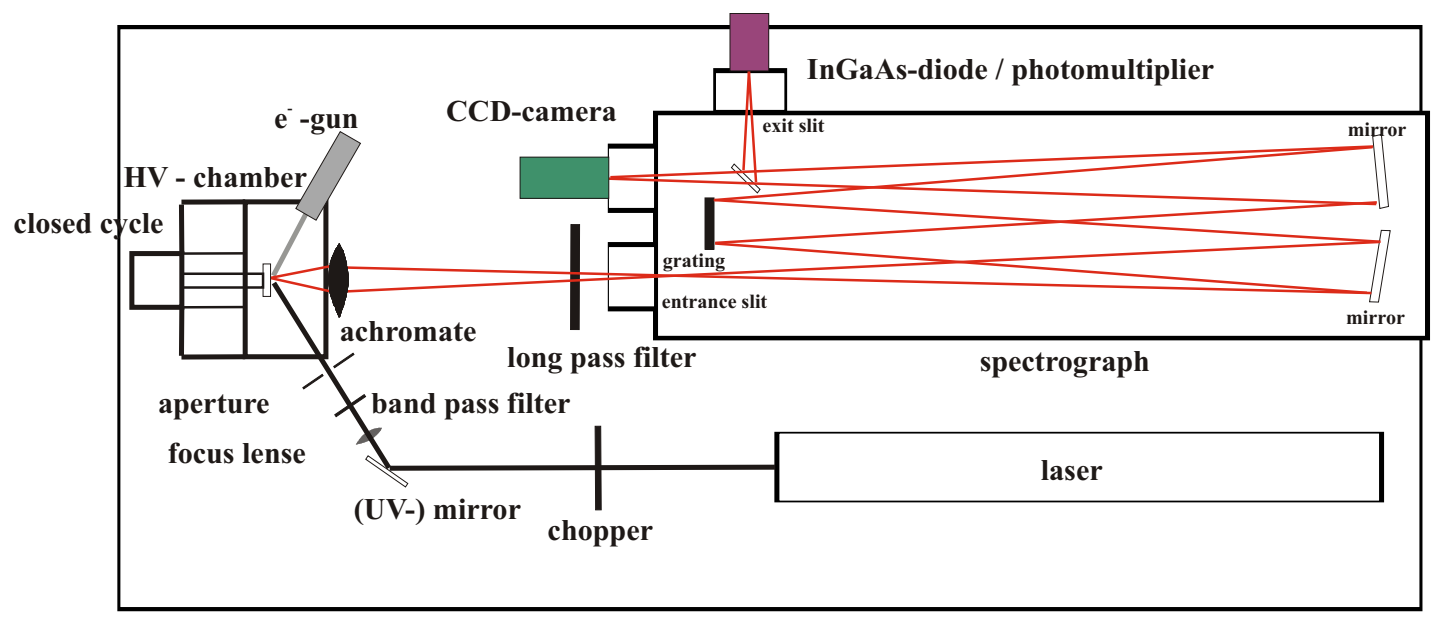

Figure 4.1: Schematic diagram of the luminescence setup.

\footnotetext{
${ }^{1}$ During the work on this thesis the setup was not attached to one of the accelerators.
} 


\subsubsection{Chamber, vacuum and cooling system}

The chamber itself is made of non-magnetic stainless steel and holds several sapphire windows that allow transmittance of light in the range of $180 \mathrm{~nm}$ to several micrometers. There are several additional flanges to attach the electron gun, the vacuum gauge, electrical feed-throughs, as well as a ventilation valve to. Most of the flanges are directed towards the sample. The standard incident angle for all beams is $60^{\circ}$ and may be changed using a modified sample holder. The windows are arranged in such a way that any incident excitation light leaves the chamber through another window, after being reflected at the sample. The chamber is anodized black to avoid reflection of stray light on the chamber walls.

The sample holder itself is made of oxygen-poor copper, and it allows the attachment of several samples, in an area of $1.5 \times 1.5 \mathrm{~cm}^{2}$. It also holds one of the two temperature sensors (silicon diode) on its top close to the samples. The sample holder is mounted on the head of a helium closed cycle refrigerator (CTI-Cryogenics model 22C/350C). A resistive heater is attached to the refrigerator head and controlled by means of an external temperature controller (LakeShore ${ }^{R}$ Model 331), allowing to adjust the temperature from $12 \mathrm{~K}$ to $300 \mathrm{~K}$ with an accuracy better than $0.1 \mathrm{~K}$. The sample holder as well as the refrigerator head are protected by an aluminum cooling shield, which is attached to the first cooling stage of the refrigerator and kept at a temperature of $77 \mathrm{~K}$. A pumping station is used to evacuate the chamber. Usual pressures are of the order of $10^{-7} \mathrm{mar}^{3}$

\subsubsection{Spectrograph and collection optics}

The luminescent light is collected with an achromatic pair of lenses 4 , consisting of two UVcoated convex and concave lenses, matching the image ratio of the spectrograph. The steradian is approximately $30^{\circ}$. Before passing the entrance slit of the spectrograph, the light passes through exchangeable optional long pass filters to suppress higher order luminescence. The spectrometer itself is a single grating Czerny-Turner spectrograph (Jobin Yvon 1000M) with a focal length of $1000 \mathrm{~mm}$, and is computer-controlled via a serial interface (see figure 4.2). After passing the manually adjustable entrance slit, the light is collected and parallelized with

${ }^{2} 12 \mathrm{~K}$ corresponds to the lowest stable temperature that may be achieved by slightly heating the sample. The lowest temporary temperature that can be reached is less than $10 \mathrm{~K}$.

${ }^{3}$ A pressure of $10^{-7}$ mbar was found to be critical for cathodoluminescence measurements, because condensing gas molecules, that are not trapped by the cooling shield, lead to a surface layer that may severely reduce the penetration depth of the electrons into the samples.

${ }^{4}$ The use of off-axis paraboloids would be more desirable and will be implemented in the future. 


\subsection{THE LUMINESCENCE SPECTROMETER}

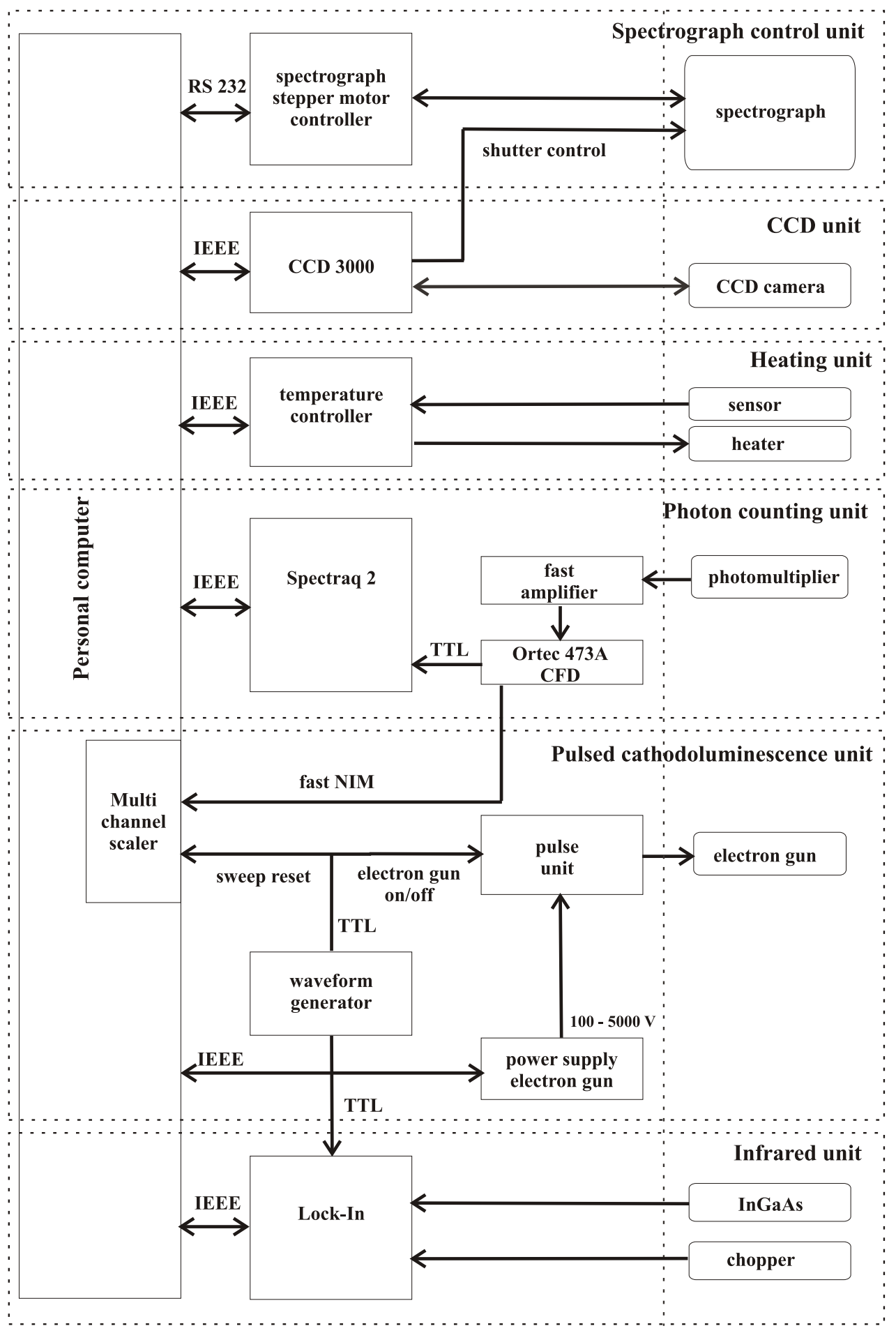

Figure 4.2: Diagram of the electronic part of the CL/PL setup. 
a toroidal mirror, before it is dispersed using easily exchangeable gratings 5 . Finally the light is collected by a second toroidal mirror and directed to one of the exit ports, which is selected via an electronically controlled beam director. At one of the exit ports, a CCD camera (see below) is permanently mounted, while at the second exit port, which features an additional mechanically adjustable slit, either a photomultiplier or an infrared detector is mounted. The maximal resolution for this setur 5 was determined to be $5 \mathrm{pm}$. Special attention was paid to the wavelength accuracy of the spectrograph. This was done by calibrating the spectrograph, monitoring the lines of a commercial mercury lamp (as described in ref. [201]) in first and second order. Deviations between measurements of the same lines after repeatedly moving the grating or between two well separated lines are found to be reasonably small and in the range of maximally $0.1 \mathrm{~nm} 7$. However, repeated re-calibration was necessary to allow for high resolution Stark level spectroscopy.

\subsubsection{Detectors}

- CCD camera The CCD camera (Yobin-Yvon Spectrum-One, liquid nitrogen cooled) is built from a UV-enhanced silicon charged coupled device with 1024 x 128 individual pixels (EEV, model CCD30-11, back illuminated, anti reflection coating, quantum efficiency: $>50 \%$ from $220 \mathrm{~nm}$ to $940 \mathrm{~nm}, 35 \%$ at $200 \mathrm{~nm}, 15 \%$ at $1000 \mathrm{~nm}$ ). The CCD is located in a vacuum sealed housing with a transparent entrance window. The detectable wavelength range covers $200 \mathrm{~nm}$ to more than $1000 \mathrm{~nm}$. Depending on the grating in the spectrograph a wavelength range between $20 \mathrm{~nm}$ and $240 \mathrm{~nm}$ can be monitored at once. The camera is controlled via an additional controller (Yobin-Yvon, model CCD 3000) which can itself be accessed by the computer via an IEEE ${ }^{8}$ interface. Using grating no. 1 and setting the entrance slit to $0 \mu \mathrm{m}$, a resolution of $40 \mathrm{pm}$ was obtained. As already mentioned, a above high wavelength accuracy is required for high resolution Stark level spectroscopy. A deviation of only $0.02 \mathrm{~nm}$ was measured between center and edge of the detector. It should be noted that, although a response function or the quantum efficiency of a CCD is in principle measurable, it is very time consuming if the wavelength range is repeatedly changed. Because of the long focal length of the spectrograph, survey spec-

\footnotetext{
${ }^{5}$ Three grating were available and used within this work. Grating 1: $12001 / \mathrm{mm}(300 \mathrm{~nm})$, grating 2: $6001 / \mathrm{mm}(700 \mathrm{~nm})$, grating 3: $100 \mathrm{l} / \mathrm{mm}(450 \mathrm{~nm})$. The value in brackets refers to the blaze wavelength.

${ }^{6}$ Using grating no. 1 , the photomultiplier and both slits were set to $0 \mu \mathrm{m}$

${ }^{7}$ A deviation of $0.1 \mathrm{~nm}$ is equal to $1.6 \mathrm{~cm}^{-1}$ at $800 \mathrm{~nm}$ and $11 \mathrm{~cm}^{-1}$ at $300 \mathrm{~nm}$.

${ }^{8}$ Institute of Electrical and Electronics Engineers
} 


\subsection{THE LUMINESCENCE SPECTROMETER}

tra could not be recorded in this manner ${ }^{9}$. However, the main disadvantage of the CCD camera is its rather low dynamic range $(<80,000)$. Therefore luminescence signals are difficult to observe if they are by more than two orders of magnitude lower in intensity compared to simultaneously detected signals ${ }^{10}$.

- Photomultiplier A conventional Hamamatsu R928 side window, uncooled photomultiplier is installed at one of the exit slits. Its quantum efficiency allows measurements in the wavelength range of $190 \mathrm{~nm}-750 \mathrm{~nm}$. The negative pulses at the anode are fed into a fast negative amplifier $\left(\mathrm{NIM}^{11}\right)$ before they are converted into positive $\mathrm{TTL}^{12}$ (500 ns pulse width) or fast negative NIM (25 ns pulse width) pulses, using a constant fraction discriminator (Ortec 473A, NIM). The TTL pulses are counted by an additional controller (Jobin Yvon, model Spectraq2), which is read out by the computer via an IEEE interface.

The main advantages of using a photomultiplier are that the spectra, which are in this case recorded in photon counting mode, can be easily corrected for the technical response function of the setup. Also time resolved luminescence spectroscopy is fairly easily realized, and the dynamic range is typically $>10^{6}$.

- Infrared Detector A low-cost InGaAs infrared detector (Electro Optical Systems) is available for measurements in the spectral range $800 \mathrm{~nm}-1700 \mathrm{~nm}$. The detector is attached to one of the exit ports using an adaptor which features an off-axis paraboloid for lightfocusing purposes. The signal can be fed either into an additional computer controlledcontroller with a built-in analog to digital converter (ADC) (current mode) or into a computer controlled lock-in amplifier (Stanford Research Systems, model SR830). If the measurements are performed in lock-in technique, a modulation of the excitation signal is achieved by either a chopper wheel (Stanford Research Systems, model SR540) or, in the case of infrared cathodoluminescence measurements, with the help of the time resolved CL unit. It has been found that the detector is insufficiently shielded from electronic noise. It was especially sensitive to the noise generated by the cooling system, which made measurements in current mode only feasible for very strong signals. An example spectrum is shown in figure 4.3 .

\footnotetext{
${ }^{9}$ Note that the so-called glueing, i.e. the merging of several spectra recorded at different wavelengths, gives rise to steps in the final spectrum because the grating is not turned continuously.

${ }^{10}$ Typical examples are Lanthanide Stark level spectra were the intensity ratios between two lines within the same multiplet to multiplet transition may reach values of $10^{4}$.

${ }^{11}$ Nuclear Instrument Module

${ }^{12}$ Transistor-Transistor Logic
} 


\subsubsection{Electron gun}

The electron gun is a Specs EQ 22 hot filament, constant beam electron gun, with an external controller, which is connected to the main computer via an IEEE interface. Its main features are variable electron energies in the range $100 \mathrm{eV}$ to $5 \mathrm{keV}$ and a variable electron beam current in the range $0.01 \mu \mathrm{A}$ to $250 \mu \mathrm{A}$. A beam sweep allows to sweep the electron beam across the sample, the electron beam is focused with a built-in electrostatic lens and a Wehnelt cylinder. The electron gun can be operated in either constant filament or constant electron beam current mode. Using this electron gun for CL measurements raises problems as regards the correction of the experimental spectra. The light emitted by the filament directly hits the sample, and consequently the experimental spectra are accompanied by this blackbody radiation. Although this represents by no means a drawback for high resolution CL measurements, spectra presenting an overview are not very reliable in case one requires precise values of intensities.

\subsubsection{Light sources}

Two constant wave (cw) laser light sources are currently available. One is a He-Cd laser (Topag, HCL-30 Ymc, $30 \mathrm{~mW}$ ) emitting light at $325 \mathrm{~nm}$, the other is a frequency doubled InGaAs diode emitting light at $535 \mathrm{~nm}$. Before the light enters the chamber through one of the entrance windows, it passes a short pass filter to suppress unwanted laser lines, and lenses for focusing purposes, and beam directors. The laser signal can be modulated using the chopper wheel mentioned above.

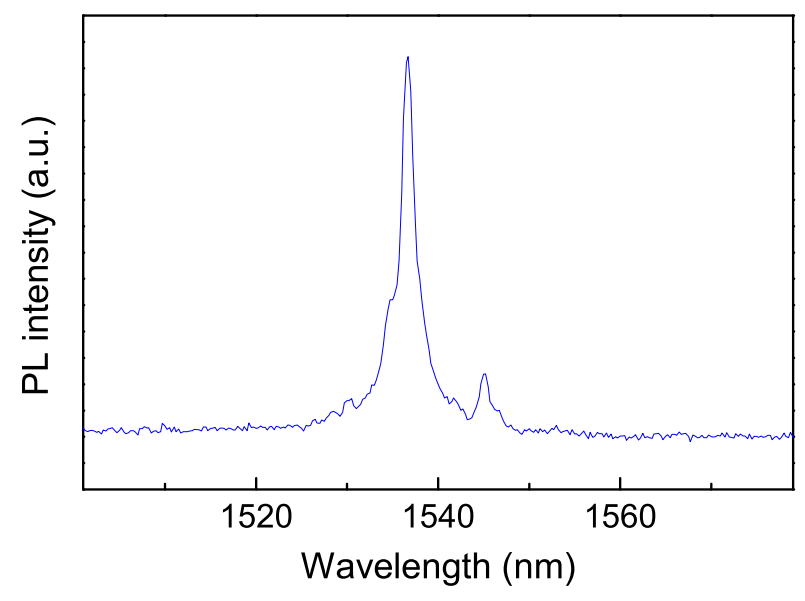

Figure 4.3: Example of infrared luminescence measurements. Shown is the luminescence arising from ${ }^{4} I_{13 / 2} \rightarrow{ }^{4} I_{15 / 2}$ electron transitions of $\mathrm{Er}^{3+}$ in $\mathrm{Si}$. The ions were implanted into Silicon at ISOLDE at an energy of $60 \mathrm{keV}$. The PL spectrum was recorded at $12 \mathrm{~K}$ under irradiation of the sample with laser light at $525 \mathrm{~nm}$. 


\subsection{THE LUMINESCENCE SPECTROMETER}

\subsubsection{Pulsed cathodoluminescence unit}

To allow for time-resolved CL spectroscopy the electron gun was modified in order to provide a pulsable electron beam. The approach was to repeatedly apply a defocusing voltage to the Wehnelt cylinder. To do so, the electrical connections between controller and electron gun were cut, as close to the electron gun as possible, in order to avoid any interferences from the capacitance of the wires. The voltage necessary to defocus the electron beam was provided by an external self-assembled electronic unit, whose circuit diagram is shown in figure 4.4. Its main task was to provide the defocusing voltage as well as to apply it to the wire connected to the Wehnelt cylinder (which was simply looped through the unit) if an external voltage is raised to a TTL trigger level. For this purpose an electric potential difference of $5 \mathrm{keV}$ has to be overcome. The pulse unit was triggered with a pulse generator (TTI, model TGP110) featuring

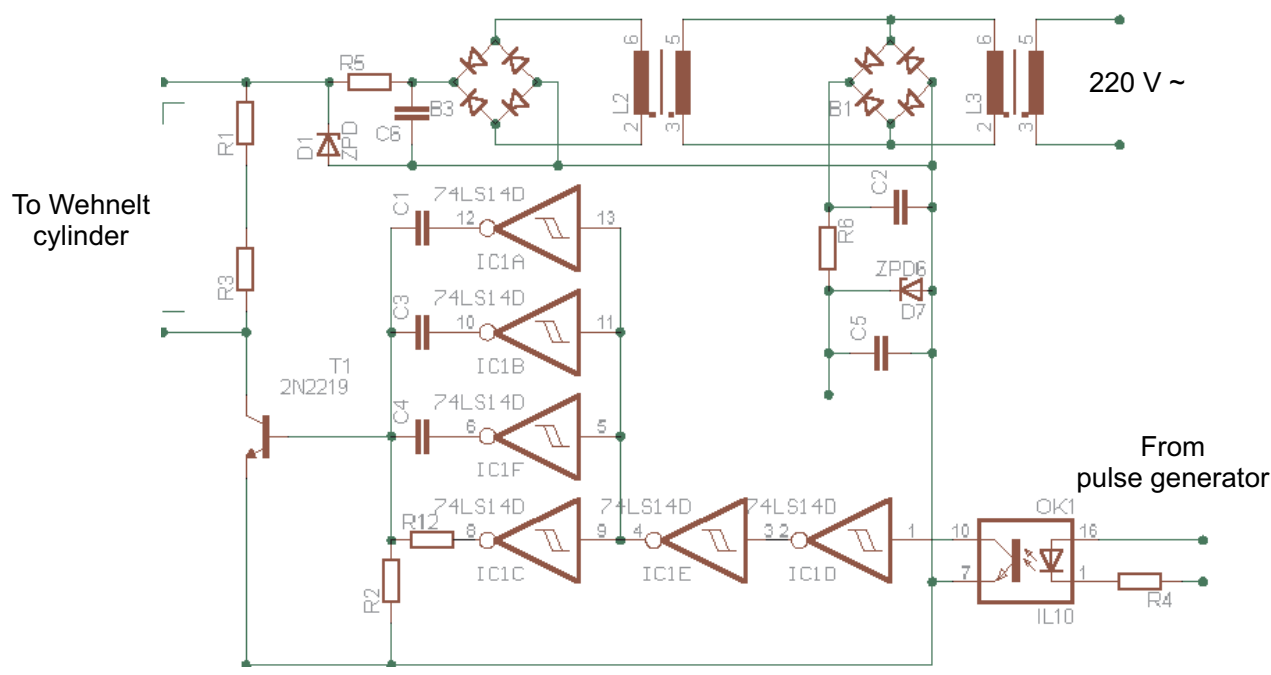

Figure 4.4: Circuit diagram of the pulse unit.

variable pulse length and frequency. The falling edge of the pulse unit was used to trigger a multi-channel scaler (MCS) unit (FAST ComTec, model 7882 dual input multiscaler/photon counter, ISA card) unit, while the fast NIM pulses (25 ns pulse width), that were generated by the constant fraction discriminator as a result of a photomultiplier signal, were counted by the MCS. The MCS features a minimum dwell timer of $125 \mathrm{~ns}$, channel advance was done through an internal clock. The signal pathway is shown in figure 4.2. To quantify the time resolution of this setup, a luminescence signal had to be found, which featured a fast decay compared to the dwell timer. ${ }^{13}$ Ideal candidates are the usually strong luminescence signals arising from the

${ }^{13}$ Using a prompt signal for the determination of the time resolution is frequently encountered in nuclear physics [202]. 

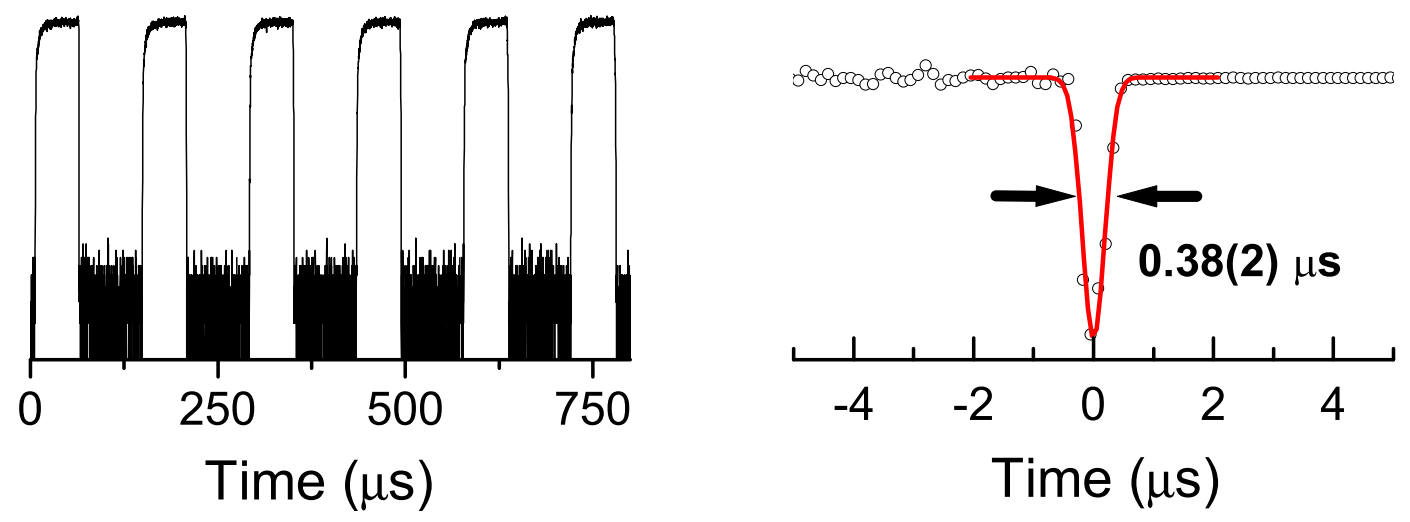

Figure 4.5: Left: electron gun on/off cycles (logarithmic) using GaN band edge luminescence. Right: time resolution of the setup measured at the derivative of the falling edge of the luminescence.

recombination of excitons bound to neutral donors or of free excitons in semiconductors. In this work GaN was chosen, because the lifetime of these donors at low temperatures is known to be less than $1 \mathrm{~ns}$ [203, 204, 205]. The decay signal and its first derivative are shown in figure 4.5. The first derivative can be fit to a Gaussian distribution with a FWHM ${ }^{14}$ of 380(20) ns. As the rise time of the voltage generated by the pulse unit was determined to $20 \mathrm{~ns}$ and typically a photomultiplier is characterized by an even better time resolution, the main reason for the only moderate time resolution must be attributed to the pulse generator. A resolution better than $20 \mathrm{~ns}$ is expected to be feasible, provided that an appropriate wave function generator and MCS are used. The best approach would be the use of microchannel-plate photomultipliers, allowing for a time-resolution of only a few ps.

Finally, it should be mentioned that another motivation to pulse the electron beam was to expand the wavelength range accessible for cathodoluminescence measurements towards the infrared region. Because in lock-in technique the exciting signal has to be modulated, this was found to be an attractive approach. Although this was tested and worked well, it was not applied to the samples discussed within this work.15

\footnotetext{
${ }^{14}$ Full Width at Half Maximum. The correlation between the FWHM and the variance $\sigma$ of a Gaussian distribution is $F W H M=\sigma \sqrt{8 \ln 2}$

15 This setup still does not allow time resolved spectroscopy in the infrared, simply because the InGaAs detector is too slow.
} 


\subsection{THE LUMINESCENCE SPECTROMETER}

\subsubsection{Correction of experimental spectra}

The wavelength dependent transmission and reflection properties of the individual components in the light path, such as lenses and gratings, as well as the quantum efficiencies of detectors require that the experimental spectra are corrected for this response function of the setup. The exact determination of such a response function is known to be very difficult because accurately calibrated light sources must be available, and the samples' properties must be taken into account [206]. Because detailed intensity calculations were not performed in this work, it was sufficient to determine the response function with an accuracy of about 10-20\%. As light source a commercially available halogen lamp (OSRAM Xenophot HLX) was used; its the intensity was approximated by a blackbody radiator with a temperature of around $3000 \mathrm{~K}$ [207]. The exact blackbody temperature depends on the operating conditions of the lamp, and needs to be determined separately using a pyrometer. The wavelength dependent intensity $I_{B B}$ is approximated by

$$
I_{B B}(T, \lambda)=I_{0}\left[\lambda^{5} \exp \left(\frac{1}{\lambda} \frac{h c}{k_{B} T}-1\right)\right]^{-1}
$$

and the response function is determined as the ratio of measured intensity and $\mathrm{I}_{B B}$.

In the paragraph describing the electron gun it was already mentioned that experimental CL spectra were accompanied by the radiation emitted by the filament because no optical beam dump existed. This radiation can be subtracted from the experimental spectra by assuming that the light emitted by the filament is similar to the one emitted by a blackbody radiator. However, this method is not very precise, wherefore most of the spectra, unless otherwise mentioned, were not corrected for the response function of the setup. Whenever the hint corrected for the response function of the setup is given for CL spectra in this work, the spectra are firstly corrected according to the description of the response function given above and secondly blackbody radiation is subtracted from the spectra (according to equation 4.1) when this was necessary.

In the case of high resolution Stark level spectroscopy it is necessary to correct the energetic positions of the individual lines for the refractive index of air in which the setup is operated. The first precise measurements of $n_{\text {air }}$ were performed by Edlén [208]. Later several groups modified Edlén's parametrization of $n_{\text {air }}$. In this work, the results of Boensch et al. [209] were used, who derived the following parametrization for the refractive index at T=293 K, p=100 kPa, and $0.04 \% \mathrm{CO}_{2}$ content: 


$$
\left(n_{\text {air }}-1\right)_{N} \cdot 10^{8}=A_{0}+B_{0} \cdot\left[B_{1}-\lambda^{2}\right]^{-1}+C_{0} \cdot\left[C_{1}-\lambda^{2}\right]^{-1}
$$

where $\lambda$ corresponds to the wavelength in $\mu \mathrm{m}, A_{0}=8091.37, B_{0}=2333983, B_{1}=130, C_{0}=15518$, and $C_{1}=38.9$. Regarding temperature, the condition $\mathrm{T}=293 \mathrm{~K}$ was fulfilled while performing experiments for this work. The possible influence of the pressure and $\mathrm{CO}_{2}$ content on the refractive index [209] is neglected. It is worth mentioning that Boensch et al. derived this formula for the visible part of the spectrum. Therefore equation 4.2 was applied to air wavelength - vacuum wave number pairs, listed in a variety of publications dealing with lanthanide Stark level spectra, showing consistency with equation 4.2, even for ultraviolet data sets. Unfortunately, the mentioned publications do not give references to the parameterization applied for $n_{\text {air }}$. 


\subsection{The emission channeling setup}

Figure (4.6) shows the principle of a typical emission channeling setup. The radioactive sample is mounted onto a three-axis goniometer. Under vacuum conditions, the electrons emitted from the sample are detected by a spatially fixed electron detector, which is either a diode, an array of diodes, or a CCD. For most of the emission channeling experiments performed for this work a surface barrier silicon diode was used as electron detector. To obtain one- or two-dimensional spectra, the goniometer has to be tilted repeatedly with a fixed step size of usually $0.1^{\circ}$ to $0.5^{\circ}$. The angular resolution of the setup is fixed via apertures on the samples and in front of the detector, and lies in the range $0.2^{\circ} \leq \sigma \leq 0.5^{\circ}$. For each measurement, the time necessary for the goniometer to remain at certain angular positions, was calculated. This time was estimated from the desired electron flux, compared with the current activity of the sample, and corrected taking the decay constant into account. The resulting spectra visualize the electron intensity with respect to the tilt angle between the sample's principal axis and the connection line between sample and detector.

A radiation heater allows to anneal the sample in situ at temperatures up to $1273 \mathrm{~K}$. This is very useful, as it allows to study the dependence of the lattice location of the implanted impurities on the annealing temperature (see e.g. Refs. [211] and [212]), without dismounting the samples from the goniometer.

Up to now, there exists only one emission channeling layout using two-dimensional electron detectors [213], which is installed at CERN. It allows to perform a raster scan of $(22 \times 22)$ without moving the goniometer. The advantage of this kind of setup lies in the fact, that an activity two orders of magnitude lower than that of the setup described above is needed, while the implantation fluence is maintained. This setup was used for one experiment in this work(see

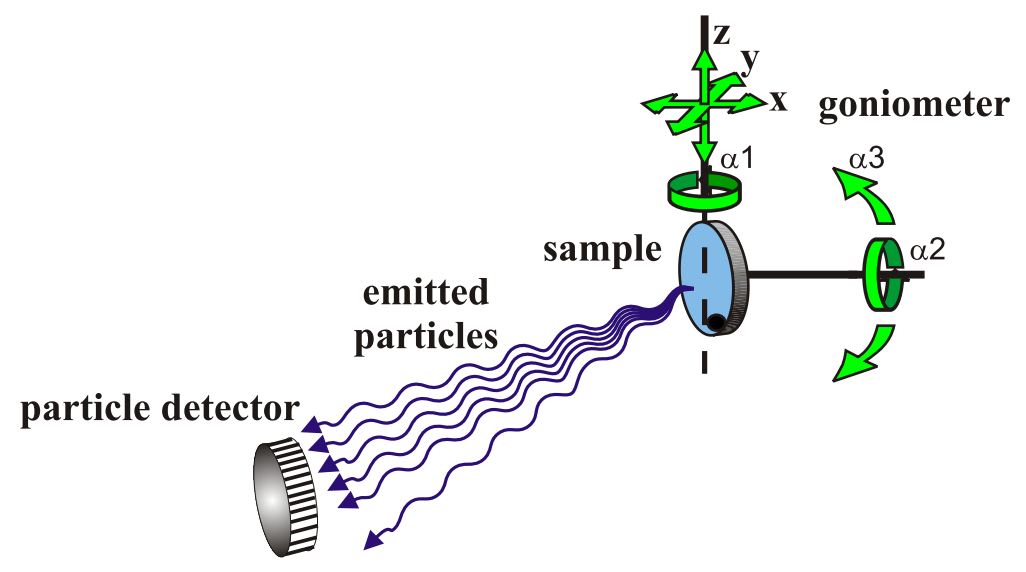

Figure 4.6: Principle of an emission channeling setup [210]. The radioactive sample, mounted on a three-axis goniometer, emits electrons which are detected by a spatially fixed electron detector. The heater allows in situ annealing of the sample under vacuum conditions. 
chapter 5.2.1]. It is worth mentioning that recently it has been shown by Hofsäss et al. [214] that charge-coupled devices, originally designed for X-ray spectroscopy in satellites, are very promising candidates for future EC layouts. 


\subsection{THE MÖSSBAUER SPECTROSCOPY SETUP}

\subsection{The Mössbauer spectroscopy setup}

For room-temperature Mössbauer spectroscopy a conventional setup using commercially available parts was employed. The development focused on the detectors as described below. A conventional drive (WiSSEl, model 260) was used to move the source; the drive was controlled with an external controller that allows to switch between differerent velocity shapes. The pulses from the detector were energy selected, converted into TTL pulses using a single channel analyzer and counted using a multi channel scaler. Two sources were available: a ${ }^{57} \mathrm{Fe}$ source was used for calibration purposes and a ${ }^{151} \mathrm{Sm}$ source $(350 \mathrm{mCi}$, kindly supplied by $\mathrm{H}$. Spiering, University of Mainz) was used for the thin film experiments in this work.

\subsubsection{Deetee detector}

For transmission Mössbauer experiments, as well as for electron emission channeling experiments, XRA- and XRB- silicon diodes $(500 \mu \mathrm{m})$ of the Finish company Deetee were used [215, 216]. These detectors feature a reasonable energy resolution at room temperature and a fair quantum efficiency for $\gamma$-radiation with an energy below $25 \mathrm{keV}$ (30\% for $22 \mathrm{keV} \gamma-$ radiation, according to the manufacturer's specification [215, 216]).

The Detectors were assembled as shown in figure 4.7. In order to use an available Ortec 142B preamplifier, the preamplifier's internal capacitance $C 2$ (see figure 4.7] [217] was bridged. A bias of $100 \mathrm{~V}$ was applied. The energy resolution $\Delta E / E$ (see figure 3.11) was measured to be 0.3 . This value could be increased significantly by cooling the detector. However, for the isotopes used in this work this was not necessary. The maximum counting rate without pile-up was determined to be $\sim 10 \mathrm{kHz}$.

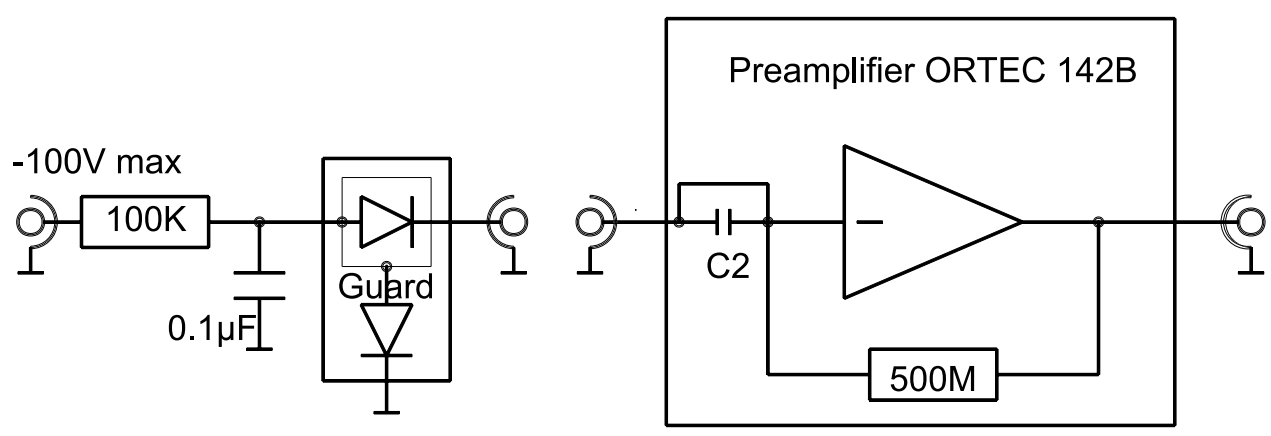

Figure 4.7: Wiring diagram of the deetee XRA and XRB detectors. The internal capacitance $\mathrm{C} 2$ of the Ortec 142B preamplifier is bridged. 


\subsubsection{CEMS detector}

To allow thin film conversion electron Mössbauer spectroscopy an appropriate detector had to be constructed. There are generally two designs that can be found in the literature. The first one is the parallel plate avalanche counter (PPAC), developed for on-line experiments, in which high counting rates may be required. This detector was developed by G. Weyer [218] and is operated using a $\mathrm{He} / \mathrm{CH}_{4}$ gas mixture or pure Aceton vapor at a pressure of approximately 150 mbar. The second CEMS detector design is a wire counter, which is a proportional counter operating with a $\mathrm{He}+10 \% \mathrm{CH}_{4}$ gas mixture (see e.g. [219, 220]). In contrast to the PPAC, this detector features a wire electrode and operates with a gas flow at normal pressure. This second design turned out to be more useful for thin film measurements, because in long-term measurements the conditions are more stable. The PPAC cannot be operated over a long period without refilling its gas reservoir, and a gas mixture is generally not advisable, because the individual detector gas components may separate. A comparative study for both detector designs can be found in Gancedo et al. [219].

In figure 4.8, an explosion drawing and the side view of the CEMS detector used in this work is shown. The sample was mounted on one of the plain electrodes (7), which was located inside the detector housing $(1,2)$. Opposite the sample, a wire electrode (4) was installed, and kept at a potential of around $+1000 \mathrm{~V}$. The detector was sealed with two Mylar ${ }^{R}$ foils glued to the outer parts of the detector housing (parts 1 and 5). The drill hole for wire and gas is also shown (part 2).
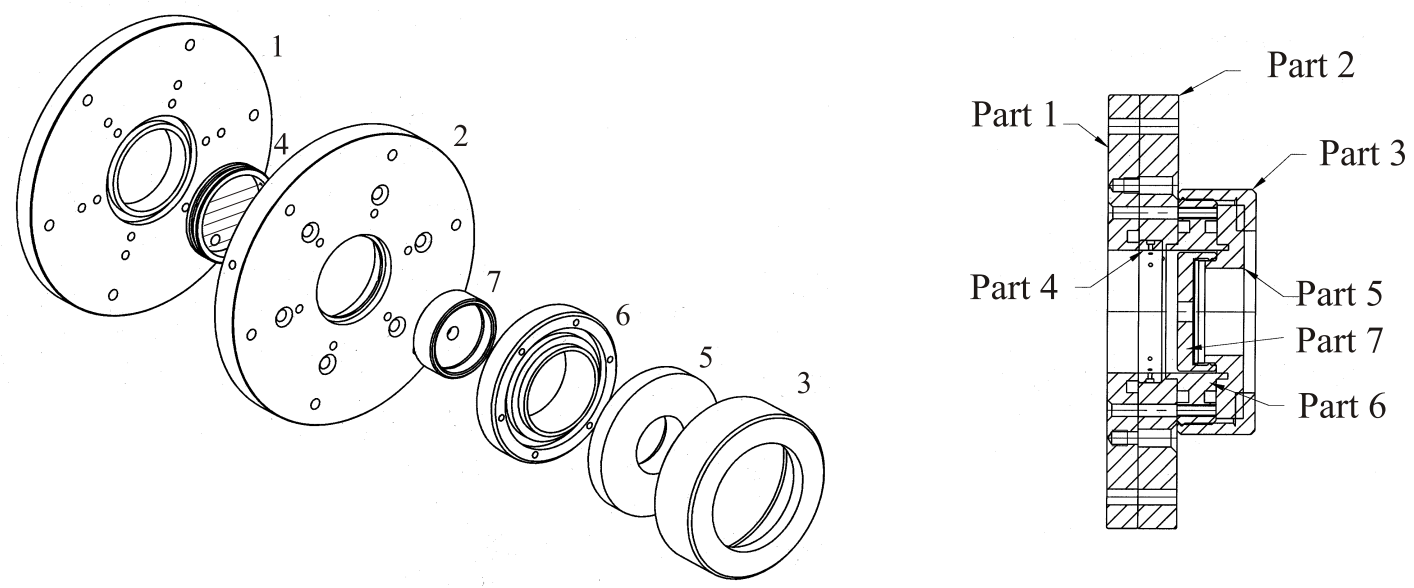

Figure 4.8: Explosion drawing and side view of the CEMS detector used in this work. 


\subsection{OTHER EXPERIMENTAL FACILITIES}

\subsection{Other experimental facilities}

In the following, other experimental facilities which were used in this work are briefly introduced. In contrast to the setups described before, no experimental developments at or modifications to these setups were necessary.

ISOLDE The on-line isotope separator ISOLDE is a large-scale experimental facility installed at the European center for nuclear research, CERN, in Geneva. Its main purpose is the production of radioactive nuclei, which are provided to experimental facilities installed in an experimental hall. The radioactive nuclei are produced in a fission reaction by bombardment of a target with protons of energies of $1.0 \mathrm{GeV}$ or $1.4 \mathrm{GeV}$. The radioactive atoms produced are extracted from the target, which is usually kept at a potential of $60 \mathrm{keV}$, by surface ionization or laser ionization. The energetic ions are then mass-separated and guided through long beamlines to implantation or other experimental facilities. This very complex system is fully computercontrolled; the transmission of ions through beamlines to the experimental facilities is very high $(>95 \%)$. An actual description of the ISOLDE facility can be found in ref. [221]. All the radioactive isotopes used in this work were implanted at ISOLDE and the samples shipped to Goettingen for off-line experiments, with the exception of the on-line experiments using ${ }^{57} \mathrm{Co}$.

ADONIS The experimental facility ADONIS ${ }^{16}$ [222, 223] is essentially a mass separator, used for the growth of thin films. The growth of these thin films using energetic ions is named mass separated ion beam deposition (MSIBD). The ions are produced in a Siddhenius-type ion source, in which either gases are used as raw materials, or solid materials are evaporated. After ionization, the ions are mass separated and deposited on substrates held in a special deposition chamber at pressures of $\sim 10^{-8}$ mbar. Deposition energies range from a few eV to several $\mathrm{keV}$. To allow film growth involving more than one ionic species, the mass separator can be switched between different masses by means of a computer. Following the deposition, the substrates can be transferred to an analysis facility without breaking the vacuum. At typical base pressures of $10^{-9}$ mbar photoelectron-spectroscopy (XPS,UPS), Auger electron spectroscopy (AES) and electron energy loss spectroscopy (EELS) facilities are provided. A helium gas discharge lamp and an $\mathrm{AlK}_{\alpha} / \mathrm{Mg}_{\alpha} \mathrm{x}$-ray source are the excitation sources. The analysis is performed with a cylindrical mirror analyzer (Perkin-Elmer/Physical Electronics, model PHI 15-555).

The obvious advantage of MSIBD thin film growth is that the ions are well known and isotope controlled, without any purity requirements on the raw materials. The main problem is that

\footnotetext{
16 Anlage zur DepOsition Niederenergetischer Ionen auf Substrate.
} 
the thin film growth is extremely slow (only a few tens of nanometers per hour), and for many elements the current may only reach a few nanoamperes. However, this problem is solely related to the ion source and will hopefully be overcome in the future.

IONAS The implantation facility IONAS ${ }^{17}$ [224, 225] is a mass separator with a maximal potential difference between ion source and substrate of $530 \mathrm{kV}$ (High Voltage). The ions are produced in a Siddhenius-type ion source and, after mass separation, guided to implantation and material characterization facilities. All samples doped with stable lanthanide isotopes in the scope of this thesis where implanted at IONAS.

${ }^{17}$ IONenAnSchubser 


\section{Lanthanide implanted wide band gap III-V nitrides and carbides}

\subsection{Aluminum nitride}

2H-aluminum nitride (AlN) with its high thermal conductivity and stability, chemical inertness, and band gap of more than $6 \mathrm{eV}[134]$ is a very promising candidate for the implementation of ultraviolet light emitters. Due to the wide band gap, the possible optical applications of rare earth based light emitters are extended, exploiting parts of the energy level diagram of the rare earths that are hidden to prominent hosts such as $\mathrm{GaN}$ or $\mathrm{Si}$ (cf. figure 3.2). Typical examples are the upper energy levels of $\mathrm{Tm}^{3+}[50]$ and $\mathrm{Gd}^{3+}$, but also rare earth elements at the beginning of the lanthanide series, namely $\mathrm{Ce}^{3+}$ and $\mathrm{Pr}^{3+}$, whose energy level structures predict the lowest $5 \mathrm{~d}$ levels at positions still within the band gap of the widest band gap semiconductors [226]. This makes such systems interesting candidates for phosphor illuminators, which play an important role in the design of white light emitters [55]. The potential of AlN as a host for light emitters based on radiative intra-4f electron transitions of triply ionized lanthanides has recently been demonstrated for e.g. $\mathrm{Er}^{3+}-$ [45, 46, 227, 47, 48, 49], $\mathrm{Eu}^{3+}-$ and $\mathrm{Tb}^{3+}-$ [43, 228] and $\mathrm{Tm}^{3+}[50]-$ doped AlN. The annealing behavior of ion implanted AlN using radioactive

${ }^{111}$ In as probe atoms has recently been given [229], showing that about $50 \%$ of the probe atoms are located on undisturbed hexagonal sites after annealing the samples at a temperature of at least $1073 \mathrm{~K}$. A comparative annealing study under $\mathrm{N}_{2}$ atmosphere of group III-V-nitrides including $2 \mathrm{H}-\mathrm{AlN}$ can be found in the work of Hong et al. [230].

Investigations of the luminescence properties of as-grown $2 \mathrm{H}-\mathrm{AlN}$ thin films were performed by several authors [231, 232, 233, 234, 235, 236, 237]. Most of these works report on band gap related luminescence, besides the typical violet broadband luminescence [234, 233] which is attributed to oxygen-related defects [238, 231, 239]. Raman studies of phonons of 2H-AlN are reported by several authors (see e.g. Refs. [240, 241, 242, 243]), an example of first-principle calculations of vibrational properties can be found in the work of Miwa and Fukumoto [244]. A comparison of the physical properties and technological application of $2 \mathrm{H}-\mathrm{AlN}$ and other III-V-nitrides can be found in the reviews of Strite and Morkoç [245], Morkoç et al. [246] and Ambacher [247].

The most important physical properties of $2 \mathrm{H}-\mathrm{AlN}$ with regard to this work are summarized in table A.3. The unit cell of $2 \mathrm{H}-\mathrm{AlN}$ is shown in Figure A.1. The lattice constants and positions of 
the $\mathrm{Al}$ and $\mathrm{N}$ atoms in the unit cell are input parameters for the emission channeling investigation reported in chapter 5.1.1.

\subsubsection{Lattice location of ${ }^{169} \mathrm{Yb}$ in AIN}

In order to perform lattice location studies of lanthanide implanted AlN, radioactive ${ }^{169} \mathrm{Lu}$ ions were implanted into AlN (grown by metal-organic vapor phase epitaxy on $6 \mathrm{H}-\mathrm{SiC}$, as described in ref. [248]) at an energy of $60 \mathrm{keV}$ and fluences of $1 \cdot 10^{13}$ ions $/ \mathrm{cm}^{2}$ at the online isotope separator ISOLDE at CERN. The chosen decay chain is well-known and was already applied to other hosts in the past.

After more or less complete decay of ${ }^{169} \mathrm{Lu}\left(\mathrm{t}_{1 / 2}=35 \mathrm{~h}\right)$ to ${ }^{169} \mathrm{Yb}\left(\mathrm{t}_{1 / 2}=32.0 \mathrm{~d}\right)$, electron emission channeling spectra were recorded using the conversion electrons arising in the decay ${ }^{169} \mathrm{Yb} \rightarrow{ }^{169} \mathrm{Tm}$. The conversion electron energy spectrum as well as the decay scheme are shown in figure 3.2.5. The measurements were performed with the setup described in chapter 4.2. The angular resolution of the setup was $\sigma=0.3^{\circ}$. Isochronal annealing (10 min.) at $1273 \mathrm{~K}$ using a radiation heater was performed without dismounting the sample from the goniometer, making use of the stability of AlN under vacuum annealing conditions as shown in ref. [229]. To identify the lattice location of the implanted ions, experimental EC channeling spectra were fitted against simulated spectra as described in chapter 3.2.4. Figure 5.1 shows the normalized 2-dimensional EC spectra of conversion electrons of ${ }^{169} \mathrm{Tm}$ in $2 \mathrm{H}-\mathrm{AlN}$ as implanted, for the c-axis as well as for the [1213]-axis. On the right-hand side, the simulated patterns are shown, calculated with the manybeam code. The best fitting results are obtained by assuming that 69 (5) \% of the emitter atoms are located on substitutional Al lattice sites with a mean static displacement of $0.24 \AA$. The remainder of the emitter atoms is assumed to be located on sites of low symmetry, so called "random" sites. Upon annealing of the sample at $1273 \mathrm{~K}$ the substitutional fraction slightly increased to 78 (5) \%. This behavior is similar to that of lanthanide implanted GaN, in which after implantation a large fraction of the implanted ions is located on substitutional Ga sites. This fraction stays nearly constant upon annealing [181, 21].

The result of the emission channeling experiment suggests that lanthanides implanted into $2 \mathrm{H}-$ AlN are located on cationic sites replacing an $\mathrm{Al}$ atom 1

This situation is shown in Figure 3.3 . Intuitively, one might assume a local symmetry $\mathrm{C}_{3 v}$, but with respect to the observation made by Gruber et al. that luminescence investigations of lanthanides implanted into GaN may show a symmetry lower than $\mathrm{C}_{3 v}$, one has to restrain

\footnotetext{
${ }^{1}$ At the time of writing this thesis, these measurements are being complemented by lattice location studies of ${ }^{147} \mathrm{Nd} /{ }^{147} \mathrm{Pm}$ for the annealing temperatures T=RT,873 K,1373 K [249], showing the same lattice site.
} 

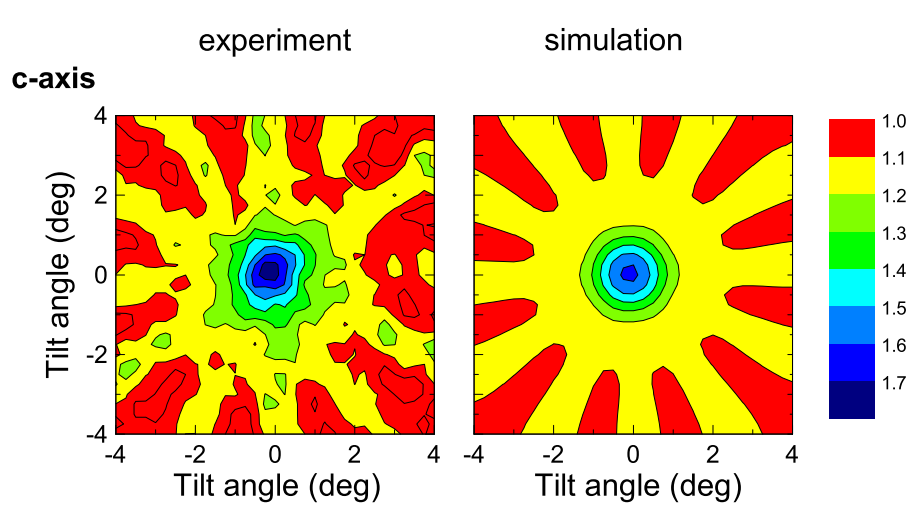

\section{[1213]-axis}
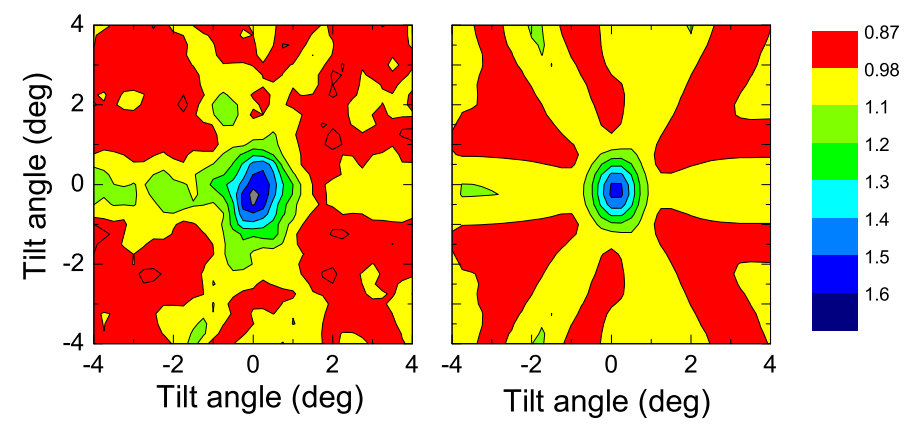

Figure 5.1: Left: normalized 2-dimensional channeling spectra of conversion electrons of ${ }^{169} \mathrm{Tm}$ in $2 \mathrm{H}$-AlN as implanted, for the c-axis as well as the [1213]-axis. Right: best fits to experimental spectra correspond to a fraction of 69(5) \% of emitter atoms on substitutional $\mathrm{Al}$ sites, with a mean static displacement of 0.24 . The remainder of the emitter atoms is assumed to be located on sites of low symmetry.

oneself from drawing such conclusions. Therefore, in addition to lattice location studies crystal field calculations were done in this work (see chapter7).

Primarily, the aim was to use those samples studied by emission channeling investigations for cathodoluminescence spectroscopy studies. This approach was alread used in ref. [50]. But as already pointed out in chapter 3.2.5, slight contaminations of lanthanide monoxide ions, which cannot be separated from the lanthanide ions with the same mass by the mass separator due to its limited resolution, are detected in the sample after implantation. Although these contaminations are small if the decay chain used for emission channeling investigations involves the isotope ${ }^{169} \mathrm{Yb}$, it is preferable not to use the samples implanted at ISOLDE for spectroscopic studies. In the case of the samples used for lattice location studies, besides the expected luminescence attributed to intra-4f electron transitions of $\mathrm{Tm}^{3+}$, contaminations involving $\mathrm{Gd}^{3+}$ ions as well as $\mathrm{Eu}^{3+}$ ions could be identified based on the measurements of AIN samples implanted with the 
corresponding stable ions, which will be discussed in the following chapters. As a result of this experience, all spectrosopic studies were performed at samples not implanted with radioactive ions.

\subsection{2 $\mathbf{G d}^{3+}$ doped AlN - an attractive $318 \mathrm{~nm}$ UV light emitter}

From the Dieke diagram shown in figure 3.2 it is obvious that the energy level scheme of $\mathrm{Gd}^{3+}$ differs from the other lanthanides in that the first excited state ${ }^{6} P_{7 / 2}$ is by far the one most separated from the ground state among the whole series. The energetic position above the ground state ${ }^{8} S_{7 / 2}$ is, according to the mean free ion parameters of $\mathrm{Gd}^{3+}$, predicted at $32168 \mathrm{~cm}^{-1}$. Apparently, $\mathrm{Gd}^{3+}$ should be an attractive ultraviolet light emitter, but as already pointed out in chapter 3.1.8, a reasonable transition probability between excited states below 50,000 $\mathrm{cm}^{-1}$ and the ground state, necessary for ultraviolet light emission, is most likely for the first excited level ${ }^{6} P_{7 / 2}$. A description of the most important properties of $\mathrm{Gd}^{3+}$ and useful references to spectroscopic studies were given in chapter 3.1.8. It is amazing that up to now $\mathrm{Gd}^{3+}$ has not been investigated in wide band gap semiconductors, although a reasonable number of hosts (e.g. AlN and $\mathrm{BN}$ polytypes) featuring the appropriate band gap is available.

In order to perform cathodoluminescence studies on $\mathrm{Gd}^{3+}$ implanted $\mathrm{AlN}$, stable ${ }^{158} \mathrm{Gd}$ ions were implanted at the IONAS implantation facility, as described in chapter 29, at an energy of $100 \mathrm{keV}$ and several fluences of up to $3 \cdot 10^{13} \mathrm{ions} / \mathrm{cm}^{2}$. Because some of the luminescence related to $\mathrm{Gd}^{3+}$ was very weak, in the following the discussion will be restricted to the samples implanted with the highest fluence of $3 \cdot 10^{13}$ ions $/ \mathrm{cm}^{2}$. Following implantation, the samples were annealed under vacuum conditions $\left(\sim 10^{-6}\right.$ mbar $)$ at $1373 \mathrm{~K}$ for 30 minutes. Cathodoluminescence measurements in the temperature range of $12 \mathrm{~K}$ to $300 \mathrm{~K}$ were performed using the setup described in chapter 4.1. The excitation conditions were, unless otherwise indicated, fixed to electron energies of $5 \mathrm{keV}$ and a power density of $0.3 \mathrm{~W} / \mathrm{cm}^{2}$.

Figure 5.2 shows the survey scan, recorded at room temperature as well as $12 \mathrm{~K}$. Besides the oxygen-related defect luminescence [238] of the AlN host, a strong and sharp line centered at $318 \mathrm{~nm}$, accompanied by two weaker lines, is visible. This luminescence is attributed to $\mathrm{Gd}^{3+}$, and obviously the strong line is expected to originate from radiative transitions between the first excited state ${ }^{6} P_{7 / 2}$ and the ground state of $\mathrm{Gd}^{3+}$. Besides two weaker lines accompanying the ${ }^{6} P_{7 / 2} \rightarrow{ }^{8} S_{7 / 2}$ transition, no further transitions are detected in the survey scan. This is remarkable as one may, based on the energy level diagram of $\mathrm{Gd}^{3+}$ and transition probabilities, expect transitions from the ${ }^{6} G_{7 / 2}$ level, as its energetic position is still within the band gap of the AlN host. These transitions should give rise to a reasonable luminescence in the visible part of the 


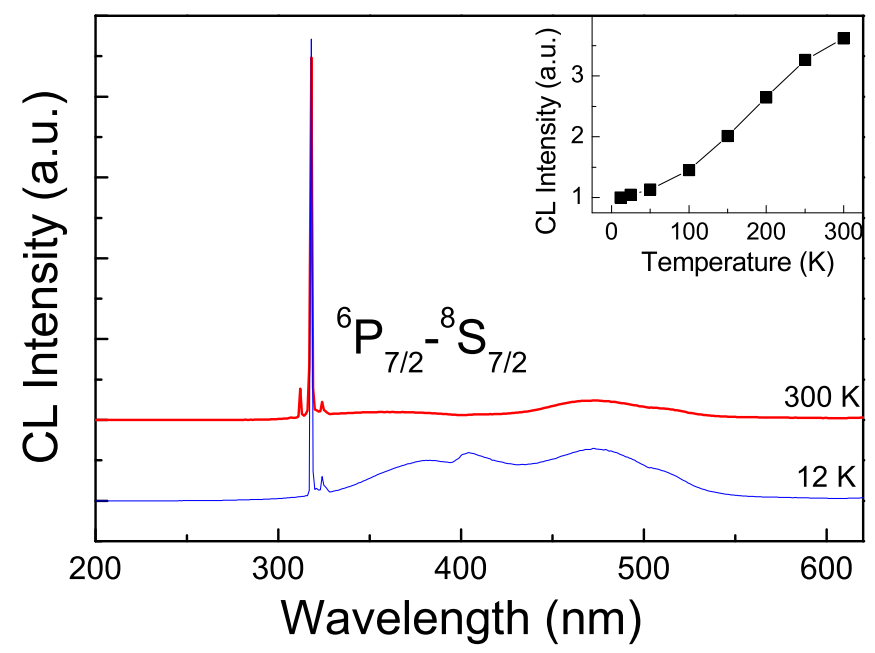

Figure 5.2: Cathodoluminescence spectrum of Gd implanted AlN [44], measured at $12 \mathrm{~K}$ (lower spectrum) and $300 \mathrm{~K}$ (upper spectrum) under 5 $\mathrm{keV}, 0.3 \mathrm{~W} / \mathrm{cm}^{2}$ electron excitation. The spectrum was recorded with a photomultiplier and corrected for the response function of the setup. Inset: temperature-dependent integral intensity of the ${ }^{6} P_{7 / 2} \rightarrow{ }^{8} S_{7 / 2}$ transition.

spectrum at around $600 \mathrm{~nm}$ [162]. The fact that these transition from ${ }^{6} G_{7 / 2}$ are not detectable is a first indication that this level and all the levels above, whose energies fall into the band gap of the AlN host, are not excited.

The inset in Figure 5.2 shows the evolution of the integral intensity of the ${ }^{6} \mathrm{P}_{7 / 2} \rightarrow{ }^{8} \mathrm{~S}_{7 / 2}$ transition with increasing temperature. Between $12 \mathrm{~K}$ and $300 \mathrm{~K}$ an increase by a factor of 3.5 is detected. This behaviour will be discussed later in combination with the time-resolved measurements.

Figure 5.3 shows high resolution cathodoluminescence spectra, focusing on the strong luminescence discussed above, measured at $12 \mathrm{~K}, 77 \mathrm{~K}$ and $300 \mathrm{~K}$. The strong line is resolved into four individual components, all belonging to the ${ }^{6} P_{7 / 2}$ multiplet. This is based on the observation that with increasing temperature an increase in the intensities of the energetically higher lying levels within the multiplet is detected. This behavior meets exactly the expectations, according to the fractional thermal population of doubly degenerate levels. The splitting of the ground state ${ }^{8} S_{7 / 2}$ stays unresolved. The ${ }^{6} P_{7 / 2} \rightarrow{ }^{8} S_{7 / 2}$ transition is accompanied by side bands, at low temperatures restricted to the lower energies; side bands of higher energies are only detected in the $300 \mathrm{~K}$ spectrum. Looking closely at the structure of these side bands, it can be seen that some of them reflect the splitting as well as the fractional thermal population of the ${ }^{6} P_{7 / 2}$ level, and one may draw the conclusion that these side bands are photonic in nature rather than arising from $\mathrm{Gd}^{3+}$ ions on other sites. The side band highest in energy does not resemble the ${ }^{6} P_{7 / 2}$ level. Instead, a splitting into three lines suggests that this is the ${ }^{6} P_{5 / 2}$ multiplet, which is thermally populated at higher temperatures. 


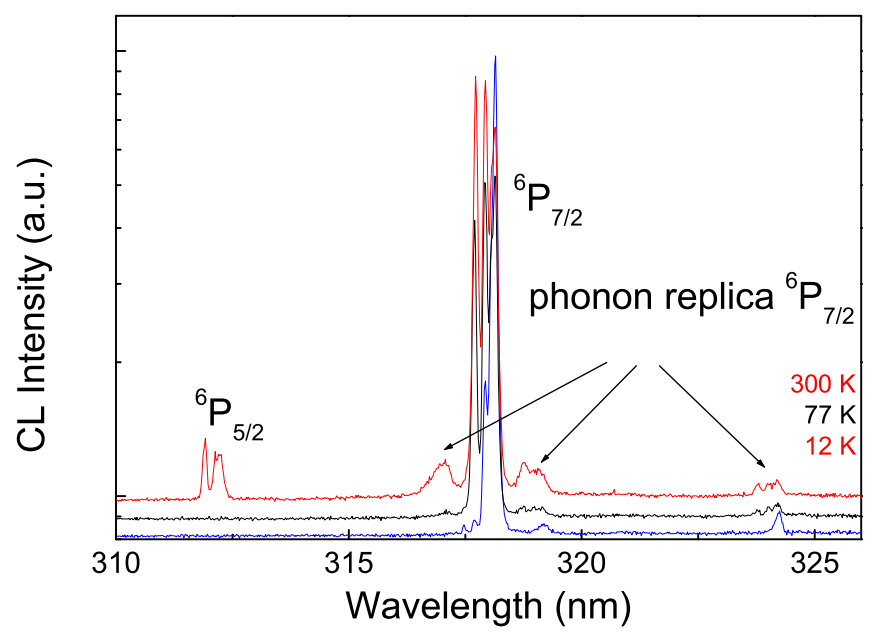

Figure 5.3: The ${ }^{6} P_{7 / 2} \rightarrow{ }^{8} S_{7 / 2}$ transition and phonon sidebands at $12 \mathrm{~K}$, $77 \mathrm{~K}$ and $300 \mathrm{~K}$ [44].

Time-resolved cathodoluminescence spectroscopy was performed monitoring the strongest transition at $318.4 \mathrm{~nm}$. In Figure 5.4 the decay curves measured at $300 \mathrm{~K}$ and $12 \mathrm{~K}$ are shown. The decay can be described by two independent decays, one of them showing energy transfer. Because energy levels above the ${ }^{6} \mathrm{P}_{7 / 2}$ are weak, the rate equations describing the decay of the ${ }^{6} \mathrm{P}_{7 / 2}$ level correspond to a two-level system. Inokuti et al [140] derived an expression for the population of such an excited level in the case of energy transfer (compare equation 3.51. Taking into account a second independent decay, the following modified equation for the description of the depopulation of the ${ }^{6} \mathrm{P}_{7 / 2}$ level can be used:

$$
I(t)-I_{B G}=I_{1} e^{\left(-t / \tau_{1}+A\left(-t / \tau_{1}\right)^{3 / s}\right)}+I_{2} e^{-t / \tau_{2}}
$$

where $I_{B G}$ represents the background intensity, $I_{1}$ and $N_{2}$ refer to the initial equilibrium intensities of both sites, $\tau_{1}$ and $\tau_{2}$ are the lifetimes, $s$ is the coupling constant which describes the type of energy transfer, and $A$ is a parameter depending on $s$ and the concentration of the ions. Because equation 5.1 only weakly depends on $s$, this parameter was fixed to 6, corresponding to a dipole-dipole interaction. The parameter $A$ was also constrained during the fitting procedure as well. The fit obtained in this way is shown in Figure 5.4. On the right-hand side of figure 5.4 the extracted lifetimes are shown. The longer lifetime corresponds to the decay $I_{2} e^{-t / \tau_{2}}$ of equation 5.1. but $I_{2}$ accounts for only $2 \%$ of the total intensity. In principle this second lifetime has to be attributed to a second site of the ions.

The main fraction (98\%) of the ions can be attributed to the first part of equation 5.1. As can be seen from figure 5.4, the lifetime of the ${ }^{6} \mathrm{P}_{7 / 2}$ level decreases from $0.76 \mathrm{~ms}$ at $12 \mathrm{~K}$ to $0.69 \mathrm{~ms}$ at $300 \mathrm{~K}$. A temperature-dependent lifetime $\tau(T)$ is generally expected for a multiplet split 

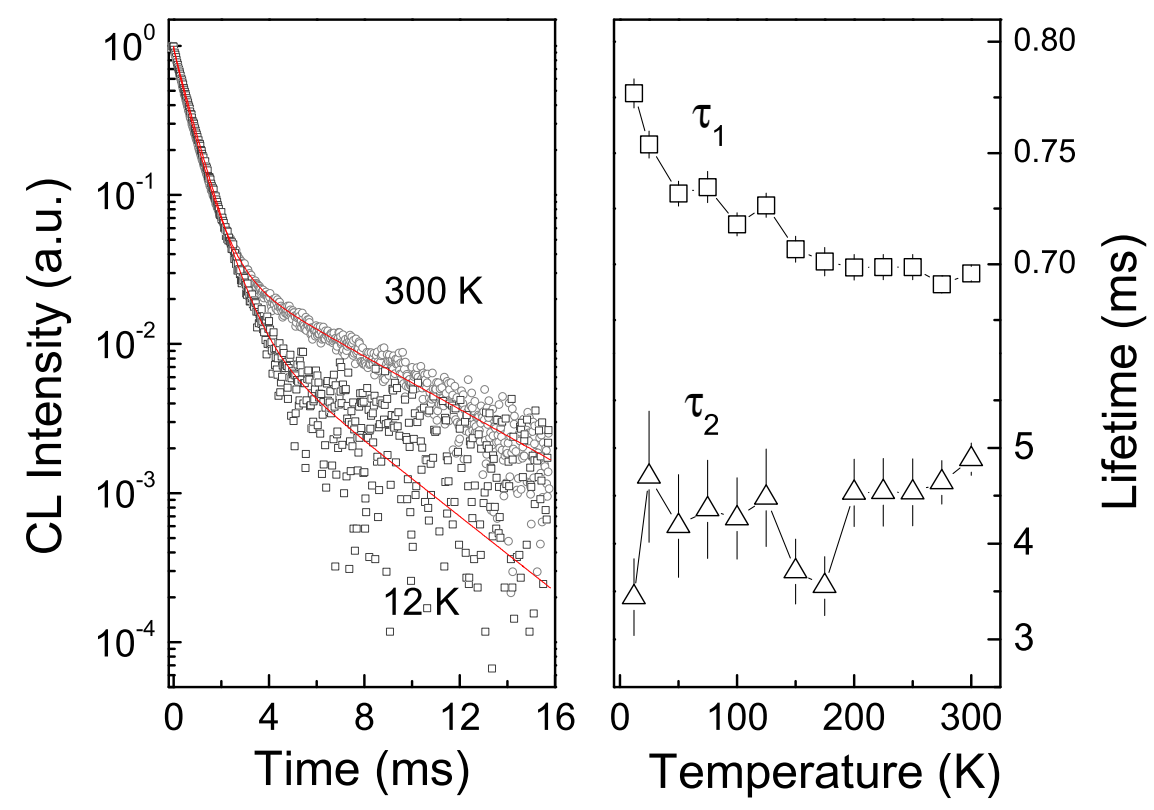

Figure 5.4: Luminescence decay of the ${ }^{6} P_{7 / 2}$ level of $\mathrm{Gd}^{3+}$ in $\mathrm{AlN}$ [44].

by the crystal field because of the fractional thermal population $X(i)$ of the initial Stark levels (compare equation 3.44). Another possible decrease in lifetime with increasing temperature is expected because of the emission of phonons.

The decrease in lifetime with increasing temperature can directly be compared to the increasing integral intensity of the ${ }^{6} \mathrm{P}_{7 / 2} \rightarrow{ }^{6} \mathrm{P}_{7 / 2}$ transition and it is obvious that one cannot account for the increase in intensity by the decrease in lifetime. The intensity of the ${ }^{6} P_{7 / 2} \rightarrow{ }^{8} S_{7 / 2}$ can be modeled in the scope of a two-level system, described by the well-known formula

$$
I\left({ }^{6} P_{7 / 2}\right) \sim N\left({ }^{6} P_{7 / 2}\right)=\frac{\sigma \tau I N}{1+\sigma \tau I}
$$

where $\sigma$ refers to the cross section of the excitation, $\tau$ to the lifetime of the ${ }^{6} P_{7 / 2}$ level, $I$ to the flux of the exciting particles and $\mathrm{N}$ to the overall number of $\mathrm{Gd}^{3+}$ ions in a specific volume. This assumption is reasonable as the intensity of the ${ }^{6} P_{7 / 2} \rightarrow{ }^{8} S_{7 / 2}$ transition indeed depends on the excitation power as predicted by equation 5.2 [44]. On the contrary, one may conceive that the increase in intensity with increasing temperature can be attributed to the excitation mechanism. 


\subsection{3 $\mathbf{E u}^{3+}$ doped AIN}

In order to investigate the cathodoluminescence of Eu-implanted $\mathrm{AlN},{ }^{151} \mathrm{Eu}^{+}$ions were implanted at the implantation facility IONAS at energies of $100 \mathrm{keV}$ and fluences of 1 . $10^{13}$ ions $/ \mathrm{cm}^{2}$. The luminescence behavior of europium implanted AlN has been studied earlier by Jadwisienczak et al. [43], although the annealing conditions were different from the ones used in this work. Jadwisienczak et al. performed annealing of the samples under $\mathrm{N}_{2}$ flow in a tube furnace at temperatures up to $1373 \mathrm{~K}$. The vacuum annealing conditions chosen for the AlN substrates in this work are based on the experience that annealing under $\mathrm{N}_{2}$ flow leads to incorporation of impurities such as $\mathrm{Cr}^{3+}$, or even partial destruction of the AlN film.

Subsequent to implantation, the samples were annealed under vacuum conditions $\left(\sim 10^{-6}\right.$ mbar $)$ at $1373 \mathrm{~K}$ for 30 minutes in a tube furnace. The $\mathrm{CL}$ survey scans, recorded at $12 \mathrm{~K}$ as well as $300 \mathrm{~K}$ are shown in figure 5.5 .

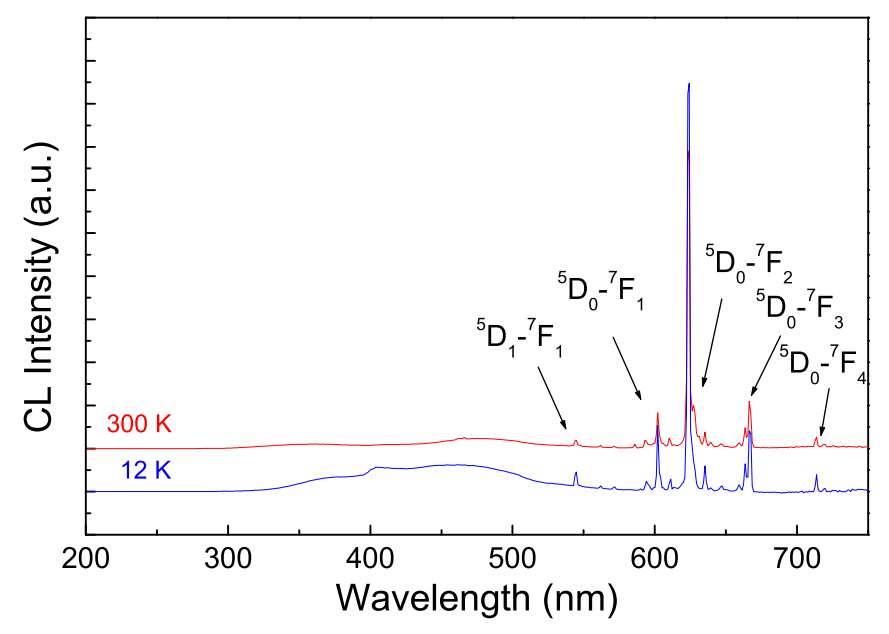

Figure 5.5: Cathodoluminescence survey scans of $\mathrm{Eu}^{3+}$ in $\mathrm{AlN}$, measured at $12 \mathrm{~K}$ and $300 \mathrm{~K}$. The most important transitions are assigned.

Besides the defect-related luminesence of the AlN host at around $450 \mathrm{~nm}$, sharp luminescence lines corresponding to a radiative intra-4f electron transition of $\mathrm{Eu}^{3+}$ are visible. Based on the energy level diagram of $\mathrm{Eu}^{3+}$ (see Figure 3.2), these luminescence lines are assigned to the ${ }^{5} D_{1} \rightarrow{ }^{7} F_{1}$ as well as ${ }^{5} D_{0} \rightarrow{ }^{7} F_{J, J=1,2,3,4}$ intra-4f electron transitions of $\mathrm{Eu}^{3+}$. The strongest lines at around $623 \mathrm{~nm}$ belong to the ${ }^{5} D_{0} \rightarrow{ }^{7} F_{2}$ transition. This could be calculated with the help of the mean free ion parameters for $\mathrm{Eu}^{3+}$ listed in table A.1. It is a well-known fact that the branching ratios only significantly change in case of inversion symmetry, where only magnetic dipole transitions, such as ${ }^{5} D_{0} \rightarrow{ }^{7} F_{1}$, are allowed. From the energy level diagram of $\mathrm{Eu}^{3+}$, shown in figure 3.2 , it can be seen that many energy levels are situated within the band gap of 


\subsection{ALUMINUM NITRIDE}

the AlN host, but most of them are spaced so closely that non-radiative depopulation to levels energetically located below is dominant.

The limited number of detectable transitions in CL spectroscopy makes it impossible to perform a crystal field analysis, even though $\mathrm{Eu}^{3+}$ is an even-numbered $4 f$ electron system. In Figure 5.6 high resolution Stark level spectra are shown for the ${ }^{5} D_{0} \rightarrow{ }^{7} F_{1},{ }^{5} D_{1} \rightarrow{ }^{7} F_{1},{ }^{5} D_{0} \rightarrow{ }^{7} F_{0}$, as well as ${ }^{5} D_{1} \rightarrow{ }^{7} F_{0}$ transitions. It is a common approach to identify transitions on the basis of their final multiplets of the transitions (such as ${ }^{7} F_{1}$ and ${ }^{7} F_{0}$ in this case). They appear repeatedly, but still with differences in intensity and some lines may be missing in case group theoretical selection rules forbid transitions under certain symmetries. In can be seen from figure 5.6 that

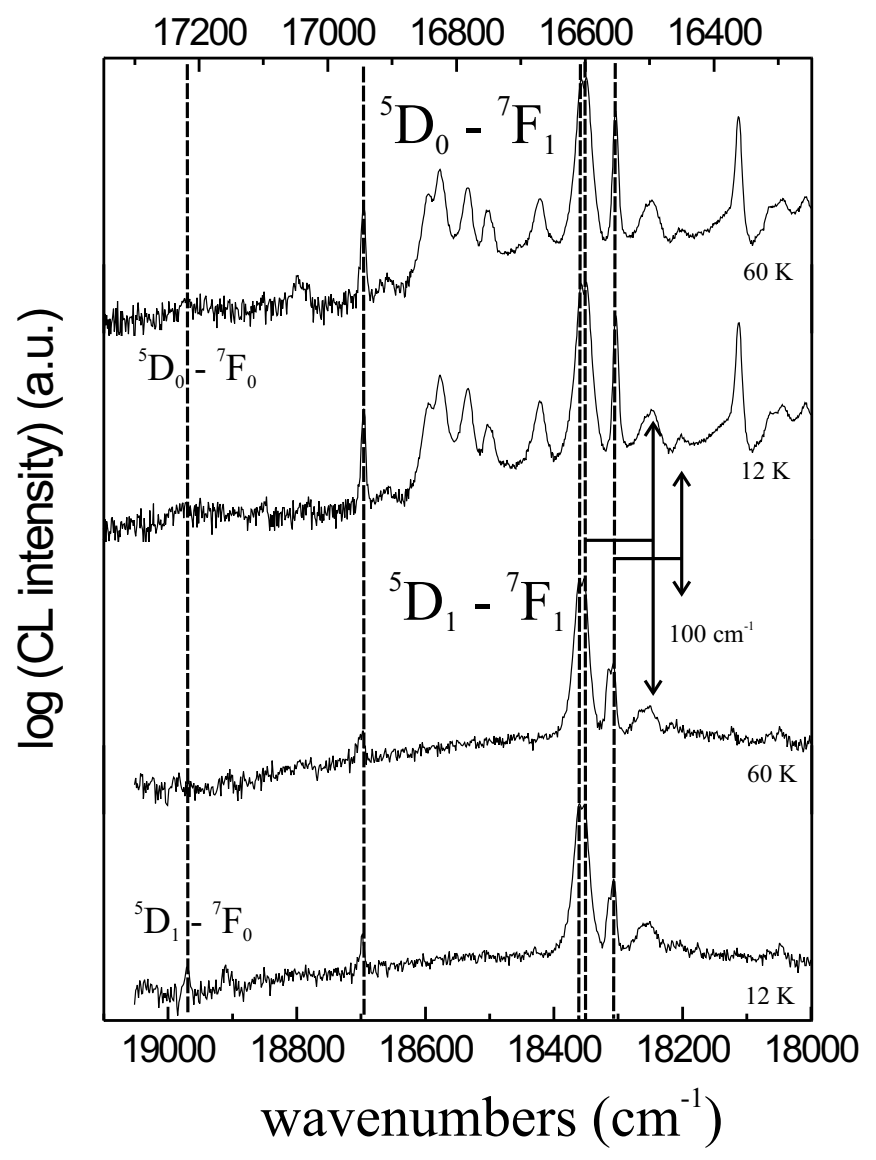

Figure 5.6: High resolution spectra of the ${ }^{5} D_{0} \rightarrow{ }^{7} F_{1}$ and ${ }^{5} D_{1} \rightarrow{ }^{7} F_{1}$ transitions of $\mathrm{Eu}^{3+}$ in $\mathrm{AlN}$, measured at $12 \mathrm{~K}$ and $60 \mathrm{~K}$. The spectra and lines are shown to visualize the search for repeating patterns in the luminescence spectra.

even for transitions between multiplets with small $J$-values the spectra can take complicated forms, and the assignment is not as straightforward as one might expect. The ${ }^{5} D_{0} \rightarrow{ }^{7} F_{1}$ transition is accompanied by numerous weak lines which probably belong to transitions from levels above the ${ }^{5} D_{0}$ to the ${ }^{7} F_{J}$ multiplets. Even the local mode phonon side bands, which could be resolved well in the case of $\mathrm{Gd}^{3+}$-doped AlN (see chapter 5.1.2) are relatively broad. The 
energy of these side bands is determined with a relatively large inaccuracy to $100 \mathrm{~cm}^{-1}$.

Finally, lifetime measurements of the ${ }^{5} D_{0}$ were performed monitoring the ${ }^{5} D_{0} \rightarrow{ }^{7} F_{2}$ transition. The corresponding decay curves at $12 \mathrm{~K}$ as well as $300 \mathrm{~K}$ are shown in Figure 5.7. The decay curves are complex and are neither described by a single exponential function (see equation 3.49 , nor by assuming population from higher lying levels such as in the case of the ${ }^{1} D_{2}$ level for $\mathrm{Tm}^{3+}$ in AlN (see chapter 5.1.4), nor by assuming energy transfer between $\mathrm{Eu}^{3+}$ ions (see equation 3.51). Because the higher lying level ${ }^{5} D_{1}$ does not dominantly decay non-radiatively,

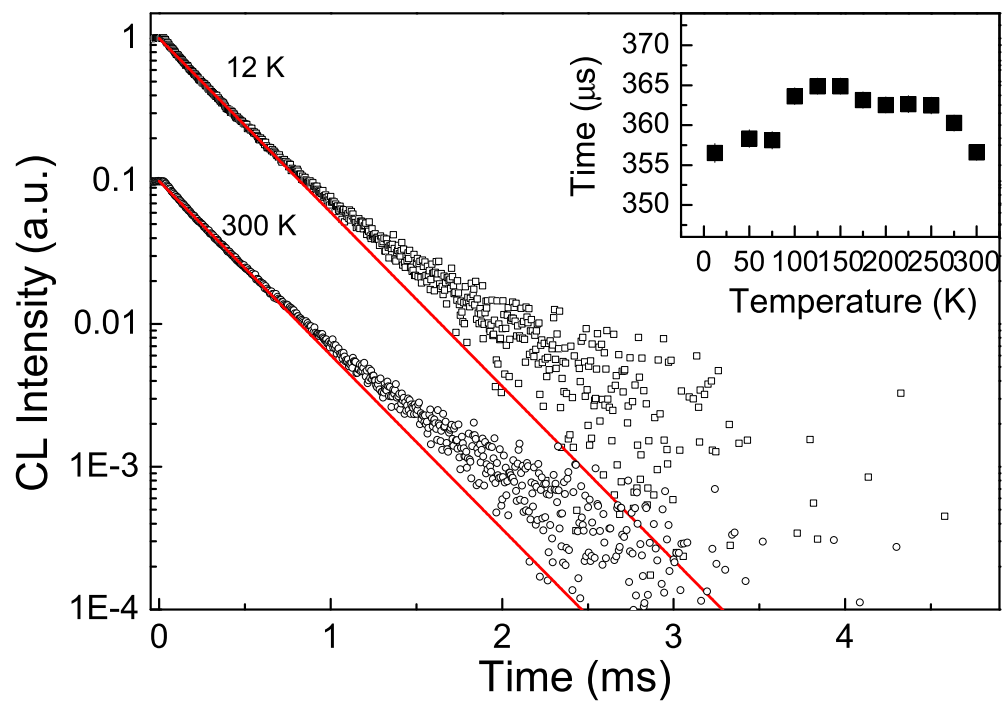

Figure 5.7: Luminescence decay of the ${ }^{5} D_{0}$ level of $\mathrm{Eu}^{3+}$ in $\mathrm{AlN}$ in the temperature range $12 \mathrm{~K}-300 \mathrm{~K}$. The decay curves, shifted in intensity in the case of the $300 \mathrm{~K}$ spectrum, are fitted to a single exponential function which reasonably describes the initial curves. For later times a derivation is visible, the origin of which is discussed in the text.

it is reasonable to assume that energy transfer as well as population from higher lying levels alter the decay curve of the ${ }^{5} D_{0}$ level. Because an evaluation would require too many fitting parameters, the decay curve is fitted to a single exponential decay. The inset of Figure 5.7 shows the extracted lifetime of the ${ }^{5} D_{0}$ level which is in the range of about $360 \mu$ s. This value is somewhat lower than the one reported by Jadwisienczak et al. (408.5 $\mu$ s at $300 \mathrm{~K})$, the difference can be attributed to different fitting approaches.

\subsection{4 $\mathbf{T m}^{3+}$ in AlN - blue light emission at room temperature}

Some of the luminescence investigations reported in the following were performed with the samples used for emission channeling studies, as described in chapter 5.1.1. Additionally, stable ${ }^{169} \mathrm{Tm}$ ions were implanted at the IONAS facility at an energy of $100 \mathrm{keV}$ and fluences of $1 \cdot 10^{13}$ ions $/ \mathrm{cm}^{2}$. Subsequent to implantation the samples were annealed for 30 minutes un- 


\subsection{ALUMINUM NITRIDE}

der vacuum conditions $\left(\sim 10^{-6}\right.$ mbar $)$ at $1373 \mathrm{~K}$. These samples are used for the crystal field analysis reported in chapter 7 .

Figure 5.8 shows the survey scan recorded at $12 \mathrm{~K}$ as well as $300 \mathrm{~K}$. It can be seen that at $12 \mathrm{~K}$ the spectrum is dominated by several transitions mainly between $290 \mathrm{~nm}$ and $490 \mathrm{~nm}$ as well as $805 \mathrm{~nm}$ with weaker transitions in between. Most of the luminescence is assigned to radiative intra-4f electron transitions from the ${ }^{1} I_{6},{ }^{1} D_{2}$, and ${ }^{1} G_{4}$ levels to levels below (compare the energy level diagram shown in Figure 3.2). The assignment of these transitions is complicated in many cases and is mainly a result of lifetime measurements as well as the crystal field calculations which will be reported in chapter 7

At $300 \mathrm{~K}$ the spectrum is mainly dominated by the strong ${ }^{1} D_{2} \rightarrow{ }^{3} F_{4}$ transition at around $465 \mathrm{~nm}$ and several strong transitions around $800 \mathrm{~nm}$. It is obvious that the spectrum is very different from the one at $12 \mathrm{~K}$. Such an extreme change is indeed very rare. To understand this behavior, time-resolved measurements were performed on the strongest transitions found in the spectra, namely ${ }^{1} I_{6} \rightarrow{ }^{3} H_{6}$ and ${ }^{1} D_{2} \rightarrow{ }^{3} F_{4}$. The corresponding lifetime measurements are summarized in Figure 5.9. It was discovered that the decay of the ${ }^{1} I_{6}$ level is well described by a single

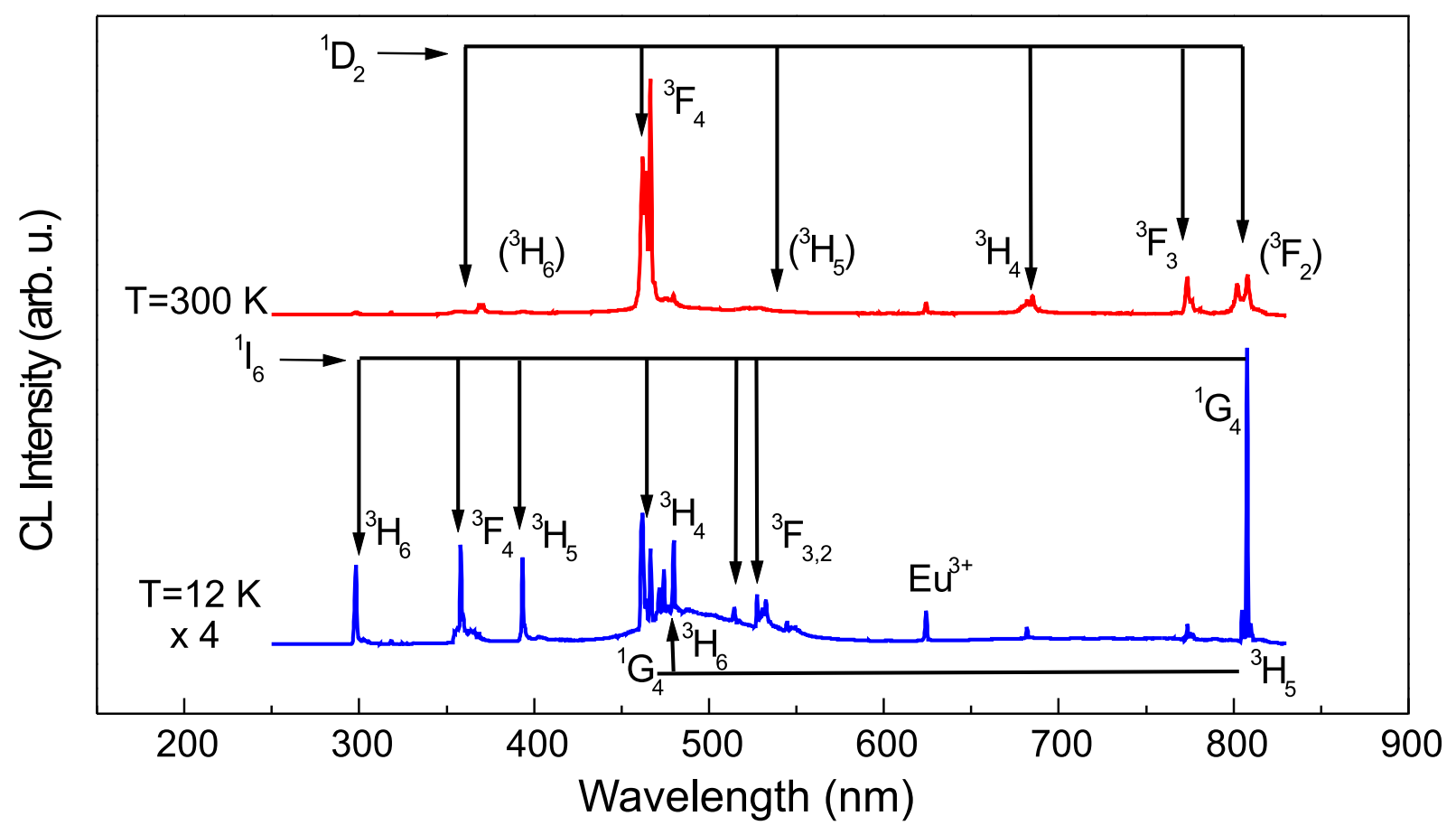

Figure 5.8: Cathodoluminescence spectra of Tm implanted AIN, measured at $12 \mathrm{~K}$ (lower spectrum) and $300 \mathrm{~K}$ (upper spectrum) under $5 \mathrm{keV}, 0.3 \mathrm{~W} / \mathrm{cm}^{2}$ electron excitation. The spectrum was recorded with a photomultiplier and corrected for the response function of the setup. 
(a)

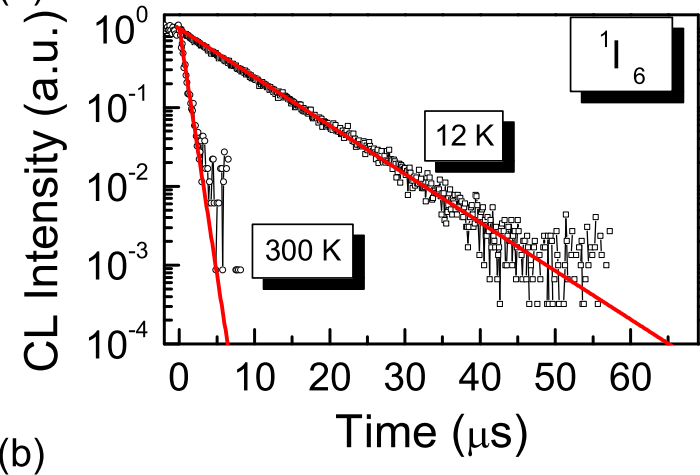

(b)

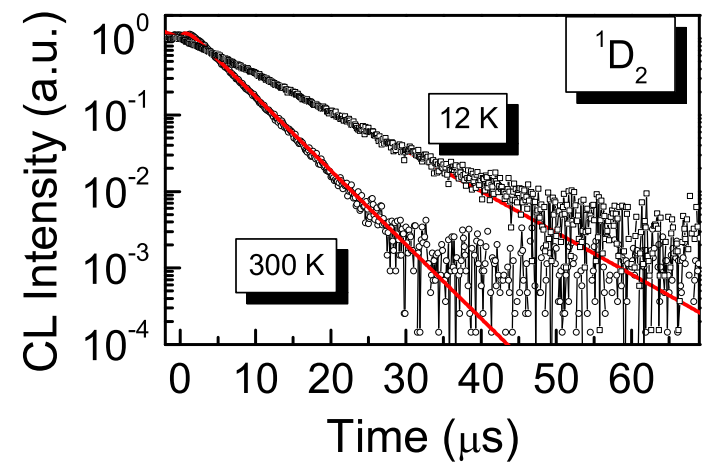

(c)
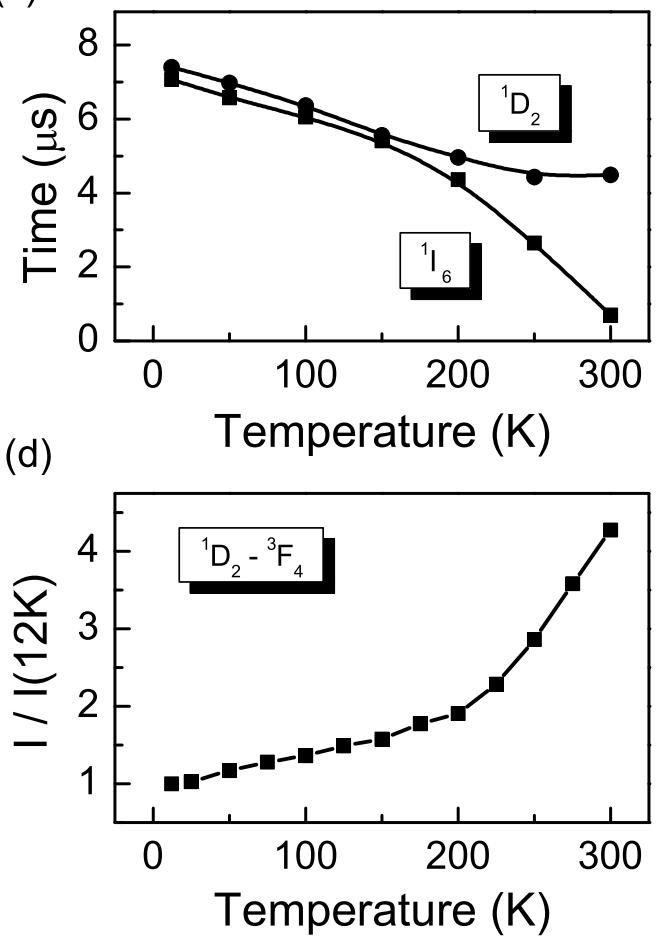

Figure 5.9: Luminescence decay of the ${ }^{1} I_{6}$ and ${ }^{1} D_{2}$ levels of $\mathrm{Tm}^{3+}$ in AlN, measured in the temperature range $12 \mathrm{~K}$ to $300 \mathrm{~K}$. Figures (a) and (b) show the corresponding decay curves at $12 \mathrm{~K}$ and $300 \mathrm{~K}$, the solid lines represent the fit as described in the text. Figure (c) shows the temperature dependence of the lifetimes of the ${ }^{1} I_{6}$ and ${ }^{1} D_{2}$, figure (d) the integral intensity of the ${ }^{1} D_{2} \rightarrow{ }^{3} F_{4}$ transition.

exponential decay, while the decay of the ${ }^{1} D_{2}$ level shows both depopulation and population at the same time. Because the ${ }^{1} D_{2}$ level is energetically located below the ${ }^{1} I_{6}$ level, it is reasonable to assume that the latter decays to a large extent to the ${ }^{1} D_{2}$ level, giving rise to an observable population. Both levels can be modeled by the following set of rate equations which are a specific form of equation 3.46 .

$$
\frac{d}{d t}\left(\begin{array}{c}
N^{{ }_{1}} I_{6} \\
N^{1} D_{2}
\end{array}\right)=\left(\begin{array}{cc}
-\left(\tau_{1} I_{6}\right)^{-1} & 0 \\
c\left(\tau_{1} I_{6}\right)^{-1} & -\left(\tau_{1} D_{2}\right)^{-1}
\end{array}\right)\left(\begin{array}{c}
N_{{ }^{1} I_{6}} \\
N^{{ }_{1}} D_{2}
\end{array}\right)
$$

The fitting constant $c$ is included to account for the fact that the ${ }^{1} I_{6}$ level only partially decays to the ${ }^{1} D_{2}$ level. The temperature dependent lifetime of the ${ }^{1} I_{6}$ level is directly extracted from the experimental data (see Figure 5.9 (a)), while the lifetime of the ${ }^{1} D_{2}$ level is obtained by 


\subsection{ALUMINUM NITRIDE}

fitting the experimental decay curve to the integral solution of equation 5.3 (see Figure 5.9(b)). This includes the lifetime of the ${ }^{1} I_{6}$ level which was kept fixed during the fitting procedure according to the corresponding experimental lifetime. The temperature dependent lifetimes of both the ${ }^{1} I_{6}$ and the ${ }^{1} D_{2}$ level are shown in Figure 5.9 (c). While both lifetimes are decreasing with increasing temperature, the lifetime of the ${ }^{1} I_{6}$ level drops below $1 \mu \mathrm{s}$, thus due to a strong non-radiative decay. The lifetime of the ${ }^{1} D_{2}$ level is almost constant above $275 \mathrm{~K}$. One should keep in mind that fractional thermal population of initial Stark levels may alter the lifetime (see equation 3.45) and it is therefore almost impossible to numeralize the fraction of the nonradiative decay of the ${ }^{1} I_{6}$ level. The fact, that the drop in lifetime of the ${ }^{1} D_{2}$ level remains static at higher temperatures, is a strong indication of a decrease in lifetime due to fractional thermal population. Indeed, the crystal field splitting of the ${ }^{1} D_{2}$ level often shows two closely spaced Stark levels lowest in energy and far separated from higher lying levels of the ${ }^{1} D_{2}$ multiplet (compare e.g. Ref. [100]). The lifetime of the ${ }^{1} D_{2}$ level was measured or calculated for many host matrices, the reported values range from $9.1 \mu$ s for $\mathrm{Tm}^{3+}$ in $\mathrm{Sr}_{5}\left(\mathrm{PO}_{4}\right)_{3} \mathrm{~F}$ [250] up to $121.8 \mu \mathrm{s}$ for $\mathrm{Tm}^{3+}$ in $\mathrm{Tm}\left(\mathrm{NO}_{3}\right)_{3} \cdot 5 \mathrm{H}_{2} \mathrm{O}$ [161]. It is not possible to estimate the quantum efficiency for the radiative decay of the ${ }^{1} D_{2}$ level of $\mathrm{Tm}^{3+}$ in AlN, because the $\Omega_{\lambda}$ intensity parameters (see equation 3.32, which have a strong influence on the lifetime of the ${ }^{1} D_{2}$ level, may vary extremely from host to host [152, 251].

At present it is unclear why the ${ }^{1} I_{6}$ level decays non-radiatively to the ${ }^{1} D_{2}$ level. However, the radiative transition probability for the ${ }^{1} I_{6} \rightarrow{ }^{1} D_{2}$ transition is too small (compare e.g. Ref. [161]) to significantly change the decay curve of the ${ }^{1} D_{2}$ level. With regards to multi-phonon relaxation one should keep in mind that the energetic distance between the ${ }^{1} D_{2}$ and the ${ }^{1} I_{6}$ level is larger than $6,000 \mathrm{~cm}^{-1}$, which can only be bridged by more than seven phonons in the AlN host (compare figure 3.5), a process which is rather unlikely. Jadwisienczak et al. [43] observed a similar behavior of $\mathrm{Tb}^{3+}$ in $\mathrm{AlN}$, where an energetic distance of more than 5,000 $\mathrm{cm}^{-1}$ between the ${ }^{5} D_{3}$ and ${ }^{5} D_{4}$ levels of $\mathrm{Tb}^{3+}$ is bridged. The possible explanations given by Jadwisienczak et al. are the presence of $\mathrm{OH}$-groups with vibrational frequencies of 3,750 $\mathrm{cm}^{-1}$ or cross-relaxation processes. However, there is still another possible explanation of the observed down-conversion, which is the involvement of excitons. In principle, the decay ${ }^{1} I_{6} \rightarrow{ }^{1} D_{2}$ could involve backtransfer from the ${ }^{1} I_{6}$ level and again transfer to the ${ }^{1} D_{2}$ level. It is unclear whether the properties of the AlN film, such as its thickness of only $200 \mathrm{~nm}$, are involved in the down-conversion process or not. 

CARBIDES

\subsubsection{Summary}

Conversion electron emission channeling studies of ${ }^{169} \mathrm{Yb}$ ions revealed a substitutional $\mathrm{Al}$ (cationic) site for the implanted ions. Due to the fact that in this case the implanted ions replace a trivalent $\mathrm{Al}$ atom, the same lattice site is suggested for other lanthanide ions such as thulium or gadolinium.

Gadolinium implanted AlN, annealed at $1373 \mathrm{~K}$ under vacuum conditions, revealed to be a very promising candidate for the implementation of ultra violet light emitters. The dominant luminescence related to $\mathrm{Gd}^{3+}$ was monitored at $318 \mathrm{~nm}$. It can be attributed to the ${ }^{6} P_{7 / 2} \rightarrow{ }^{8} S_{7 / 2}$ intra- $4 f$ electron transition of $\mathrm{Gd}^{3+}$ between the first excited state and the ground state. Lifetime measurements of the ${ }^{6} P_{7 / 2}$ level show a slightly decreasing lifetime with increasing temperature, reaching $0.69 \mathrm{~ms}$ at $300 \mathrm{~K}$. This decrease in lifetime was compared to the increase in the integral intensity of the ${ }^{6} P_{7 / 2} \rightarrow{ }^{8} S_{7 / 2}$ transition with increasing temperature. It was shown that the increasing intensity must be attributed to the excitation mechanism.

Europium-implanted AlN, annealed at $1373 \mathrm{~K}$ under vacuum conditions, shows characteristic luminescence due to intra $-4 f$ electron transitions of $\mathrm{Eu}^{3+}$ at around $620 \mathrm{~nm}$, making this system an interesting candidate for red light emitters. Is was exemplarily shown for the case of the ${ }^{5} D_{0} \rightarrow{ }^{7} F_{1}$ and ${ }^{5} D_{1} \rightarrow{ }^{7} F_{1}$ transitions, how final multiplets of transitions in luminescence spectra are identified. Lifetime measurements monitoring the dominant ${ }^{5} D_{0} \rightarrow{ }^{7} F_{2}$ transition show an almost constant lifetime around $360 \mu$ s over the temperature range $12 \mathrm{~K}$ to $300 \mathrm{~K}$.

Thulium implanted AlN, annealed at $1273 \mathrm{~K}$ under vacuum conditions, showed a dominant blue light emission at around $465 \mathrm{~nm}$. Based on lifetime measurements and the crystal field analysis described later in this work, the dominating blue emission at $300 \mathrm{~K}$ is attributed to the ${ }^{1} D_{2} \rightarrow{ }^{3} F_{4}$ intra- $-4 f$ electron transition of $\mathrm{Tm}^{3+}$. The survey spectra, recorded at $12 \mathrm{~K}$ and $300 \mathrm{~K}$, revealed remarkable differences. While at $12 \mathrm{~K}$, the $\mathrm{Tm}^{3+}$-related luminescence is dominated by transitions starting from the ${ }^{1} I_{6}$ level, the luminescence at $300 \mathrm{~K}$ is dominated by transitions starting from the ${ }^{1} D_{2}$ level. Lifetime measurements of both initial levels suggest that with increasing temperature the ${ }^{1} I_{6}$ level is depopulated non-radiatively to the ${ }^{1} D_{2}$ level, where it efficiently populates the latter. 


\subsection{Cubic boron nitride}

The synthesis of boron nitride's most promising candidate, the cubic phase c-BN, has made tremendous progress during the last decade. Nowadays it is possible to synthesize large bulk specimen of high quality [252, 253] under high temperature/high pressure (HTHP) conditions, as well as high-quality c-BN thin films [254]. Showing high thermal conductivity and stability, extreme chemical inertness, and a band gap of $6.1-6.4 \mathrm{eV}$ [134], the III-V-semiconductor $\mathrm{c}-\mathrm{BN}$ is very attractive for the implementation of light emitters. The first realization of c-BN based light emitting pn-junctions [255, 256] shows that research in this direction has stricken promising new paths.

The conditions under which c-BN can be synthesized are as extreme as its physical properties. The growth of layers of $\mathrm{c}-\mathrm{BN}$ on substrates is bound to the use of energetic ions and the thin films obtained in such a way are nano crystalline. Among the growth methods applied, massselected ion beam deposition (MSIBD) is a very attractive approach for the synthesis of pure c-BN films under UHV conditions. An overview of current research on MSIBD-grown c-BN was given by Feldermann [257].

In recent years, tremendous progress has been made to synthesize single crystal [253] and pure poly crystalline c-BN [252] under high pressure and high temperature conditions. In the case of poly crystalline c-BN, large samples consisting of small crystals of a few $\mathrm{mm}$ in size and of high purity can be synthesized. Single crystals are usually grown using solvents, resulting in small crystal with maximum diameters of a few $\mathrm{mm}$. Usually, such crystals contain many different impurities. A photograph of the single crystals used in this work is shown in figure 5.10. These single crystals, together with the poly crystalline specimen, were kindly supplied by T. Taniguchi (National Institute for Research in Inorganic Materials (NIRIM), Japan).

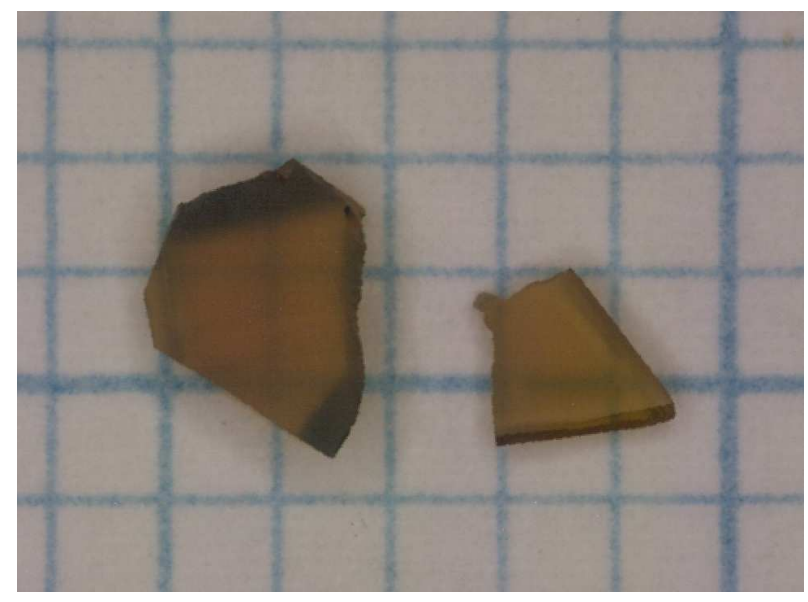

Figure 5.10: Photograph of c-BN single crystals used for EC experiments. The crystals were grown at $1773 \mathrm{~K}, 5.5$ GPa using $\mathrm{Li}_{3} \mathrm{BN}_{2}$ and $\mathrm{Ca}_{3} \mathrm{~B}_{2} \mathrm{~N}_{4}$ as solvents. The size of the squares is $1 \times 1$ $\mathrm{mm}^{2}$. 
Nothing is known about the annealing behavior of implantation-related defects in cubic boron nitride after heavy ion implantation. The usual approach in such a case is to apply PAC using

${ }^{111}$ In as probe atoms, which was also done in some experiments. This did not yield any results for annealing temperatures up to $1473 \mathrm{~K}$, which points to a strong threshold as regards the temperature necessary to recover implantation defects in the $\mathrm{c}-\mathrm{BN}$ host material. Several questions stay unanswered because of this, such as choice of the annealing temperature and pressure after ion implantation, which is rather based on intuition than on facts.

\subsubsection{Lattice location of ${ }^{139} \mathrm{Ce}$ in c-BN}

For the lattice location studies of ${ }^{139} \mathrm{Ce}$ applying the emission channeling (EC) technique, small single crystals were used. These crystals are shown in figure 5.10. Radioactive ${ }^{139} \mathrm{Ce}$ ions at an energy of approx. $60 \mathrm{keV}$ and a fluence of $1 \cdot 10^{13} \mathrm{ions} / \mathrm{cm}^{2}$ were implanted at the online isotope separator ISOLDE. The decay chain is described in section 3.2.5. A beam collimator with a diameter of $1 \mathrm{~mm}$ was used for implantation to define the angular resolution of the measurement. After implantation the samples were annealed at $1173 \mathrm{~K}$ in an evacuated quartz ampoule for 20 minutes. Emission channeling spectra were recorded using the conversion electrons arising in the electron capture decay ${ }^{139} \mathrm{Ce} \rightarrow{ }^{139} \mathrm{La}\left(\mathrm{t}_{1 / 2}=137.63 \mathrm{~d}\right)$. The conversion electron energy spectrum and the decay scheme are shown in figure 3.2.5. The EC measurements were performed using a 2-dimensional electron detector, as described in Refs. [258, 213]. Figure 5.11 shows the conversion electron EC patterns of ${ }^{139} \mathrm{Ce}$ in c-BN arising in the decay of ${ }^{139} \mathrm{Ce} \rightarrow{ }^{139} \mathrm{La}$. EC spectra were recorded for the $\langle 100\rangle$ as well as the $<211\rangle$ axis. It is evident that the maximum channeling yield is quite low, indicating that only a small fraction of emitters is located on substitutional sites. Especially the spectrum around the $<211>$ axis, where the usually pronounced planar channeling effect, belonging to the $\{111\}$ plane, is completely absent, indicates that the lattice location of the emitters cannot be reproduced by one single, highly symmetric site, neither substitutional nor interstitial. As for both $<100>$ and $<211>$ directions an axial channeling effect is visible, numerous calculations were performed assuming that the $\mathrm{Ce}$ ions are displaced from an ideal substitutional boron site towards other sites of higher symmetry, such as the so called bond-center, anti-bond-center, and split sites. None of these calculations resulted in a reasonable agreement with the experimental spectra. One possibility to account for the absent planar channeling effect of the $\{111\}$ plane is to assume that emitter atoms are incorporated in both substitutional boron and tetrahedral interstitial sites at a ratio of $2: 1$, surrounded in both cases by four nitrogen ligands. Only a fraction of $15 \%$ of the implanted ions are then located on these sites; the remainder is assumed to stay on sites of very low symmetry, so-called "random" sites. The resulting simulations are shown on the right-hand side of figure 5.11. The 

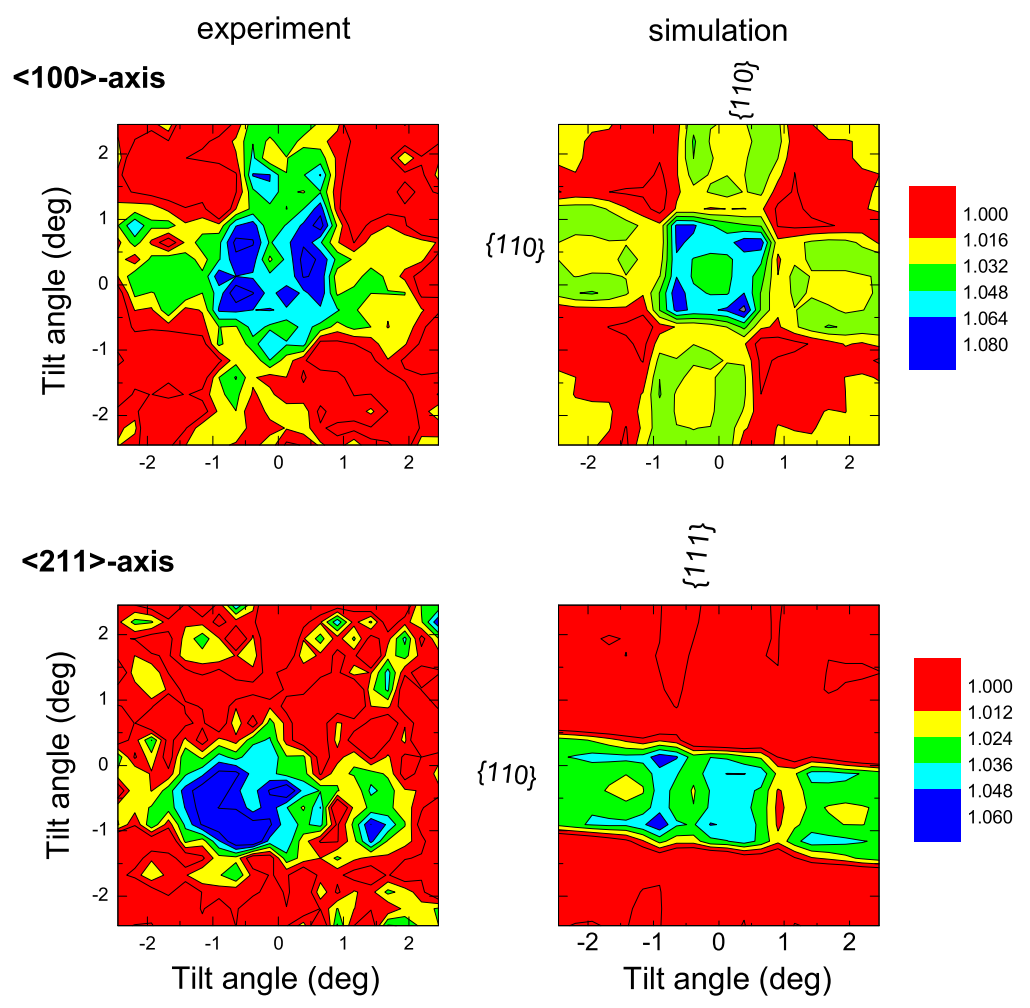

Figure 5.11: Normalized ${ }^{139}$ La conversion electron emission channeling spectra of ${ }^{139} \mathrm{Ce}$ implanted c$\mathrm{BN}$, shown for the $<100>$ as well as the $<211>$ axes. On the right-hand side corresponding simulations are shown. The spectra are taken from ref. [52].

overall agreement between experiment and simulation is moderate, although it can clearly be stated that the lattice location of the implanted $\mathrm{Ce}$ ions is either a near-boron site of very low symmetry, or several sites (including the substitutional boron site) are occupied in the c-BN lattice. Future EC investigations will hopefully shed light on the lattice location of lanthanides in $\mathrm{c}-\mathrm{BN}$.

\subsubsection{Luminescence properties of $\mathrm{Eu}^{3+}$ and $\mathrm{Tm}^{3+}$ in c-BN}

Implantations of stable lanthanide isotopes into poly crystal (pc) and single crystal (sc) c-BN were performed at the ion implantation facility IONAS [12] at an energy of $120 \mathrm{keV}$ and fluences of $1 \cdot 10^{13}$ ions $/ \mathrm{cm}^{2}$. Annealing was performed either under vacuum conditions in a conventional tube furnace or at high temperature and high pressure similar to the conditions under 
which the samples were synthesized. Temperatures as high as $1473 \mathrm{~K}$ were found to be necessary to successfully activate intra- $4 \mathrm{f}$ luminescence of the implanted triply ionized $\mathrm{Eu}^{3+}$ and $\mathrm{Tm}^{3+}$ ions. The best activation was finally achieved under high temperature, high pressure conditions ( $\mathrm{p}=7.7 \mathrm{GPa}, \mathrm{T}=2223 \mathrm{~K}, \mathrm{t}=20 \mathrm{~min}$.), whereby the samples were placed in a $\mathrm{CsCl}$ medium. Subsequent to annealing the samples were rinsed in aqua regia and further cleaned in boiling de-ionized water.

\subsubsection{Cathodoluminescence of $\mathrm{Eu}^{3+}$ in $\mathrm{c}-\mathrm{BN}$}

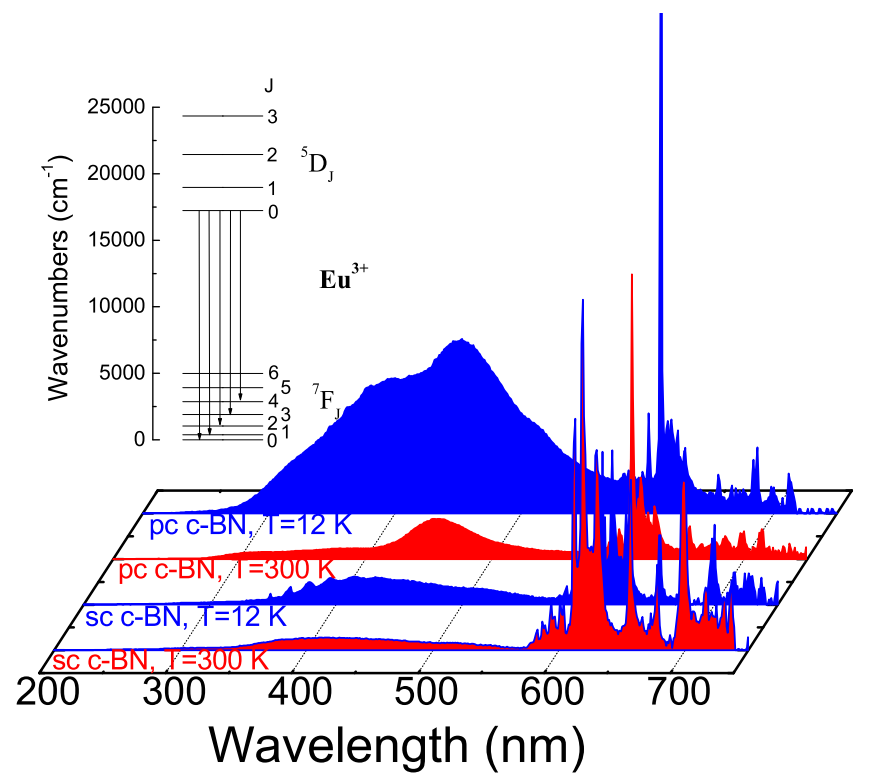

Figure 5.12: Cathodoluminescence survey spectra of $\mathrm{Eu}^{3+}$-implanted polyand single crystalline c-BN, measured at $12 \mathrm{~K}$ and $300 \mathrm{~K}$.

In figure 5.12, $\mathrm{CL}$ spectra for both the Eu-implanted pc and sc c-BN samples are shown. The spectra were recorded at $300 \mathrm{~K}$ and $12 \mathrm{~K}$; an electron energy of $5 \mathrm{keV}$ and a power density of $0.3 \mathrm{~W} / \mathrm{cm}^{2}$ was used. All samples show a more or less pronounced broad intrinsic luminescence of the $\mathrm{c}-\mathrm{BN}$ host in the blue and ultraviolet region, which can be attributed to boron and nitrogen multivacancy complexes [259]. Besides these broad bands, which are more dominant in the $\mathrm{pc}$ c-BN samples, relatively sharp luminescence lines, due to radiative intra- $4 \mathrm{f}$ electron transitions of $\mathrm{Eu}^{3+}$, can be detected. According to the energy level structure of the $\mathrm{Eu}^{3+}\left(4 f^{6}\right)$ electron system and typical transition probabilities for non-centrosymmetric sites, radiative transitions are, within the considered range of the electromagnetic spectrum, expected to occur mainly for transitions starting from the ${ }^{5} D_{0}$ and ${ }^{5} D_{1}$ levels. Based on the observed spectra it can be 
concluded that the ${ }^{5} D_{1}$ level is depopulated non-radiatively, because luminescence due to the ${ }^{5} D_{1} \rightarrow{ }^{7} F_{1}$ transition, typically expected at around $550 \mathrm{~nm}$ [43, 51], is completely absent. Possible explanations are provided by the high phonon energies of the c-BN host which can exceed $1250 \mathrm{~cm}^{-1}$ [260] and the pronounced covalency of the c-BN host [261], which increases the magnitude of the electron-phonon interaction. Therefore all the transitions that were detected were assigned to transitions starting from the ${ }^{5} D_{0}$ level. The luminescence of the pc c-BN sample differs significantly from the sc c-BN specimens, since it shows an additional broad luminescence, centered at around $480 \mathrm{~nm}$, which can be attributed to $4 \mathrm{f}^{N-1} 5 \mathrm{~d} \rightarrow 4 \mathrm{f}^{N}$ transitions of $\mathrm{Eu}^{2+}$ (see e.g. Ref. [55]).

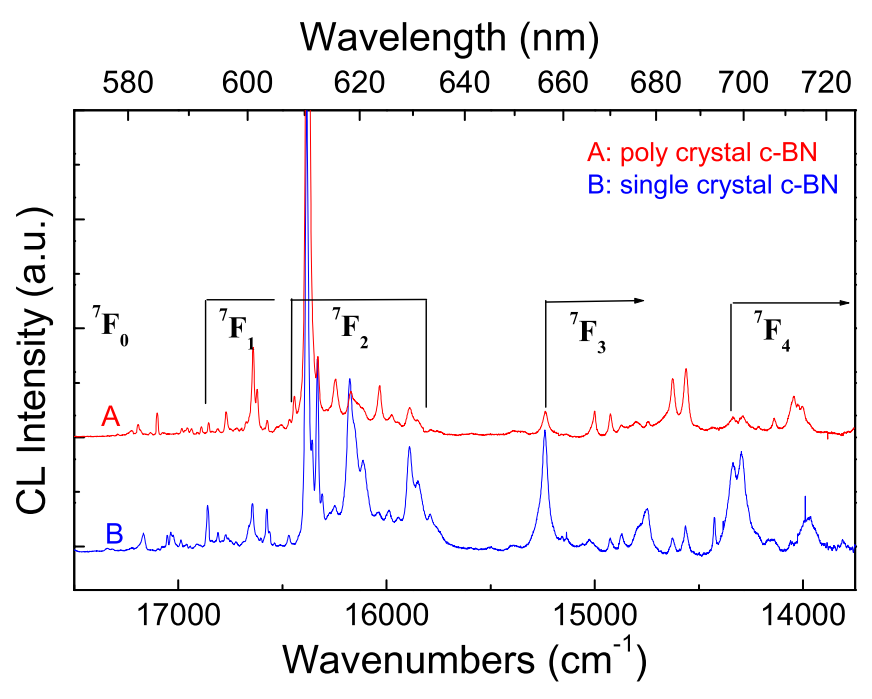

Figure 5.13: High resolution CL spectrum of the ${ }^{5} D_{0} \rightarrow{ }^{7} F_{J}$ transitions of Eu implanted poly- and single crystal c-BN.

In figure 5.13, an enlargement of the $\mathrm{Eu}^{3+}$-related luminescence, recorded at $12 \mathrm{~K}$, is given for both the sc and pc c-BN samples. The most dominant transition ${ }^{5} D_{0} \rightarrow{ }^{7} F_{2}$ shows a very large crystal field splitting of approximately $500 \mathrm{~cm}^{-1}$, similar to values reported for Eu: $\mathrm{Y}_{3} \mathrm{Al}_{5} \mathrm{O}_{12}$, but much larger than the splitting in many other hosts [100]. Comparing both sc and pc crystal samples, many of the energetic positions of the transitions between individual Stark levels overlap, although intensity ratios vary between the two samples. Differing intensities might be an indication of the existence of more than one site of the $\mathrm{Eu}^{3+}$ ions.

Figure 5.14 (upper graph) shows high resolution spectra in the range $605-625 \mathrm{~nm}$ for selected temperatures between $12 \mathrm{~K}$ and $300 \mathrm{~K}$, covering only a part of the ${ }^{5} D_{0} \rightarrow{ }^{7} F_{2}$ transitions. The evolution of the integral intensity of the luminescence in the range $605-625 \mathrm{~nm}$ is shown in figure 5.14 (lower graph), together with the evolution for some selected transitions between 

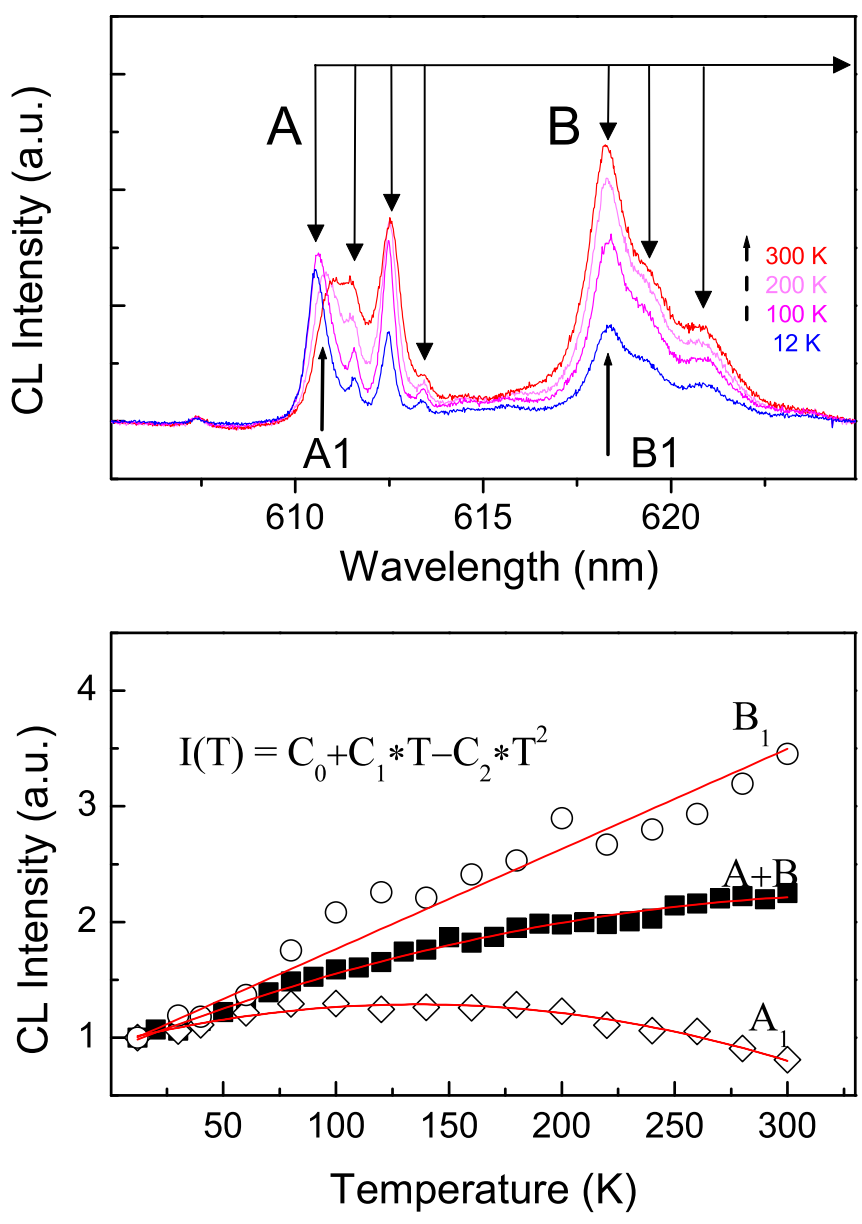

Figure 5.14: Upper graph: high resolution CL spectrum of the ${ }^{5} D_{0} \rightarrow{ }^{7} F_{2}$ transition of $\mathrm{Eu}^{3+}$, implanted into poly crystal c-BN, measured at different temperatures. Lower graph: temperature dependent intensity of the luminesence in the range $605-625 \mathrm{~nm}$.

individual Stark levels (lines A1 and B1). We find that the evolution of the intensity $I(T)$ with increasing temperature $T$ is well described by the formula

$$
I(T)=C_{0}+C_{1} \cdot T-C_{2} \cdot T^{2}
$$

where $C_{0,1,2}$ are constants, which vary between different transitions. Assuming temperatureindependent branching ratios, the observed behavior is another evidence for several sites of the $\mathrm{Eu}^{3+}$ ions in the c-BN host.

Finally, lifetime measurements of the transitions at $612 \mathrm{~nm}$ (group A) were performed. The decay of the luminescence was found to be reasonably described by a double exponential decay with two independent mean lifetimes of around $381 \mu \mathrm{s}$ and $132 \mu \mathrm{s}$. As already mentioned, the assumption of a second site is reasonable. 


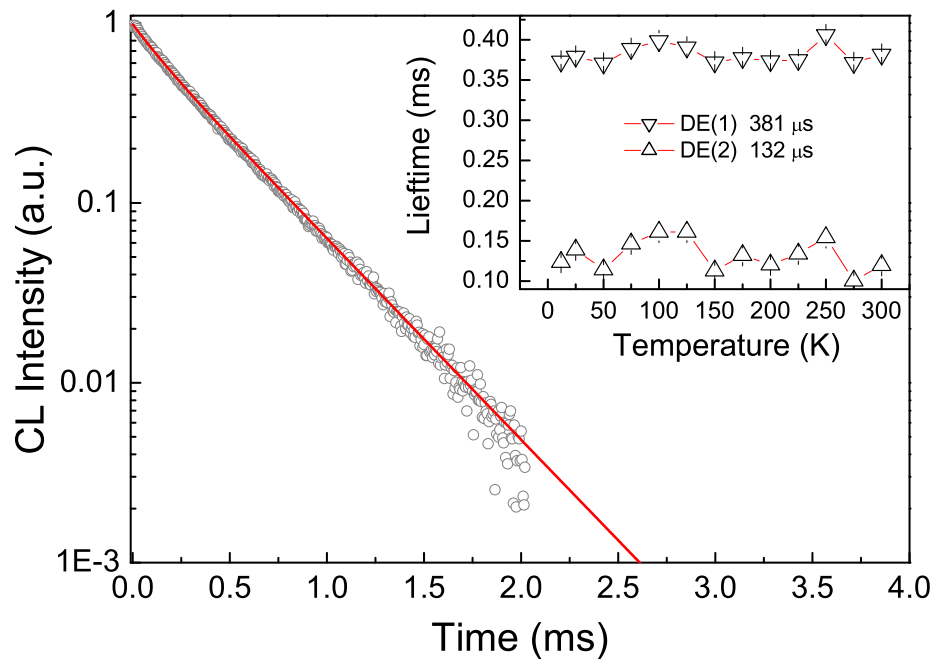

Figure 5.15: Luminescence decay of the ${ }^{5} D_{0}$ level of $\mathrm{Eu}^{3+}$ in c-BN, monitored at $611 \mathrm{~nm}\left({ }^{5} D_{0} \rightarrow{ }^{7} F_{2}\right)$.

\subsubsection{Cathodoluminescence of $\mathrm{Tm}^{3+}$ in $\mathrm{c}-\mathrm{BN}$}

Doping of poly crystalline c-BN with Tm by ion implantation was achieved under the same experimental conditions as described above for the case of Eu in the poly crystal c-BN. In Figure 5.16, the survey scan, measured at $300 \mathrm{~K}$ as well as $12 \mathrm{~K}$, is shown. Several luminescence lines related to radiative intra- $4 \mathrm{f}$ transition of $\mathrm{Tm}^{3+}$ were detected. The assignment of these lines to transitions between $L S J$ multiplets is tentative, and it is based on energy differences among the free ion energy levels, except the ${ }^{1} I_{6} \rightarrow{ }^{3} H_{6}$ transition which does not overlap with any other transition. Potentially overlapping transitions are ${ }^{1} I_{6} \rightarrow{ }^{3} F_{4}$ and ${ }^{1} D_{2} \rightarrow{ }^{3} H_{6}$ as well as ${ }^{1} I_{6} \rightarrow{ }^{3} F_{5},{ }^{1} D_{2} \rightarrow{ }^{3} F_{4}$ and ${ }^{1} G_{4} \rightarrow{ }^{3} H_{6}$. In principle, these transitions could be further distinguished by time-resolved spectroscopy but because of the long-lived defect luminescence which is the dominant underlying background in all cases, these measurements were not performed. In contrast to the case of $\mathrm{Eu}^{3+}$ in c-BN, the $\mathrm{Tm}^{3+}$-related luminescence in c-BN is much weaker with repect to the ratio of defect luminescence of the $\mathrm{c}-\mathrm{BN}$ host than the radiative intra- $4 \mathrm{f}$ electron transitions of the lanthanide ions. Concerning the energetic position, a remarkable red shift of the $\mathrm{Tm}^{3+}$-related luminescence bands can be seen, which again points to a very large crystal field splitting similar to that in the case of $\mathrm{Eu}^{3+}$ in c-BN.

Figure 5.17 shows the temperature dependent luminescence of both the ${ }^{1} G_{4} \rightarrow{ }^{3} H_{6}$ and ${ }^{1} D_{2} \rightarrow{ }^{3} F_{4}$ transitions of $\mathrm{Tm}^{3+}$ in c-BN. The overall temperature behavior which shows thermal quenching, is in contrast to the behavior observed for $\mathrm{Eu}^{3+}$ in $\mathrm{c}-\mathrm{BN}$ where the luminescence of the strongest transition ${ }^{5} D_{0} \rightarrow{ }^{7} F_{2}$ is increasing with increasing temperature. 


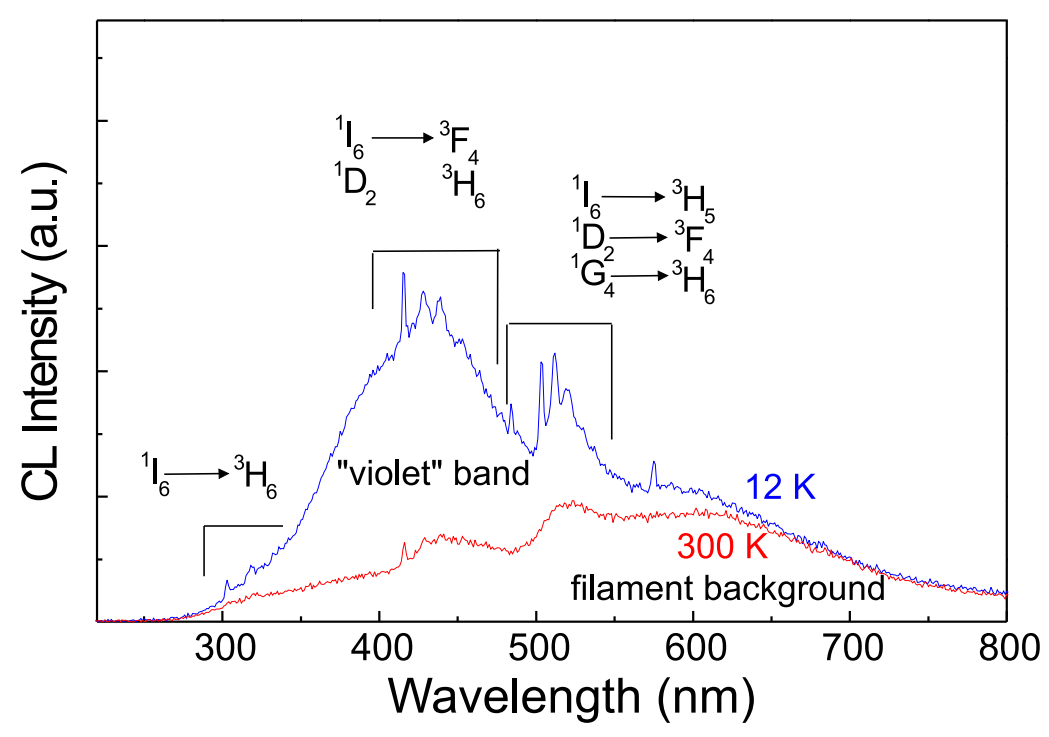

Figure 5.16: CL survey spectra of $\mathrm{Tm}^{3+}$-implanted poly crystal and single crystal c-BN, measured at $12 \mathrm{~K}$ and $300 \mathrm{~K}$.

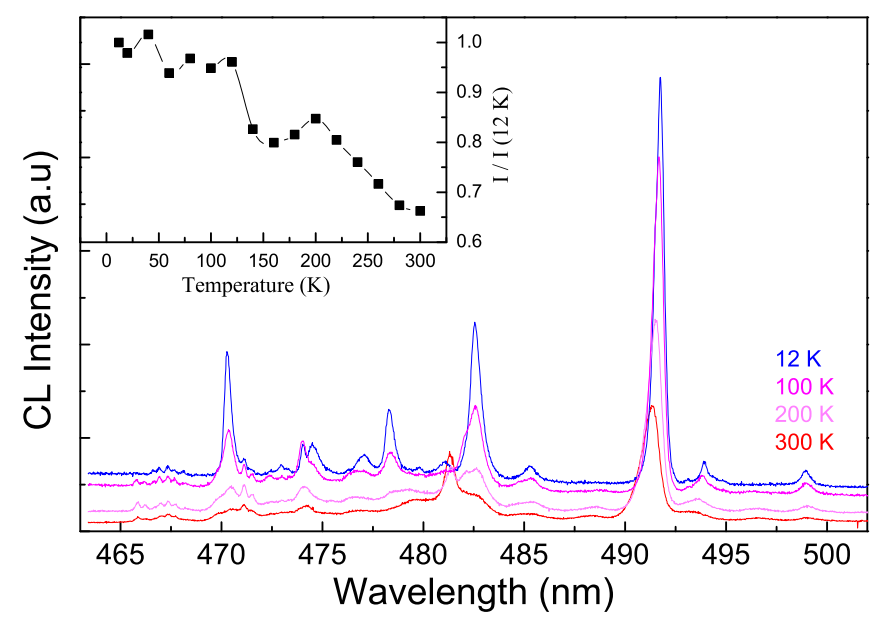

Figure 5.17: Temperature dependent high resolution $\mathrm{CL}$ spectra of the ${ }^{1} G_{4} \rightarrow{ }^{3} H_{6}$ and ${ }^{1} D_{2} \rightarrow{ }^{3} F_{4}$ transitions of $\mathrm{Tm}^{3+}$ in c-BN. A linear background was subtracted and the spectra are shifted for better illustration.

\subsubsection{Summary}

Conversion electron emission channeling studies of ${ }^{139} \mathrm{Ce}$ ions, implanted into c-BN suggest that the implanted ions are either located on near-substitutional boron sites or occupy different sites in the c-BN host. The lattice location studies, performed after annealing the implanted samples to $1173 \mathrm{~K}$, revealed only a small fraction of the implanted ions on these sites. This suggests that much higher annealing temperatures are necessary to anneal the defects in the cBN host after ion implantation. Further investigations are necessary in order to unambiguously identify the lattice sites of lanthanides in $\mathrm{c}-\mathrm{BN}$. 


\subsection{CUBIC BORON NITRIDE}

Europium-implanted c-BN, annealed under high temperature and high pressure conditions, clearly shows luminescence due to the ${ }^{5} D_{0} \rightarrow{ }^{7} F_{2}$ intra- $4 f$ electron transition of $\mathrm{Eu}^{3+}$. Additionally, in the poly crystal c-BN specimens luminescence arising from $4 f^{N-1} 5 d \rightarrow 4 f^{N}$ electron transition of $\mathrm{Eu}^{3+}$ is visible. Luminescence involving the upper initial ${ }^{5} D_{1}$ level were found to be absent. This was attributed to high optical phonon frequencies and the pronounced covalency of the $\mathrm{c}-\mathrm{BN}$ host. With increasing temperature an increase in the integral intensity of the ${ }^{5} D_{0} \rightarrow{ }^{7} F_{2}$ transitions was found.

Thulium-implanted $\mathrm{c}-\mathrm{BN}$, annealed under high temperature and high pressure conditions, shows only moderate luminescence related to intra- $4 f$ electron transitions of $\mathrm{Tm}^{3+}$. With increasing temperature, a decrease in the integral intensity of dominant $\mathrm{Tm}^{3+}$-related luminescence could be seen. 


\subsection{Silicon carbide}

Silicon carbide has become the material of choice for the implementation of high power high temperature devices, which is due to its good thermal and electrical properties and the possibility of synthesizing SiC bulk specimens [262, 246, 263, 264]. These are often used as substrates for the growth of other wide band gap semiconductor thin films, such as GaN and AlN. SiC features a large number of polytypes, of which the most frequently used ones are $6 \mathrm{H}-\mathrm{SiC}, 4 \mathrm{H}-\mathrm{SiC}$ and $3 \mathrm{C}-\mathrm{SiC}$. The most important physical properties of these polytypes are summarized in table A.3. Low-temperature optical characterization of the $6 \mathrm{H}-, 4 \mathrm{H}-$, and $3 \mathrm{C}-$ $\mathrm{SiC}$ polytypes can be found in the work of Devaty and Choyke [265] and Pensl and Choyke [266]. Several authors studied the annealing behavior of defects generated upon implantation (see e.g. [267, 268, 269, 270]) and it is an accepted fact that such defects can only be completely annealed at high temperatures.

Up to now, the luminescence behaviour of $\mathrm{SiC}$ doped with lanthanides has been almost exclusively studied for $\mathrm{Er}^{3+}$, either doped into $\mathrm{SiC}$ during growth [76, 28, 29] or by ion implantation [27, 271]. Choyke et al. reported $\mathrm{Er}^{3+}$-related photoluminescence in several ion-implanted $\mathrm{SiC}$ polytypes for temperatures up to $525 \mathrm{~K}$. The best activation of $\mathrm{Er}^{3+}$-related luminescence was achieved after annealing the $\mathrm{SiC}$ specimens at $1973 \mathrm{~K}$ in a SiC cavity filled with pure argon. Kozanecki et al. reported room-temperature luminescence of $\mathrm{Er}^{3+}$-implanted $6 \mathrm{H}-\mathrm{SiC}$ which was annealed at temperatures below $1623 \mathrm{~K}$. It is believed that erbium doped into SiC either during growth or by ion implantation takes substitutional silicon lattice sites. In this case, $\mathrm{Er}^{3+}$ will behave non-isoelectronically and the creation of defects for charge compensation is likely. Indeed, Baranov et al. reported four different local environments, based on EPR measurements of $\mathrm{Er}^{3+}$ doped into SiC during growth. Three of these centers are of low symmetry due to carbon vacancies $V_{C}$ in the neighborhood of the $\mathrm{Er}^{3+}$ ions. The situation in the $6 \mathrm{H}-\mathrm{SiC}$ polytype is complicated because two different substitutional silicon sites exist, one of them being cubic and the other one hexagonal, which can be seen from the alignment of the third nearest ligands. These observations suggest that a crystal field analysis is very complicated and would have to be based on time-resolved measurements. Finally, the work of Pietsch et al. who doped 3C-SiC with neodymium during growth, is worth mentioning. The authors report that no luminescence related to intra-4f electron transitions of $\mathrm{Nd}^{3+}$ could be found.

The most important physical properties of the 3C-, 4H-, and 6-SiC polytpes, as far as this work is concerned, are summarized in table A.3, the unit cells are shown in Figure A.1. The lattice constants and positions of the $\mathrm{Si}$ and $\mathrm{C}$ atoms in the unit cell are input parameters for the emission channeling investigation reported in the following chapter. 


\subsubsection{Lattice location of ${ }^{169} \mathbf{T m}$ in $3 \mathrm{C}-\mathrm{SiC},{ }^{167} \mathbf{T m}$ in $\mathbf{4 H - S i C}$ and ${ }^{169} \mathbf{Y b}$ in 6H-SiC}

As mentioned above, lanthanides implanted into SiC are believed to take substitutional Si sites. In order to directly determine the lattice site of lanthanides implanted into $\mathrm{SiC}$, emission channeling measurements were performed, which will be discussed in the following.

Radioactive lanthanide ions were implanted at the on-line isotope separator ISOLDE at CERN [221] with an energy of $60 \mathrm{keV}$ upon irradiation of a tantalum target with energetic protons, surface ionization and mass separation. Ions of mass 167 and 169 were implanted to a fluence of $2 \cdot 10^{13}$ ions $/ \mathrm{cm}^{2}$ within 30 minutes, thus yielding an ion flux of approx. $10^{10} \mathrm{ions} / \mathrm{cm}^{2} \mathrm{~s}$. In this case, the implanted isobars are known to be ${ }^{167} \mathrm{Yb}$ and ${ }^{169} \mathrm{Lu}$, which is determined by means of $\gamma$-spectroscopy. Thus the $3 \mathrm{C}$ - and $4 \mathrm{H}-\mathrm{SiC}$ polytypes were implanted with ${ }^{167} \mathrm{Yb}$ while the
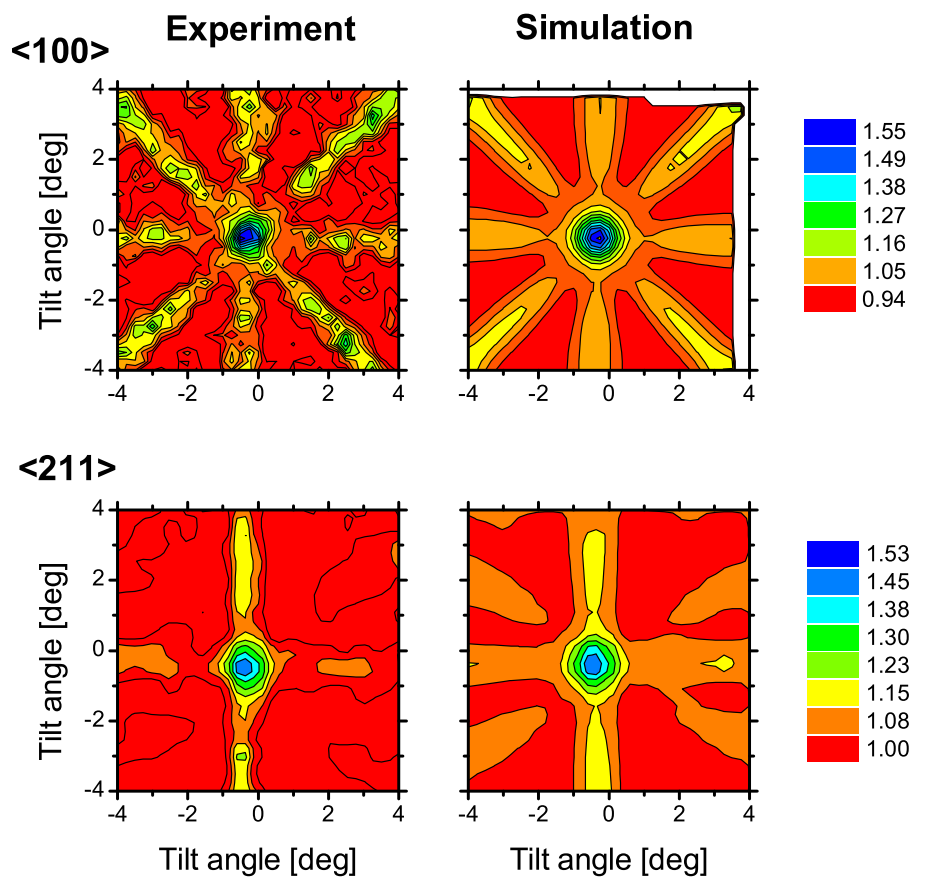

Figure 5.18: Normalized 2-dimensional channeling spectra of conversion electrons from ${ }^{167} \mathrm{Er}$ in $3 \mathrm{C}-$ $\mathrm{SiC}$ as implanted, for the $\langle 110\rangle$ and $\langle 211\rangle$ axial directions. The best fits to the experimental spectra correspond to a fraction of 46(9) \% of emitter atoms on substitutional $\mathrm{Si}$, with a mean static displacement of $0.2 \AA$. 


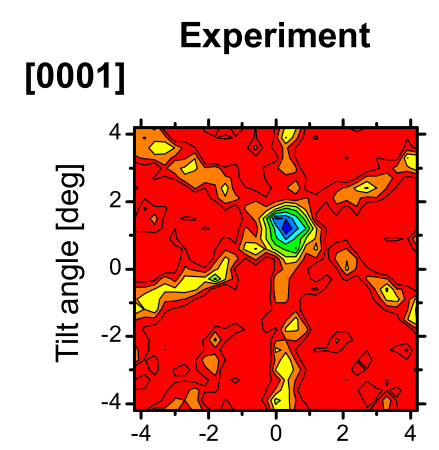

\section{Simulation}

[11르]
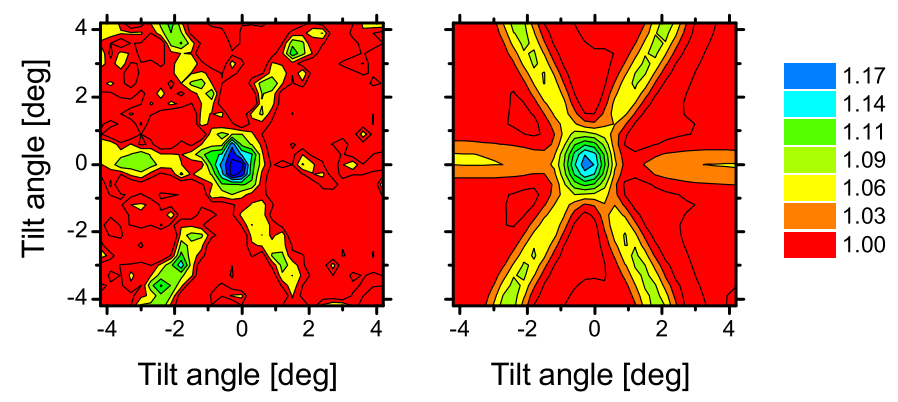

Figure 5.19: Normalized 2-dimensional channeling spectra of conversion electrons from ${ }^{167} \mathrm{Er}$ in $4 \mathrm{H}-$

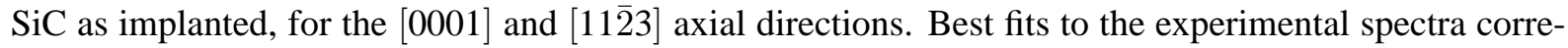
spond to a fraction of 58(5) \% of emitter atoms on substitutional silicon sites with a mean static displacement of $0.25 \AA$.

$6 \mathrm{H}-\mathrm{SiC}$ polytype was implanted with ${ }^{169} \mathrm{Lu}$. The corresponding decay chains and experimental energy spectra are presented in chapter 3.2 .5 .

The electrons used for the EC measurements were the $150 \mathrm{keV}$ and $198 \mathrm{keV}$ conversion electrons of ${ }^{167} \mathrm{Er}$, and $118 \mathrm{keV}$ and $138 \mathrm{keV}$ conversion electrons of ${ }^{169} \mathrm{Tm}$, respectively. Isochronal annealing (10 $\mathrm{min})$ of the samples was performed in vacuo without dismounting the samples from the goniometer. The limit for the annealing temperatures was determined by the activities of the samples. According to ref. [272], a mean displacement of $0.057 \AA$ (0.051 $\AA$ ) C (Si) atoms from their ideal lattice site at room temperature is assumed. The depth distributions of the implanted ions were calculated using TRIM [191] by assuming a density of $3.21 \mathrm{~g} / \mathrm{cm}^{2}$ for all SiC polytypes yielding $222 \AA$ (50 $\mathrm{\AA}$ ) as the mean ion range (variance) for ${ }^{169}$ Lu and $226 \AA$ (50 $\mathrm{\AA}$ ) for ${ }^{167} \mathrm{Yb}$ respectively. The simulated patterns were fitted against the normalized experimental spectra using least-squares fitting, as described in Ref. [177] 

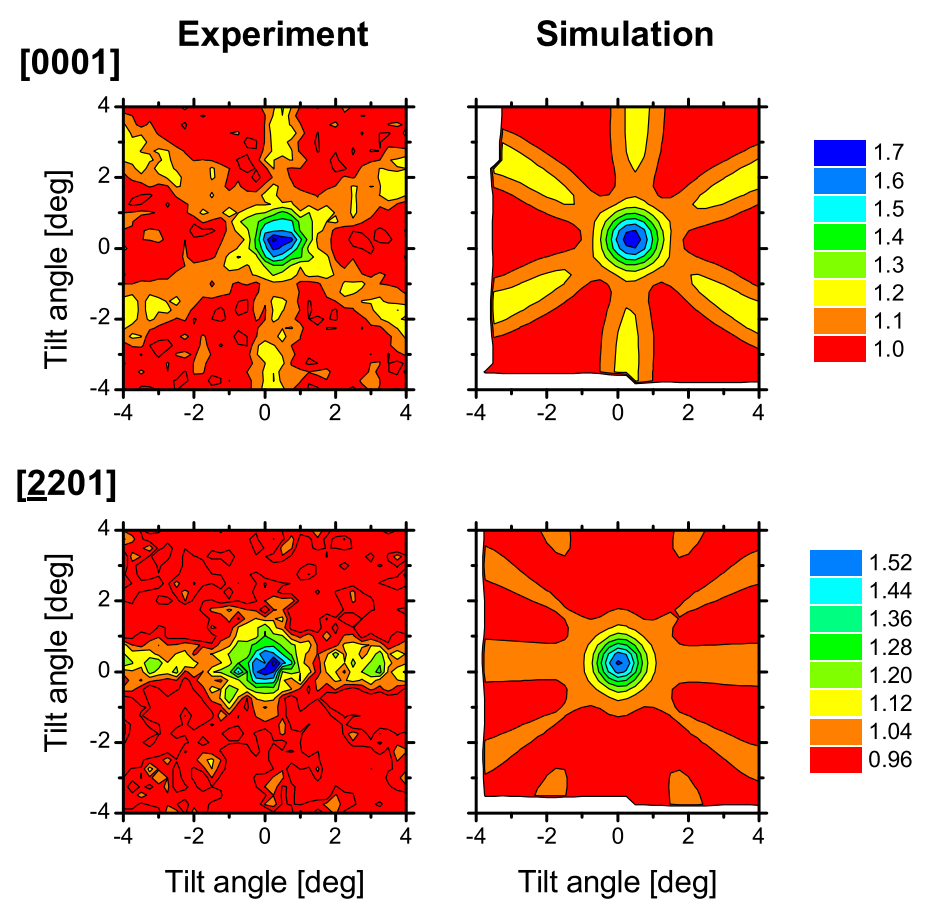

Figure 5.20: Normalized 2-dimensional channeling spectra of conversion electrons from ${ }^{169} \mathrm{Tm}$ in $6 \mathrm{H}-$ $\mathrm{SiC}$ annealed at $1073 \mathrm{~K}$, for the [0001] and [2201] axial directions. The best fits are obtained when assuming 92(5) \% of emitter atoms on substitutional $\mathrm{Si}$ sites for the [0001] axis as well as 51(5) \% for the $[\overline{2} 201]$ axis, with a mean static displacement of $0.2 \AA$.

Fig. 5.3.1 shows the measured EC spectra of the conversion electrons from ${ }^{167} \mathrm{Er}$ in $3 \mathrm{C}-\mathrm{SiC}$, as implanted, with respect to the tilt angle for the $\langle 100\rangle$ and $\langle 211\rangle$ axis. The angular resolution of the experimental setup was $0.34^{\circ}$. The intensities of the conversion electrons of ${ }^{167} \mathrm{Er}$ are normalized with respect to a random orientation. The best fits were obtained for assuming $46(9) \%$ of emitter atoms on substitutional Si sites, with a mean static displacement of $0.2 \AA$. The remaining atoms were considered to be located on "random" sites.

Fig. 5.19 shows the normalized EC spectra of conversion electrons from ${ }^{167} \mathrm{Er}$ in $4 \mathrm{H}-\mathrm{SiC}$, as implanted, with respect to the tilt angle for the [0001] and [11 $\overline{2} 3]$ axes. The angular resolution of the setup was $0.4^{\circ}$. The best fits were obtained when assuming 58(5) \% of emitter atoms take on substitutional Si sites, with a mean static displacement of $0.25 \AA$ and the remainder on "random" sites.

EC measurements of the implanted $6 \mathrm{H}-\mathrm{SiC}$ sample showed only very weak channeling patterns, 


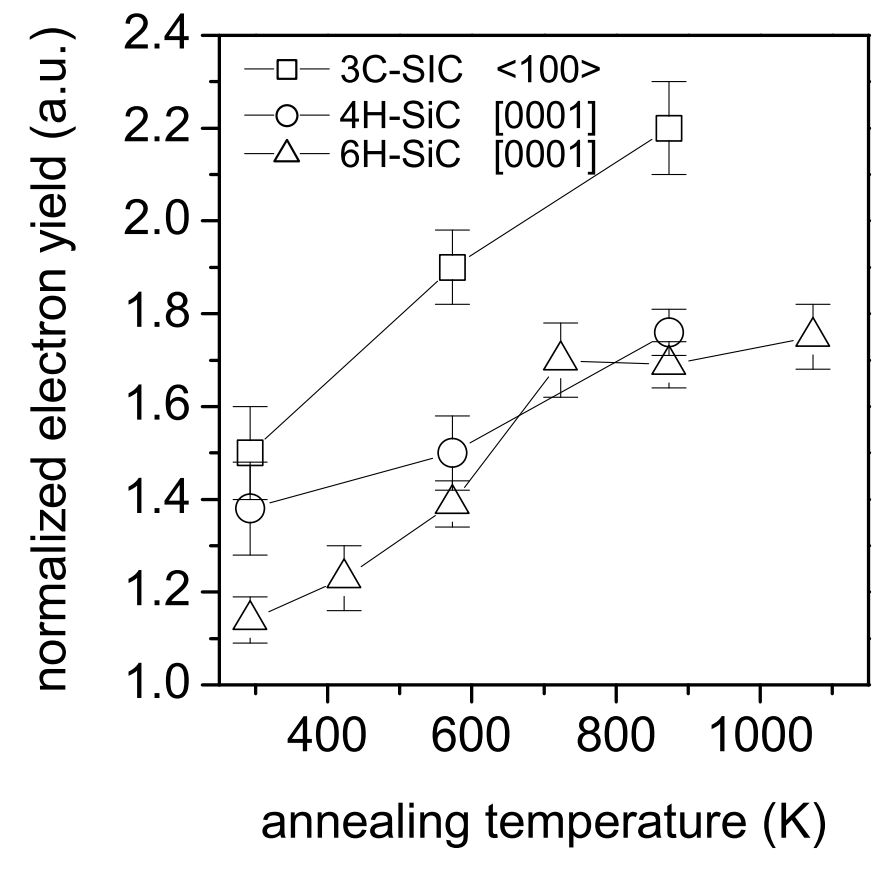

Figure 5.21: Dependence of the normalized conversion electron yields of ${ }^{167} \mathrm{Tm}$ and ${ }^{169} \mathrm{Yb}$ on the annealing temperature for the $3 \mathrm{C}-, 4 \mathrm{H}-$ and $6 \mathrm{H}-\mathrm{SiC}$ polytpes. The axes, to which the normalized electron yields refer, are indicated.

which improved considerably upon annealing. Normalized EC spectra of conversion electrons from ${ }^{169} \mathrm{Tm}$ in $6 \mathrm{H}-\mathrm{SiC}$ after annealing the sample at $1073 \mathrm{~K}$ are shown in fig. 5.20. The angular resolution of the setup was $0.39^{\circ}$. The spectra were recorded around the [0001] and [2201] axes. The best fits were obtained when assuming 92(5) \% of emitter atoms on substitutional Si sites for the $[0001]$ axis as well as 51(5) \% for the [2201] axis, with a mean static displacement of $0.2 \AA$. This large discrepancy between the two directions has to be attributed to the oversimplified assumption of ideal substitutional silicon sites. However, such a displacement from the ideal silicon site is expected to be in the range of $\sim 0.2-0.3 \AA$ in this case. It should be noted that the assumed mean static displacement may have several reasons. First of all, the local surrounding of the emitters may be heavily damaged upon implantation, thus leading to displaced emitter atoms. Secondly, with respect to the EPR measurements of Baranov et al. [76] and the PAC investigations of Achtziger et al. [268] mentioned earlier, a directed displacement due to lanthanide-carbon vacancy interaction is feasible as well.

Finally, the evolution of the normalized electron yields is shown for the systems mentioned above. This was done by additionally recording one-dimensional scans around the same axes as before. Figure 5.21 shows the normalized conversion electron yields from ${ }^{167} \mathrm{Er}$ and ${ }^{169} \mathrm{Tm}$ within the $\mathrm{SiC}$ polytypes as described above. The axes, to which the normalized electron yields refer, are indicated in the figure. Although one-dimensional scans are ambiguous with respect 


\subsection{SILICON CARBIDE}

to the determination of fractions of emitter atoms on certain sites, they clearly indicate whether a change occurs upon annealing or not. As the normalized electron yields increase with increasing annealing temperature, more emitter atoms are taking up substitutional Si sites. Thus, the observed behavior is different to that of III-V semiconductors like GaN and AlN (see this work), where implanted lanthanide ions, to a large extent, occupy isoelectronic substitutional $\mathrm{Ga}$ or Al sites after implantation, and where this fraction stays constant upon annealing [181]. 


\subsection{2 $\mathbf{T m}^{3+}$ doped $6 \mathbf{H}-\mathrm{SiC}$ - infrared light emission at $805 \mathrm{~nm}$}

To investigate the cathodoluminescence of Tm-implanted $6 \mathrm{H}-\mathrm{SiC}$, both the samples used for emission channeling experiments and separately implanted samples were used. The additional samples were made by implanting ${ }^{169} \mathrm{Tm}^{+}$ions at a fluence of $1 \cdot 10^{13}$ ions $/ \mathrm{cm}^{2}$ and energies of $100 \mathrm{keV}$ into commercially available substrates (see table A.2). Annealing of the samples was performed under vacuum conditions at a temperature o $1473 \mathrm{~K}$. Each sample was covered with a second sample to reduce segregation of carbon.

With regard to the band gap of the $6 \mathrm{H}-\mathrm{SiC}$ host of approximately $3.0 \mathrm{eV}$ (see table A.3) in comparison to the energy level diagram of $\mathrm{Tm}^{3+}$ shown in figure 3.2 , it is obvious that only transitions from the ${ }^{1} G_{4}$ level and other levels energetically located below are possible. This is similar to the case of GaN [26, 41, 42, 195]. In figure 5.22, the CL spectra of the ${ }^{1} G_{4} \rightarrow{ }^{3} H_{6}$ intra-4f electron transition of $\mathrm{Tm}^{3+}$ in $6 \mathrm{H}-\mathrm{SiC}$ are shown. The sample was used for emission channeling measurements as described in section 5.3.1, and was therefore annealed at $1073 \mathrm{~K}$. The ${ }^{1} G_{4} \rightarrow{ }^{3} H_{6}$ transition shows a large number of mostly very sharp lines. The overall number of individual lines is too large to account for only one site of the $\mathrm{Tm}^{3+}$ ions. With increasing temperature the luminescence corresponding to the ${ }^{1} G_{4} \rightarrow{ }^{3} H_{6}$ transition is more and more quenched and is completely absent at $160 \mathrm{~K}$. To understand this behaviour time-resolved CL spectroscopy was performed on the arbitrary line (A) shown in figure 5.22. The decay curve of the luminescence from the ${ }^{1} G_{4} \rightarrow{ }^{3} H_{6}$ transition is shown in figure 5.23 . At $12 \mathrm{~K}$, the decay is almost exponential while at $50 \mathrm{~K}$ a non-exponential decay can be found. It is obvious that the lifetime is strongly reduced being an indication that the thermal quenching which was shown in Figure 5.22 is due to a non-radiative backtransfer process rather than solely related to the

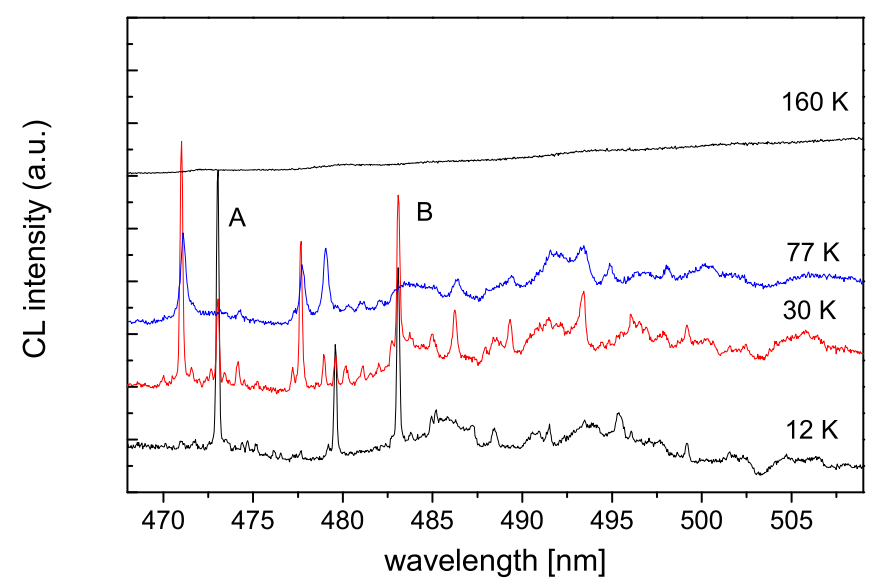

Figure 5.22: ${ }^{1} G_{4} \rightarrow{ }^{3} H_{6}$ intra-4f electron transition of $\mathrm{Tm}^{3+}$ in $6 \mathrm{H}-\mathrm{SiC}$, measured at different temperatures. Besides very sharp transitions, a large number of less pronounced weak transition can be seen. At $160 \mathrm{~K}$, no luminescence related to $\mathrm{Tm}^{3+}$ is visible, which is attributed to thermal quenching. 


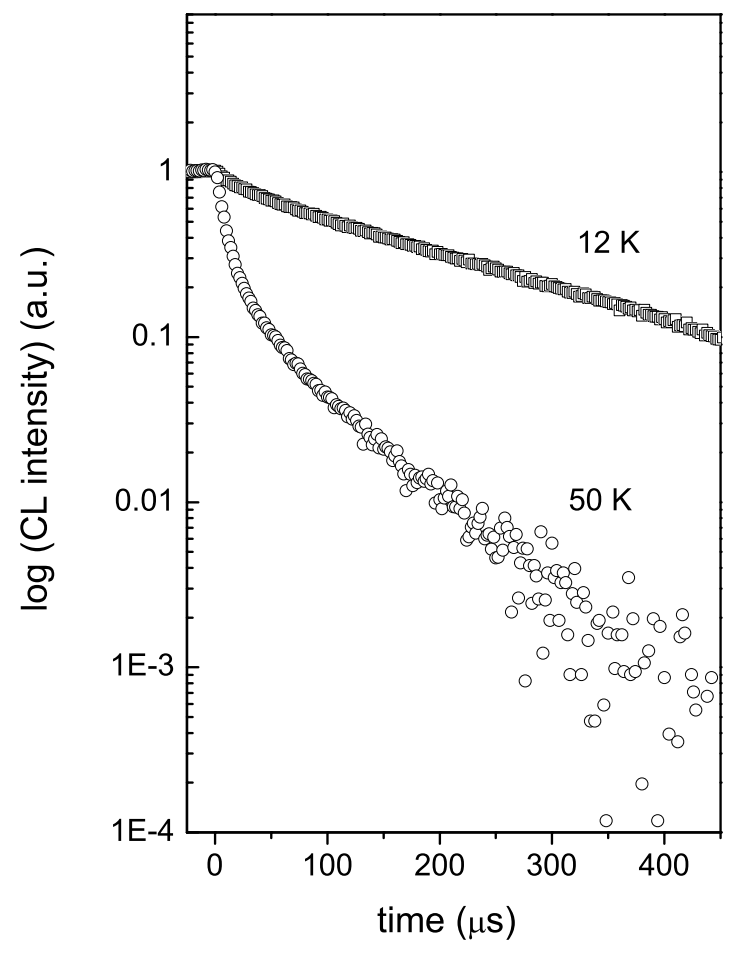

Figure 5.23: Time resolved $\mathrm{CL}$ spectra of the ${ }^{1} G_{4} \rightarrow{ }^{3} H_{6}$ transition of $\mathrm{Tm}^{3+}$ in $6 \mathrm{H}-\mathrm{SiC}$, monitored at $482.2 \mathrm{~nm}$. The decay at $12 \mathrm{~K}$ is approximately exponential while at $50 \mathrm{~K}$ a non-exponential behaviour is found. Thermal quenching is clearly visible in the $50 \mathrm{~K}$ spectrum.

excitation mechanism.

In order to further increase the annealing temperatures of Tm-implanted $\mathrm{SiC}$, a commericially available substrate was used; the sample preparation was discussed before. As already mentioned above, high temperature annealing with temperatures up to $1973 \mathrm{~K}$ was applied by several authors in the case of $\mathrm{Er}^{3+}$-implanted $6 \mathrm{H}-\mathrm{SiC}$. However, the maximum available annealing temperature was $1473 \mathrm{~K}$ and therefore the implanted $\mathrm{SiC}$ samples were annealed at $1473 \mathrm{~K}$ under vacuum conditions $\left(10^{-6} \mathrm{mbar}\right)$ for half an hour. The CL survey scan, recorded at $12 \mathrm{~K}$ as well as $300 \mathrm{~K}$ is shown in Figure 5.24. Besides luminescence arising from free and bound excitons in the $6 \mathrm{H}-\mathrm{SiC}$ host [265], the spectrum at $12 \mathrm{~K}$ is dominated by a green luminescence band arising from defects accompanied by sharp lines which can be attributed to the shell structure of donor-acceptor-pairs [265] as well as the ${ }^{1} G_{4} \rightarrow{ }^{3} H_{6}$ transition. At around $805 \mathrm{~nm}$ the ${ }^{3} H_{4} \rightarrow{ }^{3} H_{6}$ transition can be found additionally. This tentative assignment is based on the fact that at $300 \mathrm{~K}$ the ${ }^{1} G_{4} \rightarrow{ }^{3} H_{6}$ transition is not found at $300 \mathrm{~K}$, and seems to be thermally quenched as discussed above. Figure 5.25 shows the corresponding Stark level CL spectra recorded with the CCD in the range of $790-820 \mathrm{~nm}$ for selected measurement temperatures. Again the low temperature spectrum recorded at $12 \mathrm{~K}$ shows very sharp lines, which are related to transitions between individual Stark levels within $\mathrm{Tm}^{3+}$. With increasing temperature, the thermal popula- 


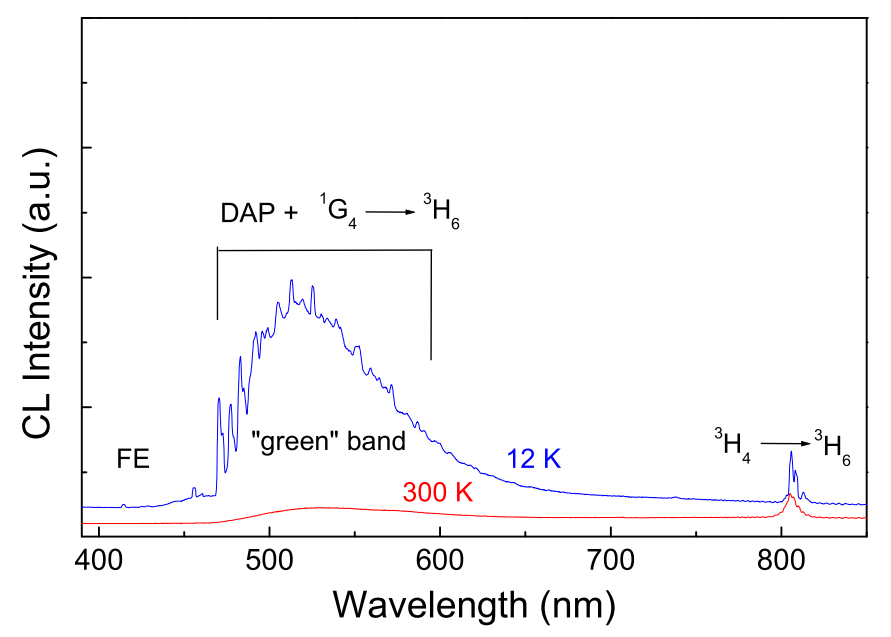

Figure 5.24: Cathodoluminescence survey scan of $\mathrm{Tm}^{3+}$-implanted $6 \mathrm{H}$ $\mathrm{SiC}$, measured at $12 \mathrm{~K}$ as well as $300 \mathrm{~K}$.

tion of higher lying levels of the initial multiplet manifolds, leading to an additional change in lifetime, as well as thermal quenching of the integral intensity, can be observed. An important result is that the integral intensity at $300 \mathrm{~K}$ still amounts to $45 \%$ of the one at $12 \mathrm{~K}$ and therefore room-temperature $\mathrm{CL}$ as been successfully realized. For the time being some questions, related to deficiencies of the experimental setup, remain unanswered. From the spectra in Figure 5.25 it doesn't become clear how many local environments are present. In principle, this could only be answered by applying time-resolved spectroscopy. However, measuring each transition separately with the setup described earlier would be very time-consuming, and such measurements should be taken using a CCD camera. Regarding the annealing conditions, it is likely that the optimal activation of $\mathrm{Tm}^{3+}$-related luminescence has not yet been reached.

\subsubsection{Summary}

It was shown that lanthanide ions $\mathrm{Yb}$ and $\mathrm{Tm}$, implanted into $3 \mathrm{C}-, 4 \mathrm{H}-$ and $6 \mathrm{H}-\mathrm{SiC}$ occupy near-substitutional silicon sites. For Tm in $3 \mathrm{C}-$ and $4 \mathrm{H}-\mathrm{SiC}$ this was shown with the samples as implanted. For $\mathrm{Tm}$ in $3 \mathrm{C}-\mathrm{SiC}$ a fraction of $46(9) \%$ of the implanted ions was found to be located on substitutional silicon sites upon implantation. For $\mathrm{Tm}$ in $4 \mathrm{H}-\mathrm{SiC}$ a fraction of $58(5) \%$ of the implanted ions was found to be located on substitutional silicon sites upon implantation. For $\mathrm{Yb}$ in $6 \mathrm{H}-\mathrm{SiC}$, investigated after annealing at $1073 \mathrm{~K}$, a discrepancy in fitting the fraction of ions against substitutional silicon sites is found for different axes indicating that this assumption of the site is oversimplified. Although it is likely that almost all implanted $\mathrm{Yb}$ ions are located on near-substitutional silicon sites after annealing at $1073 \mathrm{~K}$, this has to be proven by means of future investigations. Finally, it has been shown that annealing of the 


\subsection{SILICON CARBIDE}

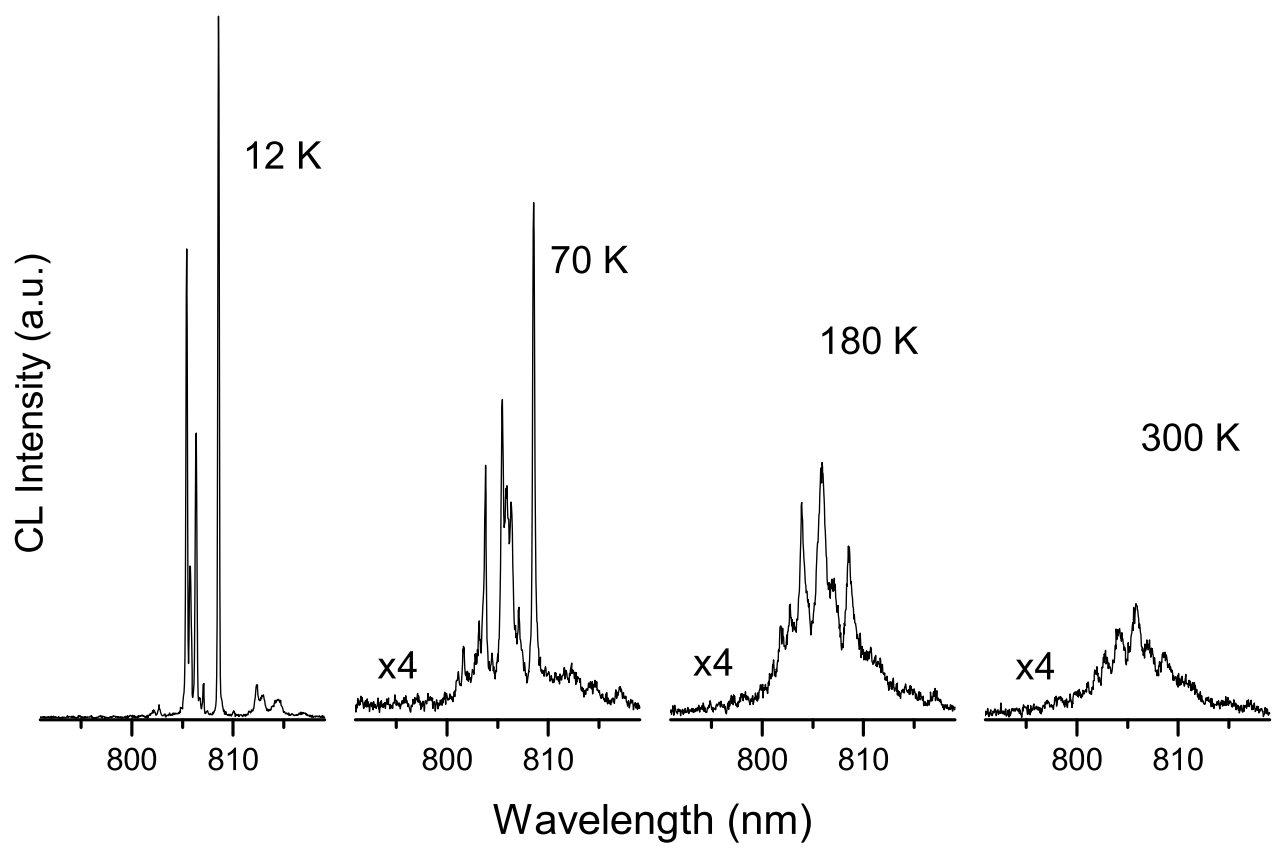

Figure 5.25: ${ }^{3} \mathrm{H}_{4} \rightarrow{ }^{3} \mathrm{H}_{6}$ intra-4f electron transition of $\mathrm{Tm}^{3+}$ in $6 \mathrm{H}-\mathrm{SiC}$, measured at different temperatures. The sample was annealed at $1473 \mathrm{~K}$ under vacuum conditions for 30 minutes. The integral intensity at $300 \mathrm{~K}$ still amounts to $45 \%$ of the intensity at $12 \mathrm{~K}$.

samples leads to an increase in the fraction of the implanted ions on substitutional lattice sites in all three $\mathrm{SiC}$ polytypes which is in contrast to the behavior of lanthanide impurities in III-V semiconductors like GaN.

Cathodoluminescence of thulium implanted $6 \mathrm{H}-\mathrm{SiC}$ specimens, annealed under vacuum conditions and a temperature of $1473 \mathrm{~K}$, has revealed room-temperature infrared luminescence around $805 \mathrm{~nm}$. This luminescence can be related to radiative ${ }^{3} \mathrm{H}_{4} \rightarrow{ }^{3} \mathrm{H}_{6}$ intra-4f electron transitions of $\mathrm{Tm}^{3+}$. Transitions starting from the ${ }^{1} G_{4}$ level seem to be absent at higher temperatures due to thermal quenching. 


\subsection{Diamond}

Diamond is the most covalent and hardest material currently known. The most important parameters of diamond, with regard to this work, are summarized in table A.3. Because the prospects for ion implanted diamond as a light emitter were once thought to be very promising, a reasonable number of publications on the annealing behavior of diamond after ion irradiation is available (see e.g. refs. [273, 274, 275]). This behavior was studied for annealing temperatures up to $1600 \mathrm{~K}$, using hyperfine methods [276, 277]. Emission channeling measurements carried out so far cover lattice location studies of implanted ${ }^{33} \mathrm{P}$ [278], ${ }^{8} \mathrm{Li}$ [279], and ${ }^{181} \mathrm{Hf}$ [280]. The PAC measurements, which probe the microstructure of the lattice sites, while confirming these site fractions, however, showed little evidence of defect-free recovering of the lattice even after annealing to temperatures up to $1600 \mathrm{~K}$. Even for those fractions of implanted atoms that give measurable channeling effects, electric field gradients (efg) with appreciable anisotropies were observed.

To the best of our knowledge, no luminescence of lanthanide in bulk diamond is reported in the literature. The only exception are the recent studies of Malashkevich et al. [281], who report on $\mathrm{Eu}^{3+}$-related luminescence in implanted ultradisperse diamond powders. However, oxygen incorporation plays an important role in these studies. But it is important to note that the same strong blue-shift of the ${ }^{5} D_{0} \rightarrow{ }^{7} F_{2}$ transition of $\mathrm{Eu}^{3+}$ is seen as observed for $\mathrm{Eu}^{3+}$ in c-BN studied in this work. Although up to now diamond has fallen short of expectations as a material for light emitters, another application has, for years, been under development: the application of diamond as a particle detector (see e.g. refs. [282, 283, 284, 285]).

\subsubsection{Lattice location of ${ }^{141} \mathrm{Ce}$ and ${ }^{149} \mathrm{Gd}$ in diamond}

To investigate the lattice location of lanthanides implanted into diamond, two isotopes were used for emission channeling investigations for the first time, ${ }^{141} \mathrm{Ce}$ and ${ }^{149} \mathrm{Gd}$. Their corresponding precursors were implanted at ISOLDE, the fluences were $1 \cdot 10^{13} \mathrm{ions} / \mathrm{cm}^{2}$ in the case of ions of mass 139 and $4 \cdot 10^{13}$ ions $/ \mathrm{cm}^{2}$ in the case of ions of mass $149^{2}$ Note that the fluence given in ref. [180] is not correct. The conversion electron energy spectra of the corresponding daughter isotopes ${ }^{141} \mathrm{Pr}$ and ${ }^{149} \mathrm{Eu}$ are shown in figure 3.2.5, together with their decay schemes. In both cases the decay of the implanted precursors is characterized by high Q-factors thus leading to recoil implantation. The samples were therefore stored for some days allowing the main fraction of the ions to decay to ${ }^{141} \mathrm{Ce}$ and ${ }^{149} \mathrm{Gd}$, respectively. After this period, the diamond

\section{2.}




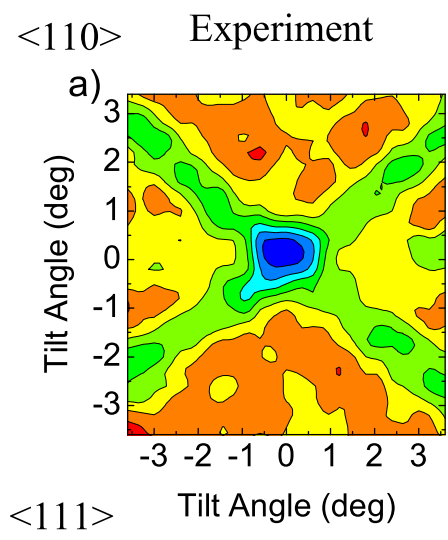

Simulation for near S sites

c)

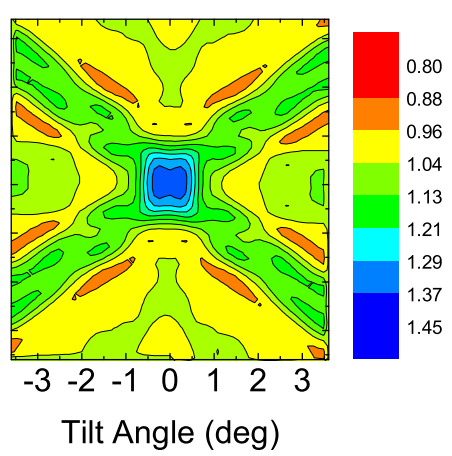

b)

d)
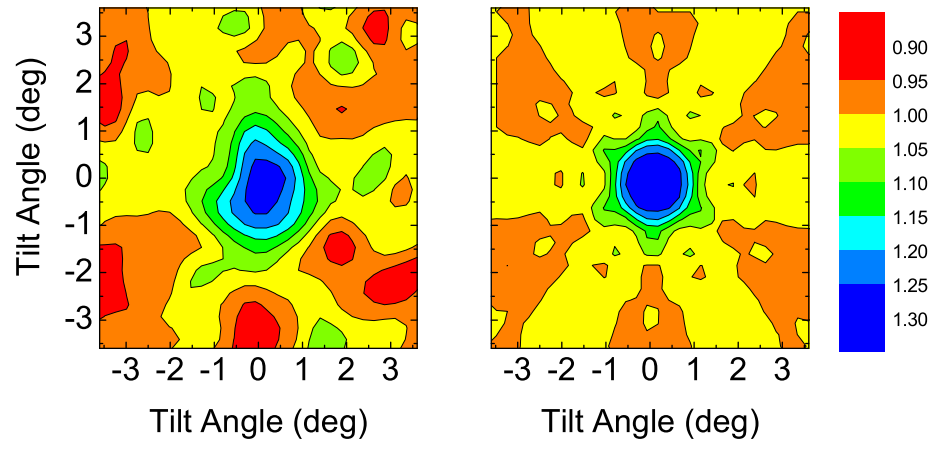

Figure 5.26: (a),(c) Two-dimensional emission channeling raster scan of ${ }^{141} \mathrm{Pr}$ in diamond, recorded using the conversion electrons arising in the decay ${ }^{141} \mathrm{Ce} \rightarrow{ }^{141} \mathrm{Pr}$. The spectra show the normalized electron yield recorded for the $<100>$ and $<110>$ axes, after the sample had been annealed to $1173 \mathrm{~K}$. (b),(d) Simulation corresponding to emitter positions at near substitutional sites. All the spectra were taken from ref. [180].

samples were annealed at moderate temperatures of $1173 \mathrm{~K}$ for 30 minutes in a quartz ampoule, evacuated to approx. $10^{-5}$ mbar. Emission channeling spectra were recorded with the emission channeling setup as described in section 4.2 .

In figure 5.26, the emission channeling spectra of ${ }^{141} \mathrm{Ce}$ in diamond are shown, both for the (a) $<110>$ and (b) $<111>$ axial channeling directions. The axial channeling effects for the $<110>$ and the $<111>$ direction as well as the planar channeling effects corresponding to the $\{111\}$ plane in the $<110>-$ spectrum are clearly visible. In figure 5.26 (c) and (d), the corresponding simulations are shown. The best fits to the experimental spectra were obtained by assuming a near-substitutional lattice site with a displacement of the emitters by $0.51(5) \AA$ towards the $<111>$ direction in the diamond lattice. The fraction of emitter atoms on this site was 
Experiment

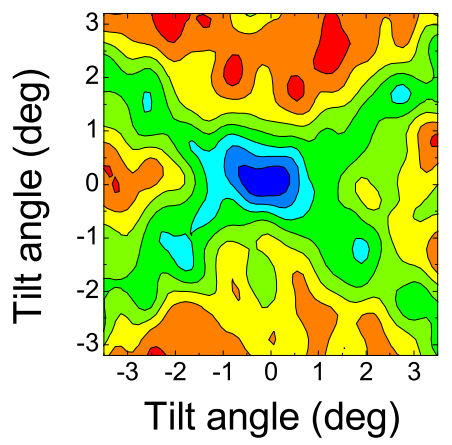

Simulation for near $\mathrm{S}$ site

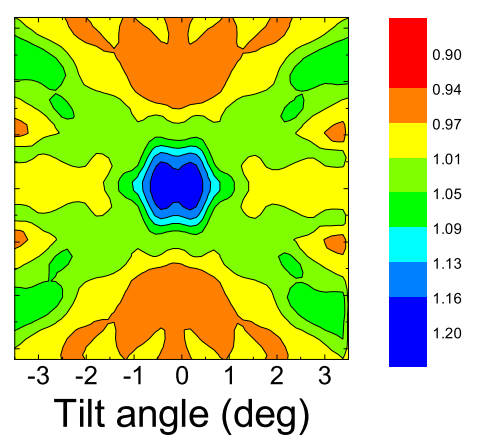

Figure 5.27: Left: two-dimensional emission channeling raster scan of ${ }^{149} \mathrm{Gd}$ in diamond, recorded using the conversion electrons arising from the decay ${ }^{149} \mathrm{Gd} \rightarrow{ }^{149} \mathrm{Eu}$. The spectrum shows the normalized electron yield recorded for the $<110>$ axis, after the sample had been annealed to $1173 \mathrm{~K}$. Right: simulation assuming that the emitter atoms are located on near substitutional carbon sites. The spectra were taken from ref. [180].

determined to be 46(5) \%; the remainder is assumed to be randomly distributed within the diamond lattice. The simple assumption of an ideal substitutional carbon site also resulted in good agreement of experiment and theory, when a mean static displacement of 0.26(2) $\AA$ was considered. Small displacements showing up as a mean static displacement are a general problem of the emission channeling method, as already discussed by Hofsäss et al. [286] and Wahl et al. [213].

In figure 5.27 on the left-hand side, emission channeling spectra of the conversion electrons arising within the decay ${ }^{149} \mathrm{Gd} \rightarrow{ }^{149} \mathrm{Eu}$ are shown. The diamond used in this case had a $\{100\}$ orientation. The emission channeling spectra for the $<100>$ axis were found to be extremely weak, but spectra for the $<110>$ and $<111>$ axes could be recorded successfully. In figure 5.27 on the right-hand side, the corresponding simulations are shown. The emitter atoms were assumed to be located on near-substitutional carbon sites, displaced from an ideal substitutional carbon site by about $0.46(7) \AA$ along the $<111>$ direction in the diamond lattice. The remainder is again assumed to be distributed randomly over the lattice.

In figure 32, the one-dimensional scan around the $<111>$ axis is shown. The best agreement between experimental and simulated spectra was obtained when assuming 48(6) \% of the probe atoms on near-substitutional carbon sites, displaced by $0.5(1) \AA$ from ideal carbon sites along the $<111>$ directions. 


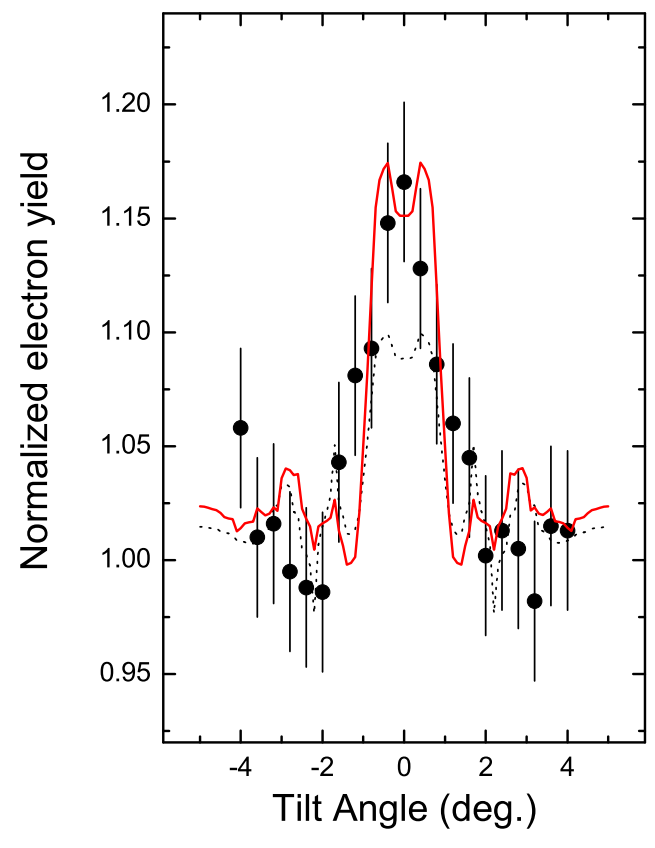

Figure 5.28: One-dimensional emission channeling spectrum of ${ }^{149} \mathrm{Gd}$ in diamond (circles), recorded using the conversion electrons arising in the decay ${ }^{149} \mathrm{Gd} \rightarrow{ }^{149} \mathrm{Eu}$. The spectrum shows the normalized electron yield recorded around the $<111>$ axis, after the sample has been annealed to $1173 \mathrm{~K}$. Simulation (solid line) assuming that the emitter atoms are located on near substitutional carbon sites. The spectra are taken from ref. [180].

\subsubsection{Luminescence properties of thulium and europium in diamond}

Implantation of stable thulium and europium ions (fluence: $1 \cdot 10^{13} \mathrm{ions} / \mathrm{cm}^{2}$ ) was performed at the implantation facility IONAS. After implantation the samples were annealed for 30 minutes under vacuum conditions at a temperature of $1473 \mathrm{~K}$. The CL measurements were performed with the setup described in chapter 4.1. The corresponding CL spectra, recorded at $12 \mathrm{~K}$ under $5 \mathrm{keV}, 0.3 \mathrm{~W} / \mathrm{cm}^{2}$ electron excitation, are shown in figure 5.29. No luminescence due to intra-4f electron transitions of either europium or thulium can be found in the visible range. Instead, the spectra are dominated by defect luminescence, which is described in detail in ref. [287]. Typical transitions such as ${ }^{1} G_{4} \rightarrow{ }^{3} H_{6}$ of $\mathrm{Tm}^{3+}$, which where detectable in AlN, c-BN and $6 \mathrm{H}-\mathrm{SiC}$, seem to be absent or are very weak and masked by the relatively strong defect luminescence. The same holds for the ${ }^{5} D_{0} \rightarrow{ }^{7} F_{2}$ transition of $\mathrm{Eu}^{3+}$. The second derivative spectrum, usually useful to identify weak transitions which are masked by stronger ones, gives no further information. Measurements of the samples used for the emission channeling measurements described above, which contain samarium or praseodymium ions at the end of the corresponding decay chains, showed a similar behavior. The possible reasons for absence of luminescence are manifold in this case, but in the scope of this work they cannot be discussed. A possible explanation may be seen in the microstructure of the lattice sites after ion implantation. As already mentioned, there is little evidence of defect-free recovering of the lattice even after annealing to temperatures up to $1600 \mathrm{~K}$. 

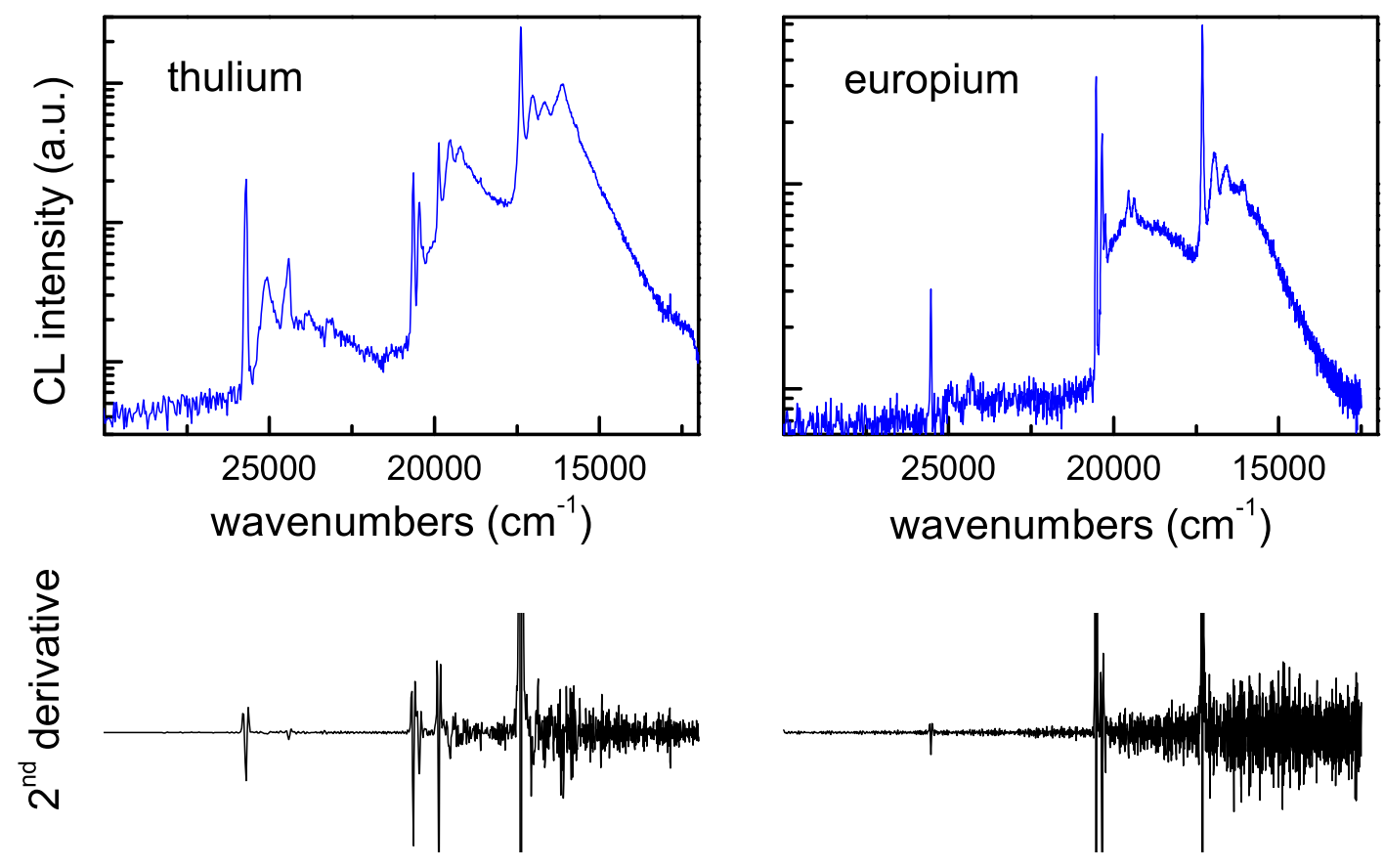

Figure 5.29: $C L$ spectra of europium and thulium implanted diamond, including the second derivative of the spectra. No specific luminescence related to intra-4f electron transitions of $\mathrm{Tm}^{3+}$ and $\mathrm{Eu}^{3+}$ is detected.

Future investigations should be complemented by methods different from those based on luminescence. Questions such as the valence of the ions and the local environment may indeed be better answered by e.g. Mössbauer spectroscopy investigations. Although the implementation of light emitters based on lanthanide implantation will probably never be fruitful, lanthanide implanted diamond is interesting from a more theoretical point of view. The bonding between lanthanide ions and carbon ligands is expected to show pronounced covalent character, making this system interesting for crystal field theory.

\subsubsection{Summary}

Emission channeling measurements have been performed on diamond single crystals implanted with radioactive $\mathrm{Ce}$ and $\mathrm{Gd}$ probe isotopes. After room temperature implantation and annealing at $1200 \mathrm{~K}, 40-50 \%$ of these lanthanide atoms occupy sites displaced from substitutional sites by up to $0.45(10) \AA$ along $<111>$ directions, or with a mean projected displacement 
of $\mathrm{d} \approx 0.26 \AA$. The situation here is different to that observed for lanthanide impurities in $\mathrm{Si}$ [186, 213], where neary $90 \%$ of the implanted probes were observed to take up near-tetrahedralinterstitial sites. 


\section{Thin ta-C and c-BN films doped with Europium during growth}

In the preceding chapters the optical properties of selected lanthanide implanted wide band gap semiconductors were investigated. Obviously, the annealing conditions that are necessary to remove implantation damage and to achieve strong luminescence due to radiative intra- $4 f$ electron transitions of the implanted lanthanides are extreme. A typical example are the hightemperature, high-pressure annealing conditions in the case of c-BN. Although this is not a drawback for basic investigations, an implementation of a light emitter like an LED is almost not applicable under such conditions. An alternative is the doping of these materials during growth. On the other hand it is well-known that years of research in conventional growth techniques such as CVD or MOCVD are necessary to optimize the growth conditions successfully in order to dope such materials with lanthanides. A promising alternative is the growth of thin films by mass selected ion beam deposition (MSIBD), a method used for years in our research group.

In the following the focus will be on thin tetrahedral amorphous carbon as well as cubic boron nitride films grown by MSIBD, because in recent years the main focus has been on investigations of the growth conditions and physical properties of these specimens [229, 223, 222]. The main characteristics of such films are to a large extent determined by the ion energy; the films are either amorphous or nanocrystalline and the thickness of the film is usually in the range of only $50 \mathrm{~nm}$ and therefore much smaller than in the case of CVD or MOCVD grown films

Doping of thin films with lanthanides may follow very different motivations such as the growth of thin films with new optical, electrical or magnetic properties, depending on the matrix, the element used for doping and its concentration [288]. Puzikov et al. observed absorption bands corresponding to $4 \mathrm{f}-5 \mathrm{~d}$ electron transitions of $\mathrm{Ce}$ and $\mathrm{Pr}$ upon incorporation of these lanthanides into hydrogen-free diamond-like carbon [289]. The formation of erbium clusters during deposition onto amorphous carbon was, among others, investigated by Savaloni et al. [290] and $\mathrm{Gu}$ et al. [291]. Recently Liu et al. investigated the doping of thin $\mathrm{B}_{2} \mathrm{~N}_{2} \mathrm{CO}$ films with terbium [54], where successful activation of $\mathrm{Tb}^{3+}$ intra-4f luminescence was achieved. Visible emission from nitrogen-rich turbostratic boron nitride thin films doped with $\mathrm{Eu}, \mathrm{Tb}$, and $\mathrm{Tm}$ was achieved soon after this publication by the same group [51].

The fact that MSIBD-grown c-BN films are nano crystalline makes them interesting systems for doping with lanthanides, as currently there is a great deal of interest in lanthanide doped nano-particles [137, 292, 293, 294, 295, 296, 297]. 
Among the lanthanides $\mathrm{Eu}$ is one of the most interesting elements. $\mathrm{Eu}^{3+}$ is an ideal tool for a basic study of the ion's site using absorption spectroscopy due to low J-values of its ground state and excited $4 \mathrm{f}-$-levels, which are also well separated and therefore easy to assign [106]. These properties were already discussed in chapter 3.1.8. The isotope ${ }^{151} \mathrm{Eu}$ is also accessible to Mössbauer spectroscopy at room temperature [199], which yields additional information on the bonding environment. Although the isomer shift between ${ }^{151} \mathrm{Eu}^{3+}$ and ${ }^{151} \mathrm{Eu}^{2+}$ is very large, the isomer shift between ${ }^{151} \mathrm{Eu}$ ions in different chemical environments but with the same valence is in the range of the quadrupole splitting, which is a major disadvantage of the ${ }^{151} \mathrm{Eu}$ Mössbauer isotope. As already discussed in section 3.5.1, ${ }^{151} \mathrm{Eu}$ is the only useful Mössbauer isotope for thin film investigations.

\subsection{Experimental}

The thin films were grown using the MSIBD facility ADONIS [223] by alternating deposition of ${ }^{12} \mathrm{C}$ and ${ }^{151} \mathrm{Eu}$ ions in the case of ta-C:Eu and ${ }^{11} \mathrm{~B},{ }^{14} \mathrm{~N}$ and ${ }^{151} \mathrm{Eu}$ ions in the case of c-BN:Eu. The ion fraction of Eu offered during growth was set to $0.1 \%$ for both cases.

In the case of the c-BN:Eu films, a buffer layer of 0.15 Coulomb BN (corresponding to a film thickness of approx. $30 \mathrm{~nm}$ ) was deposited onto the silicon substrate before adding ${ }^{151} \mathrm{Eu}$ ions during each deposition cycle. Eu was therefore only added after the formation of textured boron nitride (t-BN) had been finalized, and therefore Eu was incorporated into the top c-BN layer only. All ions were deposited at an energy of $200 \mathrm{eV}$ with the substrate held at $523 \mathrm{~K}$. Approximately $0.1 \mathrm{C}$ (equal to about $20 \mathrm{~nm}$ ) c-BN:Eu was deposited onto the $\mathrm{t}-\mathrm{BN} / \mathrm{c}-\mathrm{BN}$ buffer layer, containing approximately $5 \cdot 10^{14} \mathrm{Eu}$ atoms.

In the case of the ta-C:Eu films a thin buffer layer of ta-C was deposited onto the silicon substrate before adding ${ }^{151} \mathrm{Eu}$ ions during each deposition cycle. The ions were deposited with an energy of $100 \mathrm{eV}$ with the substrate held at room temperature. Films having thicknesses of usually $50 \mathrm{~nm}$, which corresponds to a total amount of approximately $1 \cdot 10^{15} \mathrm{Eu}$ atoms, were deposited.

The X-ray photoelectron spectroscopy (XPS) analysis of all the films was performed in vacuo and $\mathrm{Mg} \mathrm{K}_{\alpha}(\mathrm{h} \nu=1253.6 \mathrm{eV})$ radiation was used to record core level spectra. The typical resolution is $0.9 \mathrm{eV}$ and the spectra, presented without satellite or background correction, were measured with constant pass energy. The indicated energies refer to the Fermi level and the $\mathrm{Au} 4 \mathrm{f}_{7 / 2}$ line (positioned at $84.0 \mathrm{eV}$ ) of a clean gold sample. None of the films exhibited any charging during the photoelectron spectroscopy analysis. 
Two different setups were used for conversion electron Mössbauer spectroscopy (CEMS). In the first setup, a $50 \mathrm{mCi}^{151} \mathrm{SmF}_{3}$ source and a parallel plate avalanche counter [218], using pure acetone as detector gas, were used. In the second setup a $350 \mathrm{mCi}^{151} \mathrm{SmF}_{3}$ source and a gas flow proportional counter using $\mathrm{He} / \mathrm{CH}_{4}$ as detector gas were used. The latter setup is described in chapter 4.3.2. For velocity calibration a ${ }^{57} \mathrm{Co}$ source and an $\alpha-\mathrm{Fe}$ absorber were used. The velocities are finally given for a commercially available single line $\mathrm{Eu}_{2} \mathrm{O}_{3}$ powder absorber, measured with the same CEMS detectors as the thin films. Additionally radioactive ${ }^{151} \mathrm{Gd}$ ions were implanted at ISOLDE at an energy of $60 \mathrm{keV}$ into thin ta-C and c-BN films of approximately $100 \mathrm{~nm}$ in thickness. Transmission Mössbauer spectroscopy was performed at the decay ${ }^{151} \mathrm{Gd} \rightarrow{ }^{151} \mathrm{Eu}$ using a conventional $\mathrm{Eu}_{2} \mathrm{O}_{2}$ powder absorber. The corresponding $\mathrm{X}$-ray spectrum of ${ }^{151} \mathrm{Eu}$ is shown in Figure 3.11

Cathodoluminesence (CL) spectroscopy was performed with the setup described in chapter 4.1

\subsection{Results}

Figure 6.1 shows the in vacuo photoelectron spectra for both the c-BN:Eu and the ta-C:Eu thin film. In the case of the Eu co-deposited ta-C film, the concentration of Eu which is obtained from the highly surface sensitive XPS is $\sim 6 \%$ for Eu and $\sim 9.5 \%$ for oxygen. It is therefore

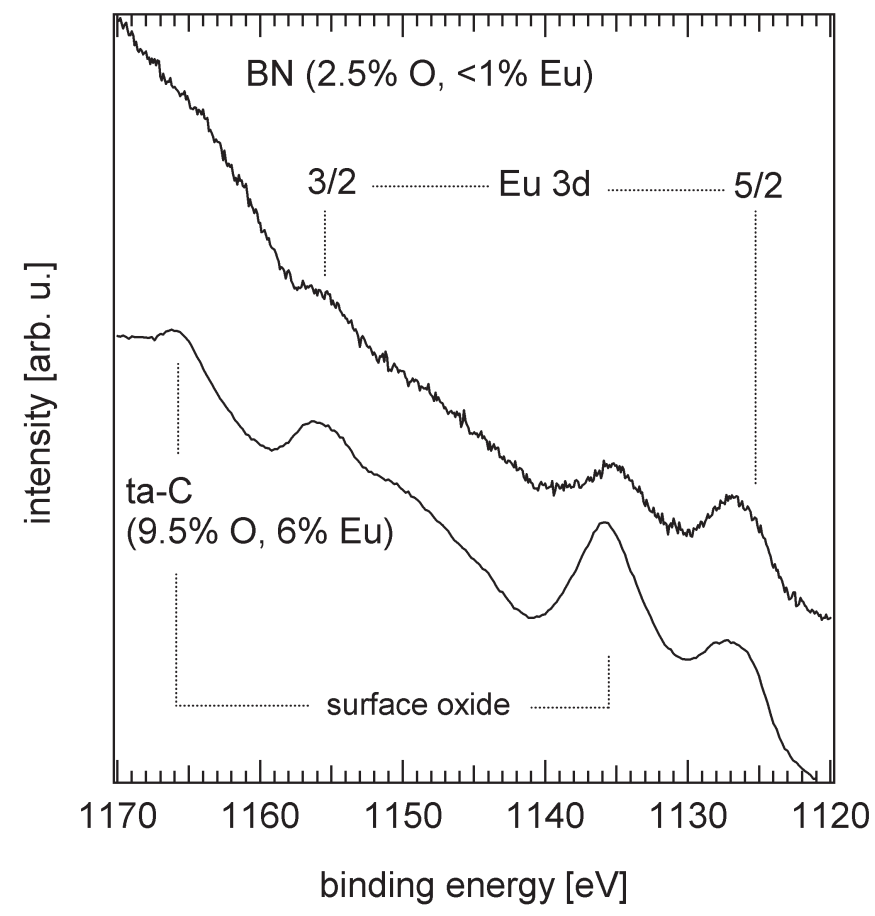

Figure 6.1: XPS spectra of the Eu $3 \mathrm{~d}$ core level for the ta-C:Eu and c$\mathrm{BN}: \mathrm{Eu}$ thin films [53]. The surface concentrations of europium and oxygen are indicated in the figure. 


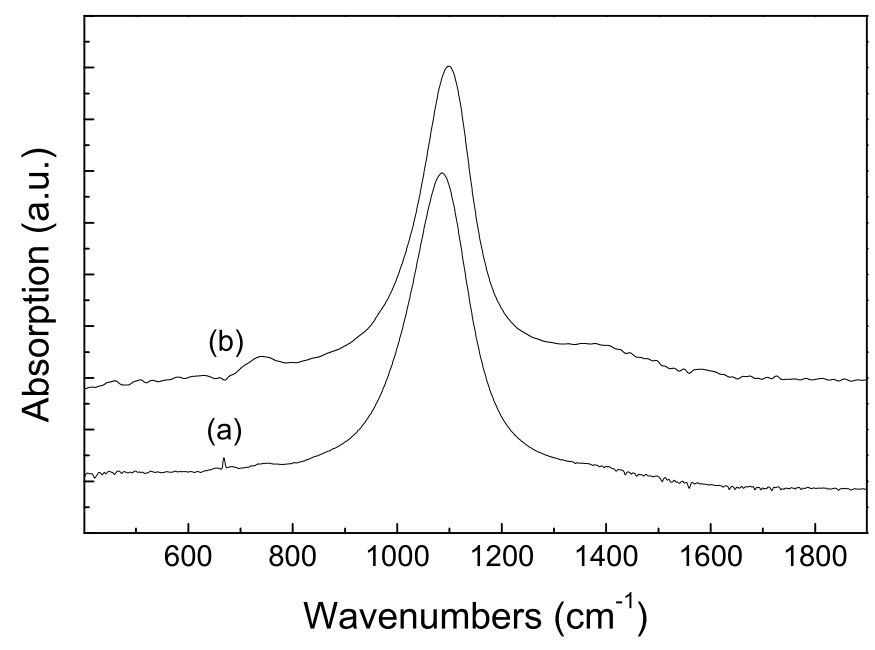

Figure 6.2: (a) FTIR spectra of (a) undoped and (b) Eu-codoped c$\mathrm{BN}$ thin films grown under the same conditions [53].

likely that almost all deposited europium atoms are located near or at the surface layer and are partially oxidized. A similar segregation behavior was recently shown upon doping of MSIBD grown ta-C films with silver during growth [298]. In the case of the BN:Eu films an upper limit of $1 \%$ of europium and about $2.5 \%$ of oxygen was detected and again Eu was partially oxidized. The concentration of europium near the surface is, however, much smaller compared to that of the ta-C:Eu film and a surface segregation of Eu to a large extent appears unlikely.

To confirm the existence of $\mathrm{c}-\mathrm{BN}$ in the case of the BN:Eu films, ex situ Fourier transform infrared spectroscopy was applied, showing mainly characteristic reststrahlen-absorption of c$\mathrm{BN}$ at ca. $1080 \mathrm{~cm}^{-1}$. Fig. 6.2 shows FTIR spectra of Eu-doped and undoped c-BN thin films grown under the same conditions. From the spectra it is evident that the c-BN structure is preserved during addition of Eu. CEMS measurements of the c-BN:Eu thin films (Fig. 6.3 (a)) indicate Eu ions in the charge state $3^{+}$with respect to the observed isomer shift. A line splitting is observed which may be fitted with several lines whose line widths correspond to the natural line width of the ${ }^{151} \mathrm{Eu}$ Mössbauer transition, which is $\Gamma \approx 1.3 \mathrm{~mm} / \mathrm{s}$ (FWHM). A possible fitting of the measured spectrum with several Lorentzian lines is indicated in Fig. 6.3 (a), although fitting of the spectrum without knowledge of the electric field gradient (EFG) is difficult as each EFG leads to 8 (12) hyperfine transitions in the case of the asymmetry parameter $\eta=0(\eta \neq 0)$. The evaluation is even more complicated by the fact that MSIBD grown c-BN films consist of partially oriented nano-crystals whose $\{111\}$-axes are aligned parallel to the incident ion beam. Finally the top oxidized Eu layer seen with XPS has to be taken into account as well. One should also keep in mind the two possible sites of the europium ions in the cBN lattice, as discussed in section 5.2.2. However, the XPS and CEMS measurements cannot 
a)

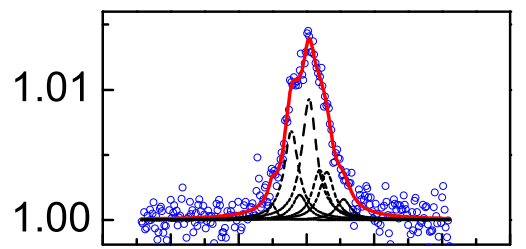

b)

?.?

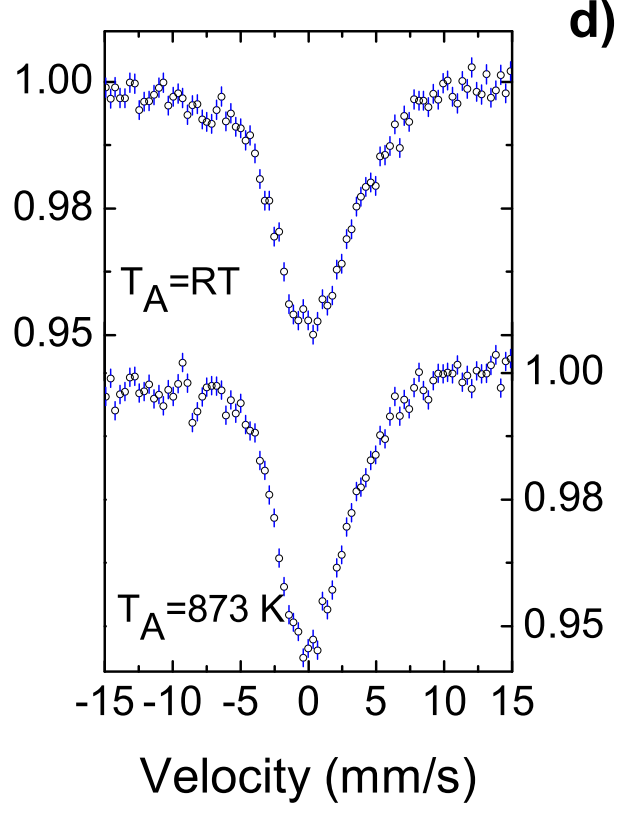

c)

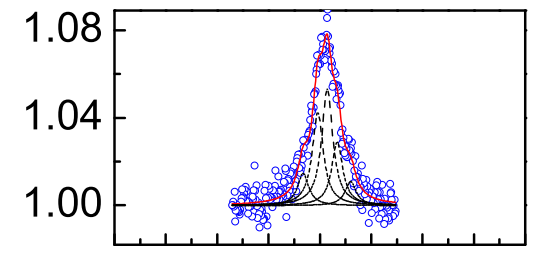

d)

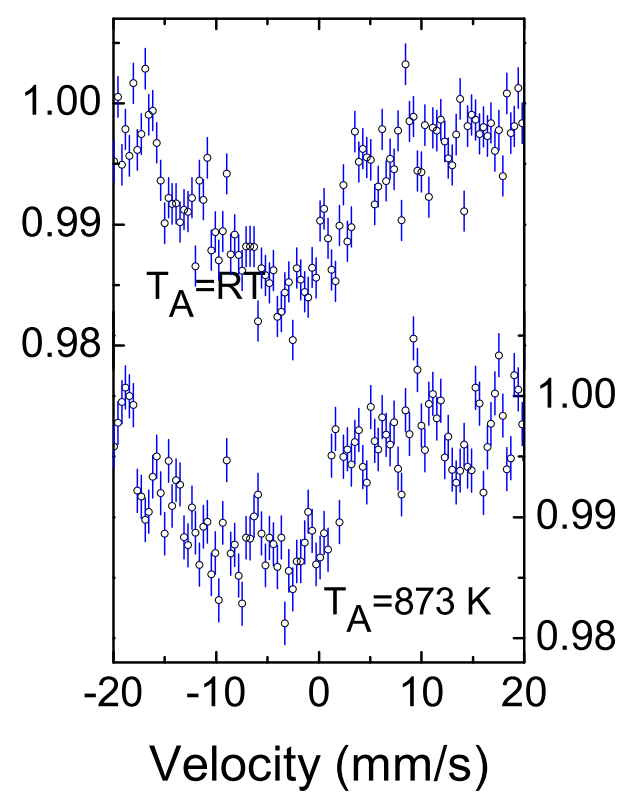

Figure 6.3: (a) ${ }^{151} \mathrm{Eu}$ CEMS spectrum of a ${ }^{151} \mathrm{Eu}-$ codeposited c-BN thin film. (b) ${ }^{151} \mathrm{Eu}$ TMS spectrum of a ${ }^{151} \mathrm{Gd}$-implanted c-BN thin film. (c) ${ }^{151}$ Eu CEMS spectrum of a ${ }^{151} \mathrm{Eu}$ co-deposited ta-C thin film. (d) ${ }^{151} \mathrm{Eu}$ TMS spectrum of a ${ }^{151} \mathrm{Gd}$-implanted ta-C thin film. The velocity is given with respect to a $\mathrm{Eu}_{2} \mathrm{O}_{3}$ absorber.

be compared directly, because firstly CEMS probes, in contrast to XPS, the bulk of the film, and secondly the surface layer probed with XPS is likely to further oxidize ex vacuo. Figure 6.3 (b) shows a ${ }^{151} \mathrm{Eu}$ Mössbauer spectrum of a thin c-BN film implanted with corresponding radioactive precursors which decay to ${ }^{151} \mathrm{Gd}$. The latter one has a half-life of $128 \mathrm{~d}$ and decays to ${ }^{151} \mathrm{Eu}$ which leads to the emission of the characteristic Mössbauer radiation. The energy spectrum is shown in figure 3.11 . Spectrum 6.3 (b), indicating no isomer shift, suggests that the valence of the ${ }^{151} \mathrm{Eu}$ ions in the $\mathrm{c}-\mathrm{BN}$ host is $3+$ like in the case of the co-deposited films. There is a structure in the spectra which may again be interpreted as either several isomer shifts or as due to a crystal field splitting. Annealing the implanted films to a temperature of $873 \mathrm{~K}$ 


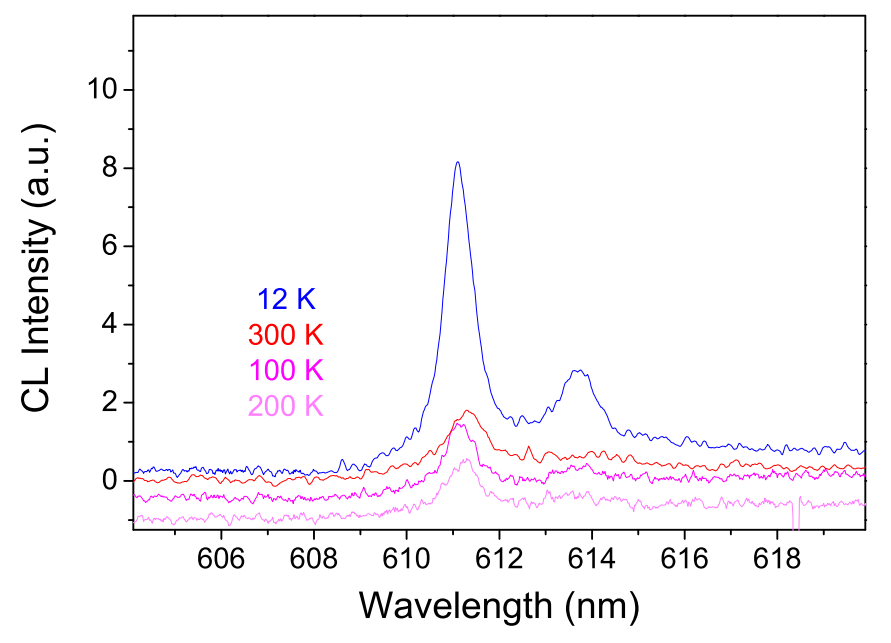

Figure 6.4: $\mathrm{CL}$ spectra of the ${ }^{5} D_{0} \rightarrow{ }^{7} F_{2}$ intra-4f electron transition of $\mathrm{Eu}^{3+}$ doped into c-BN during growth, measured at different temperatures under $5 \mathrm{keV}$, $0.3 \mathrm{~W} / \mathrm{cm}^{2}$ electron excitation.

for 10 minutes under vacuum conditions did not change the situation significantly.

Cathodoluminescence measurements (Fig. 6.4) indicate that at least a fraction of Eu ions occupies well-defined sites in the $\mathrm{c}-\mathrm{BN}$ lattice, which we assume to be near substitutional boron sites. The CL spectrum, recorded at $12 \mathrm{~K}\left(5 \mathrm{keV}, 0.3 \mathrm{~W} / \mathrm{cm}^{2}\right.$ electron excitation), shows a weak luminescence, but with a clear splitting of the ${ }^{5} \mathrm{D}_{0} \rightarrow{ }^{7} \mathrm{~F}_{2}$ intra-4f electron transition of $\mathrm{Eu}^{3+}$ indicating a well defined environment of the Eu ions. This luminescence is, although still visible, quenched at room temperature. Lifetime measurements with pulsed CL show a very fast decay of the ${ }^{5} \mathrm{D}_{0}$ level which is below the current time resolution of our setup $(\sim 1 \mu \mathrm{s})$. These results can directly be compared to the europium implanted c-BN specimens, which were discussed in section 5.2.2. Obviously, the large crystal field splitting which was detected in the case of the $\mathrm{Eu}-\mathrm{implanted} \mathrm{c}-\mathrm{BN}$ poly crystals is absent. On the other hand, the energetic positions of the ${ }^{5} D_{0} \rightarrow{ }^{7} F_{2}$ transition shown in Figure 6.4 coincide well with some luminescence lines related to $\mathrm{Eu}^{3+}$ ions in the poly crystal sample. Possible explanations for the overall weak Eu ${ }^{3+}$-related luminescence in the $\mathrm{c}-\mathrm{BN}$ thin film are provided by the probably low solubility of europium in $\mathrm{c}-\mathrm{BN}$ and carrier relaxation at $\mathrm{c}-\mathrm{BN}$ grain boundaries and the $\mathrm{Si} / \mathrm{t}-\mathrm{BN} / \mathrm{c}-\mathrm{BN}$ heterojunction. The latter facts might be responsible for efficient non-radiative backtransfer resulting in the observed fast lifetime of the ${ }^{5} D_{0}$. An important observation is the remarkable shift of the ${ }^{5} D_{0} \rightarrow{ }^{7} F_{2}$ transition to longer wavelengths, which was also observed by Malashkevich et al. [281] for $\mathrm{Eu}^{3+}$-implanted ultradisperse diamond powders. An example of the ${ }^{5} D_{0} \rightarrow{ }^{7} F_{2}$ transition found at energies predicted by the mean free ion parameters, are the spectra reported for $\mathrm{Eu}^{3+}$ in $\mathrm{AlN}$ in this work.

Considering the ex situ CEMS spectra of the codeposited ta-C:Eu (shown in Fig. 6.3(b)) it can 
be seen that Eu exists in the charge state $3^{+}$, although there is a structure within the spectrum indicating more than one local environment of the Eu ions. It is suggested that the structure does not originate from a single EFG leading to a quadrupole splitting, but more likely the Eu atoms bound to hydrogen, oxygen, hydroxy and other groups [299]. On the other hand, as regards the observation of erbium cluster formation on amorphous carbon mentioned before [290, 291], this alternative has to be considered as well. However, in the scope of the methods used, there is no proof of this. Although in situ and ex situ measurements are comparable as regards the observation that Eu is located close to or at the surface and both the XPS and CEMS method should therefore probe the same layer of approx. $1 \mathrm{~nm}$ in thickness, it is likely that the Eu layer is modified after the XPS measurements due to the interaction with air. However one may not fully exclude the incorporation of Eu atoms into ta-C to a small extent. Figure 6.3 (d) shows the corresponding TMS spectrum of the ${ }^{151} \mathrm{Gd}$-implanted thin ta-C films, prepared and measured under the same conditions as in the case of the implanted c-BN films discussed above. It can be seen that in this case the situation between co-deposited and implanted films is very different. The TMS spectrum shows only a moderate absorption due to a possibly low Debye-Waller-factor and very broad absorption bands. This points to an amorphous local environment of the thin films. Moreover, an absorption band at around $10-15 \mathrm{~mm} / \mathrm{s}$ is visible, an indication of europium atoms in the charge state $2+$. Upon annealing the sample at $873 \mathrm{~K}$ for 10 minutes under vacuum conditions the broad band at negative velocities even increases. A surface segregation or outdiffusion is, however, not observed.

\subsection{Summary}

The doping of MSIBD grown ta-C and $\mathrm{c}-\mathrm{BN}$ films with europium was investigated. The films were doped with Eu during growth by co-deposition. The application of different methods, such as photoelectron, cathodoluminescence or conversion electron Mössbauer spectroscopy, for the investigation of such films was shown. Successful incorporation of europium in the charge state $3+$ and activation of $\mathrm{Eu}^{3+}$ intra-4f luminescence was achieved for co-deposited c$\mathrm{BN}$ :Eu films. In the case of the ta-C:Eu film, an accumulation of $\mathrm{Eu}$ at the surface was observed and an incorporation of Eu into the ta-C matrix seemed to be difficult. For both ta-C and c-BN films the host matrix was preserved. 


\section{Crystal field analysis for $\mathbf{T m}^{3+}\left(4 f^{12}\right)$ and $\mathbf{G d}^{3+}\left(4 f^{7}\right)$ in AlN}

In the last chapters, the investigation of optical properties of $\mathrm{Tm}^{3+}, \mathrm{Eu}^{3+}$ and $\mathrm{Gd}^{3+}$ doped wide band gap semiconductors $\mathrm{AlN}, \mathrm{c}-\mathrm{BN}, 6 \mathrm{H}-\mathrm{SiC}$, and diamond by means of cathodoluminescence from the near infrared to the ultra violet region of the electromagnetic spectrum was discussed. Successful activation of luminescence related to these lanthanides was achieved for $\mathrm{Tm}^{3+}$ in $\mathrm{AlN}, \mathrm{c}-\mathrm{BN}$ as well as $6 \mathrm{H}-\mathrm{SiC}$, for $\mathrm{Eu}^{3+}$ in $\mathrm{AlN}$ and $\mathrm{c}-\mathrm{BN}$ and for $\mathrm{Gd}^{3+}$ in AlN. Regarding possible crystal field analyses, only a few systems are suitable. It was already pointed out that $\mathrm{Eu}^{3+}$ is not suitable for a crystal field analysis in luminescence investigations because of the low J-values of the final transitions which appear in the optical region of interest. For $\mathrm{Tm}^{3+}$ the number of transitions detected in the case of $6 \mathrm{H}-\mathrm{SiC}$ is insufficient, and the fact that more than one possible is expected for the ions in the $6 \mathrm{H}-\mathrm{SiC}$ host requires different approaches, such as time-resolved measurements, to distinguish between these sites via cathodoluminescence spectroscopy. Although $\mathrm{Tm}^{3+}$-related luminescence in the $\mathrm{c}-\mathrm{BN}$ host shows promising properties, it is evident that the ions are located on sites of low symmetry, which very likely belong to a certain defect configuration. In contrast to these drawbacks, the AlN host does meet all the requirements for a successful crystal field analysis. The number of transitions between individual Stark levels is large in the case of $\mathrm{Tm}^{3+}$ and there is no evidence of many sites of the implanted ions. It therefore suggested itself to perform a crystal field analysis for $\mathrm{Tm}^{3+}$ in $\mathrm{AlN}$. Additionally it was noticed that the optical transitions related to $\mathrm{Gd}^{3+}$ in AlN are suitable for a crystal field analysis as well. Both systems will be discussed in this chapter.

In a first approach, the software provided by Mike Reid, which was modified as described in chapter 3.1.7, was used for the initial calculations. This allowed to identify many of the transitions between multiplet manifolds, based on roughly calculated branching ratios as well as crystal field calculations using the mean free ion parameters listed in table A.1. The major problem that had to be overcome was the fitting of the crystal field parameters to transitions between the individual Stark levels. Taking crystal field parameters and fitting them towards these transitions may result in with parameters very different from the ones found in the literature. The reason is obvious: a free variation of the crystal field parameters may lead to solutions that are physically meaningless. It is therefore extremely important to have a reliable set of

crystal field parameters which have to be varied only in small ranges, or the variation has to be constrained to physically reasonable sets of parameters.

Most of the assignments of luminescence lines to transitions between certain Stark levels were 
CHAPTER 7. CRYSTAL FIELD ANALYSIS FOR TM ${ }^{3+}\left(4 F^{12}\right)$ AND GD ${ }^{3+}\left(4 F^{7}\right)$ IN ALN

kindly provided by Dr. John B. Gruber, University of San José, California. At present, these assignments as well as the calculated crystal field parameters have to be considered as preliminary and may be subject to minor changes in a final publication.

In section 3.1 .3 an expression for the potential at the $4 f$-electron was given. Following the notation of Gruber et al. [160, 300, 250, 31, 33, 38], the complete crystal field Hamiltonian of the $4 f$ electron system is written as:

$$
H_{C F}=\sum_{n \text { even }} \sum_{m=-n}^{n} B_{n m}^{*} \sum_{i=1}^{N} C_{n m}\left(r_{i}\right),
$$

with the normalized one-electron tensor operators $C_{n m}\left(r_{i}\right)$ defined by:

$$
C_{n m}\left(r_{i}\right)=\sqrt{\frac{4 \pi}{2 n+1}} Y_{n m}\left(\theta_{i}, \phi_{i}\right)
$$

and

$$
C_{n,-m}\left(r_{i}\right)=(-1)^{m} C_{n m}^{*}\left(r_{i}\right)
$$

where $\mathrm{n}=2,4,6$ and $\mathrm{m}=0, \pm 3$, and \pm 6 , for $|m| \leq n$. The sum on $i$ runs over all $N 4 f$ electrons, $i=14(2)$ for $\mathrm{Tm}^{3+}\left(4 f^{12}\right)$ and $i=7$ for $\mathrm{Gd}^{3+}\left(4 f^{7}\right)$. This notation is almost the same as the one given in chapter 3.1.3, except that $k$ and $q$ are replaced by $n$ and $m$.

Rather than performing $a b$ initio lattice sum calculations, exisiting crystal field parameter sets for $\mathrm{Sm}^{3+}$ and $\mathrm{Tb}^{3+}$ were used. These existing parameter sets cover both $\mathrm{C}_{3 v}$ and $\mathrm{D}_{2}$ symmetry. The reported crystal field parameters for $\mathrm{Sm}^{3+}$ in $\mathrm{GaN}\left(\mathrm{C}_{3 v}\right)$ are [33]: $B_{20}=-48.9, B_{40}=$ $-860.7, B_{43}=-321.9, B_{60}=692.7, B_{63}=-265.7, B_{66}=261.8$, and for $\mathrm{Sm}^{3+}$ in $\mathrm{GaN}$ $\left(\mathrm{D}_{2}\right)$ [38]: $B_{20}=505, B_{22}=162, B_{40}=-424, B_{42}=-1092, B_{44}=-1147, B_{60}=-1053$, $B_{62}=-430, B_{64}=146, B_{66}=-298$. All $B_{n m}$ are in units of wavenumbers. 


\subsection{Experimental CL Stark level spectra of $\mathbf{T m}^{3+}$ in AIN}

In section 5.1 .4 the properties of $\mathrm{Tm}^{3+}$-implanted AlN were presented. These samples were either implanted at ISOLDE at an energy of $60 \mathrm{keV}$ or at IONAS at an energy of $100 \mathrm{keV}$ and fluences of $1 \cdot 10^{13}$ ions $/ \mathrm{cm}^{2}$. As already mentioned, the ISOLDE samples contained minor contaminations of lanthanide oxides such as GdO or EuO. Therefore the samples used for crystal field investigations were the ones implanted at IONAS. These samples were, subsequent to implantation, annealed under vacuum conditions in a tube furnace at $1373 \mathrm{~K}$ for 30 minutes.

In Figures 7.1 and 7.2 the complete spectra of all detectable transitions between individual Stark levels in the wavelength range of $200-850 \mathrm{~nm}$ are shown. These spectra were recorded at $12 \mathrm{~K}$ under standard excitation conditions $\left(5 \mathrm{keV}, 0.3 \mathrm{~W} / \mathrm{cm}^{2}\right)$ using the setup described in chapter 4.1. The luminescent transitions are presented in either logarithmic or linear scales; intensities were not assigned as the spectra were recorded using different resolutions of the setup. The survey scan shown in Figure 5.8 gives an idea of these intensities. The position of the individual lines was extracted either by direct fitting with Lorentzian or Gaussian distributions or by forming the second derivative of the spectra. This idea is based on the second derivative units sometimes installed at fluorescence spectrometers, an example can be found in the work of Lakshman and Jayasankar [161]. Whether other authors also use the computational method used in this work is not clear. Sharp luminescence lines appear in the second derivate spectra in the form of local minima with a minimum value of less than zero. This approach was found to be very useful, but is only applicable in case the luminescence is relatively strong - otherwise the magnitude of the 2nd derivative is too close to the background.

Table 7.1 shows the complete list of transitions extracted from the experimental spectra. The luminescence lines are consecutively numbered and correspond to those given in Figures 7.1 and 7.2. The total number of individual transitions exceeds 200 , mostly because the $\mathrm{Tm}^{3+}$ energy levels are sufficiently spaced to prevent non-radiative deexcitation. The assignment of these lines to transitions between individual Stark levels or even groups of lines to transitions between multiplet manifolds is, however, not straightforward, because many transitions between multiplet manifolds overlap for $\mathrm{Tm}^{3+}$. It is often mentioned in the literature that the energetic positions of free ion energy levels are almost unaffected by the crystal field. Although this is true, the small influence may lead to some confusion as variations of free ion energy levels can still be in the range of a few hundred wave numbers. A typical example of very closely spaced transitions between multiplet manifolds in the case of $\mathrm{Tm}^{3+}$ are the transitions ${ }^{1} I_{6} \rightarrow{ }^{3} H_{4},{ }^{1} D_{2} \rightarrow{ }^{3} F_{4}$ and ${ }^{1} G_{4} \rightarrow{ }^{3} H_{6}$ in the range of $455-490 \mathrm{~nm}$. 
CHAPTER 7. CRYSTAL FIELD ANALYSIS FOR TM ${ }^{3+}\left(4 F^{12}\right)$ AND GD ${ }^{3+}\left(4 F^{7}\right)$ IN ALN
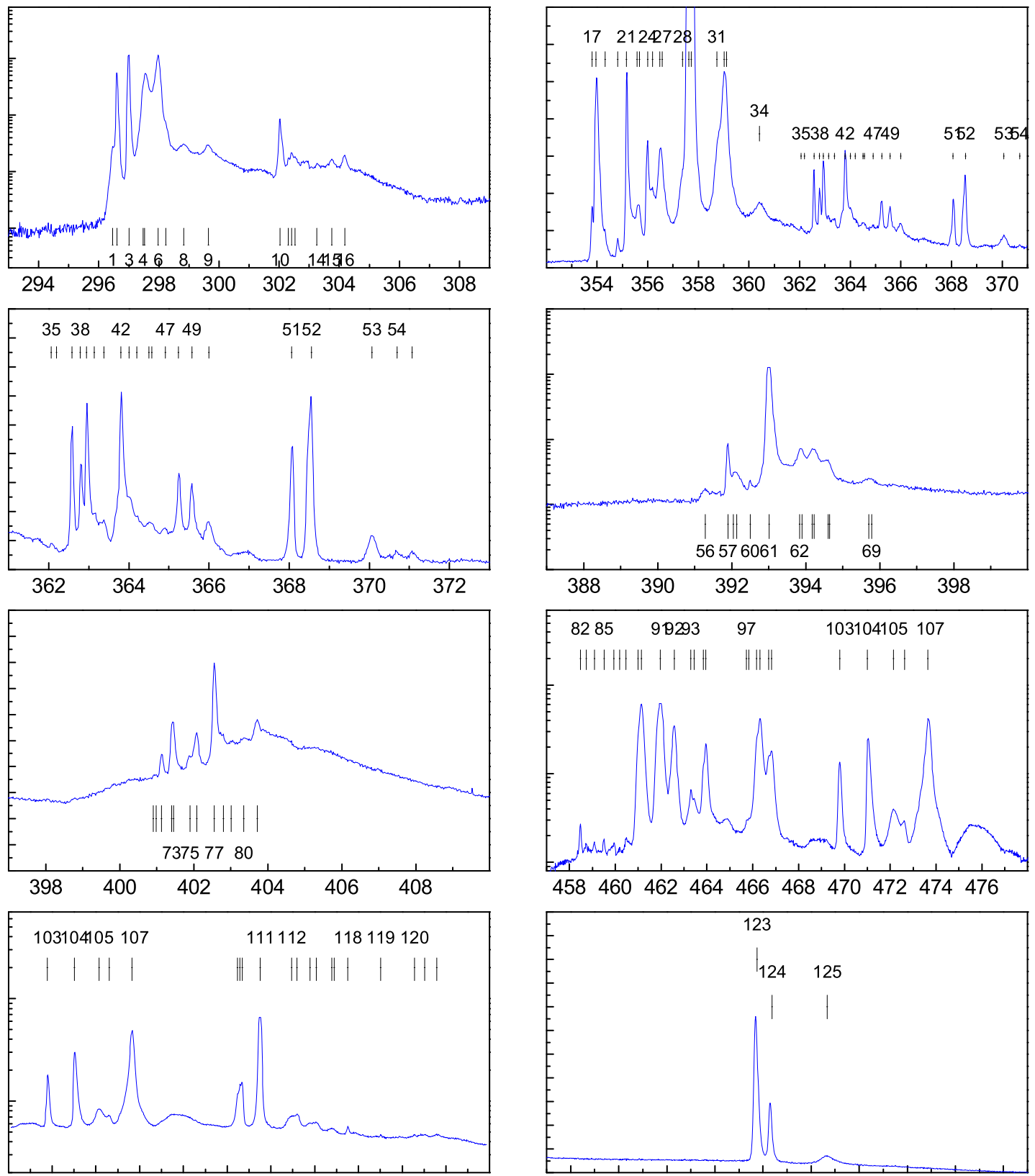

470472474476478480482484486488

Wavelength $(\mathrm{nm})$

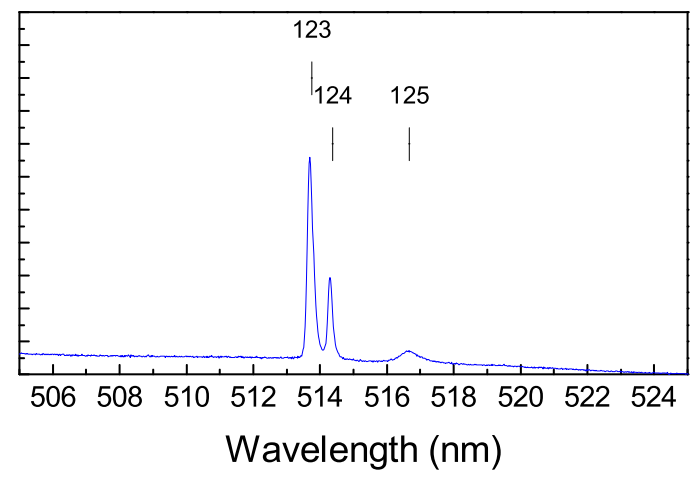

Figure 7.1: High resolution CL spectra of $\mathrm{Tm}^{3+}$ implanted AlN, part I. All the spectra were recorded at $12 \mathrm{~K}$. Individual transitions are numbered consecutively (compare table 7.1). 


\subsection{EXPERIMENTAL CL STARK LEVEL SPECTRA OF TM ${ }^{3+}$ IN ALN}
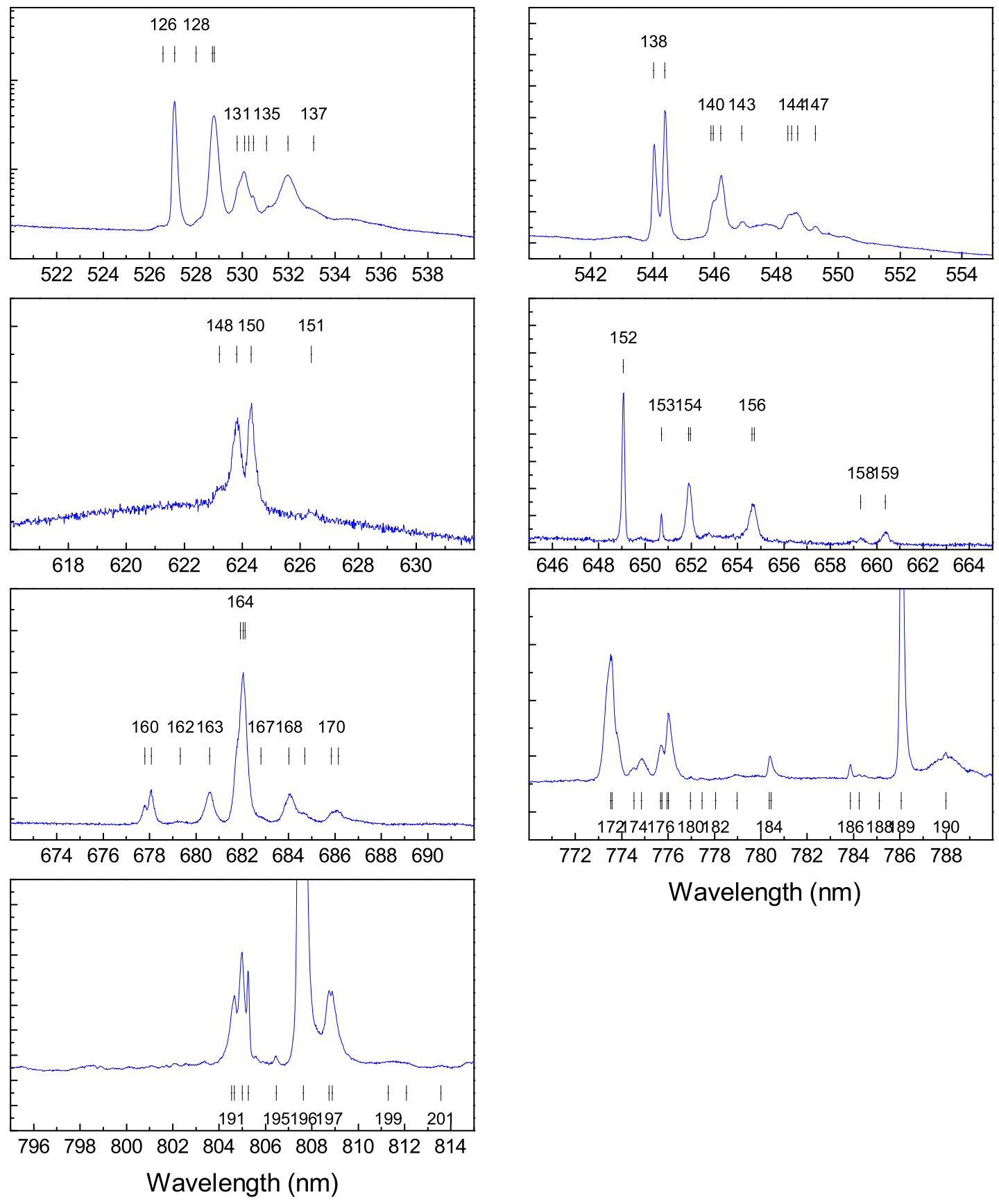

Figure 7.2: High resolution CL spectra of $\mathrm{Tm}^{3+}$ implanted AlN, part II. All the spectra were recorded at $12 \mathrm{~K}$. Individual transitions are numbered consecutively (compare table 7.1 . 
CHAPTER 7. CRYSTAL FIELD ANALYSIS FOR TM ${ }^{3+}\left(4 F^{12}\right)$ AND GD ${ }^{3+}\left(4 F^{7}\right)$ IN ALN

\begin{tabular}{|c|c|c|c|c|c|c|c|c|}
\hline No. $^{\mathrm{a}}$ & $\begin{array}{c}\lambda^{\mathrm{b}} \\
(\mathrm{nm})\end{array}$ & $\begin{array}{c}\mathrm{E}^{\mathrm{C}}{ }_{o b s} \\
\left(\mathrm{~cm}^{-1}\right)\end{array}$ & No. $^{\mathrm{a}}$ & $\begin{array}{c}\lambda^{\mathrm{b}} \\
(\mathrm{nm})\end{array}$ & $\begin{array}{c}\mathrm{E}^{\mathrm{c}}{ }_{o b s} \\
\left(\mathrm{~cm}^{-1}\right)\end{array}$ & $\overline{\text { No. }^{a}}$ & $\begin{array}{c}\lambda^{\mathrm{b}} \\
(\mathrm{nm})\end{array}$ & $\begin{array}{c}\mathrm{E}^{\mathrm{C}}{ }^{\mathrm{C} s} \\
\left(\mathrm{~cm}^{-1}\right)\end{array}$ \\
\hline 1 & 296.5 & 33722 & 46 & 364.6 & 27422 & 91 & 462.0 & 21641 \\
\hline 2 & 296.6 & 33706 & 47 & 364.9 & 27396 & 92 & 462.6 & 21612 \\
\hline 3 & 297.0 & 33660 & 48 & 365.2 & 27372 & 93 & 463.3 & 21579 \\
\hline 4 & 297.5 & 33607 & 49 & 365.6 & 27347 & 94 & 463.4 & 21572 \\
\hline 5 & 297.5 & 33601 & 50 & 366.0 & 27315 & 95 & 463.8 & 21553 \\
\hline 6 & 298.0 & 33551 & 51 & 368.1 & 27162 & 96 & 463.9 & 21548 \\
\hline 7 & 298.2 & 33522 & 52 & 368.6 & 27125 & 97 & 465.7 & 21466 \\
\hline 8 & 298.8 & 33454 & 53 & 370.1 & 27014 & 98 & 465.8 & 21462 \\
\hline 9 & 299.6 & 33363 & 54 & 370.7 & 26969 & 99 & 466.2 & 21446 \\
\hline 10 & 302.0 & 33100 & 55 & 371.1 & 26942 & 100 & 466.3 & 21439 \\
\hline 11 & 302.3 & 33069 & 56 & 391.3 & 25550 & 101 & 466.7 & 21422 \\
\hline 12 & 302.4 & 33057 & 57 & 391.9 & 25510 & 102 & 466.8 & 21416 \\
\hline 13 & 302.5 & 33045 & 58 & 392.1 & 25500 & 103 & 469.8 & 21280 \\
\hline 14 & 303.3 & 32966 & 59 & 392.1 & 25494 & 104 & 471.0 & 21225 \\
\hline 15 & 303.8 & 32911 & 60 & 392.5 & 25471 & 105 & 472.1 & 21174 \\
\hline 16 & 304.2 & 32866 & 61 & 393.0 & 25438 & 106 & 472.6 & 21153 \\
\hline 17 & 353.8 & 28257 & 62 & 393.8 & 25385 & 107 & 473.6 & 21107 \\
\hline 18 & 353.9 & 28245 & 63 & 393.9 & 25380 & 108 & 478.5 & 20895 \\
\hline 19 & 354.3 & 28215 & 64 & 394.2 & 25362 & 109 & 478.6 & 20889 \\
\hline 20 & 354.8 & 28176 & 65 & 394.2 & 25359 & 110 & 478.7 & 20885 \\
\hline 21 & 355.2 & 28149 & 66 & 394.6 & 25335 & 111 & 479.5 & 20849 \\
\hline 22 & 355.6 & 28114 & 67 & 394.6 & 25332 & 112 & 480.9 & 20787 \\
\hline 23 & 355.7 & 28108 & 68 & 395.7 & 25265 & 113 & 481.2 & 20776 \\
\hline 24 & 356.0 & 28082 & 69 & 395.8 & 25260 & 114 & 481.8 & 20751 \\
\hline 25 & 356.2 & 28067 & 70 & 400.9 & 24936 & 115 & 482.1 & 20738 \\
\hline 26 & 356.5 & 28044 & 71 & 401.0 & 24932 & 116 & 482.8 & 20708 \\
\hline 27 & 356.6 & 28036 & 72 & 401.1 & 24923 & 117 & 482.9 & 20703 \\
\hline 28 & 357.4 & 27974 & 73 & 401.4 & 24906 & 118 & 483.5 & 20676 \\
\hline 29 & 357.6 & 27954 & 74 & 401.4 & 24903 & 119 & 485.0 & 20613 \\
\hline 30 & 357.7 & 27947 & 75 & 401.9 & 24875 & 120 & 486.6 & 20547 \\
\hline 31 & 358.7 & 27868 & 76 & 402.1 & 24863 & 121 & 487.0 & 20527 \\
\hline 32 & 359.0 & 27845 & 77 & 402.6 & 24834 & 122 & 487.6 & 20504 \\
\hline 33 & 359.1 & 27838 & 78 & 402.8 & 24819 & 123 & 513.8 & 19459 \\
\hline 34 & 360.4 & 27739 & 79 & 403.0 & 24806 & 124 & 514.4 & 19436 \\
\hline 35 & 362.1 & 27612 & 80 & 403.4 & 24786 & 125 & 516.7 & 19350 \\
\hline 36 & 362.2 & 27601 & 81 & 403.7 & 24763 & 126 & 526.6 & 18985 \\
\hline 37 & 362.6 & 27572 & 82 & 458.5 & 21805 & 127 & 527.1 & 18967 \\
\hline 38 & 362.8 & 27557 & 83 & 458.7 & 21793 & 128 & 528.0 & 18934 \\
\hline 39 & 362.9 & 27545 & 84 & 459.1 & 21777 & 129 & 528.7 & 18908 \\
\hline 40 & 363.1 & 27530 & 85 & 459.5 & 21756 & 130 & 528.8 & 18906 \\
\hline 41 & 363.4 & 27512 & 86 & 459.9 & 21736 & 131 & 529.8 & 18871 \\
\hline 42 & 363.8 & 27480 & 87 & 460.2 & 21725 & 132 & 530.1 & 18859 \\
\hline 43 & 364.0 & 27465 & 88 & 460.5 & 21712 & 133 & 530.3 & 18853 \\
\hline 44 & 364.2 & 27450 & 89 & 461.0 & 21687 & 134 & 530.5 & 18846 \\
\hline 45 & 364.5 & 27427 & 90 & 461.1 & 21679 & 135 & 531.0 & 18826 \\
\hline
\end{tabular}


7.2. ENERGY LEVEL CALCULATIONS FOR TM ${ }^{3+}$ IN ALN

\begin{tabular}{|c|c|c|c|c|c|c|c|c|}
\hline$\overline{\text { No. }^{a}}$ & $\begin{array}{c}\lambda^{\mathrm{b}} \\
(\mathrm{nm})\end{array}$ & $\begin{array}{c}\mathrm{E}^{\mathrm{C}}{ }_{\text {obs }} \\
\left(\mathrm{cm}^{-1}\right)\end{array}$ & No. ${ }^{a}$ & $\begin{array}{c}\lambda^{\mathrm{b}} \\
(\mathrm{nm})\end{array}$ & $\begin{array}{c}\mathrm{E}^{\mathrm{c}}{ }_{o b s} \\
\left(\mathrm{~cm}^{-1}\right)\end{array}$ & ${ }$ No. $^{a}$ & $\begin{array}{c}\lambda^{\mathrm{b}} \\
(\mathrm{nm})\end{array}$ & $\begin{array}{c}\mathrm{E}^{\mathrm{C}}{ }_{\text {obs }} \\
\left(\mathrm{cm}^{-1}\right)\end{array}$ \\
\hline 136 & 532.0 & 18793 & 158 & 659.3 & 15163 & 180 & 777.0 & 12867 \\
\hline 137 & 533.1 & 18754 & 159 & 660.4 & 15139 & 181 & 777.5 & 12859 \\
\hline 138 & 544.0 & 18376 & 160 & 677.8 & 14750 & 182 & 778.0 & 12849 \\
\hline 139 & 544.4 & 18364 & 161 & 678.1 & 14744 & 183 & 779.0 & 12834 \\
\hline 140 & 545.9 & 18314 & 162 & 679.3 & 14716 & 184 & 780.4 & 12811 \\
\hline 141 & 546.0 & 18311 & 163 & 680.6 & 14689 & 185 & 780.4 & 12810 \\
\hline 142 & 546.2 & 18303 & 164 & 681.9 & 14661 & 186 & 783.9 & 12754 \\
\hline 143 & 546.9 & 18281 & 165 & 682.0 & 14658 & 187 & 784.2 & 12748 \\
\hline 144 & 548.5 & 18227 & 166 & 682.1 & 14656 & 188 & 785.1 & 12734 \\
\hline 145 & 548.4 & 18231 & 167 & 682.8 & 14642 & 189 & 786.1 & 12718 \\
\hline 146 & 548.7 & 18220 & 168 & 684.0 & 14616 & 190 & 788.0 & 12687 \\
\hline 147 & 549.3 & 18201 & 169 & 684.7 & 14601 & 191 & 804.5 & 12426 \\
\hline 148 & 623.2 & 16042 & 170 & 685.8 & 14577 & 192 & 804.7 & 12424 \\
\hline 149 & 623.8 & 16026 & 171 & 686.1 & 14570 & 193 & 805.0 & 12419 \\
\hline 150 & 624.3 & 16013 & 172 & 773.5 & 12924 & 194 & 805.3 & 12415 \\
\hline 151 & 626.4 & 15960 & 173 & 773.6 & 12923 & 195 & 806.5 & 12397 \\
\hline 152 & 649.1 & 15403 & 174 & 774.5 & 12908 & 196 & 807.6 & 12379 \\
\hline 153 & 650.7 & 15364 & 175 & 774.9 & 12902 & 197 & 808.8 & 12361 \\
\hline 154 & 651.9 & 15336 & 176 & 775.7 & 12888 & 198 & 808.9 & 12359 \\
\hline 155 & 652.0 & 15334 & 177 & 775.7 & 12887 & 199 & 811.3 & 12323 \\
\hline 156 & 654.6 & 15272 & 178 & 776.0 & 12884 & 200 & 812.1 & 12311 \\
\hline 157 & 654.7 & 15270 & 179 & 776.0 & 12883 & 201 & 813.6 & 12288 \\
\hline
\end{tabular}

${ }^{\mathrm{b}}$ Sequential number as indicated in figures 7.1 and 7.2 .

${ }^{\mathrm{b}}$ Experimental wave length in nanometers.

${ }^{\text {c }}$ Corresponding energy in vacuum wave numbers.

Table 7.1: Experimental transitions between $200 \mathrm{~nm}$ and $850 \mathrm{~nm}$ of $\mathrm{Tm}^{3+}$ in AlN, measured at $12 \mathrm{~K}$.

\subsection{Energy level calculations for $\mathbf{T m}^{3+}$ in AIN}

The $\mathrm{Tm}^{3+}\left(4 f^{12}\right)$ ion does not belong not the class of non-Kramers' ${ }^{1}$ ions. The number of $\mathrm{Tm}^{3+}$ crystal field levels expected for both the $\mathrm{C}_{3 v}$ and the $\mathrm{D}_{2}$ symmetry is listed in table 33 . This table shows into how many individual Stark levels the corresponding free ion ${ }^{2 S+1} L_{J}$ multiplet manifold is broken up. The notation for the assignment of each Stark levels follows the one given by Koster [301], using irreducible representations. Obviously, the number of expected Stark levels differs between $\mathrm{C}_{3 v}$ and $\mathrm{D}_{2}$ symmetry. Additionally, selected rules for Stark levels to Stark level transitions exist for both cases which are not listed here (see the selection rules for induced electric dipole and magnetic dipole transitions listed in Appendix 5, ref. [106]). The reason for listing both the $\mathrm{C}_{3 v}$ and $\mathrm{D}_{2}$ symmetry is that one might expect the $\mathrm{C}_{3 v}$ symmetry

${ }^{1}$ Kramers' theorem states that in odd electron systems the crystal field levels are at least doubly degenerate. 


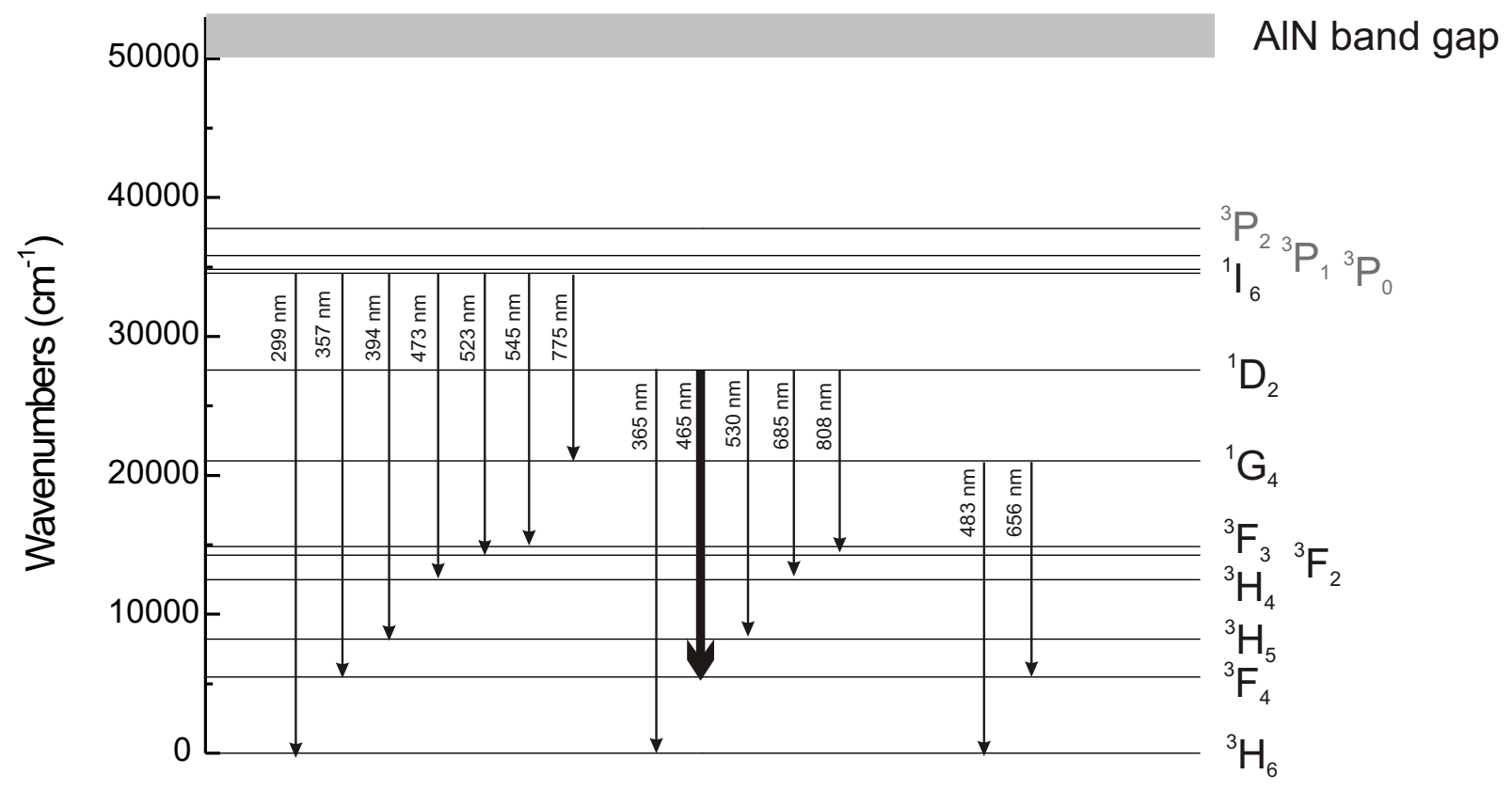

Figure 7.3: Experimental energy level diagram for $\mathrm{Tm}^{3+}$ in AlN. The calculated energy levels, which are not confirmed experimentally, are indicated as well.

referring to the lattice location studies for $\mathrm{Yb}^{3+}$ in this work, but one should keep in mind that lower symmetries than $\mathrm{C}_{3 v}$ are possible for different ions in the same host matrix as shown by Gruber et al. for the case of $\mathrm{GaN}[31,33,38]$.

In table 7.3 an assignment of luminescence lines to transitions between individual Stark levels belonging to certain multiplet manifolds is given for the case of $\mathrm{Tm}^{3+}$ in AlN. Initial multiplet manifolds are identified as ${ }^{1} I_{6}$ (initial Stark level energy position: $\left.33706 \mathrm{~cm}^{-1}\right),{ }^{1} D_{2}(27572$ $\mathrm{cm}^{-1}$ and $\left.27170 \mathrm{~cm}^{-1}\right)$, and ${ }^{1} G_{4}\left(20895 \mathrm{~cm}^{-1}\right)$. In section 5.1.3, the principle of searching repeating pattern in luminescence spectra was exemplarily introduced for the case of $\mathrm{Eu}^{3+}$ in AlN. The same approach has to be followed for $\mathrm{Tm}^{3+}$ in AlN. In Table 7.3 such pattern are listed for the ${ }^{1} H_{6}$ ground state as well as the first five excited levels ${ }^{3} F_{4},{ }^{3} H_{5},{ }^{3} H_{4},{ }^{3} F_{3}$ as well as ${ }^{3} F_{2}$. These multiplets, with the exception of ${ }^{3} F_{2}$, appear at least twice in the luminescence spectra. The positions of the initial Stark levels are indicated, they belong to the ${ }^{1} I_{6},{ }^{1} D_{2}$ and ${ }^{1} G_{4}$ levels. Transitions starting from the ${ }^{3} P_{J}$ levels above ${ }^{1} I_{6}$ were not detected and very likely decay nonradiatively to the latter one. Following the approach mentioned above, existing crystal field parameters were taken from the literature and fitted towards the observed splittings. During the fitting procedure the crystal field parameters were constrained to physically reasonable values by varying the charges at the nitrogen and aluminum atoms of the AlN host surrounding an isolated $\mathrm{Tm}^{3+}$ ion. Column no. 8 in Table 7.3 shows the calculated energy levels based on 


\begin{tabular}{lll}
\hline \hline & $\mathrm{C}_{3 v}$ & $\mathrm{D}_{2}$ \\
\hline${ }^{2 S+1} L_{J}$ level & Symmetry labels $\Gamma_{i}{ }^{\mathrm{a}}$ & Symmetry labels $\Gamma_{i}{ }^{\mathrm{a}}$ \\
\hline${ }^{3} P_{0},{ }^{1} S_{0}$ & $\Gamma_{1}$ & $\Gamma_{1}$ \\
${ }^{3} P_{1}$ & $\Gamma_{2}+\Gamma_{3}$ & $\Gamma_{2}+\Gamma_{3}+\Gamma_{3}$ \\
${ }^{3} P_{2},{ }^{1} D_{2},{ }^{3} F_{2}$ & $\Gamma_{1}+2 \Gamma_{3}$ & $2 \Gamma_{1}+\Gamma_{2}+\Gamma_{3}+\Gamma_{3}$ \\
${ }^{3} F_{3}$ & $\Gamma_{1}+2 \Gamma_{2}+2 \Gamma_{3}$ & $\Gamma_{1}+2 \Gamma_{2}+2 \Gamma_{3}+2 \Gamma_{3}$ \\
${ }^{3} F_{4},{ }^{3} F_{4},{ }^{1} G_{4}$ & $2 \Gamma_{1}+\Gamma_{1}+3 \Gamma_{3}$ & $3 \Gamma_{1}+2 \Gamma_{2}+2 \Gamma_{3}+2 \Gamma_{3}$ \\
${ }^{3} H_{5}$ & $\Gamma_{1}+2 \Gamma_{1}+4 \Gamma_{3}$ & $2 \Gamma_{1}+3 \Gamma_{2}+3 \Gamma_{3}+3 \Gamma_{3}$ \\
${ }^{3} H_{6},{ }_{6}$ & $3 \Gamma_{1}+2 \Gamma_{1}+4 \Gamma_{3}$ & $4 \Gamma_{1}+3 \Gamma_{2}+3 \Gamma_{3}+3 \Gamma_{3}$ \\
\hline \hline
\end{tabular}

${ }^{a}$ Notation according to Koster [301]. Corresponding Mulliken symbols additionally showing the degeneracy of each Stark level are listed in Ref. [106].

Table 7.2: Full-rotational group compatibility table for $\mathrm{Tm}^{3+}$ energy levels under symmetry $\mathrm{C}_{3 v}$ and $\mathrm{D}_{2}$ [106]. The number of Stark levels each ${ }^{2 S+1} L_{J}$ manifold is broken up into under the given point group symmetry corresponds to the number of irreducible representations.

the crystal field for symmetry $\mathrm{C}_{3 v}$. Good fits were obtained for the crystal field parameters $B_{20}=-31.8, B_{40}=-556, B_{43}=-650, B_{60}=754, B_{63}=-353$ and $B_{66}=403$ with an rms value of $10 \mathrm{~cm}^{-1}$ for 75 experimental Stark levels and 6 crystal field parameters. Additionally the values of the crystal field quantum number $\mu$ used as the symmetry label are indicated. These calculated labels could not be confirmed experimentally.

The good agreement between experimental and calculated energy levels suggests that the assumption of $\mathrm{Tm}^{3+}$ ions on $\mathrm{C}_{3 v}$ cationic (Al) sites is reasonable. In the above list, only 75 out of 201 luminescence lines are assigned and further calculations are necessary to obtain an almost complete energy level diagram. A rigid analysis requires that up to $90 \%$ of the experimental energy levels can be assigned. The main problem in the case of $\mathrm{Tm}^{3+}$ in $\mathrm{AlN}$ is that a large number of transitions were detected which cannot be explained by the energy level diagram. Quite the contrary, these transitions must be attributed to phonon replica which indeed accompany almost each intense transition. Typical examples are (compare figures 7.1 and 7.2) lines nos. 10-16 (corresponding zero-phonon transition: $\left.{ }^{1} I_{6} \rightarrow{ }^{3} H_{6}\right), 71-81\left({ }^{1} I_{6} \rightarrow{ }^{3} H_{5}\right)$ and others.

2 root mean square:

$$
\sigma=\left[\frac{\sum_{N}\left(E_{\exp , N}-E_{c a l c, N}\right)^{2}}{N-P}\right]
$$

where $\mathrm{N}$ is the number of experimental transitions between energy levels with the energy difference $E_{\text {exp }}$; $E_{\text {calc }}$ are the calculated energy differences and $P$ the number of parameters varied during fitting. 
CHAPTER 7. CRYSTAL FIELD ANALYSIS FOR TM ${ }^{3+}\left(4 F^{12}\right)$ AND GD ${ }^{3+}\left(4 F^{7}\right)$ IN ALN

\begin{tabular}{|c|c|c|c|c|c|c|c|c|}
\hline $\begin{array}{l}\text { Level }^{\mathrm{b}} \\
2 S+1 L_{J}\end{array}$ & Trans. ${ }^{\mathrm{c}}$ & $\begin{array}{l}\mathrm{E}_{\exp }{ }^{\mathrm{d}} \\
\left(\mathrm{cm}^{-1}\right)\end{array}$ & Trans. $^{c}$ & $\begin{array}{l}\Delta \mathrm{E}_{e x p}{ }^{\mathrm{d}} \\
\left(\mathrm{cm}^{-1}\right)\end{array}$ & Trans. $^{c}$ & $\begin{array}{l}\Delta \mathrm{E}_{\text {exp }}{ }^{\mathrm{d}} \\
\left(\mathrm{cm}^{-1}\right)\end{array}$ & $\begin{array}{c}\mathrm{E}^{\mathrm{e}}{ }_{\text {calc }} \\
\left(\mathrm{cm}^{-1}\right)\end{array}$ & $2 \mu^{\mathrm{f}}$ \\
\hline & & ${ }^{1} I_{6}$ & & ${ }^{1} D_{2}$ & & ${ }^{1} G_{4}$ & & \\
\hline${ }^{3} H_{6}$ & $2 \rightarrow 2$ & 0 & $37 \rightarrow 37$ & 0 & $108 \rightarrow 108$ & 0 & 1 & 0 \\
\hline \multirow[t]{8}{*}{$(208)$} & $2 \rightarrow 3$ & 46 & $37 \rightarrow 40$ & 42 & $108 \rightarrow 111$ & 46 & 45 & 0 \\
\hline & $2 \rightarrow 4$ & 99 & $37 \rightarrow 42$ & 96 & & & 87 & 2 \\
\hline & $2 \rightarrow 5$ & 106 & $37 \rightarrow 43$ & 107 & $108 \rightarrow 112$ & 109 & 109 & 0 \\
\hline & $2 \rightarrow 6$ & 155 & $37 \rightarrow 46$ & 155 & $108 \rightarrow 115$ & 157 & 156 & 2 \\
\hline & $2 \rightarrow 7$ & 185 & $37 \rightarrow 48$ & 190 & $108 \rightarrow 116$ & 187 & 194 & 0 \\
\hline & $2 \rightarrow 9$ & 343 & $37 \rightarrow 50$ & 346 & $108 \rightarrow 120$ & 348 & 342 & 2 \\
\hline & & & & & & & 354 & 0 \\
\hline & & & & & & & 375 & 2 \\
\hline \multirow{6}{*}{$\begin{array}{c}{ }^{3} F_{4} \\
(5661)\end{array}$} & $2 \rightarrow 19$ & 5491 & $51 \rightarrow 90$ & 5491 & $108 \rightarrow 152$ & 5493 & 5502 & 0 \\
\hline & $2 \rightarrow 20$ & 5530 & $51 \rightarrow 91$ & 5530 & $108 \rightarrow 153$ & 5531 & 5515 & 2 \\
\hline & $2 \rightarrow 21$ & 5558 & $51 \rightarrow 92$ & 5558 & $108 \rightarrow 154$ & 5559 & 5558 & 0 \\
\hline & $2 \rightarrow 24$ & 5623 & $51 \rightarrow 96$ & 5622 & $108 \rightarrow 156$ & 5623 & 5622 & 0 \\
\hline & $2 \rightarrow 28$ & 5732 & $51 \rightarrow 100$ & 5731 & $108 \rightarrow 158$ & 5732 & 5731 & 2 \\
\hline & $2 \rightarrow 29$ & 5755 & $51 \rightarrow 102$ & 5754 & $108 \rightarrow 159$ & 5556 & 5754 & 2 \\
\hline \multirow{7}{*}{$\begin{array}{c}{ }^{3} H_{5} \\
(8304)\end{array}$} & $2 \rightarrow 57$ & 8197 & $51 \rightarrow 126$ & 8192 & & & 8208 & 2 \\
\hline & $2 \rightarrow 58$ & 8206 & $51 \rightarrow 127$ & 8203 & & & 8210 & 0 \\
\hline & $2 \rightarrow 61$ & 8269 & $51 \rightarrow 130$ & 8265 & & & 8249 & 2 \\
\hline & $2 \rightarrow 62$ & 8322 & $51 \rightarrow 133$ & 8318 & & & 8318 & 2 \\
\hline & $2 \rightarrow 64$ & 8344 & $51 \rightarrow 134$ & 8342 & & & 8331 & 0 \\
\hline & & & $51 \rightarrow 136$ & 8377 & & & 8384 & 0 \\
\hline & $2 \rightarrow 69$ & 8447 & $51 \rightarrow 137$ & 8445 & & & 8428 & 2 \\
\hline \multirow{6}{*}{$\begin{array}{c}{ }^{3} H_{4} \\
(12563)\end{array}$} & $2 \rightarrow 103$ & 12426 & $51 \rightarrow 161$ & 12427 & & & 12431 & 2 \\
\hline & $2 \rightarrow 104$ & 12481 & $51 \rightarrow 163$ & 12480 & & & 12481 & 0 \\
\hline & $2 \rightarrow 105$ & 12532 & $51 \rightarrow 166$ & 12514 & & & 12503 & 2 \\
\hline & $2 \rightarrow 106$ & 12553 & $51 \rightarrow 168$ & 12554 & & & 12560 & 2 \\
\hline & $2 \rightarrow 107$ & 12599 & $51 \rightarrow 171$ & 12600 & & & 12600 & 0 \\
\hline & & & & & & & 12720 & 0 \\
\hline \multirow{5}{*}{$\begin{array}{c}{ }^{3} F_{3} \\
(14800)\end{array}$} & $2 \rightarrow 127$ & 14739 & $51 \rightarrow 191$ & 14744 & & & 14738 & 0 \\
\hline & $2 \rightarrow 129$ & 14798 & $51 \rightarrow 196$ & 14791 & & & 14785 & 2 \\
\hline & $2 \rightarrow 130$ & 14814 & $51 \rightarrow 197$ & 14809 & & & 14801 & 0 \\
\hline & $2 \rightarrow 131$ & 14817 & $51 \rightarrow 198$ & 14811 & & & 14817 & 0 \\
\hline & $2 \rightarrow 132$ & 14847 & $51 \rightarrow 199$ & 14847 & & & 14847 & 2 \\
\hline \multirow{3}{*}{$\begin{array}{c}{ }^{3} F_{2} \\
(15366)\end{array}$} & $2 \rightarrow 139$ & 15342 & & & & & 15334 & 2 \\
\hline & $2 \rightarrow 140$ & 15395 & & & & & 15405 & 0 \\
\hline & $2 \rightarrow 141$ & 15402 & & & & & 15408 & 2 \\
\hline
\end{tabular}

${ }^{\text {a }}$ Spectra obtained at $12 \mathrm{~K}$.

b ${ }^{2 S+1} L_{J}$ multiplet manifolds for $\mathrm{Tm}^{3+}\left(4 f^{12}\right)$, the number in parentheses indicates the calculated centroid for each manifold.

${ }^{c}$ Transitions as assigned in the Figures 7.1 and 7.2 .

${ }^{\mathrm{d}}$ Corresponding energy difference between transitions.

${ }^{\mathrm{e}}$ Calculated splitting based on $\mathrm{Tm}^{3+}$ in $\mathrm{C}_{3 v}$ sites: $B_{20}=-31.8, B_{40}=-556, B_{43}=-650, B_{60}=754$, $B_{63}=-353, B_{66}=403$.

f Symmetry label $2 \mu=0,2 \mu=2$, calculated using the $B_{n m}$ parameters in footnote e.

134 Table 7.3: Energy levels of the four lowest multiplets of $\mathrm{Tm}^{3+}\left(4 f^{12}\right)$ in AlN. 


\subsection{EXPERIMENTAL CL STARK LEVEL SPECTRA OF GD ${ }^{3+}$ IN ALN}

Such phonon side bands were also detected in the case of $\mathrm{AlN}: \mathrm{Eu}^{3+}$ (see chapter 5.1.3) and AlN: $\mathrm{Gd}^{3+}$ and will be discussed in chapter 7.6 .

\subsection{Experimental CL Stark level spectra of $\mathbf{G d}^{3+}$ in AIN}

The survey scan presented in Figure 5.2 suggests that both the ${ }^{6} P_{7 / 2}$ and the ${ }^{6} P_{5 / 2}$ levels are the only ones detectable. However, after carefully scanning the wavelength range of $200 \mathrm{~nm}$ to $800 \mathrm{~nm}$, additional weak transitions could be found, their intensity being by 2 to 3 orders of magnitude weaker than the one of the strongest transitions, ${ }^{6} P_{7 / 2} \rightarrow{ }^{8} S_{7 / 2}$. All the spectra are shown in figure 7.4. Based on the energy level diagram of $\mathrm{Gd}^{3+}$ and the fact that the ground state is unresolved, the transitions detected at $300 \mathrm{~K}$ were assigned directly to the individual
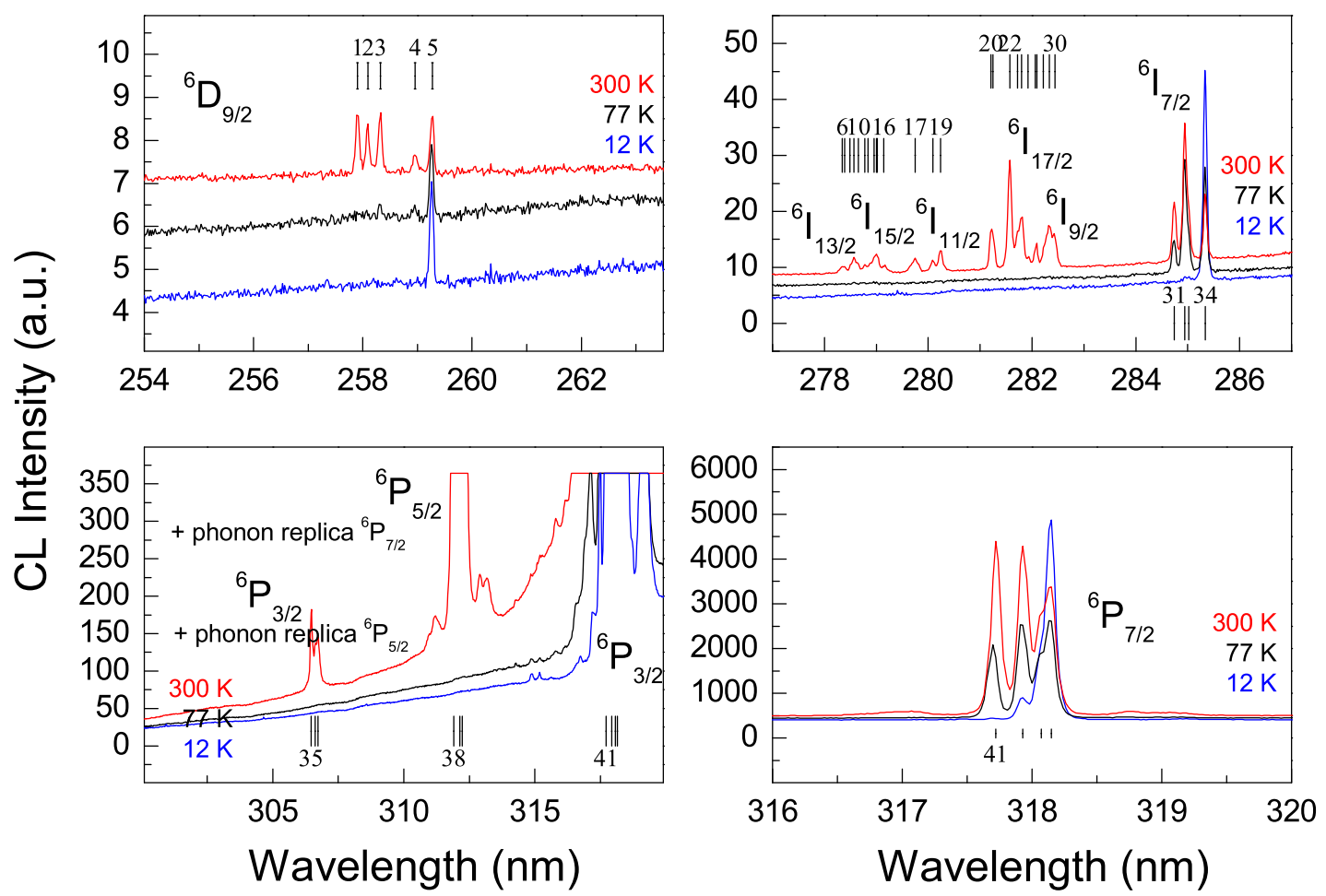

Figure 7.4: High resolution spectra of excited levels of $\mathrm{Gd}^{3+}$ in AlN. All transitions go to the ground state ${ }^{8} S_{7 / 2}$, which was not resolved experimentally. The spectra were recorded at $12 \mathrm{~K}, 77 \mathrm{~K}$ and $300 \mathrm{~K}$. The thermal population is clearly visible for all transitions. Individual transitions are numbered consecutively (compare table 7.5 ) 
Stark levels of the ${ }^{6} D_{9 / 2},{ }^{6} \mathrm{P}_{J^{\prime} / 2, J^{\prime}=7,5,3}$ and ${ }^{6} \mathrm{I}_{J^{\prime} / 2, J^{\prime}=7,9,11,13,15,17}$ multiplets. At $12 \mathrm{~K}$ only the lowest Stark levels of the ${ }^{6} D_{9 / 2}$ and ${ }^{6} I_{7 / 2}$ multiplets could be detected. It is obvious that most Stark levels are thermally populated at $300 \mathrm{~K}$, and again the integral intensity of each multiplet increases with increasing temperature, similar to the behavior observed for the ${ }^{6} P_{7 / 2} \rightarrow{ }^{8} S_{7 / 2}$ transition. The complete list of energy levels is given in table 7.5. The illustration follows the one used by Gruber et al. [31, 33, 38]. Due to the weak crystal field splitting for the case of $\mathrm{Gd}^{3+}$ in AlN, the resolution of the setup has almost reached its upper limit.

It is worth mentioning that for $\mathrm{Gd}^{3+}$ in AlN the luminescence Stark level spectra are very different from the ones of other lanthanides. Usually luminescence spectra taken at low temperatures show pattern representing (a part) of the final multiplets of the transitions. For the case of $\mathrm{Gd}^{3+}$ the spectra recorded at $300 \mathrm{~K}$ very much resemble those taken in absorption spectroscopy. Furthermore, each luminescence line represents transitions between all individual Stark levels of the ground state and a certain level of the excited multiplet. Weighing the integral intensities with the fractional thermal population would indeed allow an intensity analysis. The following chapter will, however, deal with the crystal field analysis only, but an intensity analysis is being

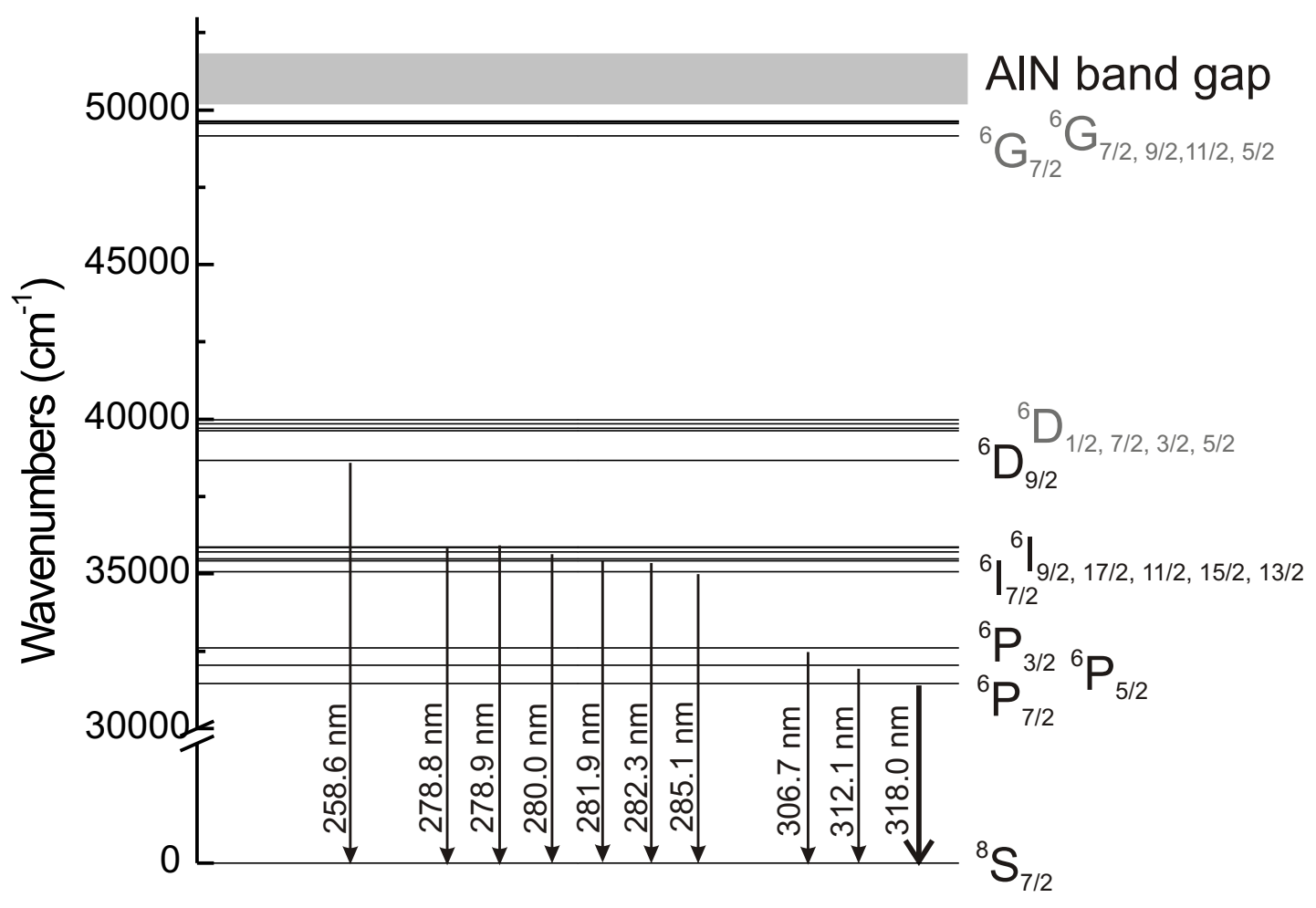

Figure 7.5: Experimental energy level diagram for $\mathrm{Gd}^{3+}$ in AlN. Additionally, calculated energy levels which are not confirmed experimentally are indicated as well. 
prepared.

\subsection{Energy level calculations for $\mathbf{G d}^{3+}$ in $\mathrm{AIN}$}

Energy level calculations for $\mathrm{Gd}^{3+}$ follow the same approach as discussed above for the case of $\mathrm{Tm}^{3+}$ in AlN. The main difference is that $\mathrm{Gd}^{3+}\left(4 f^{7}\right)$ is a Kramers ion, its crystal field Stark levels are at least doubly degenerate, disregarding the symmetry of the crystal field. Table 7.4 shows the expected number of Stark levels for $\mathrm{Gd}^{3+}$ ions in sites with the symmetries $\mathrm{C}_{3 v}$ and $\mathrm{D}_{2}$. The most important multiplet manifolds as regards the experimental observations discussed in the preceding chapter are listed. Obviously, one may not distinguish between the two symmetries on the basis of the number of crystal field levels; on the contrary, even for symmetries lower than $\mathrm{D}_{2}$ the number of Stark levels will not increase. This is a known problem with odd electron (Kramers) systems and may, in principle, only be overcome by applying polarized absorption or excitation spectroscopy.

Existing sets of crystal field parameters were self-consistently varied within reasonable ranges. Diagonalization of the energy matrix with dimension $3432^{2}$ for $\mathrm{Gd}^{3+}$ may last very long; thus the set of eigenfunctions was truncated to the 25 with lowest energy eigenvalues. Table 7.5 on the following pages summarizes the complete outcome of these calculations. In the third

\begin{tabular}{lll}
\hline \hline & $\mathrm{C}_{3 v}$ & $\mathrm{D}_{2}$ \\
\hline${ }^{2 S+1} L_{J}$ level & Symmetry labels $\Gamma_{i}{ }^{\mathrm{a}}$ & Symmetry labels $\Gamma_{i}{ }^{\mathrm{a}}$ \\
\hline${ }^{6} D_{1 / 2}$ & $\Gamma_{4}$ & $\Gamma_{5}$ \\
${ }^{6} D_{3 / 2},{ }^{6} P_{3 / 2}$ & $\Gamma_{4}+\Gamma_{5,6}$ & $2 \Gamma_{5}$ \\
${ }^{6} D_{5 / 2},{ }^{6} P_{5 / 2}$ & $2 \Gamma_{4}+\Gamma_{5,6}$ & $3 \Gamma_{5}$ \\
${ }^{6} I_{7 / 2},{ }^{8} S_{7 / 2},{ }^{6} D_{7 / 2}$ & $3 \Gamma_{4}+\Gamma_{5,6}$ & $4 \Gamma_{5}$ \\
${ }^{6} I_{9 / 2},{ }^{6} D_{9 / 2}$ & $3 \Gamma_{4}+2 \Gamma_{5,6}$ & $5 \Gamma_{5}$ \\
${ }^{6} I_{11 / 2}$ & $4 \Gamma_{4}+2 \Gamma_{5,6}$ & $6 \Gamma_{5}$ \\
${ }^{6} I_{13 / 2}$ & $5 \Gamma_{4}+2 \Gamma_{5,6}$ & $7 \Gamma_{5}$ \\
${ }^{6} I_{15 / 2}$ & $3 \Gamma_{4}+3 \Gamma_{5,6}$ & $8 \Gamma_{5}$ \\
${ }^{6} I_{17 / 2}$ & $6 \Gamma_{4}+3 \Gamma_{5,6}$ & $9 \Gamma_{5}$ \\
\hline \hline
\end{tabular}

${ }^{a}$ Notation according to Koster [301]. Corresponding Mulliken symbols additionally showing the degeneracy of each Stark level are listed in Ref. [106].

Table 7.4: Full-rotational group compatibility table for $\mathrm{Gd}^{3+}$ energy levels under symmetries $\mathrm{C}_{3 v}$ and $\mathrm{D}_{2}$ [106]. The number of Stark levels each ${ }^{2 S+1} L_{J}$ manifold is broken up into under the given point group symmetry corresponds to the number of irreducible representations. 
row the positions of the energy levels extracted in wavelengths from figure 7.4 , and numbered (second row) as indicated there, are listed. These experimental values are converted to vacuum energy wavenumbers following the approach of chapter 4.1.7. An assignment of the Stark levels to multiplet manifolds is given in the first row of Table 7.5 , the value in brackets denotes the calculated barycenter of the manifold. The last two rows in the table give the calculated crystal field quantum numbers $\mu$ as well as the Stark level eigenfunctions in terms of $L S J$ eigenfunctions of the free ion. This notation is commonly adopted in the literature and indicates the mixing of wavefunctions with different $J$ quantum numbers by the crystal field. It should be kept in mind at this point that the free ion wavefunctions are themselves linear combinations of wavefunctions with different quantum numbers $L$ and $S$ but the same $J$. $J$-mixing, however, only appears in the presence of a crystal field. It is also obvious that $J$-mixing is stronger in case the multiplets are closely spaced as can be seen from the free-ion mixture of some of the ${ }^{6} I_{15 / 2}$ and ${ }^{6} I_{13 / 2}$ Stark levels. In such a case the intermediate coupling approximation is not valid any longer (it is said to break down) and the remaining selection rules for induced electric dipole radiation in the case of intermediate coupling become invalid (compare Table 3.2)

The final crystal field parameters for symmetry $\mathrm{C}_{3 v}$ obtained during the fitting procedure are $B_{20}=-211, B_{40}=-1691, B_{43}=-1063, B_{60}=655.5, B_{63}=-188.4$, and $B_{66}=293.7$. The rms value between 46 experimental to calculated energy levels and 6 crystal field parameters is $8 \mathrm{~cm}^{-1}$. The assumption of a $\mathrm{C}_{3 v}$ symmetry for $\mathrm{Gd}^{3+}$ in the AlN host is therefore reasonable. The calculations predict a ground state splitting of only $0.3 \mathrm{~cm}^{-1}$, which explains why it is experimentally not resolved.

\subsection{Branching ratios and the $\Omega_{2}$ parameter}

The preceding crystal field analysis for $\mathrm{Tm}^{3+}\left(4 f^{12}\right)$ allowed to identify the symmetry of the $\mathrm{Tm}^{3+}$ ions in the AlN host. Another important outcome of the calculations is the identification of the most dominant transitions appearing in the CL spectra. These results can be used to understand the time-resolved luminescence spectra discussed in chapter 5.1.4 (see figure 5.9), indicating that the strong blue luminescence at around $465 \mathrm{~nm}$ is attributable to the ${ }^{1} D_{2} \rightarrow{ }^{3} F_{4}$ transition in combination with non-radiative deexcitation of the ${ }^{1} I_{6}$ level, thereby effectively populating the ${ }^{3} F_{4}$ level. The luminescence spectrum of $\mathrm{Tm}^{3+}$-implanted AlN at $300 \mathrm{~K}$ (figure 5.9 is almost completely dominated by transitions starting from the ${ }^{1} D_{2}$ level, namely ${ }^{1} D_{2} \rightarrow{ }^{3} F_{4}(465 \mathrm{~nm}),{ }^{1} D_{2} \rightarrow{ }^{3} H_{4}(484 \mathrm{~nm}),{ }^{1} D_{2} \rightarrow{ }^{3} F_{3}(775 \mathrm{~nm})$, and ${ }^{1} D_{2} \rightarrow{ }^{3} F_{2}(802 \mathrm{~nm})$. The relative experimental intensities of these transitions, listed in table 7.5 and representing the branching ratios (see equation 3.41), can be compared to values reported in the literature. Es- 


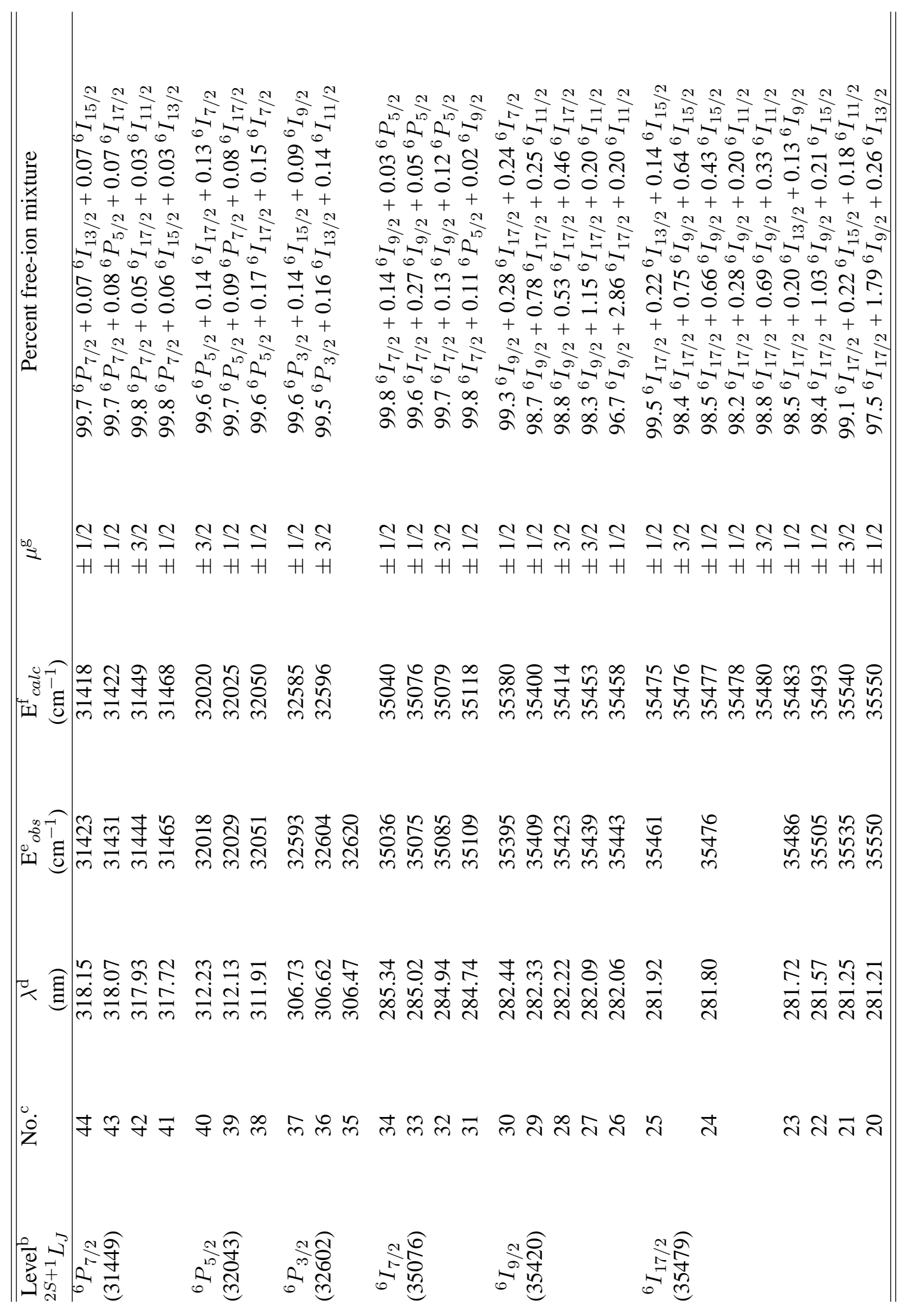


CHAPTER 7. CRYSTAL FIELD ANALYSIS FOR TM ${ }^{3+}\left(4 F^{12}\right)$ AND GD $^{3+}\left(4 F^{7}\right)$ IN ALN

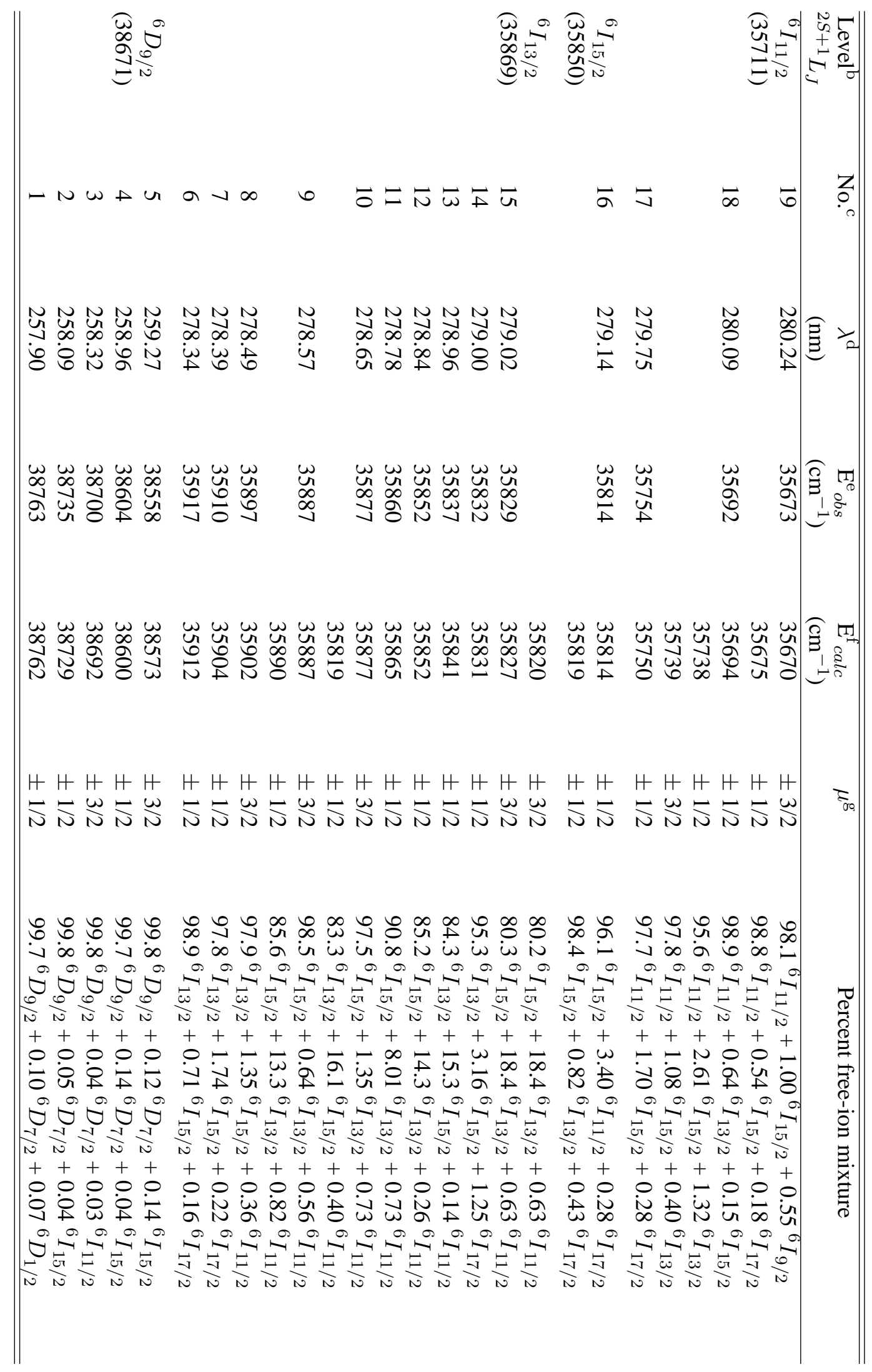




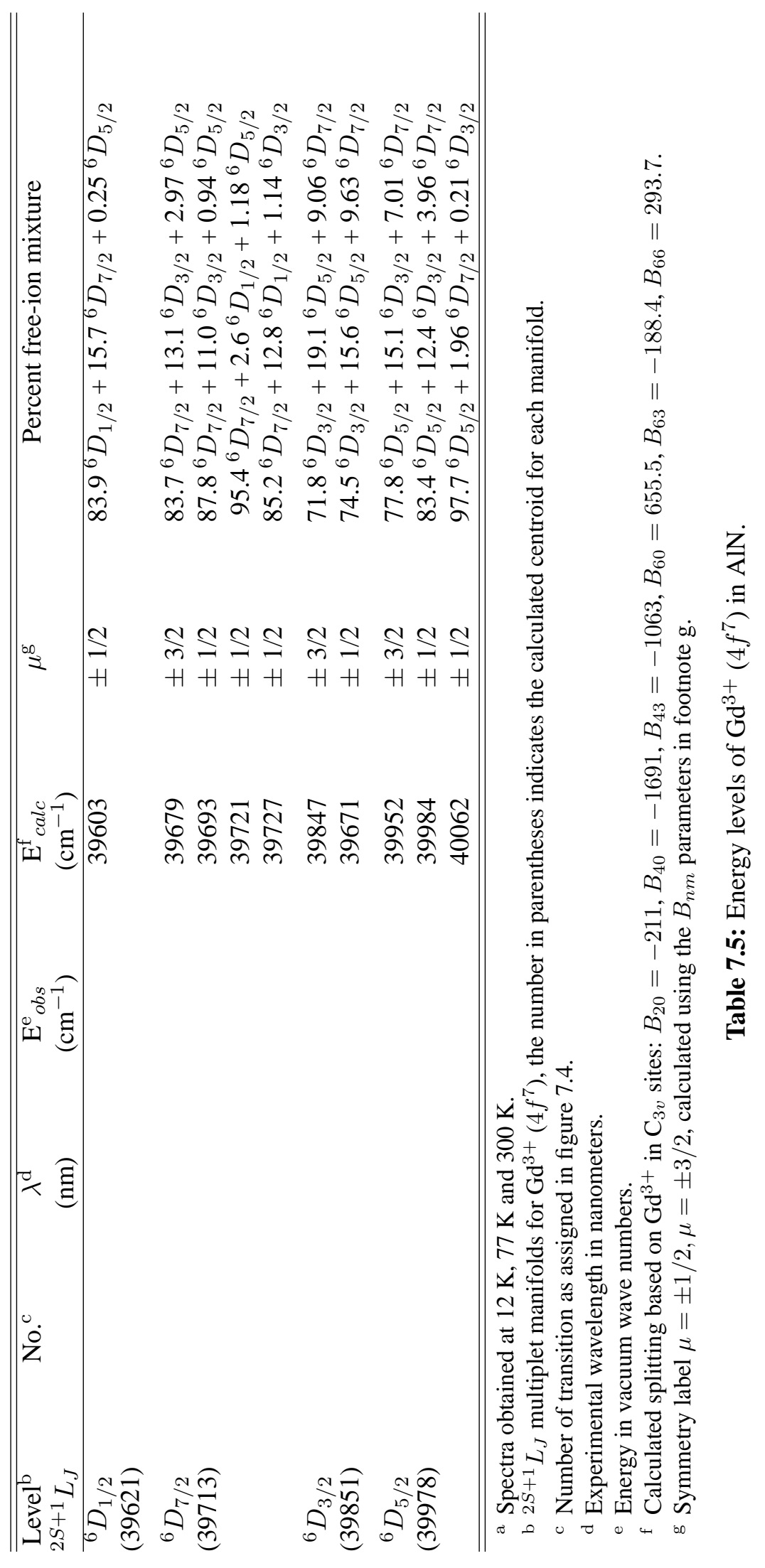


pecially with respect to the relative intensities of the ${ }^{1} D_{2} \rightarrow{ }^{3} F_{4}$ and ${ }^{1} D_{2} \rightarrow{ }^{3} H_{6}$ transition there has been considerable interest to understand and modify this intensity ratio. This is due to the application of $\mathrm{Tm}^{3+}$ in upconversion devices [300, 152, 302, 153, 303, 251, 154, 155, 156, 157], where infrared light can be converted to visible (e.g. ${ }^{1} D_{2} \rightarrow{ }^{3} F_{4}$ ) or ultra violet (e.g. ${ }^{1} D_{2} \rightarrow{ }^{3} H_{6}$ ) light when the ${ }^{1} D_{2}$ level is involved in the upconversion process [152, 302, 153, 303, 251]. Systematic studies of Tanabe et al. [152, 251] and others for $\mathrm{Tm}^{3+}$ in glasses have shown that the ratio of the branching ratios of the ${ }^{1} D_{2} \rightarrow{ }^{3} F_{4}$ (this branching ratio which will be called $\beta_{24}$ in the following) and the ${ }^{1} D_{2} \rightarrow{ }^{3} H_{6}\left(\beta_{26}\right)$ transitions is largely influenced be the ligand field.

In order to compare the experimental branching ratios shown in table 7.5 with the predictions for $\mathrm{Tm}^{3+}$ in AlN, a few more considerations are necessary. Taking the transition probability for multiplet to multiplet transition (equation 3.37) and the parametrization for intensities between transitions as given by Axe (equation 3.32), one may express the transition probability for induced electric dipole radiation in the case of emission as follows:

$$
\begin{aligned}
A(i \rightarrow f) & =\frac{64 \pi^{4} \bar{\nu}^{3} e^{2}}{27 h[J]}\left[n\left(n^{2}+2\right)^{2} \sum_{\lambda=2,4,6} \Omega_{\lambda} \mid\left\langle l^{N} \gamma J|| U^{(\lambda)}|| l^{N} \gamma^{\prime} J^{\prime}\right\rangle\right] \\
& \equiv \sum_{\lambda=2,4,6} A^{\prime(\lambda)} \Omega_{\lambda}
\end{aligned}
$$

where everything that can be calculated is summed up in the parameters $A^{\prime(\lambda)}$. A graphical visualization of the reduced matrix elements $\left\langle f^{121} D_{2}|| U^{(\lambda)} \| f^{12} L^{\prime} S^{\prime} J^{\prime}\right\rangle$ is given in the upper graph of figure 7.6, the lower graph shows the calculated $A^{\prime(\lambda)}$ parameters. The wavelength dependent refractive index $n(\lambda)$, parametrized in Sellmeier-type form (cf. equation 3.36) was taken from the literature [304] $(A=1.00, B=3.12, C=138.0)$. Energy differences $\nu$ are taken from the crystal field calculations (calculated barycenters). All transitions from ${ }^{1} D_{2}$, except ${ }^{1} D_{2} \rightarrow{ }^{1} G_{4}$,

\begin{tabular}{cccccccc}
\hline \hline${ }^{1} D_{2} \rightarrow$ & ${ }^{3} H_{6}$ & ${ }^{3} F_{4}$ & ${ }^{3} H_{5}$ & ${ }^{3} H_{4}$ & ${ }^{3} F_{3}$ & ${ }^{3} F_{2}$ & ${ }^{1} G_{4}$ \\
\hline $\mathrm{I}_{\text {rel.,exp. }}{ }^{\mathrm{a}}$ & $<0.02^{\mathrm{c}}$ & 1 & $<0.02^{\mathrm{c}}$ & 0.08 & 0.06 & $<0.02^{\mathrm{c}}$ & - \\
$\mathrm{I}_{\text {rel.,calc. }}{ }^{\mathrm{c}}$ & 0 & 1 & 0 & 0.07 & 0.05 & 0.02 & 0.03 \\
\hline \hline
\end{tabular}

${ }^{\mathrm{a}}$ Experimental relative intensities taken from data of figure 5.2 at $300 \mathrm{~K}$, relative to experimental intensity of the ${ }^{1} D_{2} \rightarrow{ }^{3} F_{4}$ transition.

${ }^{\text {b }}$ Calculated relative intensities; relative to the calculate intensity of the ${ }^{1} D_{2} \rightarrow{ }^{3} F_{4}$ transition, on basis of $U^{2}$ $\left(U^{4}=0, U^{6}=0\right)$.

${ }^{\mathrm{c}}$ Estimated upper intensity limit, because the transitions overlap with the transition from ${ }^{1} I_{6}$ and corresponding phonon side bands.

Table 7.6: Experimental and calculated relative intensities for the ${ }^{1} D_{2} \rightarrow{ }^{2 S+1} L_{J}$ transition of $\mathrm{Tm}^{3+}$ in AlN. 
which falls into the infrared region of the electromagnetic spectrum $\left(\sim 6500 \mathrm{~cm}^{-1}\right)$, are experimentally confirmed. Magnetic dipole contributions to the transition probabilities are neglected, although contributions different from zero exist for the ${ }^{1} D_{2} \rightarrow{ }^{3} F_{3}$ and ${ }^{1} D_{2} \rightarrow{ }^{3} F_{2}$ transitions according to the corresponding selection rules (cf. table 3.2).

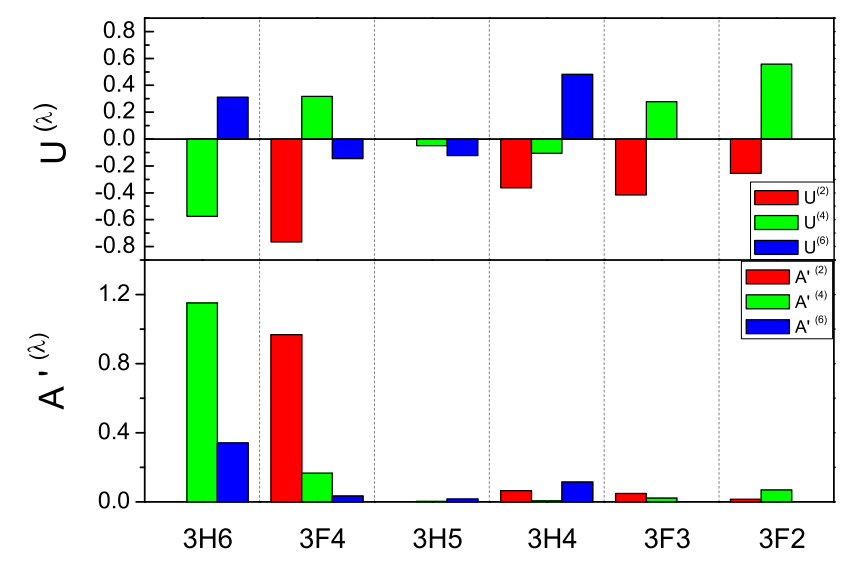

Figure 7.6: Reduced matrix elements $\left\langle{ }^{1} D_{2}|| U^{((\lambda))} \mid{ }^{2 S+1} L_{J}\right\rangle$ (in units $\left.\mathrm{cm}^{-1}\right)$ and transition probabilities $A^{\prime(\lambda)}$ (here: $A^{\prime(\lambda)}=\left\|U^{\lambda}\right\|^{2} \bar{\nu}^{3}$ in units of $10^{18} \mathrm{~s}^{-1} \mathrm{~cm}^{-2}$ ) for transitions from the ${ }^{1} \mathrm{D}_{2}$ level of $\mathrm{Tm}^{3+}$ (cf. figure 3.1.8. For further details see text.

Comparing the experimental branching ratios $\beta_{26} / \beta_{24}$ and the calculated $A^{\prime(\lambda)}$ parameters as indicated in figure 7.6 , it is evident that $\Omega_{2}$ must be the dominating intensity parameter in this case. The calculated parameters $A^{\prime(2)}$ are normalized with respect to $A^{\prime(2)}\left({ }^{1} D_{2} \rightarrow{ }^{3} F_{4}\right)$ and compared the relative experimental values in table 7.5 . Although the overall agreement is quite good, still a reservation has to be considered: the ${ }^{1} D_{2}$ level is definitely not equally thermally populated at $300 \mathrm{~K}$ as required for the validity of equation 7.5 .

Based on the above calculations, an $\Omega_{2}$ parameter of $\sim 250 \cdot 10^{-20} \mathrm{~cm}^{-2}$ would be necessary to reproduce the lifetime of the ${ }^{1} D_{2}$ level at $300 \mathrm{~K}$ as indicated in figure 5.9, a value by far larger than the ones commonly reported in the literature (compare table 20 in ref. [107]). This is an indication of a relatively low quantum efficiency, probably not more than 5-15\%. One possible explanation is non-radiative deexcitation due to multi-phonon emission. An efficient backtransfer process to the host may, however, be present as well. The suggested upper limit for the quantum efficiency is much lower than e.g. values of $\sim 80 \%$ estimated for selected transitions of $\mathrm{Sm}^{3+}$ in $\mathrm{GaN}$ [32, 33].

At the beginning of this chapter it was mentioned that several authors have reported on ratios of the branching ratios $\beta_{26} / \beta_{24}$ and it was deduced from experimental data that this ratio is dominated by the asymmetry of the ligand field. Tanabe et al. [152, 251] showed that $\beta_{26} / \beta_{24}$ can be significantly altered by replacing the nearest neighbor ligands in $\mathrm{Tm}^{3+}$-doped glasses 
with more or less ionic species. The authors mention that both $\Omega_{4}$ and $\Omega_{6}$ are large in case the covalency of the bonding is raised. This is, however, in contradiction to the observed behavior for $\mathrm{Tm}^{3+}$ in AlN. There is absolutely no evidence of a low symmetry, and $\mathrm{N}-\mathrm{Ln}^{3+}$-bonds are expected to be less ionic than the corresponding oxygen- oder fluorine bonds.

Based on the above observations, one may ask whether the result of a dominating $\Omega_{2}$ parameter for $\mathrm{Tm}^{3+}$ in AlN may be applied to $\mathrm{Gd}^{3+}$ in AlN. Doing so, this would indeed explain the rather weak intensities for transitions from the ${ }^{6} I_{J}$ levels as shown in figure 7.4 , because the rank-2 squared reduced matrix elements for transitions from these levels to the ground state are all zero (compare figure 3.1.8. However, because the transition probability of the ${ }^{6} P_{7 / 2} \rightarrow{ }^{8} S_{7 / 2}$ transition, for which lifetime measurements were performed (see figure 5.4), only depends on the $\Omega_{2}$ parameter; consequently observations for $\Omega_{2}$ in case of $\mathrm{Tm}^{3+}$ in AlN cannot be compared to $\mathrm{Gd}^{3+}$ in AlN. On the other hand, following the same approach as above, the $\Omega_{2}$ parameter, assuming a quantum efficiency of 1 , can be calculated. The results yield a value of $\Omega_{2} \approx$ $9.5 \cdot 10^{-20} \mathrm{~cm}^{-2}$, which is only slightly above the values reported in the literature (cf. table 15 in ref. [107]), which range from 0.32 to $7.27 \cdot 10^{-20} \mathrm{~cm}^{-2}$. This result principally supports the conclusion of a non-radiative energy transfer as the origin of the low quantum efficiency for $\mathrm{Tm}^{3+}$ in AlN.

\subsection{Phonon side bands}

Intense transitions for $\mathrm{Eu}^{3+}, \mathrm{Gd}^{3+}$ and $\mathrm{Tm}^{3+}$ are accompanied by phonon side bands. This was already shown in chapter 5.1 .3 for $\mathrm{AlN}: \mathrm{Eu}^{3+}$ and these side bands are a severe problem as regards the crystal field analysis in the case of $\mathrm{AlN}: \mathrm{Tm}^{3+}$, which was discussed in the last chapters. Based on the CL spectra for Tm ${ }^{3+}$ in AlN, side bands with energies of $\sim 590 \mathrm{~cm}^{-1}$ can be identified although their exact energy is difficult to determine because they are often overlapping zero-phonon transitions. The phonon side bands are best seen in the case of $\mathrm{Gd}^{3+}$ in AlN (see figure 5.3, where at the lower energy side of the dominant ${ }^{6} P_{7 / 2} \rightarrow{ }^{8} S_{7 / 2}$ transition no additional luminescence is expected and where at the higher energy side the next zero-phonon transition ${ }^{6} P_{5 / 2} \rightarrow{ }^{8} S_{7 / 2}$, is observed at a distance of about $600 \mathrm{~cm}^{-1}$ above the ${ }^{6} P_{7 / 2} \rightarrow{ }^{8} S_{7 / 2}$ transition. Individual phonon side bands were identified and summarized in table 7.7. Two main phonon energies of $588(1) \mathrm{cm}^{-1}(72.9 \mathrm{meV})$ and $100(1) \mathrm{cm}^{-1}(12.4 \mathrm{meV})$ are extracted from experimental data. The Anti-Stokes-side band at around $306.6 \mathrm{~nm}$ is probably masking the transition from ${ }^{6} P_{3 / 2}$ to the ground state. The differences between the side bands corresponding to a certain multiplet and the same phonon are a result of the difficulty in fitting the more or less broadened replica. 
7.6. PHONON SIDE BANDS

\begin{tabular}{ccccc}
\hline \hline$\lambda^{\mathrm{a}}$ & $\mathrm{E}^{\mathrm{b}}$ & No. $^{\mathrm{c}}$ & $\Delta E^{\mathrm{d}}$ & Transition \\
\hline 312.91 & 31949 & 34 & 101 & \\
313.14 & 31926 & 35 & 103 & ${ }^{6} P_{5 / 2} \rightarrow^{8} S_{7 / 2}$ \\
313.21 & 31918 & 36 & 100 & \\
& & & & \\
316.94 & 31342 & 37 & -98 & \\
317.14 & 31324 & 38 & -100 & \\
323.77 & 30877 & 37 & 100 & \\
324.00 & 30856 & 38 & 103 & \\
324.20 & 30837 & 40 & 98 & \\
318.73 & 31366 & 37 & 588 & \\
318.97 & 31342 & 38 & 589 & \\
319.15 & 31324 & 40 & 586 & \\
\hline \hline
\end{tabular}

${ }^{a}$ Experimental wavelength in nanometers, recorded at $300 \mathrm{~K}$.

${ }^{\mathrm{b}}$ Corresponding vacuum energies.

${ }^{c}$ Corresponding zero phonon lines (cf. figure 7.4).

${ }^{\mathrm{d}}$ Positive values correspond to Stokes, negative values to Anti-Stokes luminescence.

Table 7.7: Experimental phonon sidebands for $\mathrm{Gd}^{3+}$ in AlN. Only those side bands are listed which are sufficiently sharp and not masked by other transitions.

Such phonon side bands which represent local modes are well known for lanthanide doped semiconductors and are in more detail reported for $\mathrm{GaN}: \mathrm{Pr}^{3+}$ [30], see also $\mathrm{Si}^{3} \mathrm{Er}^{3+}$ [13]. For the case of $\mathrm{AlN}: \mathrm{Tb}^{3+}$ and $\mathrm{AlN}: \mathrm{Eu}^{3+}$ reported in the literature [43] the authors mention that similar local phonon modes were observed experimentally. A simplified treatment of such local phonon modes was given by Thomas and Hopfield [305] for nitrogen substituents in the semiconductor $\mathrm{GaP}$; they derived the following relationship between the undisturbed optical lattice vibration frequency $\omega_{0}$ and the local mode frequency $\omega_{l o c}$ due to an impurity, which, applied for the case of $\mathrm{Ln}^{3+}$ in AlN reads:

$$
\omega_{0}=\omega_{l o c} \sqrt{\frac{\frac{1}{M_{A l}}+\frac{1}{M_{N}}}{\frac{1}{M_{L n}}+\frac{1}{\alpha M_{N}}}},
$$

where $M_{A l}, M_{N}$, and $M_{L n}$ are the masses of the atoms and $\alpha$ is an empirical constant describing the strength of the localization. The underlying model of a linear chain, made out of atoms of two different masses, implies that the force constants between aluminum and nitrogen atoms 
and impurity and nitrogen atoms are the same..$^{3}$

Unfortunately, $\alpha$ is unknown, but was determined by Lozykowski et al. for $\mathrm{Pr}^{3+}$ in $\mathrm{GaN}$ to $\alpha=$ 1. Taking the corresponding masses for ${ }^{27} \mathrm{Al},{ }^{14} \mathrm{~N}$, and ${ }^{158} \mathrm{Gd}$, and $\alpha=1$, the undisturbed optical phonon mode energy corresponding to the experimental energy of $588 \mathrm{~cm}^{-1}$ is calculated to $694 \mathrm{~cm}^{-1}$. This value is relatively close to the energy reported for the $E_{1}$ (TO) phonon in AlN: 670-677 $\mathrm{cm}^{-1}$ [240]. Of course one needs to determine $\alpha$ separately in order to prove the assumption $\alpha=1$ for the case of $\mathrm{AlN}: \mathrm{Ln}^{3+}$. An open question still remains concerning the origin of the phonon side band with the lower energy of $100 \mathrm{~cm}^{-1}$. This mode is also likely to be a local phonon mode but, following the approach used above, it must be strongly localized since $\alpha \approx 9.5$ is required to reproduce a local mode with an energy of $100 \mathrm{~cm}^{-1}$, corresponding to the $E_{1}(\mathrm{TO})$ phonon in the AlN lattice.

\subsection{Summary and discussion}

In summary, a detailed crystal field splitting analysis of $\mathrm{Tm}^{3+}\left(4 f^{12}\right)$ and $\mathrm{Gd}^{3+}\left(4 f^{7}\right)$ was reported in the last chapters. Modeling of the observed crystal field splitting supports the result of the lattice location studies that both $\mathrm{Tm}^{3+}$ and $\mathrm{Gd}^{3+}$ ions substitutionally replace aluminum atoms in lattice sites with $\mathrm{C}_{3 v}$ point group symmetry in the hexagonal AlN. All CL spectra were associated with the ions on a single site. Due to the results of the calculations, the most important experimentally observed transitions could be assigned. In addition, the branching ratios were discussed, and it was shown that the $\Omega_{2}$ intensity parameter determines the spectra for the case of $\mathrm{Tm}^{3+}\left(4 f^{12}\right)$ in AlN. Phonon sidebands, which were observed for $\mathrm{Eu}^{3+}, \mathrm{Gd}^{3+}$, and $\mathrm{Tm}^{3+}$, were assigned to local phonon modes. For the case of $\mathrm{Gd}^{3+}$ in AlN local phonon modes with energies of $100 \mathrm{~cm}^{-1}$ and $588 \mathrm{~cm}^{-1}$ were experimentally observed. The local mode with an energy of $588 \mathrm{~cm}^{-1}$ is thus likely the corresponding local mode belonging to the $E_{1}$ (TO) phonon in the AlN lattice.

However, a crystal field analysis covers only a part of possible investigations. Nothing was said about the free ion parameters of $\mathrm{Tm}^{3+}$ and $\mathrm{Gd}^{3+}$ in AlN. These may be determined, independently from the crystal field analysis, from the positions of the barycenters of the $L S J$ multiplets. As there are up to 19 free ion parameters in the case of $\mathrm{Gd}^{3+}$, only some of them would be derivable from the spectroscopic studies shown here, because the number of experimentally confirmed multiplet manifolds is lower than 19. It is important to note that, for the calculation of transition probabilities, it is in good approximation sufficient to work with the

\footnotetext{
${ }^{3}$ Exactly this assumption is used to estimate the vibration amplitude of emitter atoms in the scope of emission channeling calculations (see equation 3.60 .
} 


\subsection{SUMMARY AND DISCUSSION}

mean free ion parameters listed in table A.1. With respect to the fact that many of the operators associated with the free ion parameters are not orthogonal to each other and fitting may therefore become a difficult task, working with mean free ion parameters is preferable. Especially for $\mathrm{Gd}^{3+}$ it is difficult to determine the Slater parameters $F^{2}, F^{4}$, and $F^{6}$ as well as Tree's parameters $\alpha, \beta$ and $\gamma$, because the corresponding reduced matrix elements for the experimentally observed transitions are small. The only exception in this case is the spin-orbit parameter $\zeta$.

With regard to the position of the $L S J$ multiplets, sometimes a remarkable red-shift was observed. This can be clearly seen for $\mathrm{Tm}^{3+}$ in AlN, when looking at the ${ }^{1} D_{2} \rightarrow{ }^{3} H_{6}$ transition, as well as for $\mathrm{Gd}^{3+}$ in AlN when looking at the ${ }^{6} P_{7 / 2} \rightarrow{ }^{8} S_{7 / 2}$ transition. The initial Stark levels were found at energies a few hundred wavenumbers lower compared to the predictions of the mean free ion parameters. This behavior is called nephelauxetic effect and may be interpreted in terms of an expansion of the radial integrals of the $4 f$ wavefunctions. Such a behavior is observed in case of a pronounced covalent bonding between a lanthanide ion and surrounding ligands. It manifests itself in a decrease in the rank-2 Slater parameter $F^{2}$, because an expansion of the $4 f$ radial wavefunction will decrease the Coulomb repulsion between $4 f$ electrons. For $\mathrm{Tm}^{3+}$ and $\mathrm{Eu}^{3+}$ in $\mathrm{c}-\mathrm{BN}$ such a red shift was also observed, but its magnitude is not clearly visible due to the obviously large crystal field splitting. Because $\mathrm{c}-\mathrm{BN}$ is even more covalent than AlN, the nephelauxetic effect should be more pronounced for $\mathrm{c}-\mathrm{BN}$. 


\section{Summary and concluding remarks}

In this work lattice location studies together with cathodoluminescence investigations of europium, thulium, and gadolinium implanted into the wide band gap semiconductors AlN, c$\mathrm{BN}, 3 \mathrm{C}-\mathrm{SiC}, 4 \mathrm{H}-\mathrm{SiC}, 6 \mathrm{H}-\mathrm{SiC}$, as well as diamond, were performed. These investigations were complemented by a crystal field analysis for thulium and gadolinium in AlN. Prior to these investigations, experimental setups were assembled with the focus on the development of important extensions such as facilities for time-resolved cathodoluminescence measurements.

\section{Lattice location studies}

Conversion electron emission channeling studies of ${ }^{169} \mathrm{Yb}$ ions implanted into AlN, revealed a substitutional $\mathrm{Al}$ (cationic) site for the implanted ions. Due to the fact that lanthanides are very similar with respect to their chemical properties, the same lattice site was assumed for other lanthanide ions such as thulium or gadolinium.

For $\mathrm{c}-\mathrm{BN}$, conversion electron emission channeling studies of implanted ${ }^{139} \mathrm{Ce}$ ions suggest that the implanted ions are either located on near-subsitutional boron sites or occupy different sites in the $\mathrm{c}-\mathrm{BN}$ host. The lattice location studies, performed after annealing at $1173 \mathrm{~K}$ revealed only a small fraction of the implanted ions located on these sites. This suggests that much higher annealing temperatures are necessary to anneal the defects in the c-BN host after ion implantation. Further investigations are necessary in order to unambiguously identify the lattice sites of lanthanides in $\mathrm{c}-\mathrm{BN}$.

For the lanthanides $\mathrm{Yb}$ and $\mathrm{Tm}$, implanted into $3 \mathrm{C}-, 4 \mathrm{H}-$ and $6 \mathrm{H}-\mathrm{SiC}$ a near substitutional silicon sites was identified. For $\mathrm{Tm}$ in $3 \mathrm{C}-\mathrm{SiC}$ a fraction of $46(9) \%$ of the implanted ions was found to be located on substitutional silicon sites. For Tm in $4 \mathrm{H}-\mathrm{SiC}$, a fraction of 58(5) \% of the implanted ions was found to be located on substitutional silicon sites upon implantation. For $\mathrm{Yb}$ in $6 \mathrm{H}-\mathrm{SiC}$, more than $50 \%$ of the implanted ions were found on near-substitutional silicon sites. Annealing the samples led to an increase in the fraction of the implanted ions on substitutional lattice sites in all three $\mathrm{SiC}$ polytypes. The observed behavior is in contrast to the behavior of rare earth impurities in III-V semiconductors like GaN and AIN.

In the case of a single crystal diamond, radioactive ${ }^{141} \mathrm{Ce}$ and ${ }^{149} \mathrm{Gd}$ probe isotopes were used for emission channeling investigations for the first time. After room temperature implantation and annealing at $1200 \mathrm{~K}, 40-50 \%$ of the implanted lanthanide atoms occupied sites displaced from substitutional sites by up to $0.45(10) \AA$ along the $<111>$ directions, or with a mean projected displacement of $\mathrm{d} \approx 0.26 \AA$. The situation was therefore found to be different to that observed for lanthanide impurities in Si [186, 213], where up to $90 \%$ of the implanted probes were observed 
to take up near-tetrahedral-interstitial sites.

An important contribution to the emission channeling method was given due to the use of new isotopes for the first time, namely ${ }^{139} \mathrm{Ce},{ }^{141} \mathrm{Ce}$, and ${ }^{149} \mathrm{Gd}$. With the increasing importance of the emission channeling method, as the only method currently available for direct lattice location studies of implanted impurities, the opening of new elements is of prior importance. For a crystal field analysis the position of the implanted ions with respect to the unit cell is one of the most requested input parameters. Furthermore important numerical extensions were realized, which allow to calculate theoretical emission channeling spectra, even for complex unit cells.

\section{Cathodoluminescence}

Gadolinium implanted AlN, annealed at $1373 \mathrm{~K}$ under vacuum conditions, revealed to be a very promising candidate for the implementation of ultra violet light emitters. The dominant luminescence related to $\mathrm{Gd}^{3+}$ was monitored at $318 \mathrm{~nm}$. It was attributed to the ${ }^{6} P_{7 / 2} \rightarrow{ }^{8} S_{7 / 2}$ intra- $4 f$ electron transition of $\mathrm{Gd}^{3+}$ between the first excited state and the ground state. Lifetime measurements of the ${ }^{6} P_{7 / 2}$ level showed a slightly decreasing lifetime with increasing temperature, reaching $0.69 \mathrm{~ms}$ at $300 \mathrm{~K}$. This decrease in lifetime was compared to the increase in integral intensity of the ${ }^{6} P_{7 / 2} \rightarrow{ }^{8} S_{7 / 2}$ transition with increasing temperature. It was shown that the increasing intensity must be attributed to the excitation mechanism.

Europium implanted AIN, annealed at $1373 \mathrm{~K}$ under vacuum conditions, showed characteristic luminescence due to intra- $4 f$ electron transitions of $\mathrm{Eu}^{3+}$ at around $620 \mathrm{~nm}$, making this system an interesting candidate for red light emitters. Is was exemplarily shown for the case of the ${ }^{5} D_{0} \rightarrow{ }^{7} F_{1}$ and ${ }^{5} D_{1} \rightarrow{ }^{7} F_{1}$ transitions, how final multiplets of transitions in luminescence spectra are identified. Lifetime measurements monitoring the dominant ${ }^{5} D_{0} \rightarrow{ }^{7} F_{2}$ transition showed an almost constant lifetime around $360 \mu$ s over the temperature range $12 \mathrm{~K}$ to $300 \mathrm{~K}$.

Thulium implanted AlN, annealed at $1273 \mathrm{~K}$ under vacuum conditions, showed a dominant blue light emission at around $465 \mathrm{~nm}$. Based on lifetime measurements and the crystal field analysis, the dominating blue emission at $300 \mathrm{~K}$ was attributed to the ${ }^{1} D_{2} \rightarrow{ }^{3} F_{4}$ intra- $4 f$ electron transition of $\mathrm{Tm}^{3+}$. The survey spectra, recorded at $12 \mathrm{~K}$ and $300 \mathrm{~K}$, revealed remarkable differences. While at $12 \mathrm{~K} \mathrm{Tm}^{3+}$-related luminescence was dominated by transitions starting from the ${ }^{1} I_{6}$ level, the luminescence at $300 \mathrm{~K}$ was dominated by transitions starting from the ${ }^{1} D_{2}$ level. Lifetime measurements of both initial levels suggested that with increasing temperature the ${ }^{1} I_{6}$ level is depopulated non-radiatively to the ${ }^{1} D_{2}$ level, there efficiently populating the latter one.

Europium-implanted c-BN, annealed under high temperature and high pressure conditions, 
clearly showed luminescence due to the ${ }^{5} D_{0} \rightarrow{ }^{7} F_{2}$ intra- $4 f$ electron transition of $\mathrm{Eu}^{3+}$. Additionally, in poly crystal c-BN specimens, luminescence arising from $4 f^{N-1} 5 d \rightarrow 4 f^{N}$ electron transitions of $\mathrm{Eu}^{3+}$ was visible. Luminescence involving the upper initial ${ }^{5} D_{1}$ level were found to be absent. This was attributed to high optical phonon frequencies and the pronounced covalency of the c-BN host. Thulium-implanted c-BN, annealed under high temperature and high pressure conditions, showed only moderate luminescence related to intra- $4 f$ electron transitions of $\mathrm{Tm}^{3+}$. With increasing temperature a decrease of the integral intensity of dominant $\mathrm{Tm}^{3+}$-related luminescence can be seen. Using $\mathrm{c}-\mathrm{BN}$ as a host for the implementation of lanthanide doped light emitters is therefore promising, although the experimental results have clearly shown that $\mathrm{c}-\mathrm{BN}$ is much more difficult to handle than AlN. An important point will be whether $\mathrm{c}-\mathrm{BN}$ can be doped with lanthanides during growth. Such experiments are being prepared.

Cathodoluminescence of thulium implanted $6 \mathrm{H}-\mathrm{SiC}$ specimens, annealed under vacuum conditions and a temperature of $1473 \mathrm{~K}$, revealed room-temperature infrared luminescence around $805 \mathrm{~nm}$. This luminescence was related to radiative ${ }^{3} H_{4} \rightarrow{ }^{3} H_{6}$ intra- $4 \mathrm{f}$ electron transitions of $\mathrm{Tm}^{3+}$. Transitions starting from the ${ }^{1} G_{4}$ level are absent at higher temperatures.

\section{Thin films}

The doping of MSIBD grown ta-C and $\mathrm{c}-\mathrm{BN}$ films with europium was investigated. The films were doped with Eu during growth by co-deposition. The application of different methods, such as photoelectron, cathodoluminescence and conversion electron Mössbauer spectroscopy, for the investigation of such films was shown. Successful incorporation of europium in the charge state $3+$ and activation of $\mathrm{Eu}^{3+}$ intra-4f luminescence were achieved for co-deposited c-BN:Eu films. For the ta-C:Eu film an accumulation of Eu at the surface was observed and an incorporation of Eu into the ta-C matrix seemed to be difficult. In contrast to the luminescence behavior of the europium-implanted bulk c-BN species, the luminescence related to intra- $4 \mathrm{f}$ electron transitions of $\mathrm{Eu}^{3+}$ in the c-BN thin film was found to be much weaker. Possible explanation are provided by the low solubility of europium in $\mathrm{c}-\mathrm{BN}$ and carrier relaxation at c-BN grain boundaries and the $\mathrm{Si} / \mathrm{t}-\mathrm{BN} / \mathrm{c}-\mathrm{BN}$ interface.

\section{Crystal field analysis}

A detailed crystal field splitting analysis of $\mathrm{Tm}^{3+}\left(4 f^{12}\right)$ and $\mathrm{Gd}^{3+}\left(4 f^{7}\right)$ in $2 \mathrm{H}$-AlN was reported in this work. This was the first time that a detailed crystal field analysis for these ions in a semiconductor host was performed, and the first time that a semiconductor with a band gap larger than $4 \mathrm{eV}$ was used for such studies. Modeling of the observed crystal field splitting supports the lattice location studies; both $\mathrm{Tm}^{3+}$ and $\mathrm{Gd}^{3+}$ ions substitutionally replace aluminum atoms 
in lattice sites with $\mathrm{C}_{3 v}$ point group symmetry in $2 \mathrm{H}-\mathrm{AlN}$. All CL spectra were associated with the ions in a single site. From the lattice sum calculations the most important experimental transitions could be assigned. In addition, the branching ratios were discussed, and it was shown that the $\Omega_{2}$ intensity parameter seems to determine the spectra for $\mathrm{Tm}^{3+}\left(4 f^{12}\right)$ in AlN. Phonon sidebands, which were observed for $\mathrm{Eu}^{3+}, \mathrm{Gd}^{3+}$, and $\mathrm{Tm}^{3+}$, were assigned to local phonon modes. For $\mathrm{Gd}^{3+}$ in AlN local phonon modes with energies of $100 \mathrm{~cm}^{-1}$ and $588 \mathrm{~cm}^{-1}$ were experimentally observed. The local mode with an energy of $588 \mathrm{~cm}^{-1}$ is likely the corresponding local mode belonging to the $E_{1}(\mathrm{TO})$ phonon in the AlN lattice.

\section{The Future}

Based on the experimental results presented in this work, numerous further investigations are conceivable. An important question that could not be answered due to the use of cathodoluminescence as the excitation method, is the nature and path of the excitation. It was already pointed out in the very beginning, that such investigations are best performed with $p n$-junctions, operated in forward as well as reverse biased mode. Because such devices are difficult to fabricate, an alternative first step can be seen in the implementation of thin film electroluminescence devices. With reference to the observed behavior, these should be implementable for $\mathrm{Tm}^{3+}$ in AlN, $6 \mathrm{H}-\mathrm{SiC}$ and c-BN, $\mathrm{Eu}^{3+}$ in AlN and c-BN, and $\mathrm{Gd}^{3+}$ in AlN. In the next steps the fabrication of $p n$-junctions and heterostructures should be addressed. The most important outcome of such studies are the excitation cross sections. Additionally the use of laser light for above band gap excitation is preferable, because the excitation path will be better defined compared to cathodoluminescence, where besides excitation through excitons or the lanthanide trap, impact excitation and impact ionization may occur.

An important aspect of lanthanide doped wide band gap semiconductors is the possibility of designing light emitting devices emitting selectively at different wavelengths. The behavior observed for $\mathrm{Tm}^{3+}$ in AlN suggests that the branching ratios may be altered by changing the ligand field around the lanthanide ions. How this may be realized is an open question. The use of hydrogen is only one possible approach. The reported temperature-dependent luminescence of $\mathrm{Tm}^{3+}$ in AlN may make this system interesting for heat sensors.

Another interesting field of research using the above substrates might be found when reducing the dimensions of the films to sizes where quantum effects play a role. Using lanthanides as luminescence probes for such quantum structures is a very promising development currently taking place. A typical experimental result is the refractive index of the quantum structure, which alters with the size of the structure and has direct influence on the Einstein coefficient for spontaneous emission. Moreover, because lifetimes of energy carriers in quantum structures may be longer than in the bulk material, the lanthanide-related luminescence can be increased 
significantly.

Radioactive isotopes play an important role in the investigations of lanthanide doped semiconductors. Unfortunately, such isotopes are more and more difficult to source. Moreover, at present, methods that are usually applied in hyperfine investigations, such as Mössbauer spectroscopy or perturbed $\gamma \gamma$-angular correlation, are still experimentally not sufficiently developed for the use of radioactive lanthanide isotopes. This will hopefully be overcome in the future. Fortunately, experimental setups for emission channeling investigations were constantly improved during the last decade.

The use of radioactive lanthanides in luminescence investigations is generally very attractive. It allows, in principle, to probe different lanthanides in the same sample, and may therefore be interesting for crystal field investigations as well. Among the lanthanides, promethium is the only element without a stable isotope. For this reason the spectroscopic studies on $\mathrm{Pm}^{3+}$ are very rare, and significant contributions to existing data may come from $\mathrm{Pm}^{3+}$-implanted wide band gap semiconductors. Currently, cathodoluminescence as well as photoluminescence investigations for both $\mathrm{GaN}$ and $\mathrm{AlN}$, implanted with radioactice Promethium atoms, are being prepared.

The crystal field analyses as well as considerations regarding the branching ratios have shown the large potential of using wide band gap semiconductors in the analysis of the luminescence spectra of triply ionized lanthanides. It is worth pointing out that many insulator crystal hosts show strong lattice absorption above $35,000 \mathrm{~cm}^{-1}$. Significant contributions to experimental data as well as theoretical calculations are therefore expected in the future in the case of lanthanide implanted semiconductors, complementing the development initiated by lanthanide doped inorganic insulators during the last decades. The semiconductor aluminum nitride has proven to be currently the most interesting candidate for such studies, with regard to the energy level structure of e.g. $\mathrm{Gd}^{3+}$ and $\mathrm{Tm}^{3+}$ even beating gallium nitride, but other attractive hosts such as c-BN, h-BN, $\mathrm{ZnS}$ and $\mathrm{ZnO}$, or ternary compounds such as $\mathrm{Al}_{x} \mathrm{Ga}_{1-x} \mathrm{~N}$ will also play an important role. 


\section{A Appendix}

\section{A.1 Mean free ion parameters for $\mathbf{E u}^{3+}, \mathbf{G d}^{3+}$ and $\mathbf{T m}^{3+}$}

\begin{tabular}{|c|c|c|c|c|c|}
\hline & Interaction & Parameter & $\mathrm{Eu}^{3+}\left(f^{6}\right)$ & $\mathrm{Gd}^{3+}\left(f^{7}\right)$ & $\mathrm{Tm}^{3+}\left(f^{12}\right)$ \\
\hline $\begin{array}{l}\text { spherically } \\
\text { symmetric }\end{array}$ & all & $E_{A V E}$ & 63736 & 87446 & 17677 \\
\hline \multirow{6}{*}{$\begin{array}{l}\text { angular } \\
\text { dependent }\end{array}$} & Coulomb & $\begin{array}{l}F^{2} \\
F^{4} \\
F^{6}\end{array}$ & $\begin{array}{l}82786 \\
60517 \\
42644\end{array}$ & $\begin{array}{l}85300 \\
59401 \\
44731\end{array}$ & $\begin{array}{c}101381 \\
70230 \\
51827\end{array}$ \\
\hline & spin-orbit coupling & $\zeta$ & 1332 & 1504 & 2644 \\
\hline & $\begin{array}{l}\text { two-particle } \\
\text { interaction }\end{array}$ & $\begin{array}{l}\alpha \\
\beta \\
\gamma\end{array}$ & $\begin{array}{l}19.80 \\
-617 \\
1460\end{array}$ & $\begin{array}{l}18.95 \\
-620 \\
1658\end{array}$ & $\begin{array}{l}17.25 \\
-665 \\
1936\end{array}$ \\
\hline & $\begin{array}{l}\text { three-particle } \\
\text { interaction }\end{array}$ & $\begin{array}{l}T^{2} \\
T^{3} \\
T^{4} \\
T^{6} \\
T^{7} \\
T^{8}\end{array}$ & $\begin{array}{c}370 \\
43 \\
51 \\
-330 \\
380 \\
370\end{array}$ & $\begin{array}{c}308 \\
40 \\
40 \\
-298 \\
338 \\
335\end{array}$ & $\begin{array}{l}- \\
- \\
- \\
- \\
- \\
-\end{array}$ \\
\hline & $\begin{array}{l}\text { spin-spin and } \\
\text { spin-other-orbit }\end{array}$ & $\begin{array}{l}M^{0} \\
M^{2} \\
M^{4}\end{array}$ & $\begin{array}{l}2.38 \\
1.33 \\
0.90\end{array}$ & $\begin{array}{l}2.99 \\
1.67 \\
1.44\end{array}$ & $\begin{array}{l}4.93 \\
2.76 \\
1.87\end{array}$ \\
\hline & spin-orbit & $\begin{array}{l}P^{2} \\
P^{4} \\
P^{6}\end{array}$ & $\begin{array}{l}303 \\
227 \\
152\end{array}$ & $\begin{array}{l}542 \\
407 \\
271\end{array}$ & $\begin{array}{l}730 \\
548 \\
365\end{array}$ \\
\hline
\end{tabular}

Table A.1: Mean free ion parameters for the trivalent lanthanide ions $\mathrm{Eu}^{3+}, \mathrm{Gd}^{3+}$ and $\mathrm{Tm}^{3+}$ and their corresponding interaction, taken from ref. [106]. All values are in $\mathrm{cm}^{-1}$. 


\section{A.2 Substrates}

In table A.2 an overview of the substrates used in this work is given. Some of the substrates are commercially available (AlN,3C-SiC,4H-SiC,6H-SiC) or were kindly supplied (AlN, diamond, $\mathrm{c}-\mathrm{BN})$. A further discussion of the properties of the substrates is given below. In table

\begin{tabular}{cccc}
\hline \hline Substrate & Orientation & Comments & Supplier \\
\hline 2H-AlN & $(0001)$ & $>1 \mu$ m epit. film on 6H-SiC & Raleigh \\
& & $200 \mathrm{~nm}$ epit. film on 6H-SiC & University Wafers \\
c-BN & $<100>$ & bulk & T. Taniguchi \\
& $<111>$ & bulk & T. Taniguchi \\
& polycrystalline & bulk & T. Taniguchi \\
$6 \mathrm{H}-\mathrm{SiC}$ & $(0001)$ & $>1 \mu$ m epit. on 6H-SiC, n-type & Cree Industries \\
& & n-tpye & SiCrystal \\
$4 \mathrm{H}-\mathrm{SiC}$ & $(0001)$ & semi-insulating, bulk & Cree Industries \\
$3 \mathrm{C}-\mathrm{SiC}$ & $<100>$ & semi-insulating, bulk & Cree Industries \\
Diamond & $<100>$ & type IIa, bulk & de Beers \\
& $<111>$ & type IIa, bulk & de Beers \\
\hline
\end{tabular}

Table A.2: Overview of the substrates used in this work.

A.3 the physical properties of the different materials are summarized; as long as these properties are needed for experiments in this work. The unit cells of $\mathrm{AlN}$ and $3 \mathrm{C}-\mathrm{SiC}, 4 \mathrm{H}-\mathrm{SiC}$ and $6 \mathrm{H}-\mathrm{SiC}$ are shown in figure $\mathrm{A} .1$; the unit cells of diamond and $\mathrm{c}-\mathrm{BN}$ are not shown separately as they possess, like the $3 \mathrm{C}-\mathrm{SiC}$ polytpye, $\mathrm{ZnCl}$ structure. 


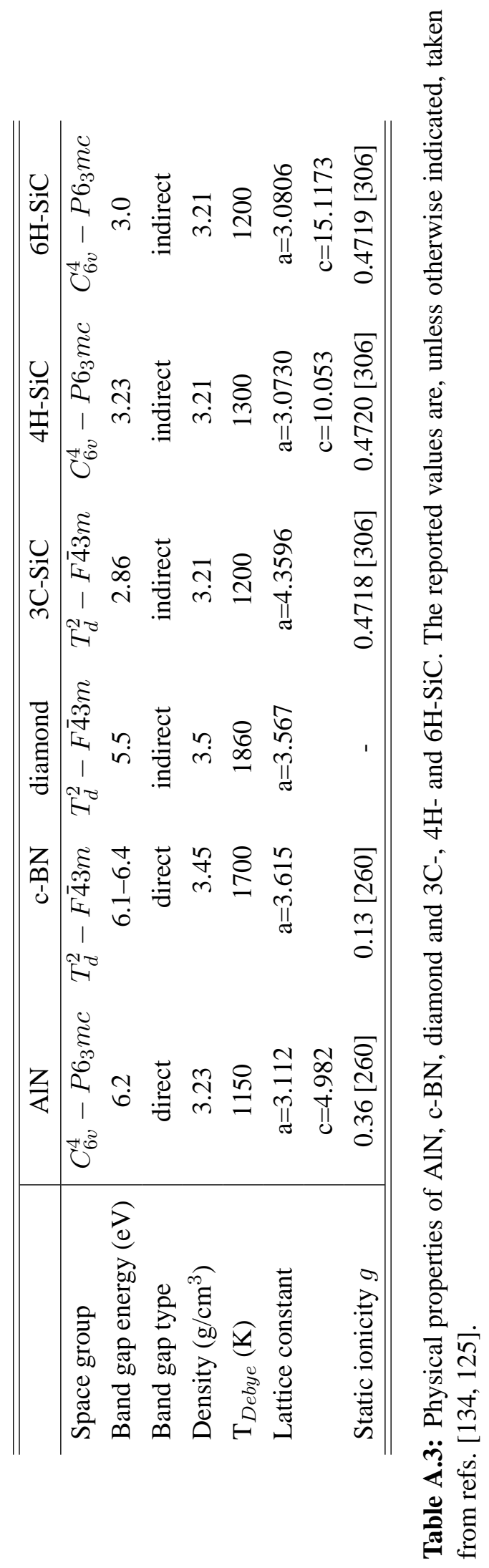



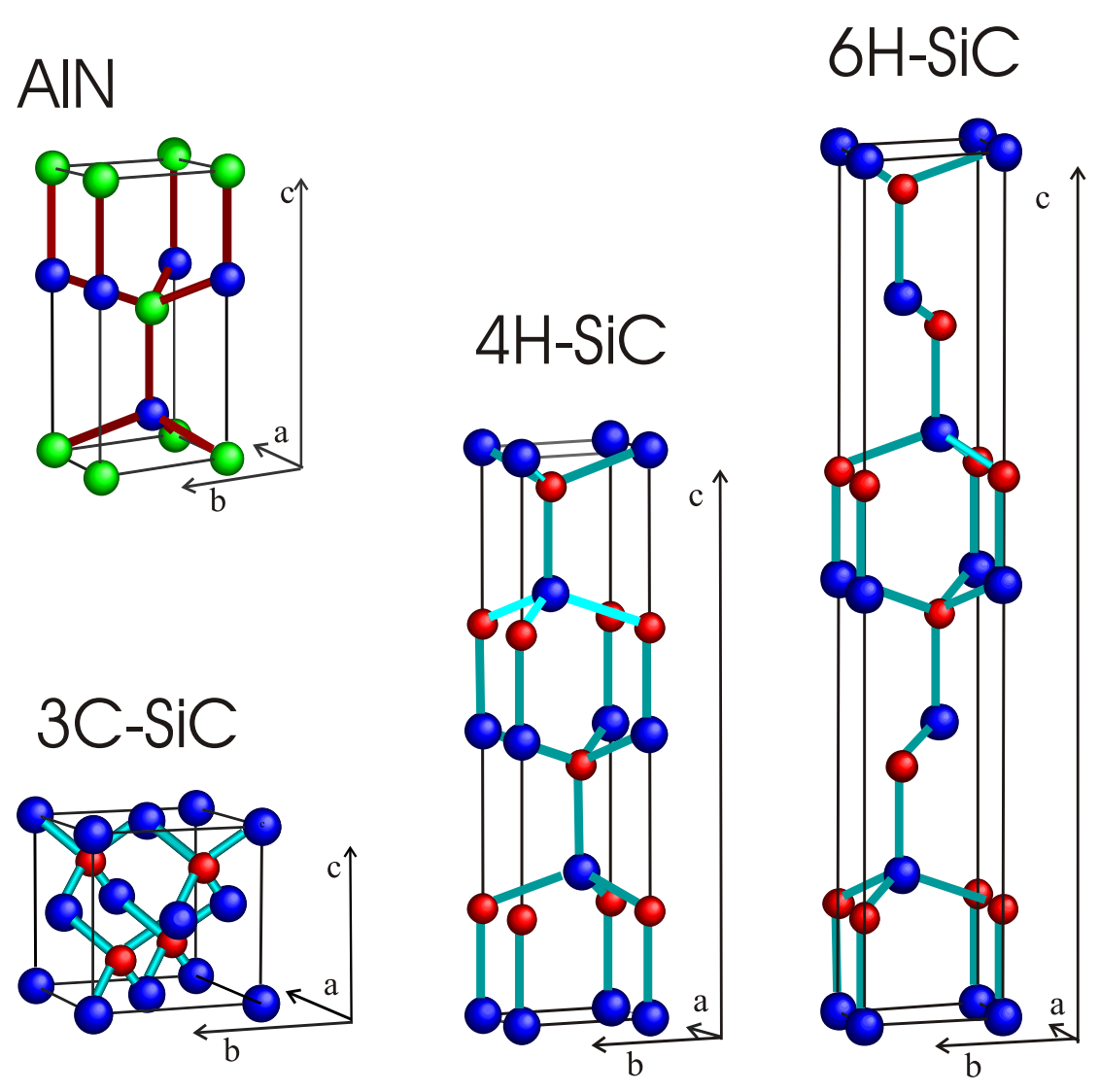

Figure A.1: Unit cells of AlN, 3C-SiC, $4 \mathrm{H}-\mathrm{SiC}$ and $6 \mathrm{H}-\mathrm{SiC}$. The unit cells of c-BN and diamond are not listed separately; their structure is similar to that of the $3 \mathrm{C}-\mathrm{SiC}$ unit cell. 


\section{List of Figures}

$2.1 \mathrm{Ln}^{3+}$ excitation mechanisms. . . . . . . . . . . . . . 5

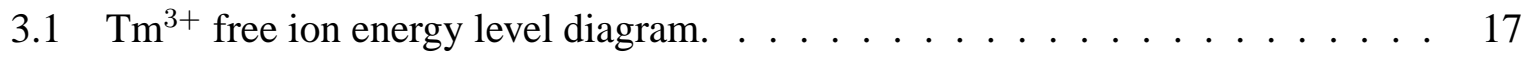

3.2 The Dieke diagram. . . . . . . . . . . . . . . . . . . . . . . . . . . . . . . . 19

3.3 Lanthanide ion in w-AlN unit cell. . . . . . . . . . . . . . . . . 23

$3.4 \quad$ Wavelength dependent correction factor $\chi \bar{\nu}_{0}^{3}$ for an oscillator in a dielectric medium in the case of emission, shown for $6 \mathrm{H}-\mathrm{SiC}$ and $2 \mathrm{H}-\mathrm{GaN}.] \ldots \ldots .31$

$3.5 \quad$ Band gap vs. max. phonon frequency of selected semiconductors. . . . . . . . 34

3.6 Fractional thermal population. . . . . . . . . . . . . . . 35

$3.7 \quad$ Excitation and deexcitation spectrum. . . . . . . . . . . . 36

3.8 Selected squared reduced matrix elements for $\mathrm{Tm}^{3+}, \mathrm{Eu}^{3+}$ and $\mathrm{Gd}^{3+} \ldots \ldots 43$

3.9 Electron energy spectra and decay and level-schemes of lanthanide isotopes

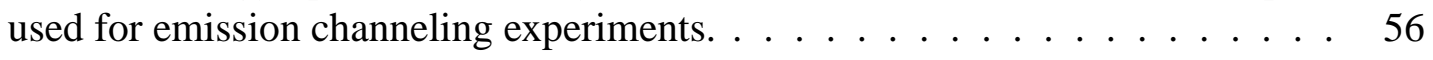

3.10 Mössbauer spectrum of ${ }^{149} \mathrm{Sm}$ in GaN. . . . . . . . . . . . . . . . . 61

3.11 Low-energy $\gamma$-spectrum of ${ }^{149} \mathrm{Sm}$ and ${ }^{151} \mathrm{Eu} . \ldots \ldots \ldots \ldots$. . . . . . . 61

4.1 Schematic diagram of the luminescence setup. . . . . . . . . . . . 63

4.2 Diagram of the electronic part of the CL/PL setup. . . . . . . . . . . 65

$4.3{ }^{4} I_{13 / 2} \rightarrow{ }^{4} I_{15 / 2}$ transition of $\mathrm{Er}^{3+}$ in Si. $\ldots \ldots \ldots \ldots \ldots \ldots$

4.4 Circuit diagram of the pulse unit. . . . . . . . . . . . . . . . . . . 69

4.5 Time resolution of the CL unit. . . . . . . . . . . . . . . . 70

4.6 Principle of an emission channeling setup. . . . . . . . . . . . 73

4.7 Deetee detector wiring. . . . . . . . . . . . . . . . . . . . 75

4.8 CEMS detector technical drawings. $\ldots \ldots \ldots \ldots \ldots$

$5.1 \quad$ Emission channeling spectra of Tm-implanted AlN. . . . . . . . . . . . . 81

5.2 Cathodoluminescence spectrum of Gd implanted AlN. . . . . . . . . . . 83

5.3 The ${ }^{6} P_{7 / 2} \rightarrow{ }^{8} S_{7 / 2}$ transition and phonon sidebands. . . . . . . . . . . 84

5.4 Decay of the ${ }^{6} P_{7 / 2}$ level of $\mathrm{Gd}^{3+}$ in AlN. $\ldots \ldots \ldots \ldots \ldots . \ldots . \ldots$

5.5 Cathodoluminescence survey scans of $\mathrm{Eu}^{3+}$ in AlN. . . . . . . . . . 86 
5.6 High-resolution spectra of the ${ }^{5} D_{0} \rightarrow{ }^{7} F_{1}$ and ${ }^{5} D_{1} \rightarrow{ }^{7} F_{1}$ transition of $\mathrm{Eu}^{3+}$ in

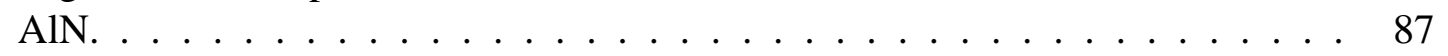

$5.7 \quad$ Luminescence decay of the ${ }^{5} D_{0}$ level of $\mathrm{Eu}^{3+}$ in AlN. . . . . . . . . . . . . 88

$5.8 \quad$ CL spectrum of Tm-implanted AlN. . . . . . . . . . . . . . . . . . . . . 89

5.9 Luminescence decay of the ${ }^{1} I_{6}$ and ${ }^{1} D_{2}$ levels of $\mathrm{Tm}^{3+}$ in AlN. . . . . . . . 90

5.10 Photograph of c-BN single crystals. . . . . . . . . . . . . . . . 93

5.11 Emission channeling spectra of ${ }^{139} \mathrm{Cein} \mathrm{c}-\mathrm{BN} \ldots \ldots \ldots$ \begin{tabular}{|l}
5.12 CL survey spectra of $\mathrm{Eu}^{3+}$-implanted poly- and single crystalline c-BN, mea- \\
sured at $12 \mathrm{~K}$ and $300 \mathrm{~K} . \ldots \ldots \ldots \ldots$
\end{tabular}

5.13 High resolution CL spectrum of the ${ }^{5} D_{0} \rightarrow^{7} F_{J}$ transitions of $\mathrm{Eu}^{3+}$ in c-BN. . . 97

5.14 High resolution and temperature dependent CL spectrum of the ${ }^{5} D_{0} \rightarrow{ }^{7} F_{2}$ transitions of $\mathrm{Eu}^{3+}$ in poly crystal c-BN. $\ldots \ldots \ldots \ldots \ldots . \ldots \ldots$

5.15 Luminescence decay of the ${ }^{5} D_{0}$ level of $\mathrm{Eu}^{3+}$ in c-BN. . . . . . . . . . . . 99

5.16 CL survey spectra of $\mathrm{Tm}^{3+}$-implanted poly- and single crystal c-BN. . . . . . . 100

5.17 Temperature dependent high resolution CL spectra of the ${ }^{1} G_{4} \rightarrow{ }^{3} H_{6}$ and ${ }^{1} D_{2} \rightarrow{ }^{3} F_{4}$ transitions of $\mathrm{Tm}^{3+}$ in c-BN. $\ldots \ldots \ldots \ldots \ldots \ldots$

5.18 Emission channeling spectrum of ${ }^{167} \mathrm{Er}$ in $3 \mathrm{C}-\mathrm{SiC}$, as implanted. . . . . . . . 103

5.19 Emission channeling spectrum of ${ }^{167} \mathrm{Er}$ in $4 \mathrm{H}-\mathrm{SiC}$, as implanted. . . . . . . . . 104

5.20 Emission channeling spectrum of ${ }^{169} \mathrm{Tm}$ in $6 \mathrm{H}-\mathrm{SiC}$ annealed at $1073 \mathrm{~K}$.

5.21 Dependence of the normalized conversion electron yields of ${ }^{167} \mathrm{Tm}$ and ${ }^{169} \mathrm{Yb}$

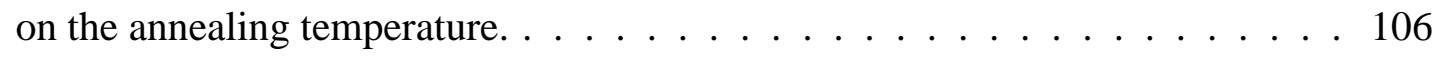

$5.22{ }^{1} G_{4} \rightarrow{ }^{3} H_{6}$ intra-4f electron transition of $\mathrm{Tm}^{3+}$ in $6 \mathrm{H}-\mathrm{SiC} \ldots \ldots \ldots$

5.23 Time resolved CL spectra of the ${ }^{1} G_{4} \rightarrow{ }^{3} H_{6}$ transition of $\mathrm{Tm}^{3+}$ in 6H-SiC. . . . 109

$5.24 \mathrm{Tm}^{3+}$ in SiC CL survey scans at $12 \mathrm{~K}$ and $300 \mathrm{~K} . \ldots \ldots \ldots$

$5.25{ }^{3} H_{4} \rightarrow{ }^{3} H_{6}$ intra-4f electron transition of $\mathrm{Tm}^{3+}$ in $6 \mathrm{H}-\mathrm{SiC} \ldots \ldots \ldots 111$

5.26 Emission channeling spectra of ${ }^{141} \mathrm{Pr}$ in diamond. . . . . . . . . . . . . 113

5.27 Emission channeling spectra of ${ }^{149} \mathrm{Gd}$ in diamond $(<110>$-axis $)$. . . . . . . . 114

5.28 Emission channeling spectra of ${ }^{149} \mathrm{Gd}$ in diamond $(<111>$-axis $)$. . . . . . . . 115

5.29 CL spectra of europium- and thulium- implanted diamond. . . . . . . . . . 116

$6.1 \quad$ Eu 3d core level XPS spectra for ta-C:Eu and c-BN:Eu $\ldots \ldots \ldots$. . . . . . . 120

$6.2 \quad$ FTIR spectra of Eu-doped and undoped c-BN thin films. . . . . . . . . . . 121

6.3 Mössbauer spectra of ${ }^{151} \mathrm{Eu}$ in ta-C and c-BN. . . . . . . . . . . . . . . 122

6.4 CL spectrum of Eu-doped c-BN thin films. . . . . . . . . . . . . . . . . 123 
$7.1 \quad$ High resolution CL spectra of $\mathrm{Tm}^{3+}$ implanted AlN, part I. . . . . . . . . . . 128

$7.2 \quad$ High resolution CL spectra of $\mathrm{Tm}^{3+}$ implanted AlN, part II. $\ldots . . . . . .129$

7.3 Experimental energy level diagram for $\mathrm{Tm}^{3+}$ in AlN. . . . . . . . . . . . . 132

7.4 High resolution spectra of excited levels of $\mathrm{Gd}^{3+}$ in AlN. . . . . . . . . . . 135

7.5 Experimental energy level diagram for $\mathrm{Gd}^{3+}$ in AlN. . . . . . . . . . . 136

7.6 Reduced matrix elements and transition probabilities for transitions from the ${ }^{1} D_{2}$ level of $\mathrm{Tm}^{3+} . \ldots \ldots \ldots \ldots \ldots \ldots \ldots \ldots$

A.1 Unit cells of AlN, 3C-SiC, $4 \mathrm{H}-\mathrm{SiC}$ and $6 \mathrm{H}-\mathrm{SiC} . \quad \ldots \ldots \ldots \ldots$ 
LIST OF FIGURES 


\section{List of Tables}

3.1 Filling of the $4 f$ shells according to Hund's rules. . . . . . . . . . . 11

3.2 Selection rules for induced electric dipole and magnetic dipole transitions. . . . 28

3.3 Lanthanide isotopes suitable for off-line emission channeling investigations. . . 52

3.4 Lanthanide isotopes suitable for Mössbauer spectroscopy studies at room tem-

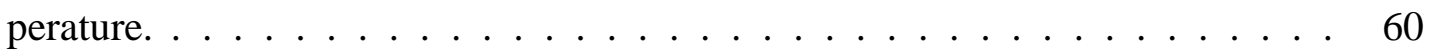

$7.1 \quad$ Experimental transitions of $\mathrm{Tm}^{3+}$ in AlN. . . . . . . . . . . . . . . 131

7.2 Full-rotational group compatibility table for $\mathrm{Tm}^{3+}$ energy levels under symme-

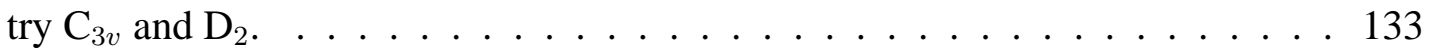

7.3 Energy levels of $\mathrm{Tm}^{3+}\left(4 f^{12}\right)$ in AlN. . . . . . . . . . . . . . . . 134

7.4 Full-rotational group compatibility table for $\mathrm{Gd}^{3+}$ energy levels under symme-

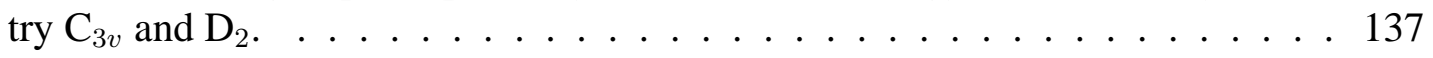

7.5 Energy levels of $\mathrm{Gd}^{3+}\left(4 f^{7}\right)$ in AlN. . . . . . . . . . . . . . . . . . . . . 141

7.6 Experimental and calculated relative intensities for the ${ }^{1} D_{2} \rightarrow^{2 S+1} L_{J}$ transition

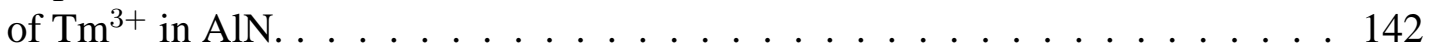

7.7 Experimental phonon sidebands for $\mathrm{Gd}^{3+}$ in AlN. . . . . . . . . . . . . . 145

A.1 Mean free ion parameters for $\mathrm{Eu}^{3+}, \mathrm{Gd}^{3+}$ and $\mathrm{Tm}^{3+} \ldots \ldots \ldots \ldots$

A.2 Overview of the substrates used in this work. . . . . . . . . . . . . . . . 154

A.3 Physical properties of AlN, c-BN, diamond and 3C-, 4H- and 6H-SiC . . . 155 
LIST OF TABLES 


\section{Bibliography}

[1] H. Ennen et al., Appl. Phys. Lett. 43 (1983) 943.

[2] B. Zheng et al., Appl. Phys. Lett. 64 (1994) 2842.

[3] S. Coffa, G. Franzò and F. Priolo, Appl. Phys. Lett. 69 (1996) 2077.

[4] J. Palm et al., Phys. Rev. B 54 (1996) 17603.

[5] C.X. Du et al., Appl. Phys. Lett. 71 (1997) 1023.

[6] A. Reittinger, J. Stimmer and G. Abstreiter, Appl. Phys. Lett. 70 (1997) 2431.

[7] L. Tsybeskov et al., Appl. Phys. Lett. 70 (1997) 1790.

[8] M. Matsuoka and S. Tohno, Appl. Phys. Lett. 71 (1997) 96.

[9] S. Coffa et al., Appl. Phys. Lett. 73 (1998) 93.

[10] H. Przybylinska et al., Appl. Phys. Lett. 66 (1995) 490.

[11] M.S. Bresler et al., Appl. Phys. Lett. 67 (1995) 3599.

[12] J. Stimmer et al., Appl. Phys. Lett. 68 (1996) 3290.

[13] F.G. Anderson, Appl. Phys. Lett. 68 (1996) 2421.

[14] W. Fuhs et al., Phys. Rev. Lett. 61 (1988) 169.

[15] J.H. Chen et al., Appl. Phys. Lett. 67 (1995) 2182.

[16] S.J. Chang, D.K. Nayak and Y. Shiraki, J. Appl. Phys. 83 (1998) 1426.

[17] T.D. Culp et al., J. Appl. Phys. 83 (1998) 4918.

[18] J. Zavada and D. Zhang, Solid-State Electronics 38 (1995) 1285.

[19] S. Kim et al., Appl. Phys. Lett. 71 (1997) 231.

[20] M. Thaik et al., Appl. Phys. Lett. 71 (1997) 2641.

[21] M. Dalmer et al., Mater. Res. Soc. Symp. Proc. 482 (1998) 1021.

[22] D. Hansen et al., Appl. Phys. Lett. 72 (1998) 1244.

[23] A. Steckl et al., Appl. Phys. Lett. 73 (1998) 2450.

[24] A. Steckl and R. Birkhan, Appl. Phys. Lett. 73 (1998) 2450.

[25] M. Garter et al., Appl. Phys. Lett. 74 (1999) 182.

[26] H. Lozykowski, W. Jadwisienczak and I. Brown, Appl. Phys. Lett. 74 (1999) 1129.

[27] W. Choyke et al., Appl. Phys. Lett. 65 (1994) 1668.

[28] P. Baranov et al., Phys. Solid State 41 (1999) 32.

[29] R. Babunts et al., Phys. Solid State 42 (2000) 829.

[30] H. Lozykowski, W. Jadwisienczak and I. Brown, J. Appl. Phys. 88 (2000) 210.

[31] J. Gruber et al., J. Appl. Phys. 89 (2001) 7973.

[32] H. Lozykowski, W. Jadwisienczak and I. Brown, Solid State Commun. 110 (1999) 253.

[33] J. Gruber et al., J. Appl. Phys. 91 (2002) 2029.

[34] H. Heikenfeld et al., Appl. Phys. Lett. 75 (1999) 1189.

[35] H. Lozykowski et al., Appl. Phys. Lett. 77 (2000) 767. 
[36] H. Lozykowski, W. Jadwisienczak and I. Brown, Appl. Phys. Lett. 76 (2000) 861.

[37] H. Lozykowski, W. Jadwisienczak and I. Brown, Mat. Sci. Engin. B81 (2001) 140.

[38] J. Gruber et al., J. Appl. Phys. 92 (2002) 5127.

[39] J. Torvik et al., J. Appl. Phys. 81 (1997) 6343.

[40] J. Torvik et al., J. Appl. Phys. 82 (1997) 1824.

[41] A. Steckl et al., Appl. Phys. Lett. 75 (1999) 2184.

[42] D. Lee and A. Steckl, Appl. Phys. Lett. 82 (2003) 55.

[43] W. Jadwisienczak et al., J. Appl. Phys. 89 (2001) 4384.

[44] U. Vetter, J. Zenneck and H. Hofsäss, Intense ultra violet cathodoluminescence at $318 \mathrm{~nm}$ from $\mathrm{Gd}^{3+}$. doped AlN, accepted for publication in Appl. Phys. Lett.

[45] J. MacKenzi et al., Appl. Phys. Lett. 69 (1996) 2083.

[46] S.J. Pearton et al., Appl. Phys. Lett. 71 (1997) 1807.

[47] S.J. Pearton et al., J. Vac. Sci. Technol. A 16 (1998) 1627.

[48] K. Gurumurugan et al., Appl. Phys. Lett. 74 (1999) 3008.

[49] V. Dimitrova et al., Appl. Phys. Lett. 77 (2000) 478.

[50] U. Vetter et al., Mater. Res. Soc. Symp. Proc. 743 (2003).

[51] Q. Liu, F. Xu and T. Tanaka, Appl. Phys. Lett. 81 (2002) 3948.

[52] U. Vetter et al., Mater. Res. Soc. Symp. Proc. 744 (2003).

[53] U. Vetter et al., Diam. Relat. Mat. 12 (2003) 1182.

[54] Q. Liu et al., Appl. Phys. Lett. 81 (2002) 34.

[55] C. Ronda, T. Jüstel and H. Nikol, J. Alloys and Compounds 275-277 (1998) 669.

[56] J. Yum et al., J. Eletrochem. Soc. 150 (2003) H47.

[57] A. Steckl and J. Zavada, MRS Bulletin 24 (1999) 33.

[58] T. Gregorkiewicz and J. Langer, MRS Bulletin 24 (1999) 27.

[59] R. Moncorgé, L. Merkle and B. Zandi, MRS Bulletin 24 (1999) 21.

[60] W.X. Ni et al., Appl. Phys. Lett. 70 (1997) 3383.

[61] D.E. Wortman, C.A. Morrison and J.L. Bradshaw, J. Appl. Phys. 82 (1997) 2580.

[62] A. Terrasi et al., Appl. Phys. Lett. 70 (1997) 1712.

[63] E. Terukov et al., J. Non-Cryst. Solids 227-230 (1998) 488.

[64] G. Franzò et al., J. Appl. Phys 81 (1997) 2784.

[65] A.M. Emel'yanov, N.A. Sobolev and A.N. Yakimenko, Appl. Phys. Lett. 72 (1998) 1223.

[66] K. Takahei, R. Hogg and A. Taguchi, Mat. Res. Soc. Symp. Proc. 422 (1996) 267.

[67] M. Godlewski and K. Świa̧tek, J. Cryst. Growth 117 (1992) 634.

[68] M. Godlewski, K. Świątek and B. Monemar, J. Luminescence 58 (1994) 303.

[69] O. Gusev et al., Phys. Rev. B 64 (2001) 075302.

[70] Y. Jiaqi and S. Yongrong, J. Phys. C: Solid State Phys. 21 (1988) 3381.

[71] E. Bringuier, J. Appl. Phys. 70 (1991) 4505.

[72] K. Świa̧tek, A. Suchocki and B. Monemar, Appl. Phys. Lett. 56 (1990) 195. 
[73] T. Miyakawa and D. Dexter, Phys. Rev. B 1 (1970) 2961.

[74] F. Priolo et al., J. Appl. Phys. 78 (1995) 3874.

[75] J.D. Carey et al., Appl. Phys. Lett. 69 (1996) 3854.

[76] P. Baranov et al., Phys. Solid State 41 (1999) 783.

[77] N. Vinh et al., Phys. Rev. Lett. 90 (2003) 066401.

[78] J. Benton et al., J. Appl. Phys. 70 (1991) 2667.

[79] A. Cavallini et al., J. Appl. Phys. 85 (1999) 1582.

[80] V. Emtsev et al., Semiconductors 33 (1999) 1084.

[81] J. Evans-Freeman, P. Kan and N. Abdelgader, J. Appl. Phys. 92 (2002) 3755.

[82] V. Shmagin et al., Semiconductors 36 (2002) 178.

[83] G. Pasold et al., J. Appl. Phys. 93 (2003) 2289.

[84] R.L. Mössbauer, Z. Physik 151 (1958) 124.

[85] H. Frauenfelder and R. Steffen, Angular Correlations in Alpha, Beta, and Gamma-ray spectroscopy, edited by K. Siegbahn, North-Holland, 1965.

[86] M. Forker, Hyperfine Interact. 24-26 (1985) 907.

[87] G. Langouche, Hyp. Int. 45 (1989) 199.

[88] A. Bhagawat, K.G. Prasad and R.P. Sharma, Hyperfine Interactions 42 (1988) 977.

[89] F.S. Nasredinov et al., Phys. Stat. Sol. B 155 (1989) K113.

[90] V.F. Masterov et al., Appl. Phys. Lett. 72 (1998) 728.

[91] G. Stewart, Materials Forum 18 (1994) 177.

[92] G. Stewart, J. Magn. Magn. Mater. 118 (1993) 322.

[93] G. Stewart, S. Harker and D. Pooke, J. Phys.: Condens. Matter 10 (1998) 8269.

[94] M. Rams et al., J. Magn. Magn. Mater. 219 (2000) 15.

[95] J. Becquerel, Le Radium 4 (1907) 328.

[96] R. Tomaschek, Physik. Z. 33 (1932) 878.

[97] H. Van Vleck, J. Chem. Phys. 41 (1937) 67.

[98] B. Judd, Phys. Rev. 127 (1962) 750.

[99] G. Ofelt, J. Chem. Phys. 37 (1962) 511.

[100] C. Morrison and R. Leavitt, Handbook on the Physics and Chemistry of Rare Earths, edited by K.A. Gscheidner Jr. and L. Eyring, pp. 461-692, North-Holland Publishing Company, 1982.

[101] J. Jensen and A. Mackintosh, Rare Earth Magnetism: Structures and Excitation (Clarendon press, Oxford, 1991).

[102] A. Kaminskii, Laser CrystalsSpringer Series in Optical Sciences (Springer Verlag, Berlin, 1981).

[103] D. Newman and B. Ng, editors, Crystal Field Handbook (Cambridge University Press, 2000).

[104] B.R. Judd, Operator techniques in atomic spectroscopy (Princeton University Press (Princeton), 1997).

[105] S. Hüfner, Optical Spectra of Transparent Rare Earth Compounds (Academic Press, Inc. (London) Ltd, 1978).

[106] C. Görller-Walrand and K. Binnemans, Handbook on the Physics and Chemistry of Rare Earths, edited by K.A. Gscheidner Jr. and L. Eyring, volume 23, pp. 121-283, Elsevier Science B.V., 1996. 
[107] C. Görller-Walrand and K. Binnemans, Handbook on the Physics and Chemistry of Rare Earths, edited by K.A. Gscheidner Jr. and L. Eyring, volume 25, pp. 101-264, Elsevier Science B.V., 1998.

[108] A. Holleman and E. Wiberg, Lehrbuch der anorganischen Chemie, 81.-90. ed. (de Gruyter, 1976).

[109] I. Sobel'man, Introduction to the Theory of Atomic Spectra, First ed. (Pergamon Press Ltd, Oxford, 1972).

[110] K. Rajnak and B. Wybourne, Phys. Rev. 132 (1963) 280.

[111] B. Judd, Phys. Rev. 141 (1966) 4.

[112] B. Judd, H. Crosswhite and H. Crosswhite, Phys. Rev. 169 (1968) 130.

[113] H. Marvin, Phys. Rev. 71 (1947) 102.

[114] Z. Goldschmidt, A. Pasternak and Z. Goldschmidt, Phys. Lett. A 28 (1968) 265.

[115] G. Racah, Phys. Rev. 61 (1942) 186.

[116] G. Racah, Phys. Rev. 62 (1942) 438.

[117] G. Racah, Phys. Rev. 63 (1943) 438.

[118] G. Racah, Phys. Rev. 76 (1949) 1352.

[119] G. Dieke, Spectra and Energy Levels of Rare Earth Ions in Crystals (Wiley-Interscience, New York, 1968).

[120] D. Garcia and M. Faucher, Handbook on the Physics and Chemistry of Rare Earths, edited by K.A. Gscheidner Jr. and L. Eyring, pp. 263-304, Elsevier Science B.V., 1995.

[121] S.Y. Rena and J.D. Dow, J. Appl. Phys. 81 (1997) 1877.

[122] M. Klintenberg, Rare-Earth Polarized Absorption Spectra as a Structural Tool, PhD thesis, University of Uppsala, 1997.

[123] J. Axe Jr., J. Chem. Phys. 39 (1963) 1154.

[124] K. Adler and R. Steffen, The Electromagnetic Interaction in Nuclear Spectroscopy, edited by W. Hamilton, pp. 1-38, North-Holland, 1975.

[125] P. Yu and M. Cardona, Fundamentals of Semiconductors (Springer, 1996).

[126] Y. Goldberg, In Levinshtein et al. [134].

[127] G. Yu et al., Appl. Phys. Lett. 70 (1997) 3209.

[128] V. Bourgrov et al., In Levinshtein et al. [134].

[129] D. Dexter, Phys. Rev. 108 (1957) 630.

[130] L. Riseberg and H. Moos, Phys. Rev. B 174 (1968) 429.

[131] C. Struck and W. Fonger, Nucl. Instrum. Meth. B 10 (1975) 1.

[132] A. Ellens et al., Phys. Rev. B 55 (1997) 173.

[133] A. Ellens et al., Phys. Rev. B 55 (1997) 180.

[134] M. Levinshtein, S. Rumyantsev and M. Shur, editors, Properties of Advanced Semiconductor Materials (John Wiley \& Sons, 2001).

[135] H. Heuser, Gewöhnliche Differentialgleichungen (Teubner, Stuttgart, 1989).

[136] H. Lozykowski, Phys. Rev. B 48 (1993) 17758.

[137] G. Franzo, V. Vinciguerra and F. Priolo, Appl. Phys. A 69 (1999) 3.

[138] T. Förster, Ann. Physik 6 (1948) 55.

[139] D. Dexter, J. Chem. Phys. 21 (1953) 836.

[140] M. Inokuti and F. Hirayama, J. Chem. Phys. 43 (1965) 1978.

[141] J. Hegarty, D. Huber and W. Yen, Phys. Rev. B 25 (1982) 5638. 
[142] M.E. Kompan and I.Y. Shabanov, Phys. Solid State 39 (1997) 1030.

[143] F. Priolo et al., Phys. Rev. B 57 (1998) 4443.

[144] M. Reid, F-shell empirical programs and examples, 1988.

[145] M. Reid, Transition intensities, In Newman and Ng [103], chap. 10, pp. 190-226.

[146] G. Liu, Lanthanide and actinide optical spectra, In Newman and Ng [103], chap. 4, pp. 65-82.

[147] C. Nielson and G. Koster, Spectroscopy coefficients for the $\mathrm{p}^{n}, \mathrm{~d}^{n}$ and $\mathrm{f}^{n}$ configurations (MIT Press, 1963).

[148] R. Storn and K. Price, J. Glob. Optim. 11 (1997) 314.

[149] U. Pennig and U. Vetter, Differential Evolution in the Spektralanalyse, Universität Göttingen, unpublished, 2003.

[150] M. Nogami and T. Hayakawa, Phys. Rev. B 56 (1997) 56.

[151] F. Suyver and A. Meijerink, Chemisch 2 Weekblad 4 (2002) 12.

[152] S. Tanabe and T. Hanada, J. Appl. Phys. 76 (1994) 3730.

[153] J. Chwalek and G. Paz-Pujalt, Appl. Phys. Lett. 66 (1995) 410.

[154] R. Paschotta et al., J. Opt. Soc. Am. B 14 (1997) 1213.

[155] P. dos Santos et al., J. Appl. Phys. 90 (2001) 6550.

[156] J. Qiu and Y. Kawamoto, J. Appl. Phys. 91 (2002) 954.

[157] N. Rakov et al., J. Appl. Phys. 92 (2002) 6337.

[158] G. Dominiak-Dzik et al., J. Phys.: Condens. Matter 12 (2000) 5495.

[159] L.D. Merkle et al., J. Appl. Phys. 72 (1992) 4269.

[160] J. Gruber et al., Phys. Rev. B 40 (1989) 9464.

[161] S. Lakshman and C. Jayasankar, J. Phys. C: Solid State Phys. 17 (1984) 2967.

[162] R. Wegh et al., Phys. Rev. B 56 (1997) 13841.

[163] B. Liu et al., J. Luminescence 101 (2003) 155.

[164] J. Sytsma, G. Imbusch and G. Blasse, J. Chem. Phys. 91 (1983) 1456.

[165] R.Y. Abdulsabirov et al., J. Luminescence 94-95 (2001) 113.

[166] M. ter Heerdt et al., J. Luminescence 100 (2002) 115.

[167] D.G. Gao et al., Solid State Comm. 121 (2002) 145.

[168] H. Hofsäss, Hyperfine Interact. 97/98 (1996) 247.

[169] P.A. Doyle and P.S. Turner, Acta Cryst. A 24 (1968) 390.

[170] H.C. Hofsäss, Channeling von Elektronen und Positronen zur Lokalisierung von radioaktiven Sondenatomen in Metallen und Halbleitern, Dissertation, Universit"at Konstanz, 1987.

[171] J.P. Schiffer, P.N. Parks and J. Heberele, Phys. Rev. 133 (1964) 1553.

[172] J. Andersen, K. Eriksen and E. Lægsgaard, Phys. Scripta 24 (1981) 588.

[173] J. Andersen et al., Phys. Scripta 28 (1983) 308.

[174] P. Lervig, J. Lindhard and V. Nielsen, Nuclear Physics A96 (1967) 481.

[175] G. Lindner, Kombination von Elektron-Channeling- und Hyperfein-Wechselwirkungs-Messungen mit radioaktiven In-Sonden in Kupfer, Dissertation, Universität Konstanz, 1984.

[176] J.W. Mayer, L. Eriksson and J.A. Davies, Ion implantation in semiconductors (Academic Press, 1970).

[177] U. Wahl, Hyperfine Interact. 129 (2000) 349. 
[178] U. Vetter et al., Hyperfine Interact. 136/137 (2002) 607.

[179] H. Bialas and H. Stolz, Z. Phys. B 21 (1975) 319.

[180] K. Bharuth-Ram et al., Nucl. Instrum. Meth. B 190 (2002) 835.

[181] U. Wahl et al., J. Appl. Phys. 88 (2000) 1319.

[182] B. De Vries et al., Phys. Stat. Sol. (c) 0 (2002) 453.

[183] U. Wahl et al., Nucl. Instrum. Meth. B 175-177 (2001) 262.

[184] J. Araújo et al., Nucl. Instrum. Meth. B 191 (2002) 317.

[185] U. Wahl, A. Vantomme and G. Langouche, Nucl. Instrum. Meth. B 148 (1999) 492.

[186] U. Wahl et al., Phys. Rev. Lett. 79 (1997) 2069.

[187] U. Vetter et al., Lattice location studies of rare earth impurities in 3C-, 4H- and 6H-SiC, accepted for publication in diamond and thin films.

[188] R. Firestone, Table of Isotopesvolume I-II, 8. ed. (John Wiley \& Sons, Inc., 1996).

[189] E. Browne, J. Dairiki and R. Doebler, Table of Isotopes, 7. ed. (John Wiley \& Sons, Inc., 1978).

[190] J. Cabrera et al., Nucl. Instr. Meth. A 312 (1992) 364.

[191] J.F. Ziegler, J.P. Biersack and U. Littmark, The stopping and range of ions in solids (Pergamon Press, New York, 1999).

[192] L. Palmetshofer, M. Gritsch and G. Hobler, Mat. Sci. Engin. B 81 (2001) 83.

[193] K. Mielenz, Optical radiation measurementsvolume 3 (Academic Press, 1986).

[194] L. Ozawa, Cathodoluminescence - Theory and Applications (VCH, 1990).

[195] Y. Wang and A. Steckl, Appl. Phys. Lett. 82 (2003) 502.

[196] P. Hovington, D. Drouin and R. Gauvin, Scanning 19 (1997) 1.

[197] D. Drouin, P. Hovington and R. Gauvin, Scanning 19 (1997) 20.

[198] P. Hovington et al., Scanning 19 (1997) 29.

[199] N. Greenwood and T. Gibb, Mössbauer Spectroscopy (Chapman and Hall Ltd., London, 1971).

[200] G. Shenoy et al., Mössbauer Spectroscopy and its Chemical Applications, edited by J. Stevens and G. Shenoy, pp. 117-123, American Chem. Soc., 1991.

[201] C. Sansonetti, M. Salit and J. Reader, Applied Optics 35 (1996) 74.

[202] E. Kowalski, Nuclear Electronics (Springer Verlag, Berlin, 1970).

[203] M. Smith et al., Appl. Phys. Lett. 67 (1995) 3387.

[204] G. Chen et al., Appl. Phys. Lett. 68 (1996) 2784.

[205] J. Im et al., Appl. Phys. Lett. 70 (1997) 631.

[206] L. Costa, K. Mielenz and F. Grum, Correction of emission spectra, [193], chap. 4, pp. 139-173.

[207] W. Forsythe and E. Adams, J. Opt. Soc. Am. 35 (1945) 108.

[208] B. Edlén, Metrologia 2 (1966) 71.

[209] G. Bönsch and E. Potulski, Metrologia 35 (1998) 133.

[210] M. Dalmer, Emissionschanneling-Untersuchungen an ionenimplantierten Gruppe III-Nitriden mit hexagonaler Gitterstruktur, Dissertation, Universität Konstanz, 1999.

[211] H. Hofsäss et al., Nucl. Instrum. Meth. B 63 (1992) 83.

[212] H. Hofsäss, U. Wahl and S.G. Jahn, Hyperfine Interact. 84 (1994) 27. 
[213] U. Wahl et al., Nucl. Instrum. Meth. B 136-138 (1998) 744.

[214] H. Hofsäss et al., Electron emission channeling spectroscopy using X-ray CCD detectors, nucl. instrum. meth. a, accepted for publication.

[215] Deetee, high performance diodes, XRA-series, Product specification sheet, available through http://www.deetee.com, 2000.

[216] Deetee, high performance diodes, XRB-series, Product specification sheet, available through http://www.deetee.com, 2000.

[217] EG\&G ORTEC, Models 142A,142B and 142C Preampflifiers Operating and Service Manual.

[218] G. Weyer, Nucl. Instrum. Meth. 186 (1981) 201.

[219] J. Gancedo, M. Garcia and J. Marco, Hyperfine Interact. 66 (1991) 83.

[220] P. Schaaf et al., Nucl. Instrum. Meth. B 53 (1991) 184.

[221] E. Kugler, Hyperfine Interact. 129 (2000) 23.

[222] H. Hofsäss et al., Diam. Relat. Mater. 3 (1994) 137.

[223] H. Hofsäss and C. Ronning, Proc. 2nd Int. Conf. on Beam Processing of Advanced Materials, edited by J. Singh, S.M. Copley and J. Mazumder, p. 29, ASM Int., Materials Park, 1996.

[224] M. Uhrmacher et al., Nucl. Instrum. Meth. B 9 (1985) 234.

[225] M. Uhrmacher et al., Nucl. Instrum. Meth. B 139 (1998) 306.

[226] L. van Pieterson et al., Phys. Rev. B 65 (2002) 045113.

[227] X. Wu et al., J. Luminescence 72-74 (1997) 284.

[228] F. Lu et al., J. Appl. Phys. 92 (2002) 2457.

[229] C. Ronning et al., J. Appl. Phys. 87 (2000) 2149.

[230] J. Hong et al., Solid-State Electronics 41 (1997) 681.

[231] C. Zetterling et al., J. Appl. Phys. 82 (1997) 2990.

[232] X. Tang et al., Appl. Phys. Lett. 72 (1998) 1501.

[233] Y. Lan et al., J. Crystal Growth 207 (1999) 247.

[234] K. Kornitzer et al., J. Crystal Growth 201/202 (1999) 441.

[235] S. Tungasmita et al., Materials Science Forum 338-342 (2000) 1519.

[236] Y. Shishkin et al., Phys. Stat. Sol. (a) 188 (2001) 591.

[237] J. Li et al., Appl. Phys. Lett. 81 (2002) 3365.

[238] J. Harris, R. Youngman and R. Teller, J. Mater. Res. 5 (1990) 1763.

[239] S. Schweizer et al., Phys. Stat. Sol. (b) 219 (2000) 171.

[240] V. Davidov et al., Phys. Rev. B 58 (1998) 12899.

[241] J. Nipko and C. Loong, Phys. Rev. B 57 (1998) 10550.

[242] L. Bergman et al., Phys. Rev. B 59 (1999) 12977.

[243] L. Liu et al., J. Appl. Phys. 92 (2002) 5183.

[244] K. Miwa and A. Fukumoto, Phys. Rev. B 48 (1993) 7897.

[245] S. Strite and H. Morkoç, J. Vac. Sci. Technol. B 19 (1992) 1237.

[246] H. Morkoç et al., J. Appl. Phys. 76 (1994) 1363.

[247] O. Ambacher, J. Phys. D: Appl. Phys. 31 (1998) 2653. 
[248] T. Weeks Jr. et al., J. Mater. Res. 11 (1996) 1011.

[249] U. Wahl, U. Vetter and M. Dietrich, Emission channeling data recorded for $\beta^{-}$and conversion electrons appearing in the decay ${ }^{147} \mathrm{Nd} \rightarrow{ }^{147} \mathrm{Pm}$, after implantations into AlN at ISOLDE. Mai 2003, unpublished.

[250] J. Gruber et al., J. Appl. Phys. 81 (1997) 6585.

[251] S. Tanabe et al., Phys. Rev. B 53 (1996) 8358.

[252] T. Taniguchia, M. Akaishi and S. Yamaoka, J. Mater. Res. 14 (1999) 162.

[253] T. Taniguchia and S. Yamaoka, J. Crystal Growth 222 (2001) 549.

[254] W. Zhang, Appl. Phys. Lett. 81 (2001) 3356.

[255] O. Mishima et al., Appl. Phys. Lett. 53 (1988) 962.

[256] T. Taniguchi et al., Appl. Phys. Lett. 81 (2002) 4145.

[257] H. Feldermann, Nucleation and growth of ion-beam-deposited cubic boron nitride thin films, Dissertation, Universität Göttingen, 2002.

[258] P. Weilhammer et al., Nucl. Instrum. Meth. B 384 (1996) 159.

[259] V. Shipilo et al., Phys. Status Solidi A 108 (1988) 431.

[260] K. Karch and F. Bechstedt, Phys. Rev. B 56 (1997) 7404.

[261] S. Davidov, Semiconductors 36 (2002) 45.

[262] R. Davis, Physica B 185 (1993) 1.

[263] Y. Park, SiC Materials and Devices, ed. Y.S. Park, Semiconductors and Semimetals, edited by R. Williardson and E. Weber, Adademic Press, 1998.

[264] A. Lebedev and V. Chelnokov, Semiconductors 33 (1999) 999.

[265] R. Devaty and W. Choyke, Phys. Stat. Sol. (a) 162 (1997) 5.

[266] G. Pensl and W. Choyke, Physica B 185 (1993) 264.

[267] J. Meier et al., Physica B 185 (1993) 207.

[268] N. Achtziger et al., Nucl. Instrum. Meth. B 136-138 (1998) 756.

[269] E. Wendler, A. Heft and W. Wesch, Nucl. Instrum. Meth. B 141 (1998) 105.

[270] A. Hallén et al., Nucl. Instrum. Meth. B 186 (2002) 186.

[271] A. Kozanecki et al., Nucl. Instrum. Meth. B 148 (1999) 512.

[272] A. Zywietz, K. Karch and F. Bechstedt, Phys. Rev. B 54 (1996) 1791.

[273] G. Braunstein and R. Kalish, Appl. Phys. Lett. 38 (1981) 416.

[274] J.F. Prins, Diam. Relat. Mater. 4 (1995) 580.

[275] D. Saada, J. Adler and R. Kalish, Phys. Rev. B 59 (1999) 6650.

[276] A. Burchard et al., Physica B 195 (1993) 150.

[277] H. Hofsäss and G. Lindner, Phys. Rep. 201 (1991) 121.

[278] H. Hofsäss et al., J. Appl. Phys. 81 (1999) 2566.

[279] M. Restle et al., Appl. Phys. Lett. 66 (1995) 2733.

[280] E. Storbeck et al., Nucl. Instrum. Meth. B 85 (1994) 503.

[281] G. Malashkevich et al., JETP Letters 77 (2003) 291.

[282] C. Manfredotti et al., Nucl. Instrum. Meth. A 410 (1998) 96.

[283] S. Han et al., Diam. Relat. Mater. 9 (2000) 1008. 
[284] A. Galbiati et al., Nucl. Instrum. Meth. A 466 (2001) 52.

[285] P. Bergonzo et al., Phys. Status Solidi A 185 (2001) 167.

[286] H. Hofsäss et al., Nucl. Instrum. Meth. B 80/81 (1993) 176.

[287] H. Sternschulte, Lumineszenzuntersuchungen an dotiertem und undotiertem Diamant, Dissertation, Universität Ulm, 1999.

[288] M. Gasnier, Handbook on the Physics and Chemistry of Rare Earths, edited by K.A. Gscheidner Jr. and L. Eyring, volume 20, pp. 105-206, Elsevier Science B.V., 1995.

[289] V. Puzikov, A. Semenov and O. Tovmachenko, J. Superhard Materials 22 (2000) 49.

[290] H. Savaloni, M. Playera and G. Marr, Nanotechnology 1 (1990) 145.

[291] E. Gu et al., J. Phys. Chem. Sol. 53 (1992) 127.

[292] R. Meltzer et al., Phys. Rev. B 60 (1999) 14012.

[293] R. Meltzer and K. Hong, Phys. Rev. B 61 (2000) 3396.

[294] Y. Kanemitsu, Y. Fukunishi and T. Kushida, Appl. Phys. Lett. 77 (2000) 211.

[295] R. Meltzer et al., J. Luminescence 94-95 (2001) 221.

[296] X. Zhao et al., Int. J. Mod. Phys. B 16 (2002) 4294.

[297] C. Rozo et al., Mat. Res. Soc. Symp. Proc. 737 (2003).

[298] I. Gerhards et al., Surf. Coat. Technol. 158 (2002) 114.

[299] A. Burnham and G. Jameson, J. Vac. Sci. Technol. A, Vac. Surf. Films 5 (1987) 1713.

[300] J. Gruber et al., J. Appl. Phys. 73 (1993) 1929.

[301] G. Koster, Solid State Phys. 5 (1957) 172.

[302] W. Xu et al., J. Appl. Phys. 75 (1994) 4180.

[303] S. Kishimoto and K. Hirao, J. Appl. Phys. 80 (1996) 1965.

[304] N. Antoine-Vincent et al., J. Appl. Phys. 93 (2003) 5222.

[305] D. Thomas and J. Hopfield, Phys. Rev. 150 (1968) 680.

[306] G. Wellenhofer et al., Phys. Rev. B 53 (1996) 6071. 



\section{Acknowledgments}

Bei meinem Doktorvater, Herrn Prof. Dr. Hans Hofsäß, möchte ich mich an dieser Stelle ganz besonders für die unvergleichliche Unterstützung und das in mich gesetzte Vertrauen bei allen Forschungsvorhaben bedanken. Ihm habe ich es zu verdanken, dass ich letzten Endes doch weiter der Physik treu bleiben konnte.

Herrn Prof. Dr. Rainer G. Ulbrich danke ich für die spontane Bereitschaft, diese Arbeit zu begutachten.

Sincere thanks is extended to Dr. Michael F. Reid, University of Canterbury, New Zealand, for answering endless questions about numerical details and the optical properties of the lanthanides. I am also indebted to him for the permission to use his algorithms for lanthanide absorption spectroscopy.

Prof. Dr. John B. Gruber, University of San José, California, USA, is gratefully acknowledged for the fruitful collaboration and his help in performing crystal field calculations and assignments of experimental data.

Bei Herrn Dr. Marc Dietrich möchte ich mich herzlichst für die Hilfe bei den Implantationen an ISOLDE bedanken. Erst sein überdurchschnittliches Engagement hat es ermöglicht in so kurzer Zeit eine grosse Anzahl an Emissionschanneling- und Mössbauermessungen durchzuführen.

Dr. Takachi Taniguchi, National Institute for Materials Science, Ibaraki, Japan, is given sincere thanks for supplying and annealing the cBN samples.

Prof. Dr. Krish Bharuth-Ram is acknowledged for supplying the diamond samples and for his help concerning the CEMS detector.

Einen großen Anteil am Gelingen dieser Arbeit tragen die Werkstätten des 2. Physikalischen Institutes. Erst durch die schnelle und kompetente Umsetzung wirrer Bleistiftzeichnungen in reale dreidimensionale Objekte konnten Fortschritte bei Forschungsvorhaben erzielt werden. Stellvertretend für die Mechanikwerkstatt sei mein Dank an den Werkstattleiter, Herrn Klaus Arndt, sowie seinen Stellvertreter, Herrn Christoph Schmidt, gerichtet. Auf Seiten der Elektronikwerkstatt möchte ich mich insbesondere bei Herrn Volker Ebrecht für unermüdliche Neuentwicklungen im Detektorbereich und beim Werkstattleiter, Herrn Kurt Schemmerling für wiederholtes Neuentwickeln der Pulseinheit bedanken. Ebenso geht grosser Dank an Herrn Herwig Schultz für Hilfe in vielen Angelegenheiten.

Ganz besonders herzlich möchte ich mich auch bei Frau Wohlfarth bedanken für ihre unermüdliche Unterstützung bei allen Verwaltungsangelegenheiten und für die tolle Atmosphäre am Institut, zu der sie wesentlich beiträgt.

Des weiteren gilt grosser Dank all denen, die irgendwie in das Zustandekommen dieser Arbeit, freiwillig oder unfreiwillig, verwickelt waren:

- Herrn Dr. Ulrich Wahl gebührt mein Dank für die Hilfe in allen das Emissionchanneling betreffenden Fragen. 
- Sören Eyhusen, der mir ein sehr sympathischer Begleiter in all der Zeit war.

- Daniel Schwen für die Unterstützung mit Schoka-Cola und Musik, und für seinen Beitrag zu: "C - weit weg vom Ansi-Standard".

- PD Dr. Petra Reinke für die vielen Anregungen, die Kompetenz in physikalischen Fragen, und das gelegentlich dringend nötige Aufmuntern.

- Herrn Dr. Lüder Ziegeler für das unermüdliche und geduldige Auspacken und Überprüfen der unzähligen radioaktiven Proben aus Genf. Von ihm konnte ich viel über den veantwortungsvollen Umgang mit Radioaktivät lernen.

- Herr PD Dr. Carsten Ronning für wertvolle Hinweise beim Erstellen dieser Arbeit, wichtige Tips in Sachen ADONIS, sowie das lange lustige Nebeneinander im selben Büro.

- Dr. Michael Uhrmacher für die Unterstützung bei Implantationen sowie manch anderem.

- Helge Kröger und Lucie Hamdi für das genaue Durchlesen dieser Arbeit.

- Der Rest der Hossaff-Group: Inga Gerhards, Stefano Virdis, Dr. Naigui Shang, Jan Zenneck und Holger Stillrich, für die super Stimmung während der ganzen Zeit.

- Prof. Dr. Peter Schaaf für all die Hilfe in Sachen Mössbauerspektroskopie.

- Ulrich Pennig für unermüdliches gemeinsames Programmieren..

Mein tief empfundener Dank geht an dieser Stelle an meine Eltern, die mich nicht nur zu Studien-, sondern auch Promotionszeiten in jederlei Hinsicht unterstützt haben.

Meine ganze Liebe gilt meiner Frau Olga und unserem Sohn Nikita, für die Unterstützung und das Verständnis während der letzten drei Jahre, in denen ich all zu oft nächtelang und am Wochenende im Labor verschwunden war. 


\section{Curriculum vitae}

Persönliche Daten:

Name: $\quad$ Ulrich Vetter

Geburtstag: $\quad$ 8. Juni 1971

Geburtsort: $\quad$ Stuttgart-Bad Canstatt, Deutschland

Nationalität: deutsch

Familienstand: $\quad$ verheiratet, ein Kind

Bildungsweg:

\begin{tabular}{|c|c|}
\hline $1977-1981$ & Grund-und Hauptschule Hemmingen \\
\hline $1981-1990$ & Gymnasium Korntal-Münchingen, Abitur 1990 \\
\hline 1990-1994 & Universität Konstanz, Vordiplom 1993 \\
\hline 1994-1995 & Fudan-Universität, Shanghai, PR China \\
\hline \multirow{3}{*}{ 1995-1998 } & Studium der chinesischen Sprache \\
\hline & Universität Konstanz \\
\hline & Diplom in Physik \\
\hline
\end{tabular}

Arbeitserfahrungen:

1992-1998 AEG/Siemens Electrocom Konstanz

mehrere 18-monatige Tätigkeiten als Werkstudent

1998-1999 Siemens Electrocom Konstanz

Vollzeittätigkeit in der Softwareentwicklung

1999-2000 Fraunhofer Institut für Arbeitswissenschaften

und Technologiemanagement (IAT), Stuttgart

wissenschaftlicher Angestellter

2000-2003 2. Physikalisches Institut, Universität Göttingen

wissenschaftlicher Angestellter 
